\title{
Dehydrogenative Coupling of Terminal Alkynes with O/N-Based Monohydrosilanes Catalyzed by Rare-Earth Metal Complexes
}

Xiancui Zhu, ${ }^{*} \uparrow$ Dianjun Guo, ${ }^{\dagger}$ Zeming, Huang,, Tian Sheng, ${ }^{*}{ }^{\dagger}$ Shaowu Wang, ${ }^{*} \uparrow$, 屯, ${ }^{\S}$ Mengke Pan, ${ }^{\dagger}$ Ling Zha, ${ }^{\dagger}$ Shuangliu, Zhou ${ }^{\dagger}$

$\dagger$ Key Laboratory of Functional Molecular Solids, Ministry of Education, Anhui Laboratory of Molecule-Based Materials, College of Chemistry and Materials Science, Anhui Normal University, Wuhu, Anhui 241002 (P. R. China)

* Anhui Laboratory of Clean Catalytic Engineering, Anhui Laboratory of Functional Complexes for Materials Chemistry and Application, College of Biological and Chemical Engineering, Anhui Polytechnic University, Wuhu, Anhui 241000 (P. R. China)

$\S$ State Key Laboratory of Organometallic Chemistry, Shanghai Institute of Organic Chemistry, Chinese Academy of Sciences, Shanghai 200032 (P. R. China) 


\section{Table of contents}

1. Molecular structures of complexes 1 b-c........................................... 1

Figure S1. Molecular structure of complex 1b.................................................. 1

Figure S2. Molecular structure of complex 1c..................................................... 1

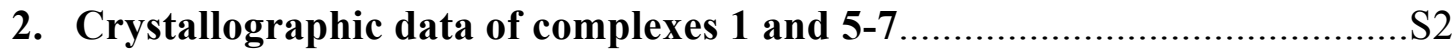

Table S1. Crystallographic data of complexes 1 a-c......................S2

Table S2. Crystallographic data of complexes $5-7 \ldots \ldots \ldots \ldots \ldots \ldots \ldots . . . \ldots . \ldots . \ldots$

3. Kinetic Isotope Effect Experiment.................................. 4

4. Compound data and ${ }^{1} \mathrm{H}$ and ${ }^{13} \mathrm{C}$ NMR spectra......................... 5

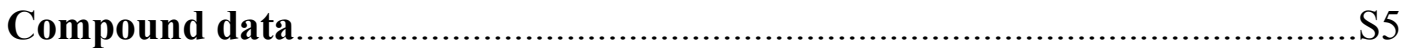

Figure S3. ${ }^{1} \mathrm{H}$ NMR spectrum of complex 1a................................ 12

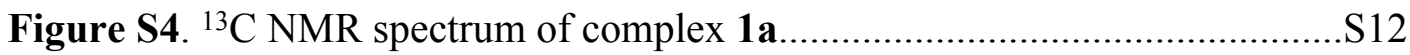

Figure S5. ${ }^{1} \mathrm{H}$ NMR spectrum of complex 5a................................ 13

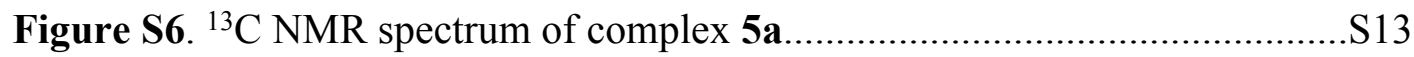

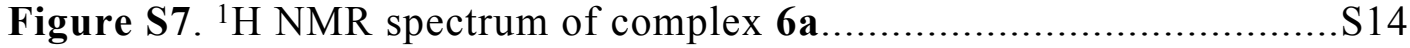

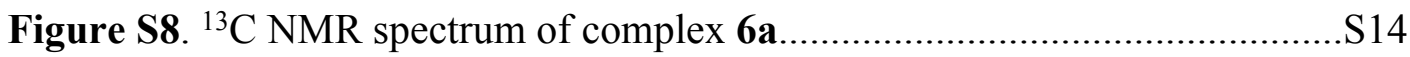

Figure S9. ${ }^{1} \mathrm{H}$ NMR monitoring the catalytic reaction by $\mathbf{5 a}$ a.....................S15

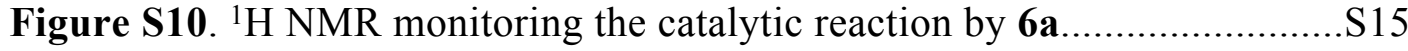

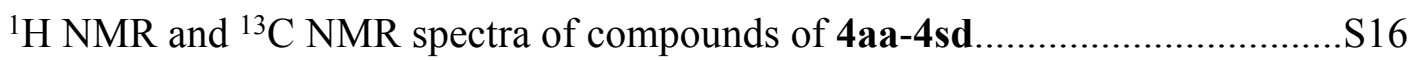

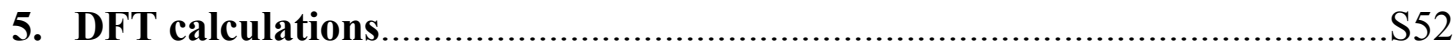

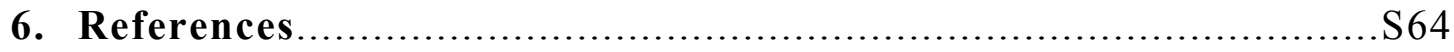


Molecular structure of complex $1 \mathrm{~b}$

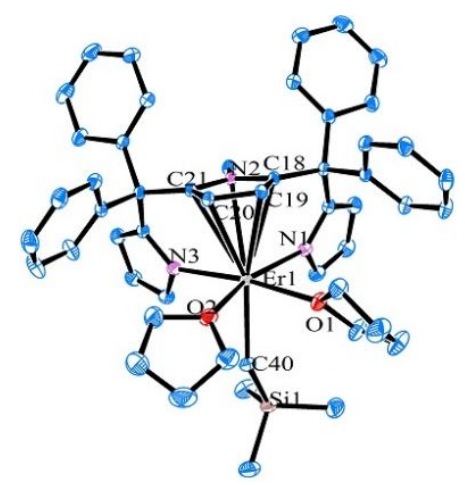

Figure S1. Molecular structure of complex 1b with ellipsoids at $30 \%$ probability level. Hydrogen atoms were omitted for clarity. Selected bond lengths $(\AA)$ : Er1-N1 2.311(2), Er1-N2 2.8520(18), Er1-N3 2.3642(18), Er1-C18 2.868(2), Er1-C19 2.844(2), Er1-C20 2.853(2), Er1-C21 2.884(2), Er1-C40 2.413(2), Er1-O1 2.4641(17), Er1-O2 2.4866(18).

Molecular structure of complex 1c

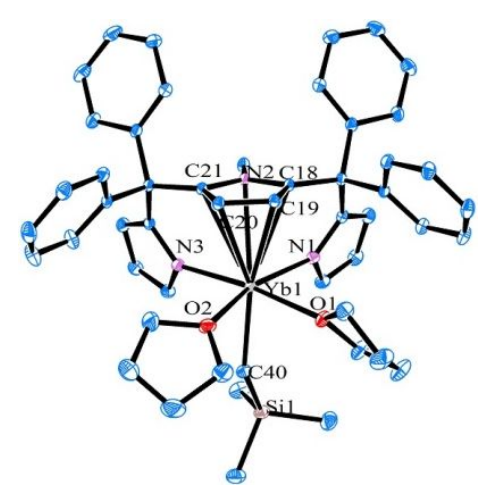

Figure S2. Molecular structure of complex 1c with ellipsoids at $30 \%$ probability level. Hydrogen atoms were omitted for clarity. Selected bond lengths $(\AA)$ : Yb1-N1 2.291(3), Yb1-N2 2.864(2), Yb1-N3 2.342(3), Yb1-C18 2.870(3), Yb1-C19 2.839(3), Yb1-C20 2.846(3), Yb1-C21 2.889(3), Yb1-C40 2.392(3), Yb1-O1 2.441(2), Yb1-O2 2.464(2). 
Table S1. Crystallographic data for complexes 1a-c.

\begin{tabular}{|c|c|c|c|}
\hline & $1 \mathbf{a}$ & $\mathbf{1 b}$ & 1c \\
\hline Formula & $\mathrm{C}_{51} \mathrm{H}_{58} \mathrm{~N}_{3} \mathrm{O}_{2} \mathrm{SiY}$ & $\mathrm{C}_{51} \mathrm{H}_{58} \mathrm{~N}_{3} \mathrm{O}_{2} \mathrm{SiEr}$ & $\mathrm{C}_{51} \mathrm{H}_{58} \mathrm{~N}_{3} \mathrm{O}_{2} \mathrm{SiYb}$ \\
\hline FW & 862.00 & 940.35 & 946.13 \\
\hline Space group & $C_{1} / c$ & $P \overline{1}$ & $P \overline{1}$ \\
\hline $\mathrm{T}(\mathrm{K})$ & 293(2) & $293(2)$ & $293(2)$ \\
\hline Crystal system & Monoclinic & Triclinic & Triclinic \\
\hline $\mathrm{a}(\AA)$ & $27.692(2)$ & $10.0210(8)$ & $10.0319(11)$ \\
\hline $\mathrm{b}(\AA)$ & $12.3946(9)$ & $12.7342(11)$ & 12.7071(11) \\
\hline c $(\AA)$ & 17.2381(13) & 18.6799(16) & 18.6626(18) \\
\hline$\alpha(\operatorname{deg})$ & 90 & $78.8020(10)$ & $78.6850(10)$ \\
\hline$\beta(\operatorname{deg})$ & $121.1660(10)$ & $84.6660(10)$ & $84.7900(10)$ \\
\hline$\gamma(\operatorname{deg})$ & 90 & $69.8040(10)$ & $69.8530(10)$ \\
\hline $\mathrm{Z}$ & 4 & 2 & 2 \\
\hline $\mathrm{V}\left(\AA^{3}\right)$ & $5062.7(6)$ & 2193.7(3) & 2189.4(4) \\
\hline $\mathrm{D}_{\mathrm{c}}\left(\mathrm{Mgm}^{-3}\right)$ & 1.131 & 1.424 & 1.435 \\
\hline$\mu\left(\mathrm{mm}^{-1}\right)$ & 1.213 & 1.984 & 2.207 \\
\hline $\mathrm{F}(000)$ & 1816 & 966 & 970 \\
\hline Reflns collected & 21477 & 25198 & 19972 \\
\hline Unique reflns & 10700 & 9831 & 7598 \\
\hline Parameters & 568 & 527 & 527 \\
\hline Goodness of fit & 0.972 & 1.038 & 1.020 \\
\hline$\theta$ range (deg) & 1.719 to 27.517 & 1.112 to 27.557 & 1.113 to 25.000 \\
\hline $\mathrm{R}_{1}(I>2 \sigma(I))$ & 0.0605 & 0.0236 & 0.0317 \\
\hline $\mathrm{wR}_{2}(I>2 \sigma(I))$ & 0.1500 & 0.0605 & 0.0671 \\
\hline $\mathrm{R}$ (int) & 0.0924 & 0.0232 & 0.0197 \\
\hline \multirow{2}{*}{ Largest diff. peak and hole (e. $\left.\AA^{-3}\right)$} & 0.792 & 0.846 & 0.569 \\
\hline & -0.467 & -0.423 & -0.659 \\
\hline
\end{tabular}


Table S2. Crystallographic data for complexes 5-7c.

\begin{tabular}{|c|c|c|c|c|}
\hline & $5 \mathbf{a}$ & $5 c$ & 6c & $7 \mathrm{c}$ \\
\hline Formula & $\mathrm{C}_{98} \mathrm{H}_{80} \mathrm{~N}_{6} \mathrm{OY}_{2}$ & $\mathrm{C}_{94} \mathrm{H}_{72} \mathrm{~N}_{6} \mathrm{Yb}_{2}$ & $\mathrm{C}_{82} \mathrm{H}_{72} \mathrm{~N}_{6} \mathrm{OYb}_{2}$ & $\mathrm{C}_{82} \mathrm{H}_{72} \mathrm{~N}_{6} \mathrm{O}_{2} \mathrm{Yb}_{2}$ \\
\hline FW & 1535.50 & 1631.65 & 1503.53 & 1519.53 \\
\hline Space group & $P \overline{1}$ & $P \overline{1}$ & $P \overline{1}$ & $C_{1} / c$ \\
\hline $\mathrm{T}(\mathrm{K})$ & $293(2)$ & $293(2)$ & $293(2)$ & $293(2)$ \\
\hline Crystal system & Triclinic & Triclinic & Triclinic & Monoclinic \\
\hline $\mathrm{a}(\AA)$ & $13.3315(6)$ & $12.6758(4)$ & $13.2061(5)$ & $24.5019(13)$ \\
\hline $\mathrm{b}(\AA)$ & $13.4482(6)$ & $13.2109(4)$ & $14.6544(5)$ & $22.3632(13)$ \\
\hline c $(\AA)$ & $24.7108(12)$ & $14.6850(4)$ & $22.2507(9)$ & $18.2521(14)$ \\
\hline$\alpha(\operatorname{deg})$ & $90.151(2)$ & $109.9350(10)$ & $78.388(2)$ & 90 \\
\hline$\beta(\mathrm{deg})$ & $96.064(2)$ & $103.3260(10)$ & $73.235(2)$ & $128.4989(9)$ \\
\hline$\gamma(\operatorname{deg})$ & $94.660(2)$ & $100.048(2)$ & $79.160(2)$ & 90 \\
\hline Z & 2 & 1 & 2 & 4 \\
\hline $\mathrm{V}\left(\AA^{3}\right)$ & $4390.6(4)$ & 2161.74(11) & 3999.7(3) & $7827.0(8)$ \\
\hline $\mathrm{D}_{\mathrm{c}}\left(\mathrm{Mgm}^{-3}\right)$ & 1.161 & 1.253 & 1.248 & 1.290 \\
\hline$\mu\left(\mathrm{mm}^{-1}\right)$ & 1.363 & 2.195 & 2.367 & 2.421 \\
\hline $\mathrm{F}(000)$ & 1592 & 818 & 1508 & 3048 \\
\hline Reflns collected & 156409 & 67276 & 125825 & 44787 \\
\hline Unique reflns & 15425 & 9921 & 18071 & 8939 \\
\hline Parameters & 942 & 461 & 734 & 417 \\
\hline Goodness of fit & 1.029 & 1.151 & 1.078 & 1.042 \\
\hline$\theta$ range (deg) & $\begin{array}{c}2.902 \text { to } \\
25.000\end{array}$ & $\begin{array}{c}2.890 \text { to } \\
27.570\end{array}$ & $\begin{array}{l}2.866 \text { to } \\
27.536\end{array}$ & 1.399 to 27.473 \\
\hline $\mathrm{R}_{1}(I>2 \sigma(I))$ & 0.0714 & 0.0514 & 0.0978 & 0.0294 \\
\hline $\mathrm{wR}_{2}(I>2 \sigma(I))$ & 0.1652 & 0.1607 & 0.2370 & 0.0751 \\
\hline $\mathrm{R}$ (int) & 0.1181 & 0.0528 & 0.0981 & 0.0877 \\
\hline \multirow{2}{*}{$\begin{array}{l}\text { Largest diff. peak } \\
\left.\text { and hole (e. } \AA^{-3}\right)\end{array}$} & 0.813 & 2.729 & 2.811 & 1.578 \\
\hline & -0.685 & -1.846 & -1.807 & -1.125 \\
\hline
\end{tabular}




\section{Kinetic isotope effect experiment}

Synthesis of 2a-d $d_{1}$ :

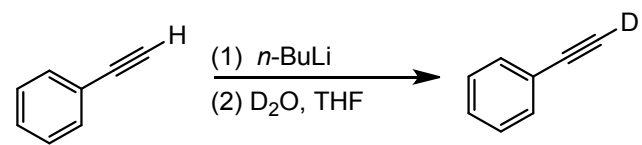

$n$-BuLi (2.5 M, $17.5 \mathrm{~mL}, 43.7 \mathrm{mmol})$ was added dropwise to a solution of phenylacetylene $(4.0 \mathrm{~mL}, 36.4 \mathrm{mmol})$ in anhydrous THF $(30 \mathrm{~mL})$ under $\operatorname{Ar}$ at $0{ }^{\circ} \mathrm{C}$ and stirred at room temperature for 1 hour. Then $\mathrm{D}_{2} \mathrm{O}(16.0 \mathrm{~mL})$ was added and stirred for an additional 2 hour. The mixture was extracted with ether $(3 \times 4.0 \mathrm{~mL})$, and the extract was dried over anhydrous sodium sulfate and evaporated to dryness to give phenylacetylene- $d_{1}(3.0 \mathrm{~g}, 80 \%)$. By comparing the ${ }^{1} \mathrm{H}$ NMR with phenylacetylene, it can be demonstrated that the alkynyl terminal hydrogen is replaced by deuterium. ${ }^{1} \mathrm{H}$ NMR $\left(500 \mathrm{MHz}, \mathrm{C}_{6} \mathrm{D} 6\right): \delta 7.37(\mathrm{dd}, J=8.0,1.5 \mathrm{~Hz}, 2 \mathrm{H})$, $6.89-6.86(\mathrm{~m}, 3 \mathrm{H})$.

\section{Kinetic isotope effect experiment of C-H/C-D}

In a glovebox, a solution of $1 \mathrm{a}(22.0 \mathrm{mg}, 0.015 \mathrm{mmol}, 5 \mathrm{~mol} \%)$ in toluene- $d_{8}(0.4 \mathrm{~mL})$ was divided equally into two J-Young NMR tubes. To one tube was added phenylacetylene ( $43.0 \mathrm{mg}, 0.30 \mathrm{mmol}, 1.0$ equiv.), and to the other tube was added phenylacetylene- $d_{1}(43.0 \mathrm{mg}, 0.30 \mathrm{mmol}, 1.0$ equiv.). The two tubes was stirred for 5 min. Then $\mathrm{HSi}(\mathrm{OEt})_{3}(97.8 \mathrm{mg}, 0.60 \mathrm{mmol}, 2.0$ equiv. $)$ was divided equally into two tubes. These tubes were sealed, taken out of the glovebox, heated at $110{ }^{\circ} \mathrm{C}$. The conversion was calculated by comparing the ${ }^{1} \mathrm{H}$ NMR integration of $\mathrm{CH}_{2}$ group in the starting material $(3.73 \mathrm{ppm}), \mathrm{Si}(\mathrm{OEt})_{4}(3.81 \mathrm{ppm})$, and the product $(3.92 \mathrm{ppm})$. A KIE value of 3.83 was found in these side-by-side reactions.
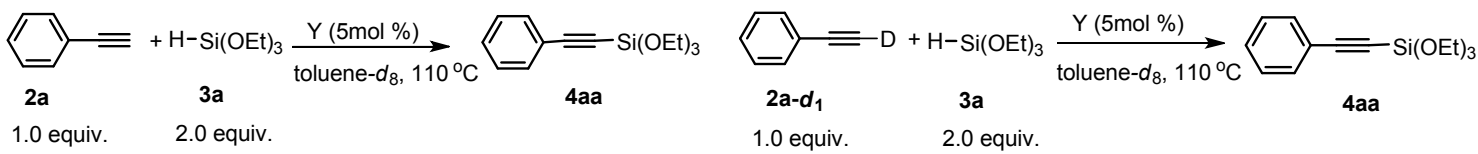

$$
K_{\mathrm{H}} / K_{\mathrm{D}}=1.8428 / 0.4810=3.83
$$
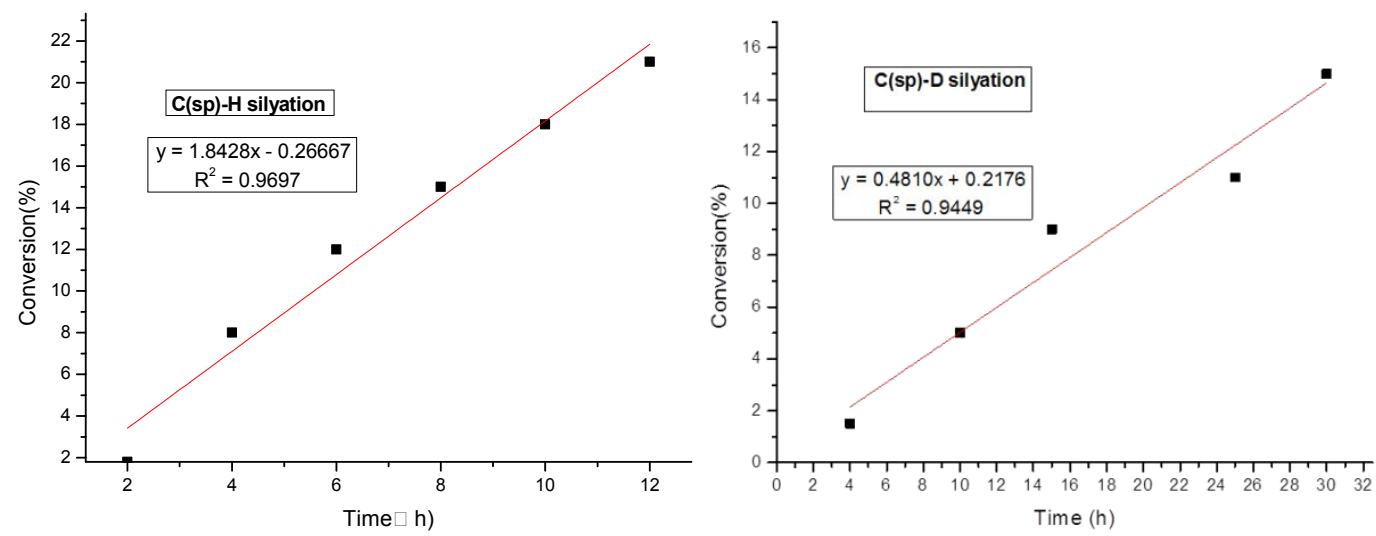

C-H and C-D silylation monitored by ${ }^{1} \mathbf{H}$ NMR $\left(500 \mathrm{MHz}\right.$, toluene- $\left.d_{8}\right)$. 


\section{Compound data}<smiles>OCC#CCOc1ccccc1</smiles>

Triethoxy(phenylethynyl)silane ${ }^{1}$ : Colorless oil. Bp: $115-120{ }^{\circ} \mathrm{C} / 10 \mathrm{~Pa} .{ }^{1} \mathrm{H}$ NMR $(500$ $\left.\mathrm{MHz}, \mathrm{CDCl}_{3}, \mathrm{ppm}\right): \delta 7.52-7.51(\mathrm{~m}, 2 \mathrm{H}), 7.35-7.30(\mathrm{~m}, 3 \mathrm{H}), 3.94(\mathrm{q}, J=7.0 \mathrm{~Hz}, 6 \mathrm{H}$, $\left.-\mathrm{OCH}_{2}\right), 1.29$ (t, $\left.J=7.0 \mathrm{~Hz}, 9 \mathrm{H},-\mathrm{OCH}_{2} \mathrm{CH}_{3}\right) .{ }^{13} \mathrm{C} \mathrm{NMR}\left(125 \mathrm{MHz}, \mathrm{CDCl}_{3}, \mathrm{ppm}\right): \delta$ 132.7, 129.6, 128.6, 122.3, $104.5(\mathrm{C} \equiv C \mathrm{CSi}), 85.4(\mathrm{C} \equiv \mathrm{CSi}), 59.4\left(-\mathrm{OCH}_{2}\right), 18.4(-$ $\mathrm{OCH}_{2} \mathrm{CH}_{3}$ ). HRMS (ESI): calcd for $\mathrm{C}_{14} \mathrm{H}_{20} \mathrm{O}_{3} \mathrm{Si}[\mathrm{M}+\mathrm{H}]^{+} 265.1254$, found 265.1252 .<smiles>CCO[As](OCC)(OCC)[N+](=O)C=Cc1ccccc1</smiles>

Diethoxy(methyl)(phenylethynyl)silane ${ }^{2}$ : Colorless oil. Bp: $125-130{ }^{\circ} \mathrm{C} / 10 \mathrm{~Pa} .{ }^{1} \mathrm{H}$ NMR (500 MHz, $\left.\mathrm{CDCl}_{3}, \mathrm{ppm}\right): \delta$ 7.51-7.49 (m, 2H), 7.35-7.25 (m, 3H), 3.93-3.89 (m, $\left.4 \mathrm{H},-\mathrm{OCH} H_{2}\right), 1.28$ (t, $\left.J=7.0 \mathrm{~Hz}, 6 \mathrm{H},-\mathrm{OCH}_{2} \mathrm{CH}_{3}\right), 0.33(\mathrm{~s}, 3 \mathrm{H}, \mathrm{Me}) .{ }^{13} \mathrm{C}$ NMR $(125$ $\left.\mathrm{MHz}, \mathrm{CDCl}_{3}, \mathrm{ppm}\right): \delta 132.6,129.5,128.6,122.6,104.6(\mathrm{C} \equiv C \mathrm{CSi}), 88.9(C \equiv \mathrm{CSi}), 59.4$ $\left(-\mathrm{OCH}_{2}\right), 18.5\left(-\mathrm{OCH}_{2} \mathrm{CH}_{3}\right),-1.78(\mathrm{SiMe})$. HRMS (ESI): calcd for $\mathrm{C}_{13} \mathrm{H}_{18} \mathrm{O}_{2} \mathrm{Si}$ $[\mathrm{M}+\mathrm{H}]^{+} 235.1154$, found 235.1157 .

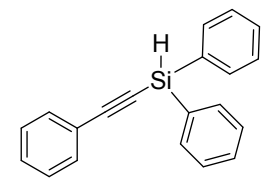

Diphenyl(phenylethynyl)silane ${ }^{3}$ White solid. Purification via column chromatography on silica gel (hexane/EtOAc $=20 / 1$, v/v). ${ }^{1} \mathrm{H}$ NMR $(500 \mathrm{MHz}$, $\left.\mathrm{CDCl}_{3}, \mathrm{ppm}\right): \delta 7.73(\mathrm{dd}, J=1.5,8.0 \mathrm{~Hz}, 4 \mathrm{H}), 7.56(\mathrm{dd}, J=1.5,8.0 \mathrm{~Hz}, 2 \mathrm{H}), 7.45-$ $7.38(\mathrm{~m}, 6 \mathrm{H}), 7.36-7.33(\mathrm{~m}, 3 \mathrm{H}), 5.30(\mathrm{~s}, 1 \mathrm{H}, \mathrm{Si} H) .{ }^{13} \mathrm{C}$ NMR $\left(125 \mathrm{MHz}, \mathrm{CDCl}_{3}\right.$, ppm): $\delta$ 135.6, 132.6, 132.5, 130.6, 129.6, 128.7, 128.6, 122.9, $110.0(\mathrm{C} \equiv C \mathrm{Si}), 87.6$ $(C \equiv \mathrm{CSi})$. HRMS (ESI): calcd for $\mathrm{C}_{20} \mathrm{H}_{16} \mathrm{Si}[\mathrm{M}+\mathrm{H}]^{+} 285.1100$, found 285.1109 .

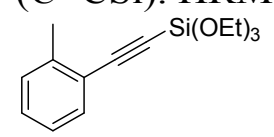

Triethoxy(o-tolylethynyl)silane: Colorless oil. Bp: $116-121{ }^{\circ} \mathrm{C} / 10 \mathrm{~Pa} .{ }^{1} \mathrm{H}$ NMR $(500$ $\left.\mathrm{MHz}, \mathrm{CDCl}_{3}, \mathrm{ppm}\right) \delta 7.49(\mathrm{~d}, J=7.5 \mathrm{~Hz}, 1 \mathrm{H}), 7.27-7.24(\mathrm{~m}, 1 \mathrm{H}), 7.20(\mathrm{~d}, J=8.0 \mathrm{~Hz}$, $1 \mathrm{H}), 7.14(\mathrm{t}, J=7.5 \mathrm{~Hz}, 1 \mathrm{H}), 3.95\left(\mathrm{q}, J=7.0 \mathrm{~Hz}, 6 \mathrm{H},-\mathrm{OCH}_{2}\right), 2.46\left(\mathrm{~s}, 3 \mathrm{H},-\mathrm{CH}_{3}\right)$, 1.29 (t, $\left.J=7.0 \mathrm{~Hz}, 9 \mathrm{H},-\mathrm{OCH}_{2} \mathrm{CH}_{3}\right) .{ }^{13} \mathrm{C} \mathrm{NMR}\left(125 \mathrm{MHz}, \mathrm{CDCl}_{3}, \mathrm{ppm}\right): \delta 141.4$, 133.1, 129.8, 129.6, 125.9, 122.1, $103.4(\mathrm{C} \equiv C \mathrm{CSi}), 89.3(\mathrm{C} \equiv \mathrm{CSi}), 59.4\left(-\mathrm{OCH}_{2}\right), 21.0$ $\left(2-\mathrm{CH}_{3} \mathrm{C}_{6} \mathrm{H}_{4}\right), 18.4\left(-\mathrm{OCH}_{2} \mathrm{CH}_{3}\right)$. HRMS (ESI): calcd for $\mathrm{C}_{15} \mathrm{H}_{22} \mathrm{O}_{3} \mathrm{Si}[\mathrm{M}+\mathrm{H}]^{+}$ 279.1411 , found 279.1417 .

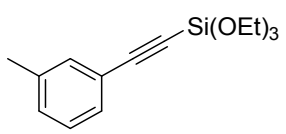


$\operatorname{Triethoxy}\left(m\right.$-tolylethynyl)silane: Colorless oil. Bp: $120-125{ }^{\circ} \mathrm{C} / 10 \mathrm{~Pa} .{ }^{1} \mathrm{H}$ NMR $(500$ $\left.\mathrm{MHz}, \mathrm{CDCl}_{3}, \mathrm{ppm}\right): \delta 7.34(\mathrm{~s}, 1 \mathrm{H}), 7.32(\mathrm{~d}, J=7.5 \mathrm{~Hz}, 1 \mathrm{H}), 7.22-7.16(\mathrm{~m}, 2 \mathrm{H}), 3.94$ (q, $\left.J=7.0 \mathrm{~Hz}, 6 \mathrm{H},-\mathrm{OCH}_{2}\right), 2.32\left(\mathrm{~s}, 3 \mathrm{H},-\mathrm{CH}_{3}\right), 1.29$ (t, $J=7.0 \mathrm{~Hz}, 9 \mathrm{H},-\mathrm{OCH}_{2} \mathrm{CH}_{3}$ ). ${ }^{13} \mathrm{C} \mathrm{NMR}\left(125 \mathrm{MHz}, \mathrm{CDCl}_{3}, \mathrm{ppm}\right): \delta$ 138.4, 133.3, 130.6, 129.9, 128.6, 122.1, 104.8 $(\mathrm{C} \equiv C \mathrm{CSi}), 85.0(\mathrm{C} \equiv \mathrm{CSi}), 59.4\left(-\mathrm{OCH}_{2}\right), 21.6\left(3-\mathrm{CH}_{3} \mathrm{C}_{6} \mathrm{H}_{4}\right), 18.6\left(-\mathrm{OCH}_{2} \mathrm{CH}_{3}\right) . \mathrm{HRMS}$ (ESI): calcd for $\mathrm{C}_{15} \mathrm{H}_{22} \mathrm{O}_{3} \mathrm{Si}[\mathrm{M}+\mathrm{H}]^{+} 279.1411$, found 279.1401 .

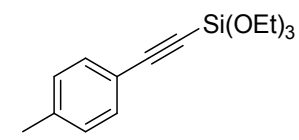

Triethoxy(p-tolylethynyl)silane ${ }^{3}$ : Colorless oil. Bp: $118-123{ }^{\circ} \mathrm{C} / 10 \mathrm{~Pa} .{ }^{1} \mathrm{H}$ NMR $(500$ $\left.\mathrm{MHz}, \mathrm{CDCl}_{3}, \mathrm{ppm}\right): \delta 7.41(\mathrm{~d}, J=8.0 \mathrm{~Hz}, 2 \mathrm{H}), 7.12(\mathrm{~d}, J=8.0 \mathrm{~Hz}, 2 \mathrm{H}), 3.93(\mathrm{q}, J=$ $\left.7.0 \mathrm{~Hz}, 6 \mathrm{H},-\mathrm{OCH}_{2}\right), 2.35\left(\mathrm{~s}, 3 \mathrm{H}, 4-\mathrm{CH}_{3} \mathrm{C}_{6} \mathrm{H}_{4}\right), 1.29\left(\mathrm{t}, J=7.0 \mathrm{~Hz}, 9 \mathrm{H},-\mathrm{OCH}_{2} \mathrm{CH}_{3}\right)$. ${ }^{13} \mathrm{C} \mathrm{NMR}$ (125 MHz, $\left.\mathrm{CDCl}_{3}, \mathrm{ppm}\right): \delta 132.6,129.4,127.1,119.2,104.8(\mathrm{C} \equiv C \mathrm{CSi}), 85.6$ $(C \equiv \mathrm{CSi}), 59.4\left(-\mathrm{OCH}_{2}\right), 21.9\left(\mathrm{CH}_{3}\right), 18.4\left(-\mathrm{OCH}_{2} \mathrm{CH}_{3}\right)$. HRMS (ESI): calcd for $\mathrm{C}_{15} \mathrm{H}_{22} \mathrm{O}_{3} \mathrm{Si}[\mathrm{M}+\mathrm{H}]^{+} 279.1411$, found 279.1408 .

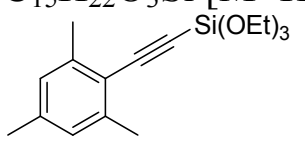

Triethoxy(mesitylethynyl)silane: Colorless oil. Bp: $126-131{ }^{\circ} \mathrm{C} / 10 \mathrm{~Pa} .{ }^{1} \mathrm{H}$ NMR $(500$ $\left.\mathrm{MHz}, \mathrm{CDCl}_{3}, \mathrm{ppm}\right): \delta 6.86(\mathrm{~s}, 2 \mathrm{H}), 3.95\left(\mathrm{q}, J=7.0 \mathrm{~Hz}, 6 \mathrm{H},-\mathrm{OCH}_{2}\right), 2.42(\mathrm{~s}, 6 \mathrm{H},-$ $\left.\mathrm{CH}_{3}\right), 2.28\left(\mathrm{~s}, 3 \mathrm{H},-\mathrm{CH}_{3}\right), 1.29\left(\mathrm{t}, J=7.0 \mathrm{~Hz}, 9 \mathrm{H},-\mathrm{OCH}_{2} \mathrm{CH}_{3}\right) .{ }^{13} \mathrm{C}$ NMR (125 MHz, $\left.\mathrm{CDCl}_{3}, \mathrm{ppm}\right): \delta 141.5,139.2,128.0,119.2,102.6(\mathrm{C} \equiv C \mathrm{CSi}), 92.8(C \equiv \mathrm{CSi}), 59.4(-$ $\left.\mathrm{OCH}_{2}\right), 21.7\left(-\mathrm{CH}_{3}\right), 21.3\left(-\mathrm{CH}_{3}\right), 18.4\left(-\mathrm{OCH}_{2} \mathrm{CH}_{3}\right)$. HRMS (ESI): calcd for $\mathrm{C}_{17} \mathrm{H}_{26} \mathrm{O}_{3} \mathrm{Si}[\mathrm{M}+\mathrm{H}]^{+}$307.1724, found 307.1733.

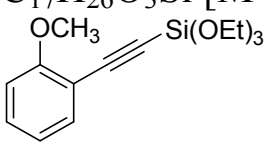

Triethoxy(2-methoxyphenyl)silane: Colorless oil. Bp: 125-130 ${ }^{\circ} \mathrm{C} / 10 \mathrm{~Pa} .{ }^{1} \mathrm{H}$ NMR $\left(500 \mathrm{MHz}, \mathrm{CDCl}_{3}, \mathrm{ppm}\right): \delta 7.47(\mathrm{dd}, J=7.5,1.5 \mathrm{~Hz}, 1 \mathrm{H}), 7.33-7.30(\mathrm{~m}, 1 \mathrm{H}), 6.90-$ $6.85(\mathrm{~m}, 2 \mathrm{H}), 3.95\left(\mathrm{q}, J=7.0 \mathrm{~Hz}, 6 \mathrm{H},-\mathrm{OCH}_{2}\right), 3.86\left(\mathrm{~s}, 3 \mathrm{H},-\mathrm{OCH}_{3}\right), 1.29$ (t, $J=7.0$ $\left.\mathrm{Hz}, 9 \mathrm{H},-\mathrm{OCH}_{2} \mathrm{CH}_{3}\right) .{ }^{13} \mathrm{C} \mathrm{NMR}\left(125 \mathrm{MHz}, \mathrm{CDCl}_{3}, \mathrm{ppm}\right): \delta 161.5,134.6,131.1,120.6$, 111.6, 111.1, 101.1 (C $\equiv C \mathrm{Si}), 89.5(C \equiv \mathrm{CSi}), 59.4\left(-\mathrm{OCH}_{2}\right), 56.0\left(-\mathrm{OCH}_{3}\right), 18.4$ ($\mathrm{OCH}_{2} \mathrm{CH}_{3}$ ). HRMS (ESI): calcd for $\mathrm{C}_{15} \mathrm{H}_{22} \mathrm{O}_{4} \mathrm{Si}[\mathrm{M}+\mathrm{H}]^{+} 295.1360$, found 283.1366.<smiles>COc1ccc(C#COc2ccccc2)cc1</smiles>

Triethoxy((4-methoxyphenyl)ethynyl)silane: Colorless oil. Bp: $127-132{ }^{\circ} \mathrm{C} / 10 \mathrm{~Pa} \cdot{ }^{1} \mathrm{H}$ NMR (500 MHz, $\left.\mathrm{CDCl}_{3}, \mathrm{ppm}\right): \delta 7.45(\mathrm{~d}, J=8.5 \mathrm{~Hz}, 2 \mathrm{H}), 6.83(\mathrm{~d}, J=8.5 \mathrm{~Hz}, 2 \mathrm{H})$, $3.93\left(\mathrm{q}, J=7.0 \mathrm{~Hz}, 6 \mathrm{H},-\mathrm{OCH}_{2}\right), 3.81\left(\mathrm{~s}, 3 \mathrm{H},-\mathrm{OCH}_{3}\right), 1.29(\mathrm{t}, J=7.0 \mathrm{~Hz}, 9 \mathrm{H},-$ $\left.\mathrm{OCH}_{2} \mathrm{CH}_{3}\right) .{ }^{13} \mathrm{C}$ NMR $\left(125 \mathrm{MHz}, \mathrm{CDCl}_{3}, \mathrm{ppm}\right): \delta 160.7,134.3,128.5,114.3,104.8$ $(\mathrm{C} \equiv C \mathrm{CSi}), 83.9(\mathrm{C} \equiv \mathrm{CSi}), 59.5\left(-\mathrm{OCH}_{2}\right), 55.6\left(-\mathrm{OCH}_{3}\right), 18.4\left(-\mathrm{OCH}_{2} \mathrm{CH}_{3}\right) . \mathrm{HRMS}(\mathrm{ESI})$ : calcd for $\mathrm{C}_{15} \mathrm{H}_{22} \mathrm{O}_{4} \mathrm{Si}[\mathrm{M}+\mathrm{H}]^{+} 295.1360$, found 295.1369 .

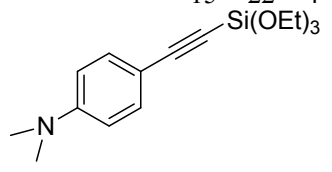

$N$, $N$-dimethyl-4-((triethoxysilyl)ethynyl)aniline: Colorless oil. Bp: $135-138{ }^{\circ} \mathrm{C} / 10 \mathrm{~Pa}$. ${ }^{1} \mathrm{H}$ NMR $\left(500 \mathrm{MHz}, \mathrm{CDCl}_{3}, \mathrm{ppm}\right): \delta 7.38(\mathrm{~d}, J=9.0 \mathrm{~Hz}, 2 \mathrm{H}), 6.59(\mathrm{~d}, J=9.0 \mathrm{~Hz}$, $2 \mathrm{H}), 3.93\left(\mathrm{q}, J=7.0 \mathrm{~Hz}, 6 \mathrm{H},-\mathrm{OCH}_{2}\right), 2.98\left(\mathrm{~s}, 6 \mathrm{H},-\mathrm{N}\left(\mathrm{CH}_{3}\right)_{2}\right), 1.28(\mathrm{t}, J=7.0 \mathrm{~Hz}, 9 \mathrm{H}$, 
$\left.-\mathrm{OCH}_{2} \mathrm{CH}_{3}\right) .{ }^{13} \mathrm{C} \mathrm{NMR}\left(125 \mathrm{MHz}, \mathrm{CDCl}_{3}, \mathrm{ppm}\right): \delta 151.0,134.0,111.8,108.7,106.5$ $(\mathrm{C} \equiv C \mathrm{CSi}), 82.6(\mathrm{C} \equiv \mathrm{CSi}), 59.3\left(-\mathrm{OCH}_{2}\right), 40.5\left(-\mathrm{N}\left(\mathrm{CH}_{3}\right)_{2}\right), 18.4\left(-\mathrm{OCH}_{2} \mathrm{CH}_{3}\right) . \mathrm{HRMS}$ (ESI): calcd for $\mathrm{C}_{16} \mathrm{H}_{25} \mathrm{O}_{3} \mathrm{SiN}[\mathrm{M}+\mathrm{H}]^{+} 308.1676$, found 308.1677 .

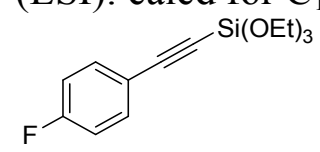

Triethoxy((4-fluorophenyl)ethynyl)silane ${ }^{3}$ : Colorless oil. Bp: $120-125{ }^{\circ} \mathrm{C} / 10 \mathrm{~Pa} .{ }^{1} \mathrm{H}$ NMR $\left(500 \mathrm{MHz}, \mathrm{CDCl}_{3}, \mathrm{ppm}\right): \delta 7.50(\mathrm{dd}, J=9.0,5.0 \mathrm{~Hz}, 2 \mathrm{H}), 7.01$ (t, $J=9.0 \mathrm{~Hz}$, $2 \mathrm{H}), 3.93\left(\mathrm{q}, J=7.0 \mathrm{~Hz}, 6 \mathrm{H},-\mathrm{OCH}_{2}\right), 1.29$ (t, $\left.J=7.0 \mathrm{~Hz}, 9 \mathrm{H},-\mathrm{OCH}_{2} \mathrm{CH}_{3}\right) .{ }^{13} \mathrm{C} \mathrm{NMR}$ $\left(125 \mathrm{MHz}, \mathrm{CDCl}_{3}, \mathrm{ppm}\right): \delta 163.4(\mathrm{~d}, J=250.0 \mathrm{~Hz}), 134.7(\mathrm{~d}, J=8.7 \mathrm{~Hz}), 118.4$, $116.0(\mathrm{~d}, J=21.2 \mathrm{~Hz}), 103.2(\mathrm{C} \equiv C \mathrm{Si}), 85.3(C \equiv \mathrm{CSi}), 59.5 \quad\left(-\mathrm{OCH}_{2}\right), 18.4 \quad(-$ $\mathrm{OCH}_{2} \mathrm{CH}_{3}$ ). HRMS (ESI): calcd for $\mathrm{C}_{14} \mathrm{H}_{19} \mathrm{O}_{3} \mathrm{SiF}[\mathrm{M}+\mathrm{H}]^{+} 283.1160$, found 283.1153.<smiles>COC#Cc1ccc(Cl)cc1</smiles>

Triethoxy((4-chlorophenyl)ethynyl)silane ${ }^{3}$ : Colorless oil. Bp: $128-133{ }^{\circ} \mathrm{C} / 10 \mathrm{~Pa} \cdot{ }^{1} \mathrm{H}$ NMR (500 MHz, $\left.\mathrm{CDCl}_{3}, \mathrm{ppm}\right): \delta 7.44(\mathrm{~d}, J=8.5 \mathrm{~Hz}, 2 \mathrm{H}), 7.29(\mathrm{~d}, J=8.5 \mathrm{~Hz}, 2 \mathrm{H})$, $3.93\left(\mathrm{q}, J=7.0 \mathrm{~Hz}, 6 \mathrm{H},-\mathrm{OCH}_{2}\right), 1.29\left(\mathrm{t}, J=7.0 \mathrm{~Hz}, 9 \mathrm{H},-\mathrm{OCH}_{2} \mathrm{CH}_{3}\right) \cdot{ }^{13} \mathrm{C}$ NMR $(125$ $\left.\mathrm{MHz}, \mathrm{CDCl}_{3}, \mathrm{ppm}\right): \delta 135.8,133.9,129.0,120.8,103.0(\mathrm{C} \equiv C \mathrm{Si}), 86.7(C \equiv \mathrm{CSi}), 59.5$ $\left(-\mathrm{OCH}_{2}\right), 18.4\left(-\mathrm{OCH}_{2} \mathrm{CH}_{3}\right)$. HRMS (ESI): calcd for $\mathrm{C}_{14} \mathrm{H}_{19} \mathrm{O}_{3} \mathrm{SiCl}[\mathrm{M}+\mathrm{H}]^{+}$299.0865, found 299.0867.

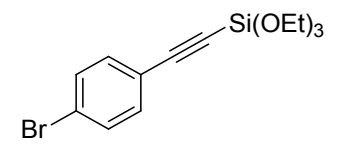

Triethoxy((4-bromophenyl)ethynyl)silane ${ }^{3}$ : Colorless oil. Bp: $132-137{ }^{\circ} \mathrm{C} / 10 \mathrm{~Pa} \cdot{ }^{1} \mathrm{H}$ NMR (500 MHz, $\left.\mathrm{CDCl}_{3}, \mathrm{ppm}\right): \delta 7.46(\mathrm{~d}, J=8.5 \mathrm{~Hz}, 2 \mathrm{H}), 7.37(\mathrm{~d}, J=8.5 \mathrm{~Hz}, 2 \mathrm{H})$, $3.93\left(\mathrm{q}, J=7.0 \mathrm{~Hz}, 6 \mathrm{H},-\mathrm{OCH}_{2}\right), 1.29\left(\mathrm{t}, J=7.0 \mathrm{~Hz}, 9 \mathrm{H},-\mathrm{OCH}_{2} \mathrm{CH}_{3}\right) .{ }^{13} \mathrm{C}$ NMR $(125$ $\left.\mathrm{MHz}, \mathrm{CDCl}_{3}, \mathrm{ppm}\right): \delta 134.1,132.0,124.1,121.2,102.9(\mathrm{C} \equiv C \mathrm{CSi}), 86.9(C \equiv \mathrm{CSi}), 59.5$ $\left(-\mathrm{OCH}_{2}\right), 18.3\left(-\mathrm{OCH}_{2} \mathrm{CH}_{3}\right)$. HRMS (ESI): calcd for $\mathrm{C}_{14} \mathrm{H}_{19} \mathrm{O}_{3} \mathrm{SiBr}[\mathrm{M}+\mathrm{H}]^{+}$343.0360, found 343.0352 .<smiles></smiles>

Triethoxy((4-(trifluoromethyl)phenyl)ethynyl)silane: Colorless oil. Bp: $125-130{ }^{\circ} \mathrm{C} / 10$ Pa. ${ }^{1} \mathrm{H}$ NMR (500 MHz, $\mathrm{CDCl}_{3}$, ppm): $\delta 7.62(\mathrm{~d}, J=8.0 \mathrm{~Hz}, 2 \mathrm{H}), 7.58(\mathrm{~d}, J=8.0 \mathrm{~Hz}$, $2 \mathrm{H}), 3.94\left(\mathrm{q}, J=7.0 \mathrm{~Hz}, 6 \mathrm{H},-\mathrm{OCH}_{2}\right), 1.29\left(\mathrm{t}, J=7.0 \mathrm{~Hz}, 9 \mathrm{H},-\mathrm{OCH}_{2} \mathrm{CH}_{3}\right) .{ }^{13} \mathrm{C} \mathrm{NMR}$ (125 MHz, $\left.\mathrm{CDCl}_{3}, \mathrm{ppm}\right): \delta 132.9,131.3(\mathrm{q}, J=32.5 \mathrm{~Hz}), 127.3,125.6(\mathrm{q}, J=3.7 \mathrm{~Hz})$, $124.1\left(\mathrm{q}, J=271.2 \mathrm{~Hz}, C \mathrm{~F}_{3}\right), 102.3(\mathrm{C} \equiv C \mathrm{CSi}), 88.3(C \equiv \mathrm{CSi}), 59.5\left(-\mathrm{OCH}_{2}\right), 18.4(-$ $\mathrm{OCH}_{2} \mathrm{CH}_{3}$ ). HRMS (ESI): calcd for $\mathrm{C}_{15} \mathrm{H}_{19} \mathrm{O}_{3} \mathrm{SiF}_{3}[\mathrm{M}+\mathrm{H}]^{+} 333.1128$, found 333.1135.<smiles>CO[SiH3]C#CC1=CCCCC1</smiles>

Triethoxy((1-cyclohexenyl)ethynyl)silane: Colorless oil. Bp: $115-120{ }^{\circ} \mathrm{C} / 10 \mathrm{~Pa} \cdot{ }^{1} \mathrm{H}$ NMR (500 MHz, $\left.\mathrm{CDCl}_{3}, \mathrm{ppm}\right): \delta 6.28-6.27(\mathrm{~m}, 1 \mathrm{H},=\mathrm{CH}), 3.88(\mathrm{q}, J=7.0 \mathrm{~Hz}, 6 \mathrm{H},-$ $\left.\mathrm{OCH}_{2}\right), 2.15-2.12\left(\mathrm{~m}, 2 \mathrm{H}, \mathrm{CH}_{2} \mathrm{C}=\mathrm{C}\right), 2.11-2.07\left(\mathrm{~m}, 2 \mathrm{H}, \mathrm{CH}_{2} \mathrm{C}=\mathrm{C}\right), 1.64-1.56(\mathrm{~m}, 4 \mathrm{H}$, $\left.\mathrm{CH}_{2} \mathrm{CH}_{2}\right), 1.25\left(\mathrm{t}, J=7.0 \mathrm{~Hz}, 9 \mathrm{H},-\mathrm{OCH}_{2} \mathrm{CH}_{3}\right) .{ }^{13} \mathrm{C} \mathrm{NMR}\left(125 \mathrm{MHz}, \mathrm{CDCl}_{3}, \mathrm{ppm}\right): \delta$ $138.6(\mathrm{C}=\mathrm{CH}), 120.3(C=\mathrm{CH}), 106.9(\mathrm{C} \equiv C \mathrm{CSi}), 82.1(C \equiv \mathrm{CSi}), 59.3\left(-\mathrm{OCH}_{2}\right), 29.0$ 
$\left(\mathrm{CH}_{2}\right), 26.0\left(\mathrm{CH}_{2}\right), 22.4\left(\mathrm{CH}_{2}\right), 21.6\left(\mathrm{CH}_{2}\right), 18.3\left(-\mathrm{OCH}_{2} \mathrm{CH}_{3}\right)$. HRMS (ESI): calcd for $\mathrm{C}_{14} \mathrm{H}_{24} \mathrm{O}_{3} \mathrm{Si}[\mathrm{M}+\mathrm{H}]^{+}$269.1567, found 269.1560.

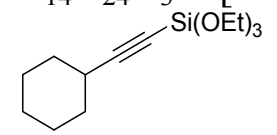

Triethoxy(cyclohexylethynyl)silane: Colorless oil. Bp: 113-118 ${ }^{\circ} \mathrm{C} / 10 \mathrm{~Pa} .{ }^{1} \mathrm{H}$ NMR $\left(500 \mathrm{MHz}, \mathrm{CDCl}_{3}, \mathrm{ppm}\right): \delta 3.87\left(\mathrm{q}, J=7.0 \mathrm{~Hz}, 6 \mathrm{H},-\mathrm{OCH}_{2}\right), 2.44(\mathrm{t}, J=8.5 \mathrm{~Hz}, 1 \mathrm{H}$, Cy), 1.81-1.79 (m, 2H, Cy), 1.71-1.70 (m, 2H, Cy), 1.54-1.48 (m, 2H, Cy), 1.32-1.26 (m, 4H, Cy), $1.25\left(\mathrm{t}, J=7.0 \mathrm{~Hz}, 9 \mathrm{H},-\mathrm{OCH}_{2} \mathrm{CH}_{3}\right) .{ }^{13} \mathrm{C} \mathrm{NMR}\left(125 \mathrm{MHz}, \mathrm{CDCl}_{3}, \mathrm{ppm}\right)$ : $\delta 111.8(\mathrm{C} \equiv C \mathrm{CSi}), 75.7(C \equiv \mathrm{CSi}), 59.2\left(-\mathrm{OCH}_{2}\right), 32.5(\mathrm{Cy}), 30.0(\mathrm{Cy}), 26.1(\mathrm{Cy}), 25.0$ (Cy), $18.3\left(-\mathrm{OCH}_{2} \mathrm{CH}_{3}\right)$. HRMS (ESI): calcd for $\mathrm{C}_{14} \mathrm{H}_{26} \mathrm{O}_{3} \mathrm{Si}[\mathrm{M}+\mathrm{H}]^{+} 271.1724$, found 271.1723 .

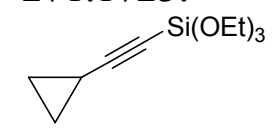

Triethoxy(cyclopropylethynyl)silane: Colorless oil. Bp: $108-113{ }^{\circ} \mathrm{C} / 10 \mathrm{~Pa} .{ }^{1} \mathrm{H}$ NMR $\left(500 \mathrm{MHz}, \mathrm{CDCl}_{3}\right): \delta 3.85$ (q, $\left.J=7.0 \mathrm{~Hz}, 6 \mathrm{H},-\mathrm{OCH}_{2}\right), 1.31-1.27(\mathrm{~m}, 1 \mathrm{H},-\mathrm{CH}), 1.24$ (t, $\left.J=7.0,9 \mathrm{H},-\mathrm{OCH}_{2} \mathrm{CH}_{3}\right), 0.82-0.77\left(\mathrm{~m}, 4 \mathrm{H},-\mathrm{CH}_{2}\right) \cdot{ }^{13} \mathrm{C} \mathrm{NMR}\left(125 \mathrm{MHz}, \mathrm{CDCl}_{3}\right.$, ppm): $\delta 110.5(\mathrm{C} \equiv C \mathrm{Si}), 71.4(C \equiv \mathrm{CSi}), 59.2\left(-\mathrm{OCH}_{2}\right), 18.3\left(-\mathrm{OCH}_{2} \mathrm{CH}_{3}\right), 9.1\left(-\mathrm{CH}_{2}\right)$, $0.5(-C H)$. HRMS (ESI): calcd for $\mathrm{C}_{11} \mathrm{H}_{20} \mathrm{O}_{3} \mathrm{Si}[\mathrm{M}+\mathrm{H}]^{+} 229.1254$, found 229.1254 .

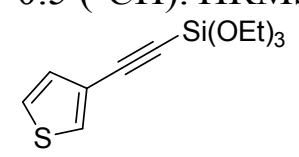

Triethoxy(thiophen-3-ylethynyl)silane: Colorless oil. Bp: 122-127 ${ }^{\circ} \mathrm{C} / 10 \mathrm{~Pa} .{ }^{1} \mathrm{H}$ NMR $\left(500 \mathrm{MHz}, \mathrm{CDCl}_{3}, \mathrm{ppm}\right): \delta 7.58\left(\mathrm{dd}, J=2.0,1.0 \mathrm{~Hz}, 1 \mathrm{H}, \mathrm{C}_{4} H_{3} \mathrm{~S}\right), 7.26\left(\mathrm{~s}, 1 \mathrm{H}, \mathrm{C}_{4} H_{3} \mathrm{~S}\right)$, $7.16\left(\mathrm{dd}, J=5.0,1.0 \mathrm{~Hz}, 1 \mathrm{H}, \mathrm{C}_{4} H_{3} \mathrm{~S}\right), 3.93\left(\mathrm{q}, J=7.0 \mathrm{~Hz}, 6 \mathrm{H},-\mathrm{OCH}_{2}\right), 1.28(\mathrm{t}, J=7.0$ $\left.\mathrm{Hz}, 9 \mathrm{H},-\mathrm{OCH}_{2} \mathrm{CH}_{3}\right) .{ }^{13} \mathrm{C} \mathrm{NMR}\left(125 \mathrm{MHz}, \mathrm{CDCl}_{3}, \mathrm{ppm}\right): \delta 131.5,130.5,125.7,121.6$, $99.3(\mathrm{C} \equiv \mathrm{CSi}), 85.3(\mathrm{C} \equiv \mathrm{CSi}), 59.5\left(-\mathrm{OCH}_{2}\right), 18.4\left(-\mathrm{OCH}_{2} \mathrm{CH}_{3}\right)$. HRMS (ESI): calcd for $\mathrm{C}_{12} \mathrm{H}_{18} \mathrm{O}_{3} \mathrm{SSi}[\mathrm{M}+\mathrm{H}]^{+} 271.0819$, found 271.0824 .

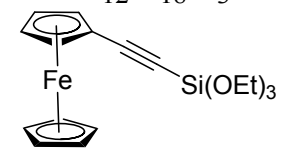

Triethoxy(ferrocenylethynyl)silane: Purplish red oil. Bp: $164-169{ }^{\circ} \mathrm{C} / 10 \mathrm{~Pa} .{ }^{1} \mathrm{H}$ NMR (500 MHz, $\left.\mathrm{CDCl}_{3}, \mathrm{ppm}\right): \delta 4.49$ (t, $\left.J=2.0 \mathrm{~Hz}, 2 \mathrm{H}, \mathrm{C}_{10} H_{9} \mathrm{Fe}\right), 4.21(\mathrm{t}, J=2.0 \mathrm{~Hz}, 2 \mathrm{H}$, $\left.\mathrm{C}_{10} H_{9} \mathrm{Fe}\right), 4.20\left(\mathrm{~s}, 5 \mathrm{H}, \mathrm{C}_{10} H_{9} \mathrm{Fe}\right), 3.93\left(\mathrm{q}, J=7.0 \mathrm{~Hz}, 6 \mathrm{H},-\mathrm{OCH}_{2}\right), 1.29$ (t, $J=7.0 \mathrm{~Hz}$, 9H, $\left.-\mathrm{OCH}_{2} \mathrm{CH}_{3}\right) .{ }^{13} \mathrm{C} \mathrm{NMR}\left(125 \mathrm{MHz}, \mathrm{CDCl}_{3}, \mathrm{ppm}\right): \delta 104.5(\mathrm{C} \equiv \mathrm{CSi}), 81.9(\mathrm{C} \equiv \mathrm{CSi})$, $72.5\left(C_{10} \mathrm{H}_{9} \mathrm{Fe}\right), 70.5\left(C_{10} \mathrm{H}_{9} \mathrm{Fe}\right), 69.5\left(C_{10} \mathrm{H}_{9} \mathrm{Fe}\right), 63.5\left(C_{10} \mathrm{H}_{9} \mathrm{Fe}\right), 59.3\left(-\mathrm{OCH}_{2}\right), 18.4$ $\left(-\mathrm{OCH}_{2} \mathrm{CH}_{3}\right)$. HRMS (ESI): calcd for $\mathrm{C}_{18} \mathrm{H}_{24} \mathrm{O}_{3} \mathrm{SiFe}[\mathrm{M}+\mathrm{H}]^{+}$373.0917, found 373.0924.

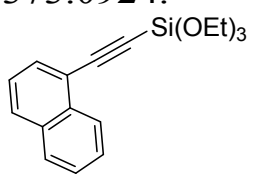

Triethoxy(naphthalen-1-ylethynyl)silane ${ }^{3}$ : Colorless oil. Bp: $145-150{ }^{\circ} \mathrm{C} / 10 \mathrm{~Pa} .{ }^{1} \mathrm{H}$ NMR (500 MHz, $\left.\mathrm{CDCl}_{3}, \mathrm{ppm}\right): \delta 8.35\left(\mathrm{~d}, J=8.5 \mathrm{~Hz}, 1 \mathrm{H}, \mathrm{C}_{10} H_{7}\right), 7.86(\mathrm{t}, J=8.0 \mathrm{~Hz}$, $\left.2 \mathrm{H}, \mathrm{C}_{10} H_{7}\right), 7.78\left(\mathrm{dd}, J=7.0,1.0 \mathrm{~Hz}, 1 \mathrm{H}, \mathrm{C}_{10} H_{7}\right), 7.60-7.57\left(\mathrm{~m}, 1 \mathrm{H}, \mathrm{C}_{10} H_{7}\right), 7.54-$ $7.51\left(\mathrm{~m}, 1 \mathrm{H}, \mathrm{C}_{10} H_{7}\right), 7.42\left(\mathrm{dd}, J=7.0,8.0 \mathrm{~Hz}, 1 \mathrm{H}, \mathrm{C}_{10} H_{7}\right), 4.01(\mathrm{q}, J=7.0 \mathrm{~Hz}, 6 \mathrm{H},-$ 
$\left.\mathrm{OCH}_{2}\right), 1.33\left(\mathrm{t}, J=7.0 \mathrm{~Hz}, 9 \mathrm{H},-\mathrm{OCH}_{2} \mathrm{CH}_{3}\right) .{ }^{13} \mathrm{C} \mathrm{NMR}\left(125 \mathrm{MHz}, \mathrm{CDCl}_{3}, \mathrm{ppm}\right): \delta$ $133.8,133.4,132.2,130.2,128.7,127.5,127.0,126.4,125.4,119.7,102.6(\mathrm{C} \equiv C \mathrm{CSi})$, $90.5(C \equiv \mathrm{CSi}), 59.6\left(-\mathrm{OCH}_{2}\right), 18.5\left(-\mathrm{OCH}_{2} \mathrm{CH}_{3}\right)$. HRMS (ESI): calcd for $\mathrm{C}_{18} \mathrm{H}_{22} \mathrm{O}_{3} \mathrm{Si}$ $[\mathrm{M}+\mathrm{H}]^{+}$315.1411, found 315.1405 .

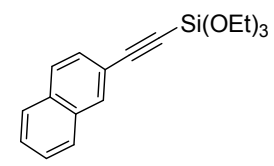

Triethoxy(naphthalen-2-ylethynyl)silane: Colorless oil. Bp: $147-152{ }^{\circ} \mathrm{C} / 10 \mathrm{~Pa} .{ }^{1} \mathrm{H}$ NMR $\left(500 \mathrm{MHz}, \mathrm{CDCl}_{3}\right): \delta 8.06\left(\mathrm{~s}, 1 \mathrm{H}, \mathrm{C}_{10} \mathrm{H}_{7}\right), 7.82-7.77\left(\mathrm{~m}, 3 \mathrm{H}, \mathrm{C}_{10} H_{7}\right), 7.54-$ $7.49\left(\mathrm{~m}, 3 \mathrm{H}, \mathrm{C}_{10} H_{7}\right), 3.97\left(\mathrm{q}, J=7.0 \mathrm{~Hz}, 6 \mathrm{H},-\mathrm{OCH}_{2}\right), 1.32(\mathrm{t}, J=7.0 \mathrm{~Hz}, 9 \mathrm{H},-$ $\left.\mathrm{OCH}_{2} \mathrm{CH}_{3}\right) .{ }^{13} \mathrm{C} \mathrm{NMR}\left(125 \mathrm{MHz}, \mathrm{CDCl}_{3}, \mathrm{ppm}\right): \delta 133.6,133.2,133.1,128.4,128.2$, 128.1, 127.5, 127.1, 126.7, 119.5, $104.8(\mathrm{C} \equiv C \mathrm{CSi}), 85.7(C \equiv \mathrm{CSi}), 59.5\left(-\mathrm{OCH}_{2}\right), 18.4$ $\left(-\mathrm{OCH}_{2} \mathrm{CH}_{3}\right.$ ). HRMS (ESI): calcd for $\mathrm{C}_{18} \mathrm{H}_{22} \mathrm{O}_{3} \mathrm{Si}[\mathrm{M}+\mathrm{H}]^{+} 315.1411$, found 315.1414 .

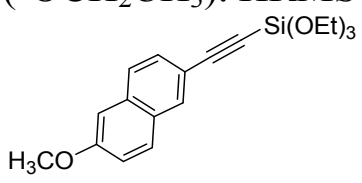

Triethoxy((6-methoxynaphthalen-2-ylethynyl)silane: Colorless oil. Bp: $158-163{ }^{\circ} \mathrm{C} / 10$ Pa. ${ }^{1} \mathrm{H}$ NMR (500 MHz, $\left.\mathrm{CDCl}_{3}, \mathrm{ppm}\right): \delta 7.98\left(\mathrm{~s}, 1 \mathrm{H}, \mathrm{C}_{10} H_{6}\right), 7.69(\mathrm{~d}, J=9.0 \mathrm{~Hz}, 1 \mathrm{H}$, $\left.\mathrm{C}_{10} H_{6}\right), 7.66\left(\mathrm{~d}, J=8.5 \mathrm{~Hz}, 1 \mathrm{H}, \mathrm{C}_{10} H_{6}\right), 7.50\left(\mathrm{dd}, J=8.5,1.5 \mathrm{~Hz}, 1 \mathrm{H}, \mathrm{C}_{10} H_{6}\right), 7.16$ $\left(\mathrm{dd}, J=9.0,2.5 \mathrm{~Hz}, 1 \mathrm{H}, \mathrm{C}_{10} H_{6}\right), 7.10\left(\mathrm{~d}, J=2.5 \mathrm{~Hz}, 1 \mathrm{H}, \mathrm{C}_{10} H_{6}\right), 3.97$ (q, $J=7.0 \mathrm{~Hz}$, $\left.6 \mathrm{H},-\mathrm{OCH}_{2}\right), 3.92\left(\mathrm{~s}, 3 \mathrm{H},-\mathrm{OCH}_{3}\right), 1.31\left(\mathrm{t}, J=7.0 \mathrm{~Hz}, 9 \mathrm{H},-\mathrm{OCH}_{2} \mathrm{CH}_{3}\right) .{ }^{13} \mathrm{C}$ NMR $(125$ $\left.\mathrm{MHz}, \mathrm{CDCl}_{3}, \mathrm{ppm}\right): \delta 159.1,135.0,133.0,129.8,129.5,128.5,127.1,119.9,117.1$, 106.2, $105.2(\mathrm{C} \equiv C \mathrm{Si}), 84.9(\mathrm{C} \equiv \mathrm{CSi}), 59.5\left(-\mathrm{OCH}_{2}\right), 55.7\left(-\mathrm{OCH}_{3}\right), 18.4\left(-\mathrm{OCH}_{2} \mathrm{CH}_{3}\right)$. HRMS (ESI): calcd for $\mathrm{C}_{19} \mathrm{H}_{24} \mathrm{O}_{4} \mathrm{Si}[\mathrm{M}+\mathrm{Na}]^{+} 367.1336$, found 367.1341 .

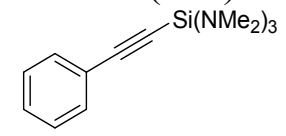

$N$-hexamethyl-1-(phenylethynyl)silanetriamine: Colorless oil. Bp: $120-125{ }^{\circ} \mathrm{C} / 10 \mathrm{~Pa}$. ${ }^{1} \mathrm{H}$ NMR (500 MHz, $\left.\mathrm{CDCl}_{3}, \mathrm{ppm}\right): \delta$ 7.49-7.47 (m, 2H), 7.29-7.27 (m, 3H), 2.53 (s, $\left.18 \mathrm{H},-\mathrm{NMe} e_{2}\right) .{ }^{13} \mathrm{C} \mathrm{NMR}\left(125 \mathrm{MHz}, \mathrm{CDCl}_{3}, \mathrm{ppm}\right): \delta 132.5,128.7,128.5,123.8,104.1$ $(\mathrm{C} \equiv C \mathrm{Si}), 90.5(C \equiv \mathrm{CSi}), 37.4\left(-\mathrm{NM} e_{2}\right)$. HRMS (ESI): calcd for $\mathrm{C}_{14} \mathrm{H}_{23} \mathrm{~N}_{3} \mathrm{Si}[\mathrm{M}+\mathrm{H}]^{+}$ 262.1734 , found 262.1738 .

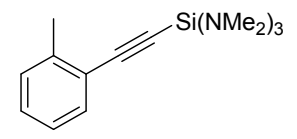

$N$-hexamethyl-1-(o-tolylethynyl))silanetriamine: Colorless oil. Bp: $123-128{ }^{\circ} \mathrm{C} / 10 \mathrm{~Pa}$. ${ }^{1} \mathrm{H}$ NMR (500 MHz, $\left.\mathrm{CDCl}_{3}, \mathrm{ppm}\right): \delta 7.46(\mathrm{~d}, J=7.5 \mathrm{~Hz}, 1 \mathrm{H}), 7.20-7.17$ (m, 2H), 7.12$7.09(\mathrm{~m}, 1 \mathrm{H}), 2.54(\mathrm{~s}, 18 \mathrm{H},-\mathrm{NMe}), 2.46\left(\mathrm{~s}, 3 \mathrm{H}, \mathrm{CH}_{3}\right) .{ }^{13} \mathrm{C} \mathrm{NMR}\left(125 \mathrm{MHz}, \mathrm{CDCl}_{3}\right.$, ppm): $\delta 140.9,132.7,129.7,128.7,125.7,123.6,102.9(\mathrm{C} \equiv C S i), 94.6(C \equiv \mathrm{CSi}), 37.4$ (-NMe $)$, $21.3\left(\mathrm{CH}_{3}\right)$. HRMS (ESI): calcd for $\mathrm{C}_{15} \mathrm{H}_{25} \mathrm{~N}_{3} \mathrm{Si}[\mathrm{M}+\mathrm{H}]^{+}$276.1891, found 276.1895 .

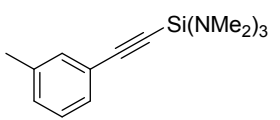

$N$-hexamethyl-1-(m-tolylethynyl))silanetriamine: Colorless oil. Bp: $122-127{ }^{\circ} \mathrm{C} / 10 \mathrm{~Pa}$. ${ }^{1} \mathrm{H}$ NMR (500 MHz, $\left.\mathrm{CDCl}_{3}, \mathrm{ppm}\right): \delta 7.31(\mathrm{~s}, 1 \mathrm{H}), 7.29(\mathrm{~d}, J=7.5 \mathrm{~Hz}, 1 \mathrm{H}), 7.15$ (t, $J$ 
$=7.5 \mathrm{~Hz}, 1 \mathrm{H}), 7.11(\mathrm{~d}, J=7.5 \mathrm{~Hz}, 1 \mathrm{H}), 2.53\left(\mathrm{~s}, 18 \mathrm{H},-\mathrm{N} M e_{2}\right), 2.31\left(\mathrm{~s}, 3 \mathrm{H}, \mathrm{CH}_{3}\right) .{ }^{13} \mathrm{C}$ NMR (125 MHz, $\left.\mathrm{CDCl}_{3}, \mathrm{ppm}\right): \delta 138.1,133.0,129.6,129.5,128.1,123.6,104.4$ $(\mathrm{C} \equiv C \mathrm{CSi}), 90.0 \quad(\mathrm{C} \equiv \mathrm{CSi}), \quad 37.4 \quad(-\mathrm{NMe}), 21.5 \quad\left(\mathrm{CH}_{3}\right)$. HRMS (ESI): calcd for $\mathrm{C}_{15} \mathrm{H}_{25} \mathrm{~N}_{3} \mathrm{Si}[\mathrm{M}+\mathrm{H}]^{+}$276.1891, found 276.1897.

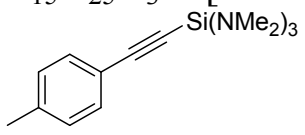

$N$-hexamethyl-1-(p-tolylethynyl))silanetriamine: Colorless oil. Bp: $125-130{ }^{\circ} \mathrm{C} / 10 \mathrm{~Pa}$. ${ }^{1} \mathrm{H}$ NMR $\left(500 \mathrm{MHz}, \mathrm{CDCl}_{3}, \mathrm{ppm}\right): \delta 7.38(\mathrm{~d}, J=7.5 \mathrm{~Hz}, 1 \mathrm{H}), 7.09(\mathrm{t}, J=8.0 \mathrm{~Hz}, 1 \mathrm{H})$, $7.11(\mathrm{~d}, J=7.5 \mathrm{~Hz}, 1 \mathrm{H}), 2.53\left(\mathrm{~s}, 18 \mathrm{H},-\mathrm{NMe} e_{2}\right), 2.33\left(\mathrm{~s}, 3 \mathrm{H}, \mathrm{CH}_{3}\right) .{ }^{13} \mathrm{C}$ NMR $(125$ $\left.\mathrm{MHz}, \mathrm{CDCl}_{3}, \mathrm{ppm}\right): \delta 138.8,132.4,129.2,120.7,104.4(\mathrm{C} \equiv C \mathrm{CSi}), 89.6(C \equiv \mathrm{CSi}), 37.4$ $(-\mathrm{NMe})$, $21.8\left(\mathrm{CH}_{3}\right)$. HRMS (ESI): calcd for $\mathrm{C}_{15} \mathrm{H}_{25} \mathrm{~N}_{3} \mathrm{Si}[\mathrm{M}+\mathrm{H}]^{+} 276.1891$, found 276.1888 .

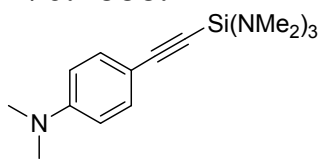

1-((4-(dimethylamino)phenyl)ethynyl)- $N$-hexamethylsilanetriamine: Colorless oil. Bp: 138-143 ${ }^{\circ} \mathrm{C} / 10 \mathrm{~Pa} .{ }^{1} \mathrm{H}$ NMR $\left(500 \mathrm{MHz}, \mathrm{CDCl}_{3}, \mathrm{ppm}\right): \delta 7.36(\mathrm{~d}, J=9.0 \mathrm{~Hz}, 1 \mathrm{H}), 7.09$ $(\mathrm{t}, J=9.0 \mathrm{~Hz}, 1 \mathrm{H}), 2.96\left(\mathrm{~s}, 3 \mathrm{H}, \mathrm{N}\left(\mathrm{CH}_{3}\right)_{2}\right), 2.53\left(\mathrm{~s}, 18 \mathrm{H},-(\mathrm{NMe})_{3}\right) .{ }^{13} \mathrm{C}$ NMR $(125$ $\left.\mathrm{MHz}, \mathrm{CDCl}_{3}, \mathrm{ppm}\right): \delta 150.5,133.6,111.9,110.6,105.7(\mathrm{C} \equiv C \mathrm{CSi}), 87.4(C \equiv \mathrm{CSi}), 40.6$ $\left(\mathrm{N}\left(\mathrm{CH}_{3}\right)_{2}\right), 37.5\left(-(\mathrm{NMe})_{3}\right)$. HRMS (ESI): calcd for $\mathrm{C}_{16} \mathrm{H}_{28} \mathrm{~N}_{4} \mathrm{Si}[\mathrm{M}+\mathrm{H}]^{+}$305.2156, found 305.2151.

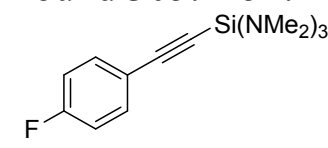

1-((4-fluorophenyl)ethynyl)- $N$-hexamethylsilanetriamine: Colorless oil. Bp: 133-138 ${ }^{\circ} \mathrm{C} / 10 \mathrm{~Pa} .{ }^{1} \mathrm{H}$ NMR $\left(500 \mathrm{MHz}, \mathrm{CDCl}_{3}, \mathrm{ppm}\right): \delta 7.46(\mathrm{dd}, J=9.0,5.5 \mathrm{~Hz}, 2 \mathrm{H}), 6.97(\mathrm{t}$, $J=9.0 \mathrm{~Hz}, 2 \mathrm{H}), 2.96\left(\mathrm{~s}, 2.53\left(\mathrm{~s}, 18 \mathrm{H},-\mathrm{NMe} e_{2}\right) .{ }^{13} \mathrm{C} \mathrm{NMR}\left(125 \mathrm{MHz}, \mathrm{CDCl}_{3}, \mathrm{ppm}\right): \delta\right.$ $162.9(\mathrm{~d}, J=248.7 \mathrm{~Hz}), 134.4(\mathrm{~d}, J=8.7 \mathrm{~Hz}), 119.9,115.7(\mathrm{~d}, J=8.7 \mathrm{~Hz}), 102.9$ $(\mathrm{C} \equiv C \mathrm{Si}), 90.2(C \equiv \mathrm{CSi}), 37.3(-\mathrm{NMe})_{2}$. HRMS (ESI): calcd for $\mathrm{C}_{14} \mathrm{H}_{22} \mathrm{~N}_{3} \mathrm{FSi}[\mathrm{M}+\mathrm{Na}]^{+}$ 302.1459 , found 302.1463 .

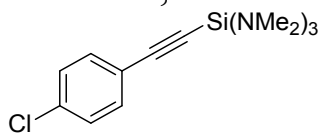

1-((4-chlorophenyl)ethynyl)- $N$-hexamethylsilanetriamine: Colorless oil. Bp: 138-142 ${ }^{\circ} \mathrm{C} / 10 \mathrm{~Pa} .{ }^{1} \mathrm{H}$ NMR $\left(500 \mathrm{MHz}, \mathrm{CDCl}_{3}, \mathrm{ppm}\right): \delta 7.40(\mathrm{~d}, J=8.5 \mathrm{~Hz}, 2 \mathrm{H}), 7.26(\mathrm{~d}, J=$ $8.5 \mathrm{~Hz}, 2 \mathrm{H}), 2.52$ (s, $\left.18 \mathrm{H},-\mathrm{NMe} e_{2}\right) .{ }^{13} \mathrm{C} \mathrm{NMR}\left(125 \mathrm{MHz}, \mathrm{CDCl}_{3}, \mathrm{ppm}\right): \delta 134.7,133.7$, 128.8, 122.2, $102.8(\mathrm{C} \equiv C \mathrm{CSi}), 91.8(C \equiv \mathrm{CSi}), 37.3\left(-\mathrm{N} M e_{2}\right)$. HRMS (ESI): calcd for $\mathrm{C}_{14} \mathrm{H}_{22} \mathrm{~N}_{3} \mathrm{ClSi}[\mathrm{M}+\mathrm{Na}]^{+}$318.1164, found 318.1165 .

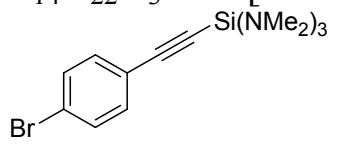

1-((4-bromophenyl)ethynyl)- $N$-hexamethylsilanetriamine: Colorless oil. Bp: 143-148 ${ }^{\circ} \mathrm{C} / 10 \mathrm{~Pa} .{ }^{1} \mathrm{H}$ NMR $\left(500 \mathrm{MHz}, \mathrm{CDCl}_{3}, \mathrm{ppm}\right): \delta 7.42(\mathrm{~d}, J=8.5 \mathrm{~Hz}, 2 \mathrm{H}), 7.34(\mathrm{~d}, J=$ $8.5 \mathrm{~Hz}, 2 \mathrm{H}), 2.52$ (s, $\left.18 \mathrm{H},-\mathrm{NMe} e_{2}\right) .{ }^{13} \mathrm{C} \mathrm{NMR}\left(125 \mathrm{MHz}, \mathrm{CDCl}_{3}, \mathrm{ppm}\right): \delta 133.9,131.7$, 122.9, 122.7, $102.8(\mathrm{C} \equiv C \mathrm{CSi}), 92.0(C \equiv \mathrm{CSi}), 37.3\left(-\mathrm{N} M e_{2}\right)$. HRMS (ESI): calcd for $\mathrm{C}_{14} \mathrm{H}_{22} \mathrm{~N}_{3} \mathrm{BrSi}[\mathrm{M}+\mathrm{Na}]^{+} 362.0659$, found 362.0664 . 
1-(cyclohexenyl)ethynyl)- $N$-hexamethylsilanetriamine: Colorless oil. Bp: 117-122 ${ }^{\circ} \mathrm{C} / 10 \mathrm{~Pa} .{ }^{1} \mathrm{H}$ NMR $\left(500 \mathrm{MHz}, \mathrm{CDCl}_{3}, \mathrm{ppm}\right): \delta 6.19-6.17(\mathrm{~m}, 1 \mathrm{H},=\mathrm{CH}), 2.48(\mathrm{~s}, 18 \mathrm{H}$, $\left.-\mathrm{NMe} e_{2}\right), 2.17-2.14\left(\mathrm{~m}, 2 \mathrm{H}, \mathrm{CH}_{2} \mathrm{C}=\mathrm{C}\right), 2.10-2.06\left(\mathrm{~m}, 2 \mathrm{H}, \mathrm{CH}_{2} \mathrm{C}=\mathrm{C}\right), 1.64-1.54(\mathrm{~m}, 4 \mathrm{H}$, $\left.\mathrm{CH}_{2} \mathrm{CH}_{2}\right) .{ }^{13} \mathrm{C}$ NMR $\left(125 \mathrm{MHz}, \mathrm{CDCl}_{3}, \mathrm{ppm}\right): \delta 136.2(\mathrm{C}=\mathrm{CH}), 121.3(\mathrm{C}=\mathrm{CH}), 106.4$ $(\mathrm{C} \equiv C \mathrm{CSi}), 87.0(\mathrm{C} \equiv \mathrm{CSi}), 37.3(-\mathrm{NMe}), 29.5\left(\mathrm{CH}_{2}\right), 26.0\left(\mathrm{CH}_{2}\right), 22.6\left(\mathrm{CH}_{2}\right), 21.8$ $\left(\mathrm{CH}_{2}\right)$. HRMS (ESI): calcd for $\mathrm{C}_{14} \mathrm{H}_{27} \mathrm{~N}_{3} \mathrm{Si}[\mathrm{M}+\mathrm{H}]^{+} 266.2047$, found 266.2047. $\mathrm{Ri}^{\mathrm{Si}\left(\mathrm{NMe}_{2}\right)_{3}}$

cyclohexylethynyl- $N$-hexamethylsilanetriamine: Colorless oil. Bp: $115-120{ }^{\circ} \mathrm{C} / 10 \mathrm{~Pa}$. ${ }^{1} \mathrm{H}$ NMR $\left(500 \mathrm{MHz}, \mathrm{CDCl}_{3}, \mathrm{ppm}\right): \delta 2.48\left(\mathrm{~s}, 18 \mathrm{H},-\mathrm{NMe} e_{2}\right), 2.44(\mathrm{~s}, 1 \mathrm{H}, \mathrm{Cy}), 2.24(\mathrm{t}, J$ $=8.5 \mathrm{~Hz}, 2 \mathrm{H}, \mathrm{Cy}), 1.52-1.49(\mathrm{~m}, 2 \mathrm{H}, \mathrm{Cy}), 1.45-1.40(\mathrm{~m}, 2 \mathrm{H}, \mathrm{Cy}), 0.90(\mathrm{t}, J=7.5 \mathrm{~Hz}$, 4H, Cy). ${ }^{13} \mathrm{C}$ NMR (125 MHz, $\left.\mathrm{CDCl}_{3}, \mathrm{ppm}\right): \delta 106.6(\mathrm{C} \equiv C \mathrm{Si}), 80.4(C \equiv \mathrm{CSi}), 37.3(-$ $\mathrm{NMe}$ ), 31.1 (Сy), 22.2 (Cy), 19.8 (Cy), 13.9 (Cy). HRMS (ESI): calcd for $\mathrm{C}_{14} \mathrm{H}_{29} \mathrm{~N}_{3} \mathrm{Si}[\mathrm{M}+\mathrm{Na}]^{+}$290.2023, found 290.2029.

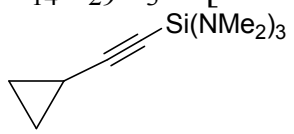

1-(cyclopropylethynyl)- $N$-hexamethylsilanetriamine: Colorless oil. Bp: $114-119{ }^{\circ} \mathrm{C} / 10$ Pa. ${ }^{1} \mathrm{H}$ NMR (500 MHz, $\left.\mathrm{CDCl}_{3}, \mathrm{ppm}\right): \delta 2.46(\mathrm{~s}, 18 \mathrm{H},-\mathrm{NMe}), 1.30-1.27(\mathrm{~m}, 1 \mathrm{H},-$ $\mathrm{CH}), 0.76-0.72\left(\mathrm{~m}, 4 \mathrm{H},-\mathrm{CH}_{2}\right) .{ }^{13} \mathrm{C} \mathrm{NMR}\left(125 \mathrm{MHz}, \mathrm{CDCl}_{3}, \mathrm{ppm}\right): \delta 109.5(\mathrm{C} \equiv \mathrm{CSi})$, $75.6(C \equiv \mathrm{CSi}), 37.3(-\mathrm{NMe}), 9.1 \quad\left(-\mathrm{CH}_{2}\right), 0.8(-\mathrm{CH})$. HRMS (ESI): calcd for $\mathrm{C}_{11} \mathrm{H}_{23} \mathrm{~N}_{3} \mathrm{Si}[\mathrm{M}+\mathrm{H}]^{+} 226.1734$, found 226.1736.

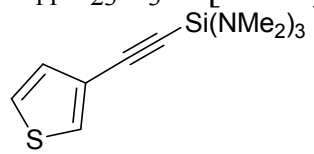

1-(thiophen-3-ylethynyl)- $N$-hexamethylsilanetriamine: Colorless oil. Bp: 119-134 ${ }^{\circ} \mathrm{C} / 10 \mathrm{~Pa} .{ }^{1} \mathrm{H}$ NMR $\left(500 \mathrm{MHz}, \mathrm{CDCl}_{3}, \mathrm{ppm}\right): \delta 7.48\left(\mathrm{dd}, J=3.0,1.0 \mathrm{~Hz}, 1 \mathrm{H}, \mathrm{C}_{4} H_{3} \mathrm{~S}\right)$, $7.22\left(\mathrm{dd}, J=5.0,3.0 \mathrm{~Hz}, 1 \mathrm{H}, \mathrm{C}_{4} H_{3} \mathrm{~S}\right), 7.14\left(\mathrm{dd}, J=5.0,1.0 \mathrm{~Hz}, 1 \mathrm{H}, \mathrm{C}_{4} H_{3} \mathrm{~S}\right), 2.52(\mathrm{~s}$, $\left.18 \mathrm{H},-\mathrm{NMe} e_{2}\right) .{ }^{13} \mathrm{C} \mathrm{NMR}\left(125 \mathrm{MHz}, \mathrm{CDCl}_{3}, \mathrm{ppm}\right): \delta 130.7,129.8,125.3,123.0,98.8$ $(\mathrm{C} \equiv C \mathrm{CSi}), 90.2(\mathrm{C} \equiv \mathrm{CSi}), 37.3(-\mathrm{NMe})_{2}$. HRMS (ESI): calcd for $\mathrm{C}_{12} \mathrm{H}_{21} \mathrm{~N}_{3} \mathrm{SSi}[\mathrm{M}+\mathrm{H}]^{+}$ 268.1298 , found 268.1299 .

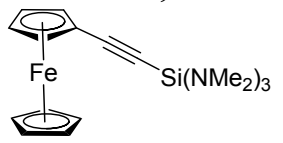

1-(ferrocenylethynyl)- $N$-hexamethylsilanetriamine: Purplish red oil. Bp: 165-170 ${ }^{\circ} \mathrm{C} / 10 \mathrm{~Pa} .{ }^{1} \mathrm{H}$ NMR $\left(500 \mathrm{MHz}, \mathrm{CDCl}_{3}, \mathrm{ppm}\right): \delta 4.44$ (t, $J=2.0 \mathrm{~Hz}, 2 \mathrm{H}, \mathrm{C}_{10} \mathrm{H}_{9} \mathrm{Fe}$ ), 4.19 $\left.\left(\mathrm{s}, 5 \mathrm{H}, \mathrm{C}_{10} H_{9} \mathrm{Fe}\right), 4.16\left(\mathrm{t}, J=2.0 \mathrm{~Hz}, 2 \mathrm{H}, \mathrm{C}_{10} H_{9} \mathrm{Fe}\right), 2.53(\mathrm{~s}, 18 \mathrm{H},-\mathrm{NMe})_{2}\right){ }^{13} \mathrm{C} \mathrm{NMR}$ $\left(125 \mathrm{MHz}, \mathrm{CDCl}_{3}, \mathrm{ppm}\right): \delta 103.2(\mathrm{C} \equiv C \mathrm{CSi}), 86.6(C \equiv \mathrm{CSi}), 72.2\left(C_{10} \mathrm{H}_{9} \mathrm{Fe}\right), 70.4$ $\left(C_{10} \mathrm{H}_{9} \mathrm{Fe}\right), 69.0\left(C_{10} \mathrm{H}_{9} \mathrm{Fe}\right), 65.4\left(C_{10} \mathrm{H}_{9} \mathrm{Fe}\right), 37.5\left(-\mathrm{N} M e_{2}\right)$. HRMS (ESI): calcd for $\mathrm{C}_{18} \mathrm{H}_{27} \mathrm{~N}_{3} \mathrm{FeSi}[\mathrm{M}+\mathrm{Na}]^{+}$392.1216, found 392.1224.

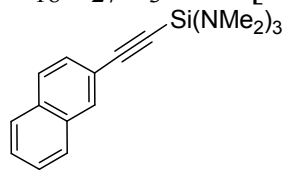

1-(naphthalen-2-ylethynyl)- $N$-hexamethylsilanetriamine: Colorless oil. Bp: 155-160 ${ }^{\circ} \mathrm{C} / 10 \mathrm{~Pa} .{ }^{1} \mathrm{H}$ NMR $\left(500 \mathrm{MHz}, \mathrm{CDCl}_{3}\right): \delta 8.01\left(\mathrm{~s}, 1 \mathrm{H}, \mathrm{C}_{10} H_{7}\right), 7.80-7.74(\mathrm{~m}, 3 \mathrm{H}$, $\left.\left.\mathrm{C}_{10} \mathrm{H}_{7}\right), 7.53-7.46\left(\mathrm{~m}, 3 \mathrm{H}, \mathrm{C}_{10} \mathrm{H}_{7}\right), 2.56(\mathrm{~s}, 18 \mathrm{H},-\mathrm{NMe})_{2}\right) .{ }^{13} \mathrm{C}$ NMR $(125 \mathrm{MHz}$, $\left.\mathrm{CDCl}_{3}, \mathrm{ppm}\right): \delta 133.3,133.2,132.4,129.2,128.1,127.0,126.8,121.2,104.5(\mathrm{C} \equiv C \mathrm{CSi})$, $90.9(C \equiv \mathrm{CSi}), 37.4\left(-\mathrm{NMe} e_{2}\right)$. HRMS (ESI): calcd for $\mathrm{C}_{18} \mathrm{H}_{25} \mathrm{~N}_{3} \mathrm{Si}[\mathrm{M}+\mathrm{H}]^{+}$312.1891, found 312.1891 . 


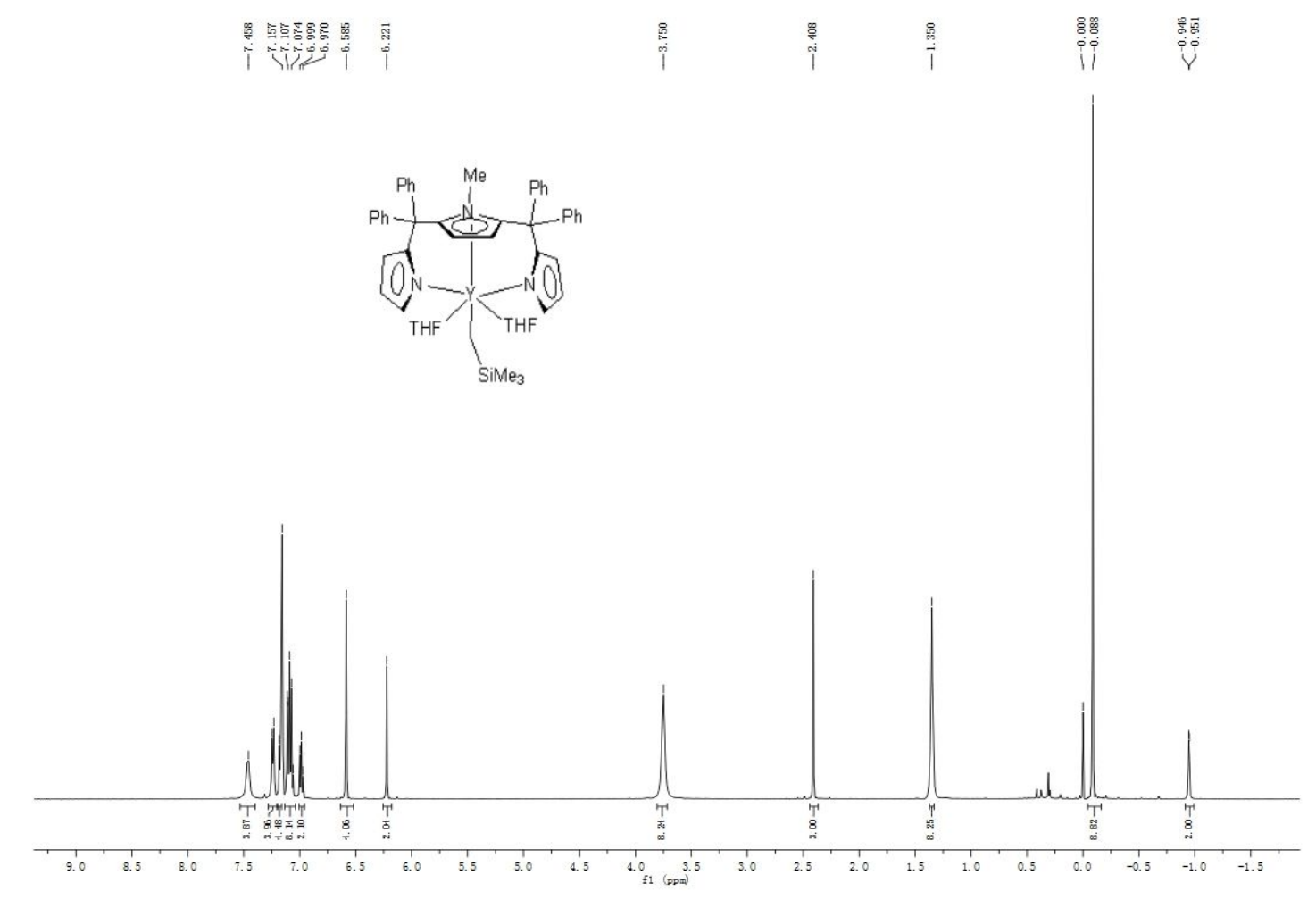

Figure S3. ${ }^{1} \mathrm{H}$ NMR $\left(500 \mathrm{MHz}, \mathrm{C}_{6} \mathrm{D}_{6}\right)$ spectrum of complex 1a

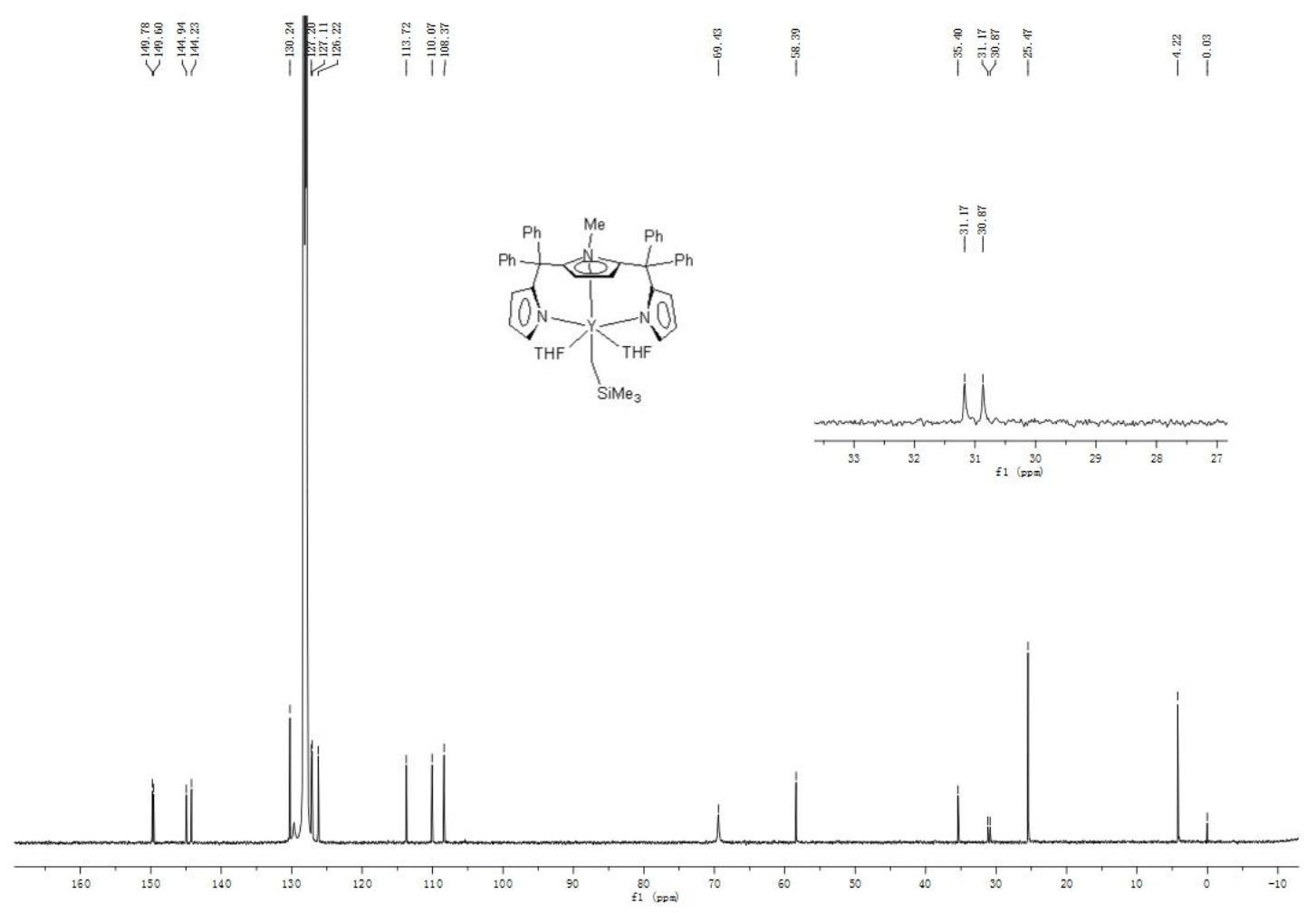

Figure S4. ${ }^{13} \mathrm{C}$ NMR $\left(125 \mathrm{MHz}, \mathrm{C}_{6} \mathrm{D}_{6}\right)$ spectrum of complex $1 \mathbf{a}$ 

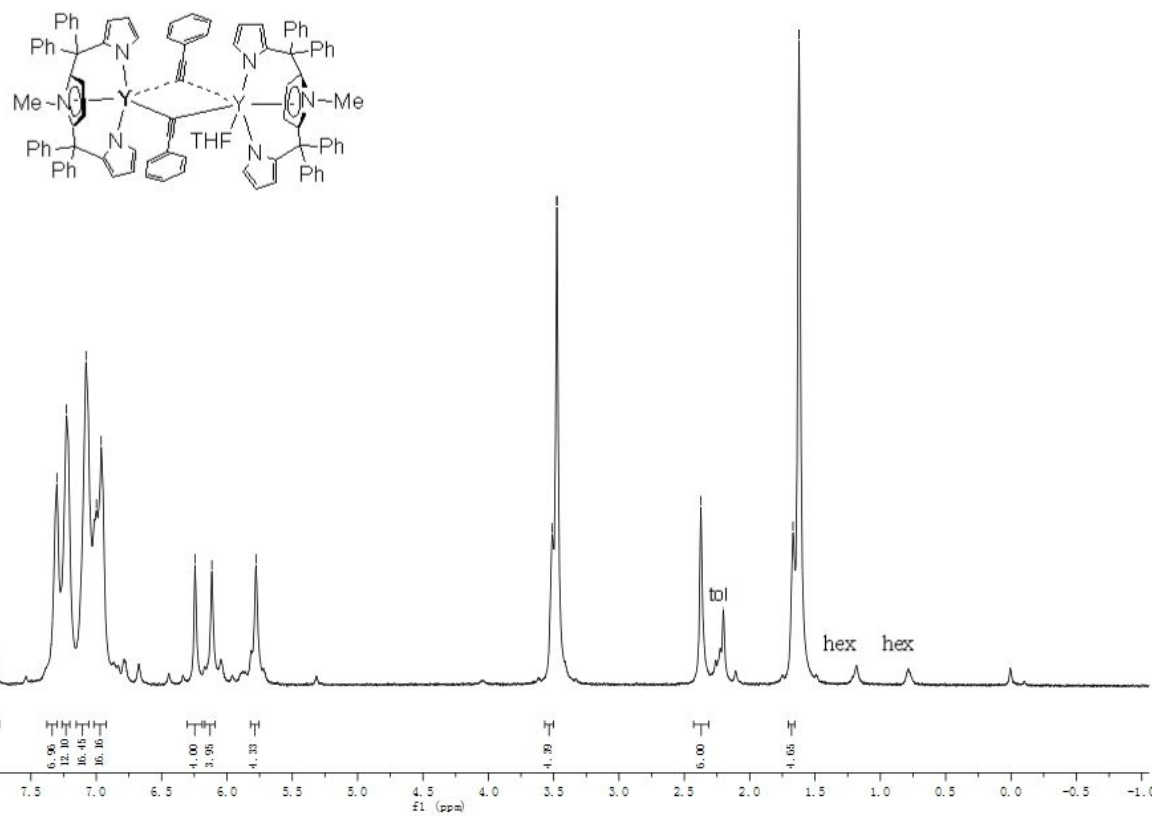

Figure S5. ${ }^{1} \mathrm{H}$ NMR $\left(500 \mathrm{MHz}, \mathrm{THF}-d_{8}\right)$ spectrum of complex 5a

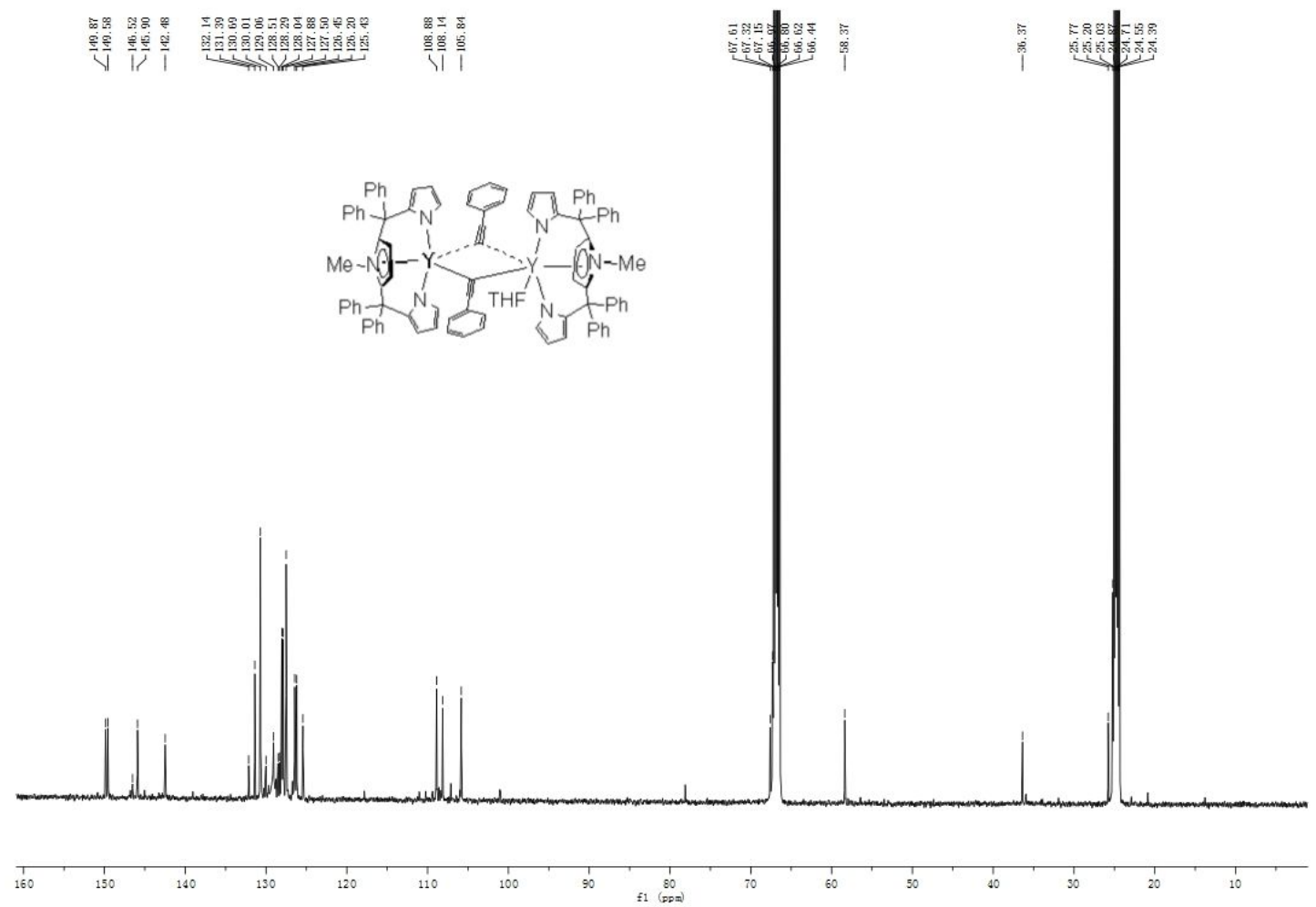

Figure S6. ${ }^{13} \mathrm{C}$ NMR $\left(125 \mathrm{MHz}, \mathrm{THF}-d_{8}\right)$ spectrum of complex 5a 

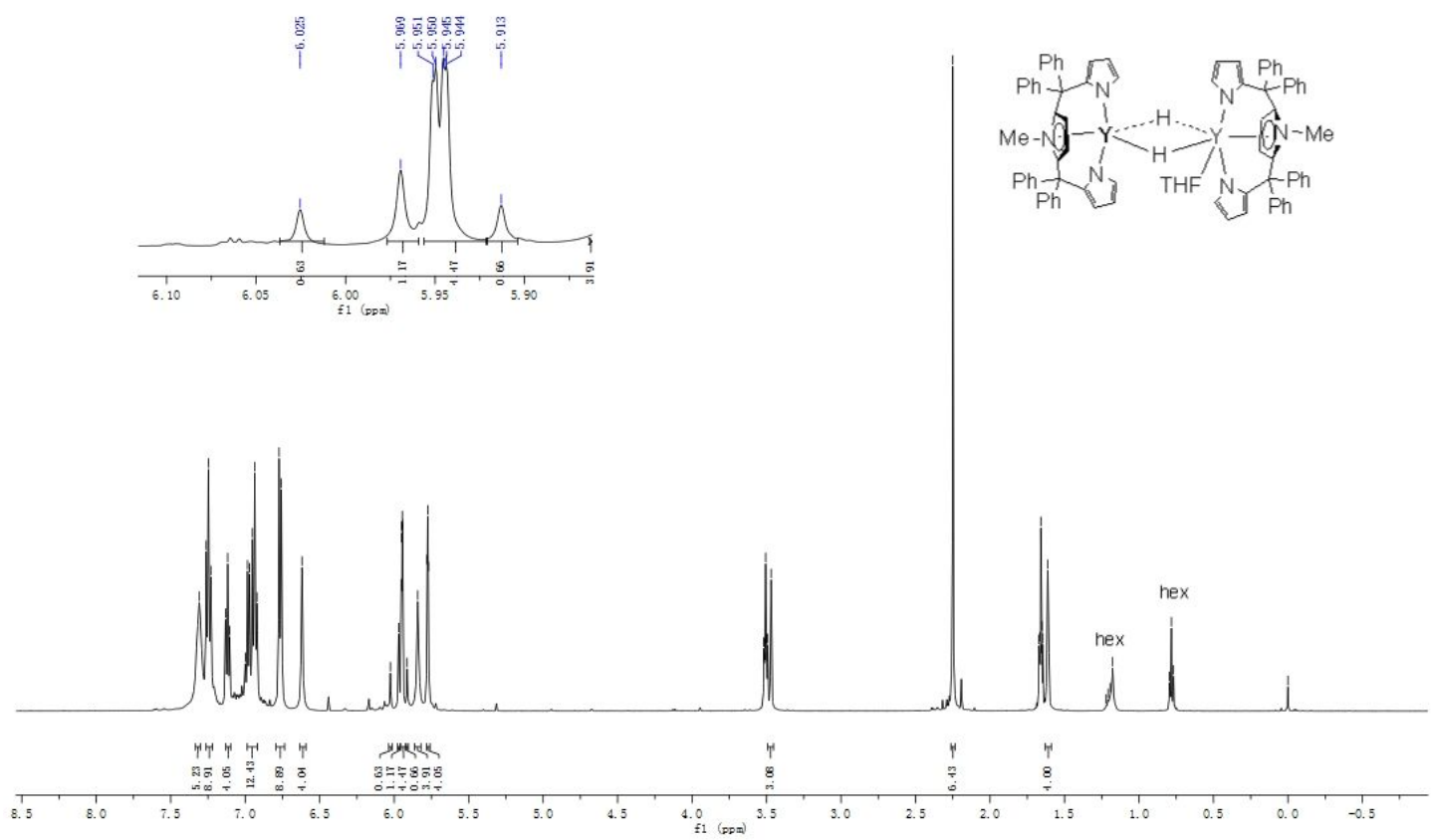

Figure S7. ${ }^{1} \mathrm{H}$ NMR $\left(500 \mathrm{MHz}, \mathrm{THF}-d_{8}\right)$ spectrum of complex $6 \mathbf{a}$

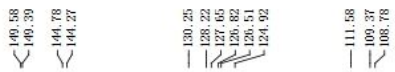

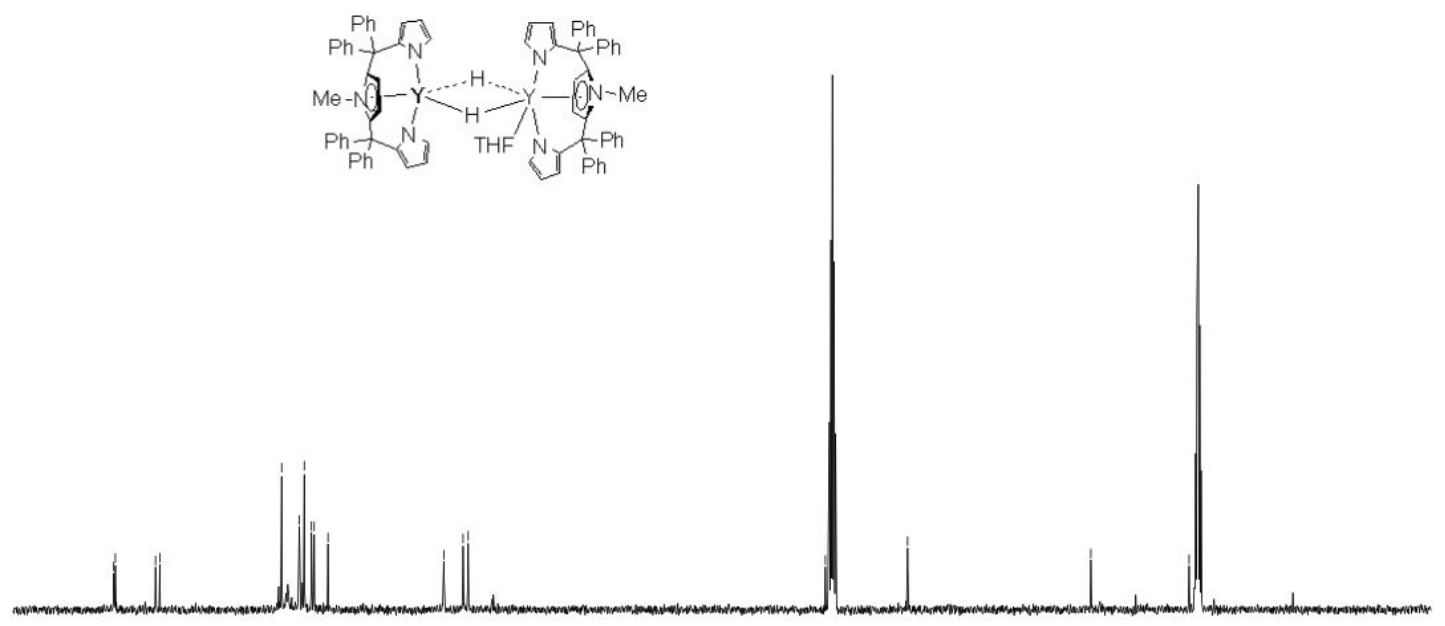

Figure S8. ${ }^{13} \mathrm{C}$ NMR $\left(125 \mathrm{MHz}, \mathrm{THF}-d_{8}\right)$ spectrum of complex $6 \mathbf{a}$ 

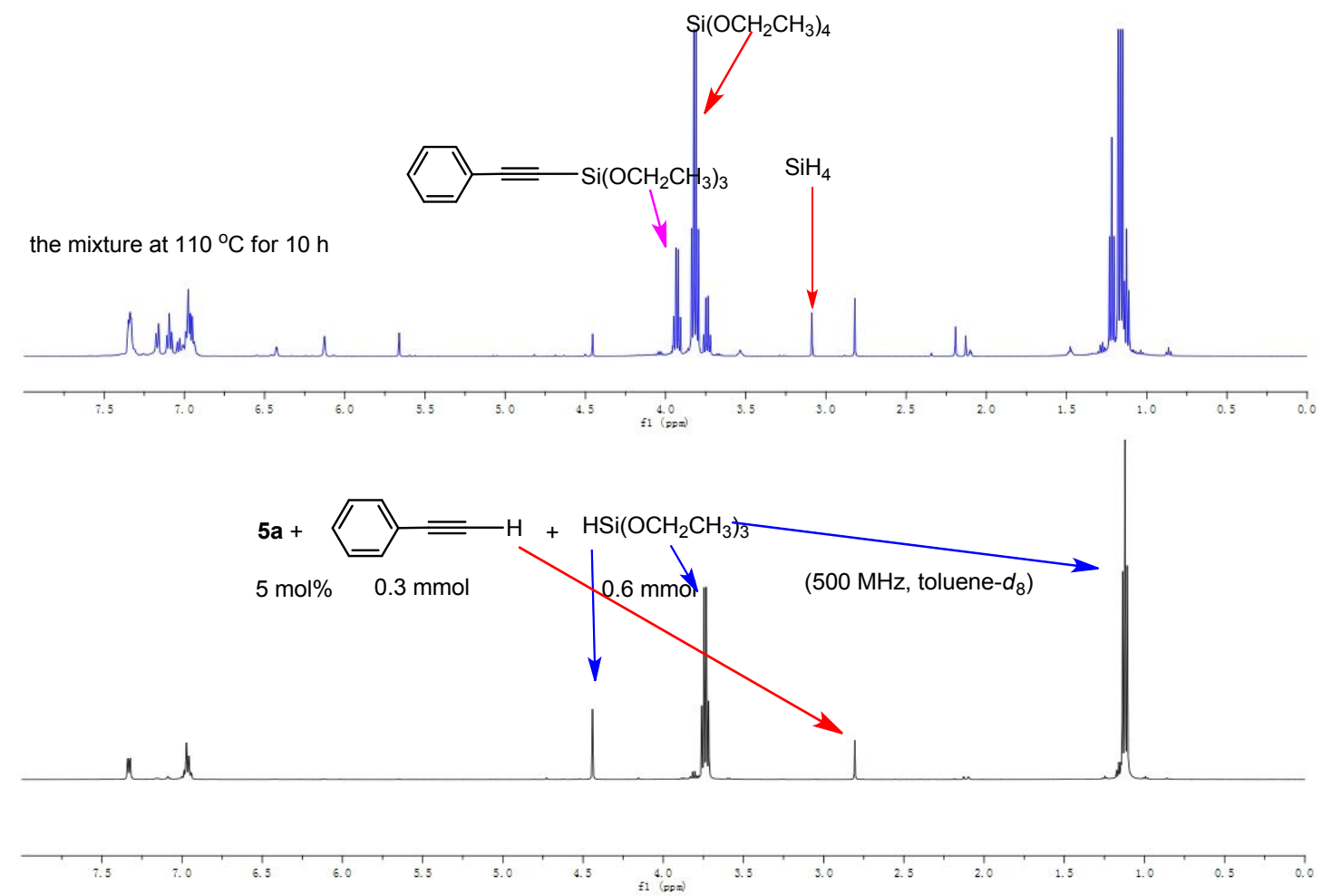

Figure S9. ${ }^{1} \mathrm{H}$ NMR monitoring the catalytic reaction of phenylacetylene and $\mathrm{HSi}(\mathrm{OEt})_{3}$ by $\mathbf{5 a}\left(500 \mathrm{MHz}\right.$, toluene- $\left.d_{8}\right)$

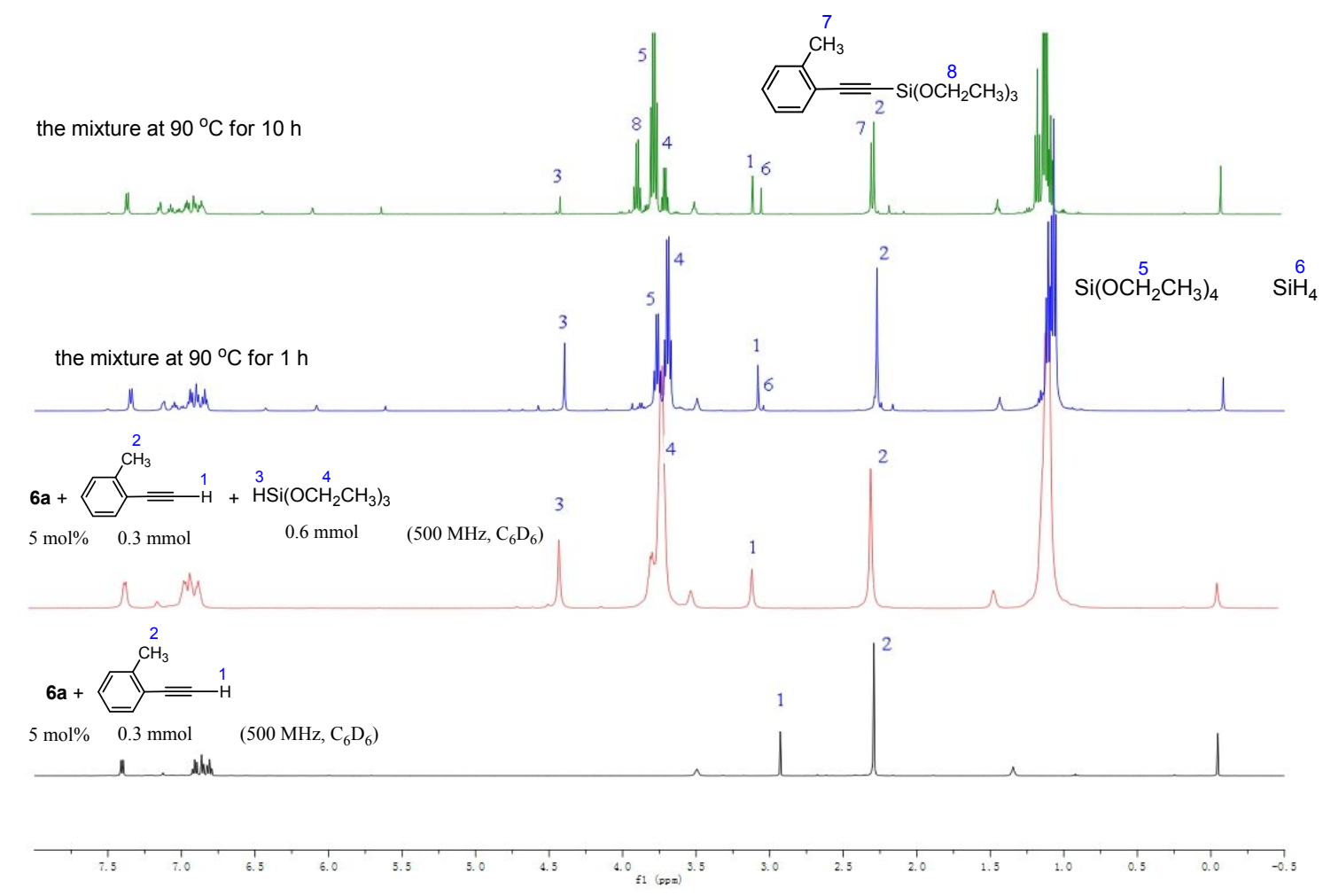

Figure S10. ${ }^{1} \mathrm{H}$ NMR monitoring the catalytic reaction of $o$-methylphenylacetylene and $\mathrm{HSi}(\mathrm{OEt})_{3}$ by $\mathbf{6 a}\left(500 \mathrm{MHz}, \mathrm{C}_{6} \mathrm{D}_{6}\right)$ 
${ }^{1} \mathrm{H}$ NMR and ${ }^{13} \mathrm{C}$ NMR spectra of compounds of $4 \mathbf{a a}-4 \mathbf{s d}$
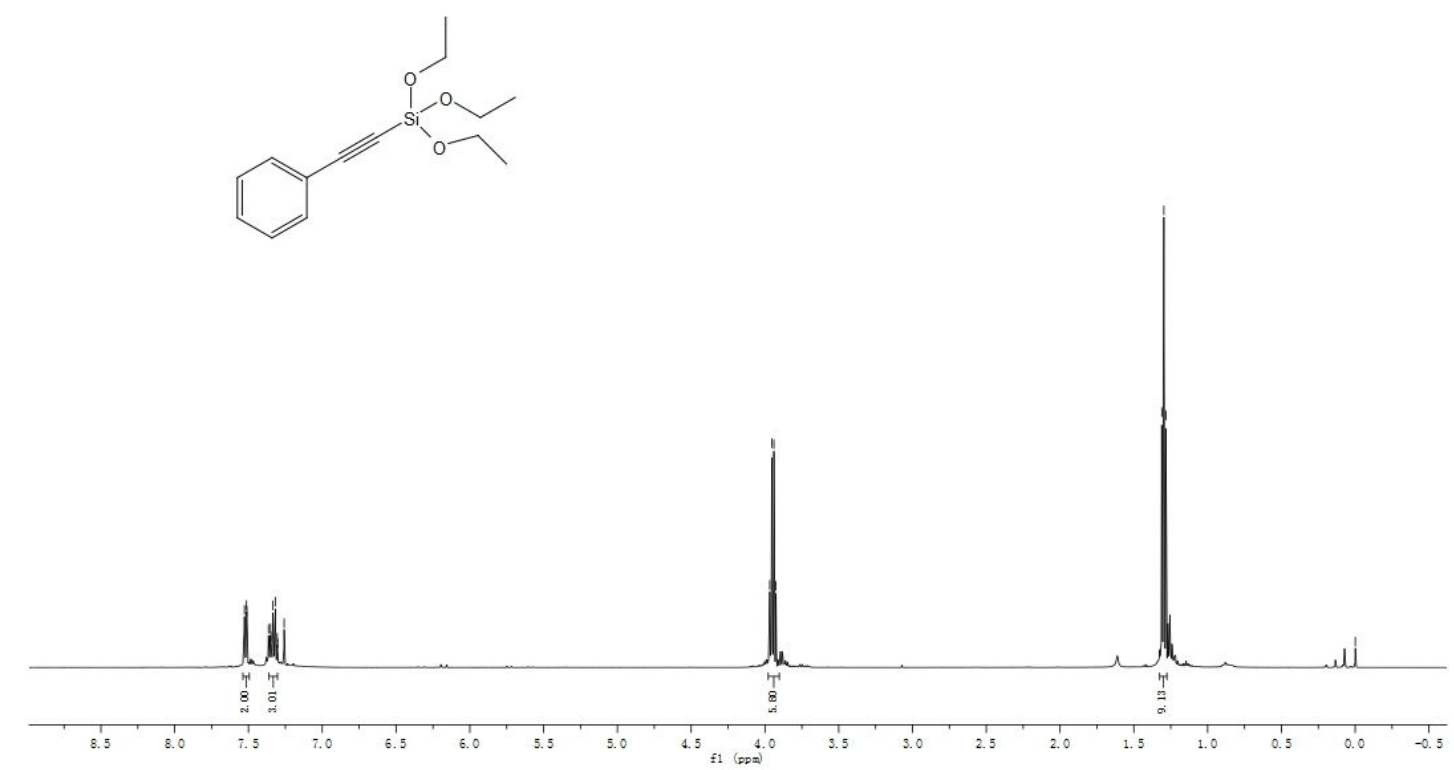

${ }^{1} \mathrm{H}$ NMR (500 MHz, $\mathrm{CDCl}_{3}$ ) spectrum of $\mathbf{4 a a}$
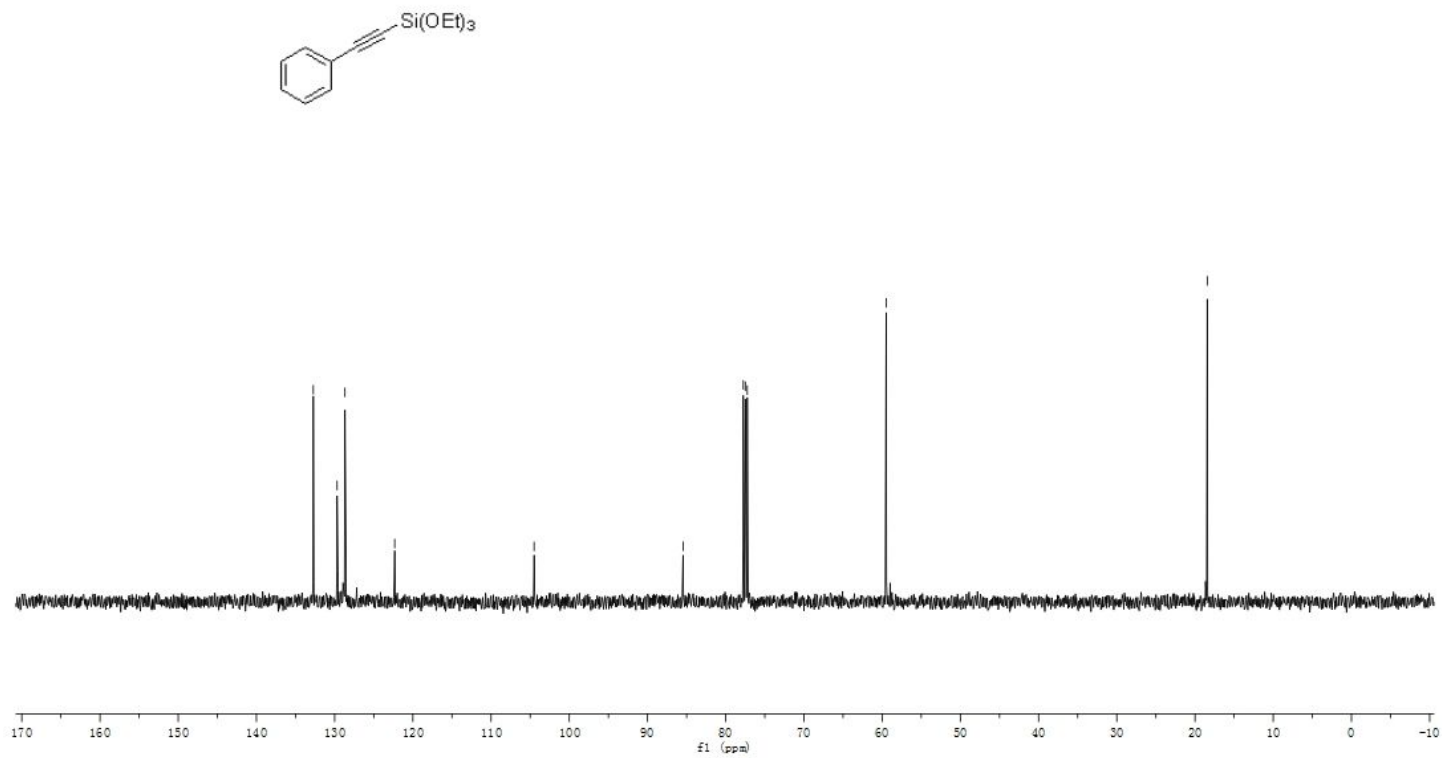

${ }^{13} \mathrm{C} \mathrm{NMR}\left(125 \mathrm{MHz}, \mathrm{CDCl}_{3}\right)$ spectrum of $4 \mathbf{a a}$ 


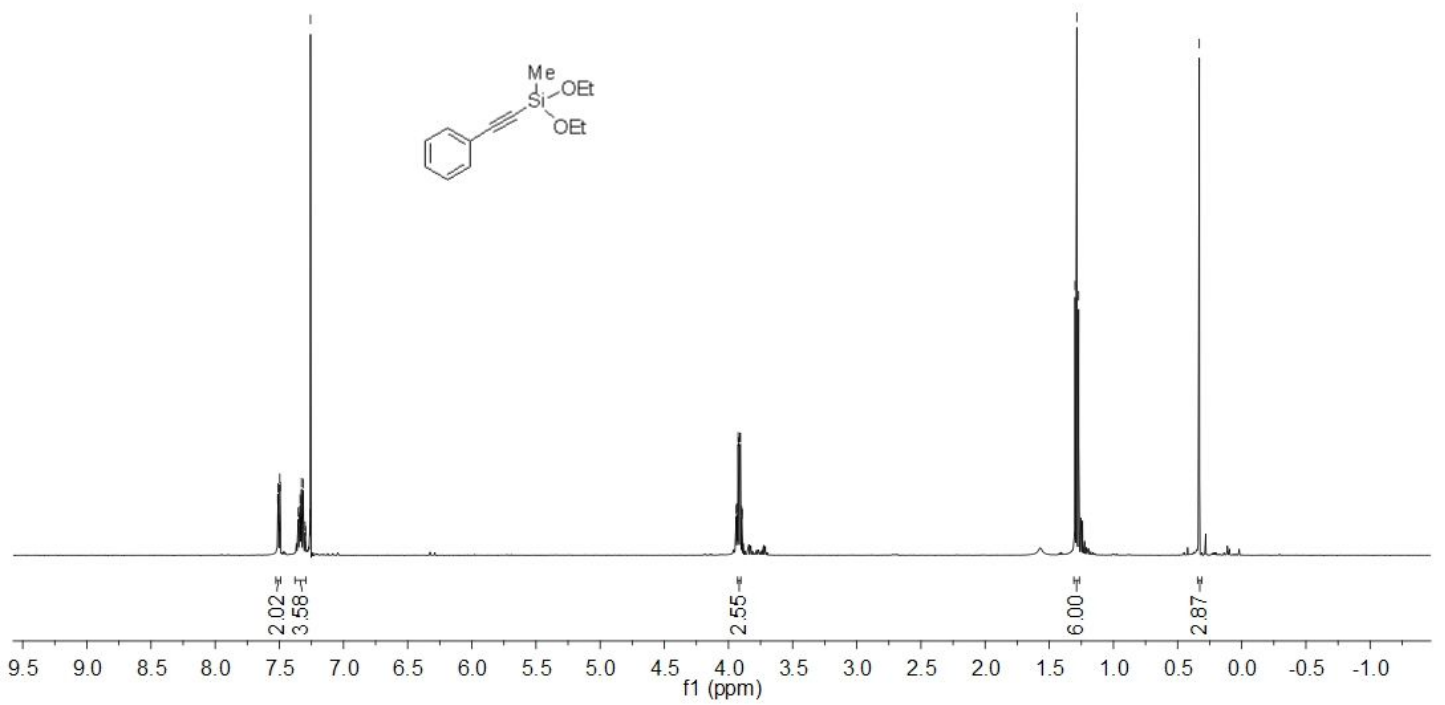

${ }^{1} \mathrm{H}$ NMR $\left(500 \mathrm{MHz}, \mathrm{CDCl}_{3}\right)$ spectrum of $\mathbf{4 a b}$
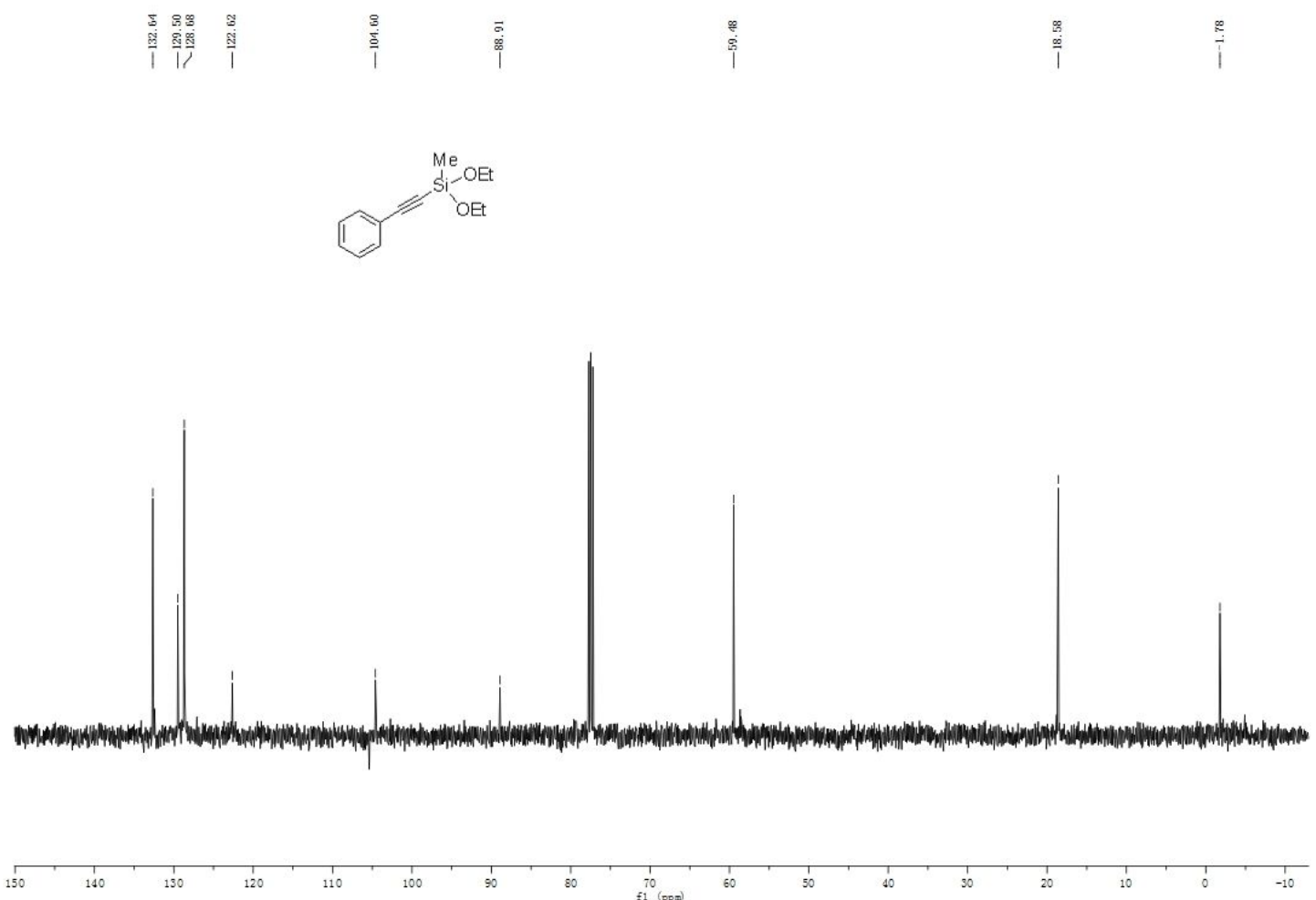

${ }^{13} \mathrm{C}$ NMR $\left(125 \mathrm{MHz}, \mathrm{CDCl}_{3}\right.$ ) spectrum of $4 \mathbf{a b}$ 


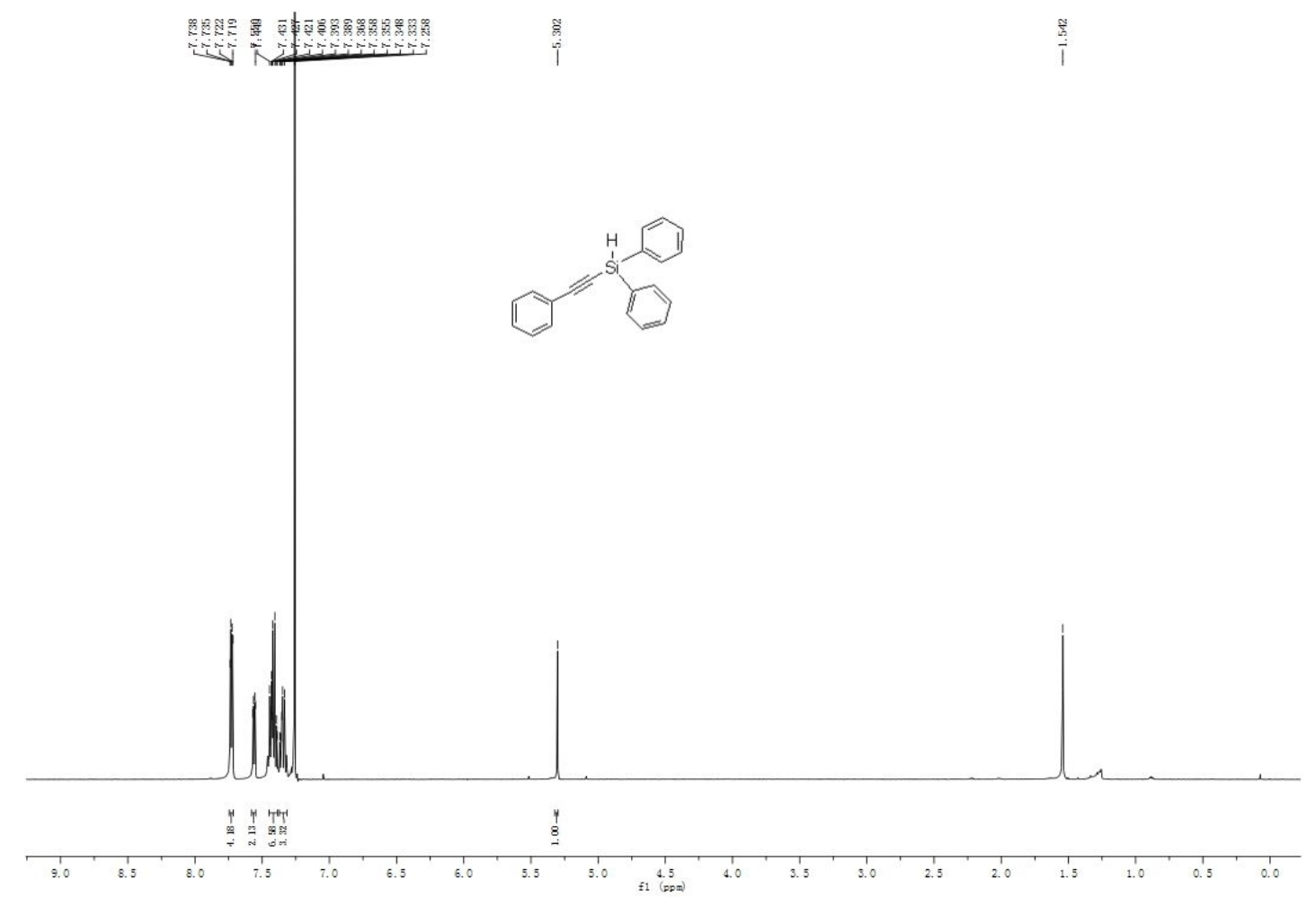

${ }^{1} \mathrm{H}$ NMR $\left(500 \mathrm{MHz}, \mathrm{CDCl}_{3}\right)$ spectrum of $4 \mathbf{a f}$

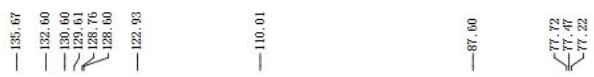
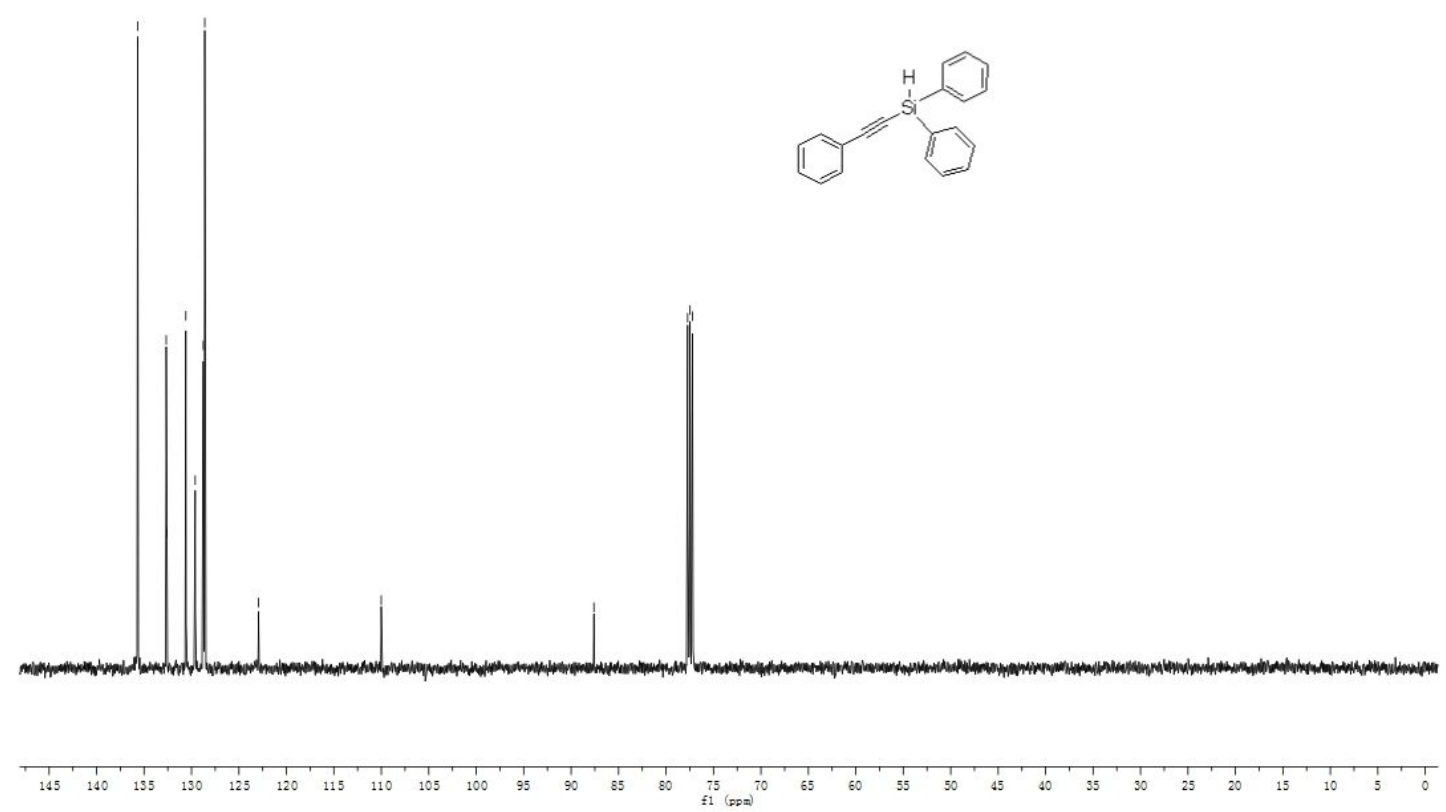

${ }^{13} \mathrm{C}$ NMR $\left(125 \mathrm{MHz}, \mathrm{CDCl}_{3}\right)$ spectrum of $\mathbf{4 a f}$ 


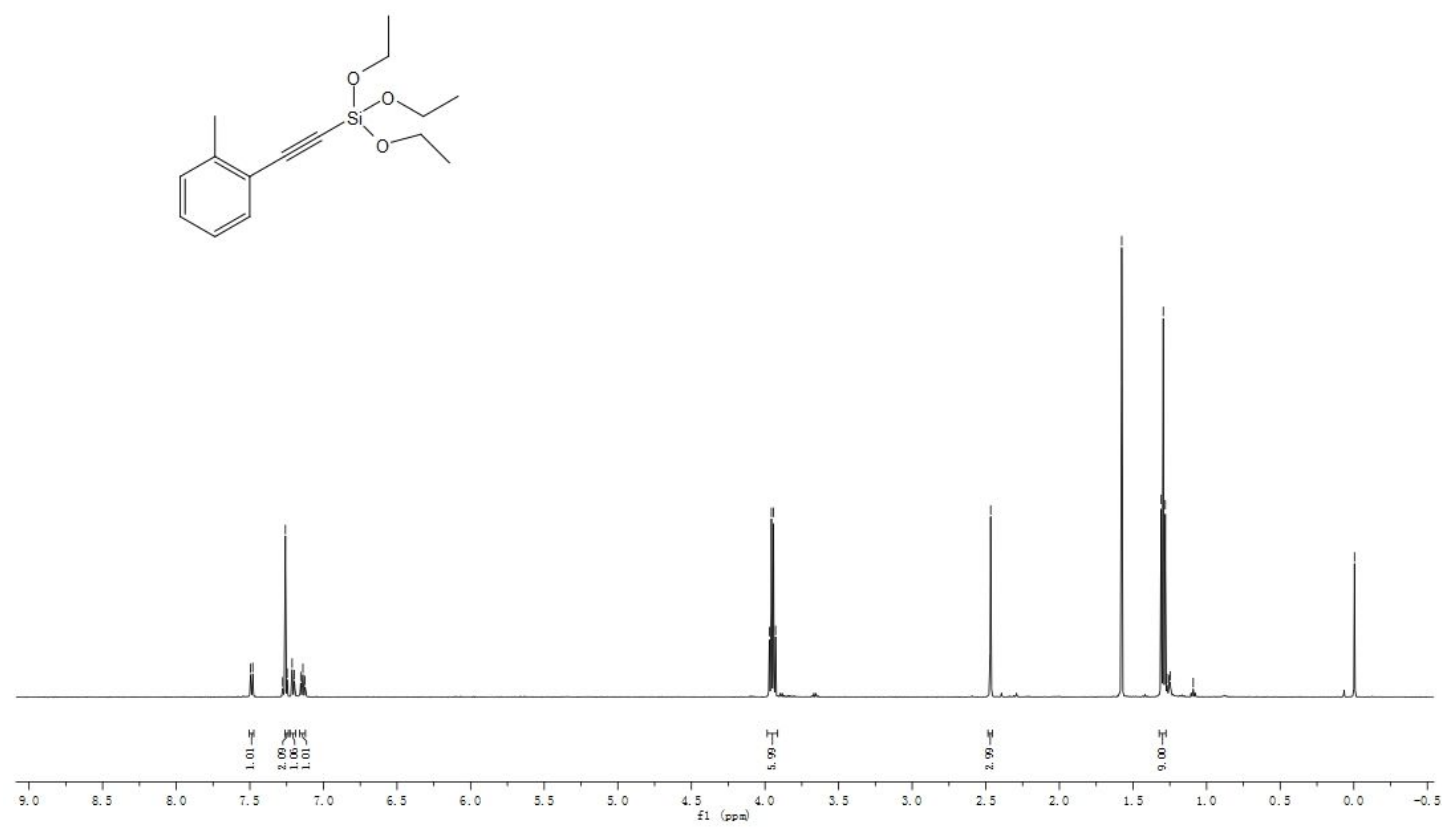

${ }^{1} \mathrm{H}$ NMR $\left(500 \mathrm{MHz}, \mathrm{CDCl}_{3}\right)$ spectrum of $\mathbf{4} \mathbf{b a}$

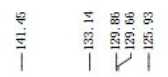
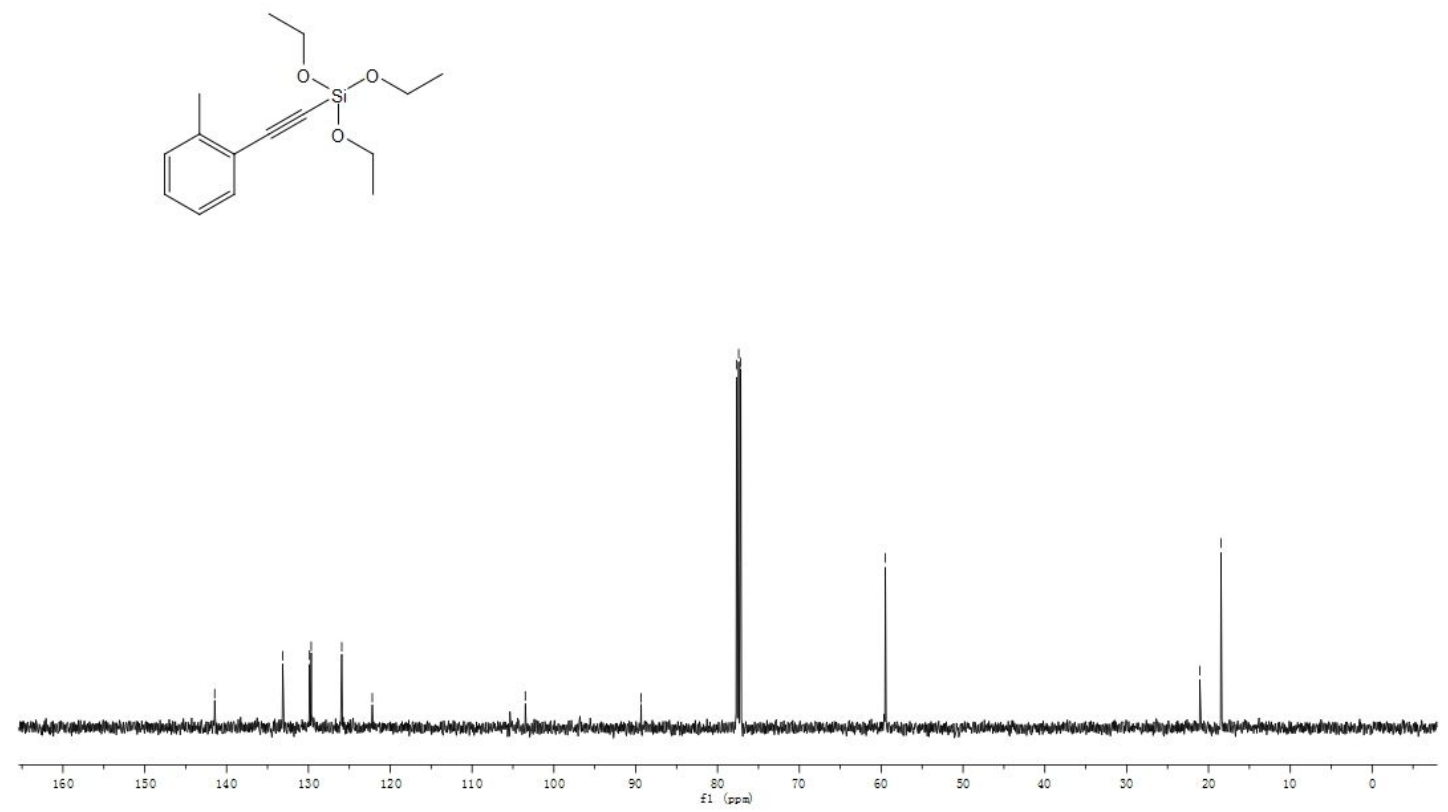

${ }^{13} \mathrm{C} \mathrm{NMR}\left(125 \mathrm{MHz}, \mathrm{CDCl}_{3}\right)$ spectrum of $\mathbf{4} \mathbf{b a}$ 


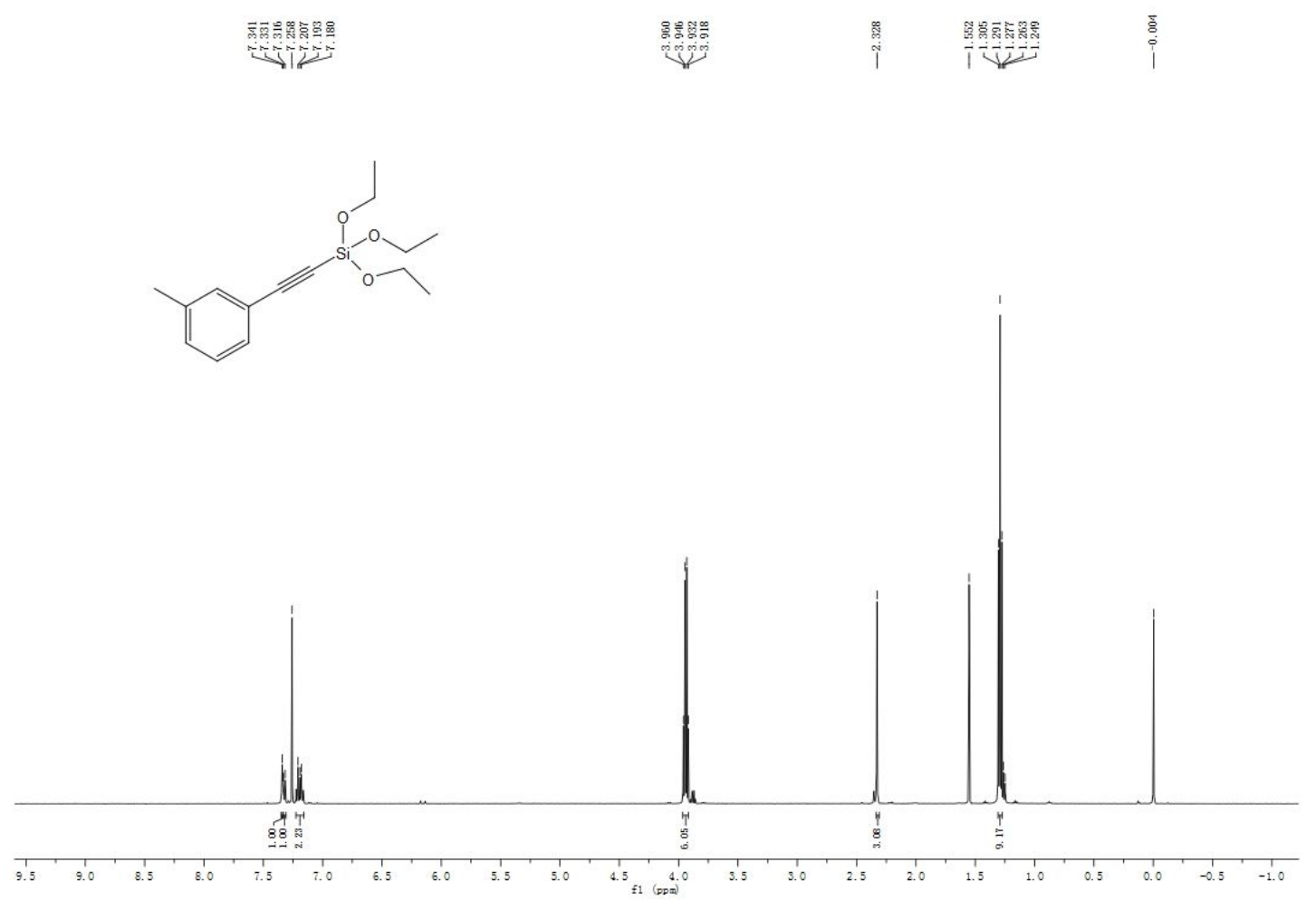

${ }^{1} \mathrm{H}$ NMR $\left(500 \mathrm{MHz}, \mathrm{CDCl}_{3}\right)$ spectrum of $\mathbf{4} \mathbf{c a}$
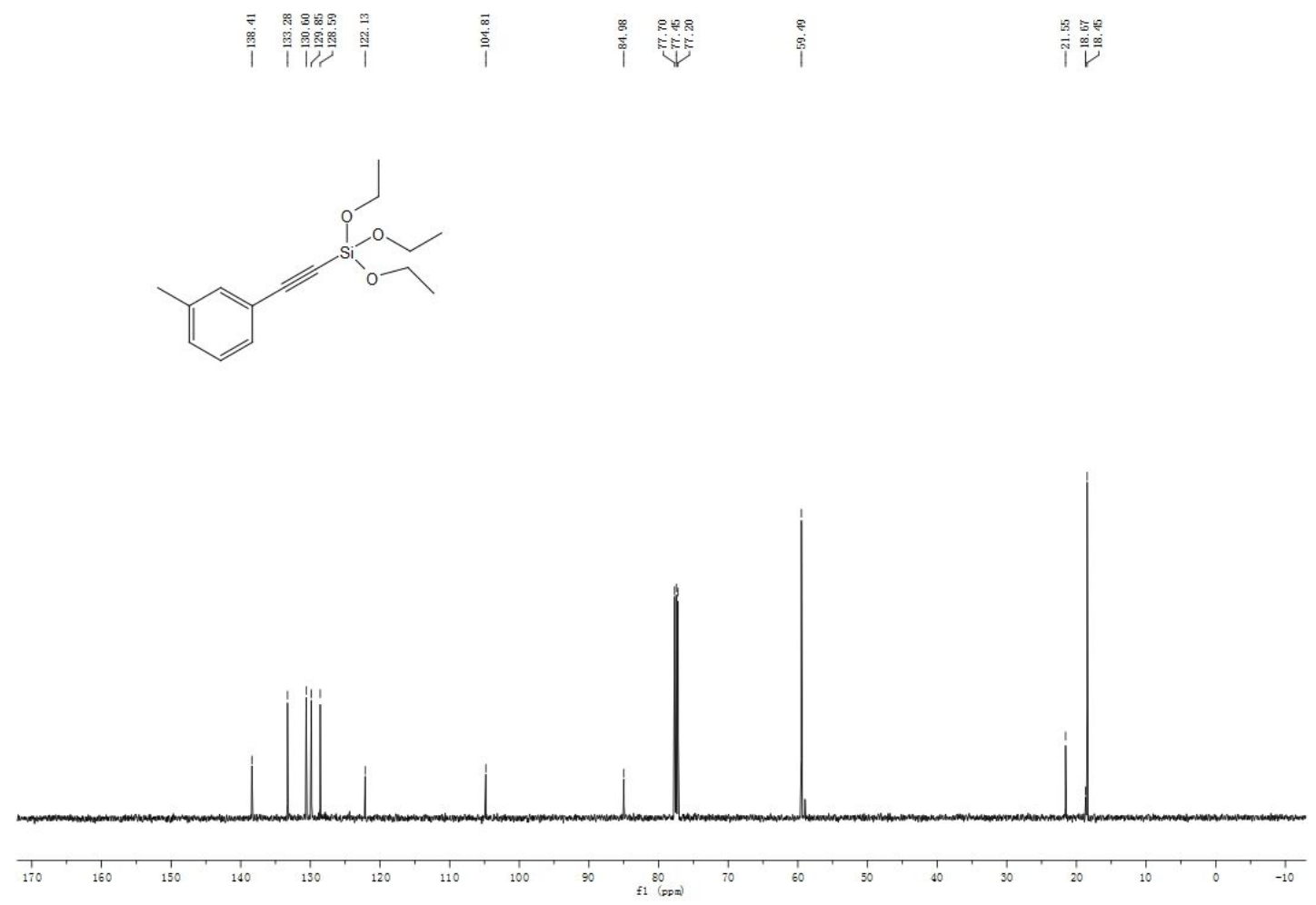

${ }^{13} \mathrm{C} \mathrm{NMR}\left(125 \mathrm{MHz}, \mathrm{CDCl}_{3}\right)$ spectrum of $\mathbf{4} \mathbf{c a}$ 


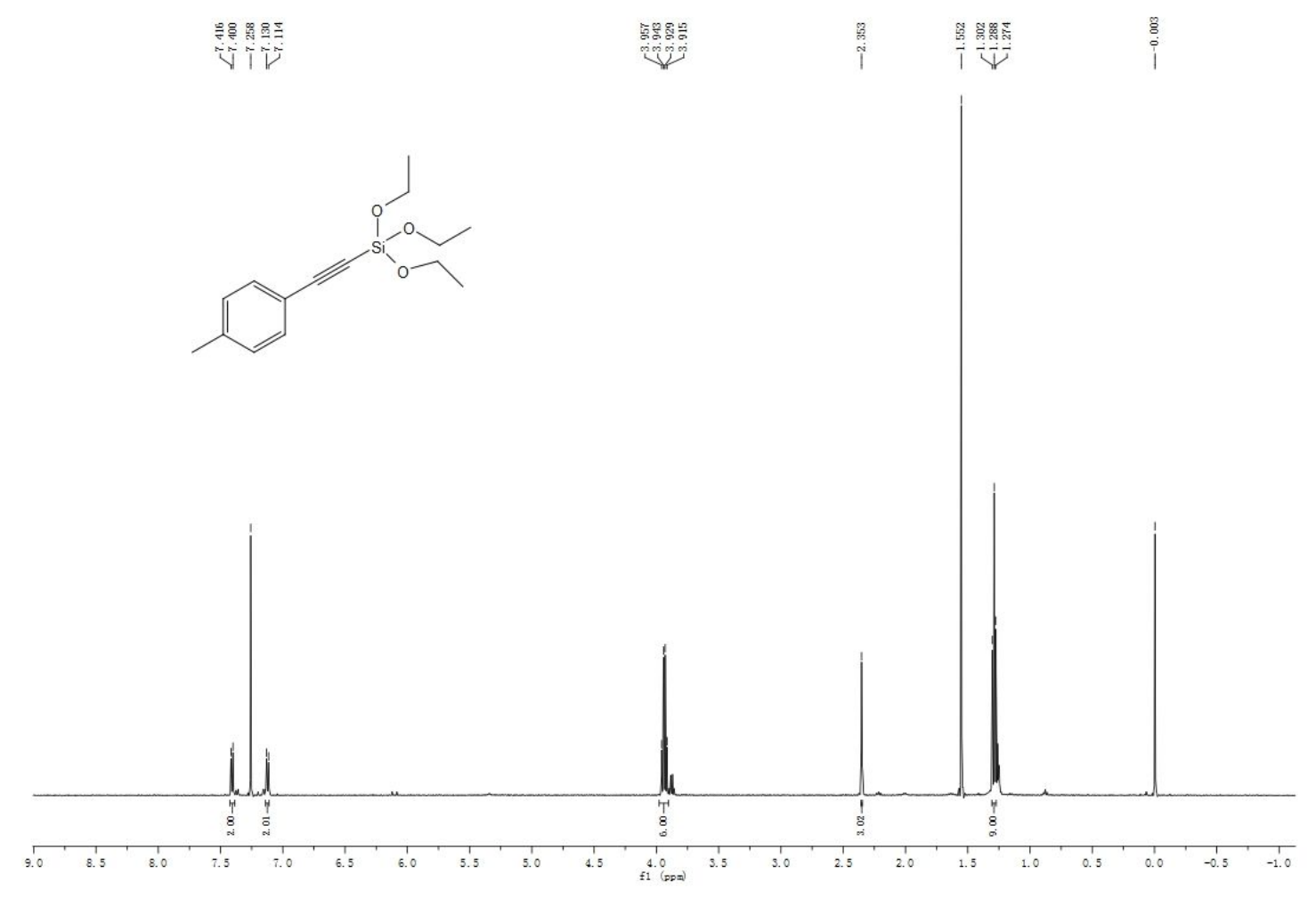

${ }^{1} \mathrm{H}$ NMR (500 MHz, $\mathrm{CDCl}_{3}$ ) spectrum of $\mathbf{4 d a}$
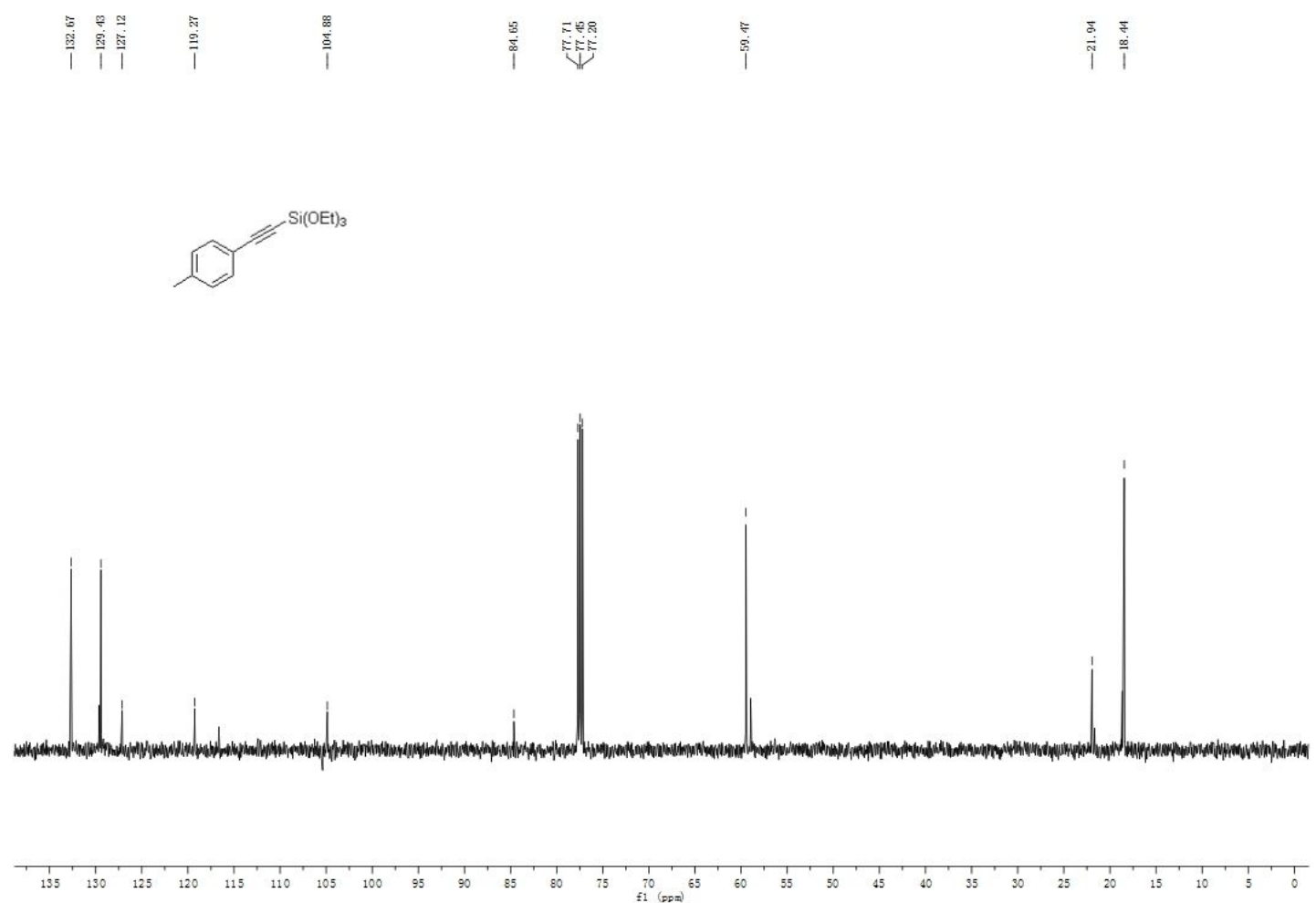

${ }^{13} \mathrm{C}$ NMR $\left(125 \mathrm{MHz}, \mathrm{CDCl}_{3}\right)$ spectrum of $4 \mathbf{d a}$ 


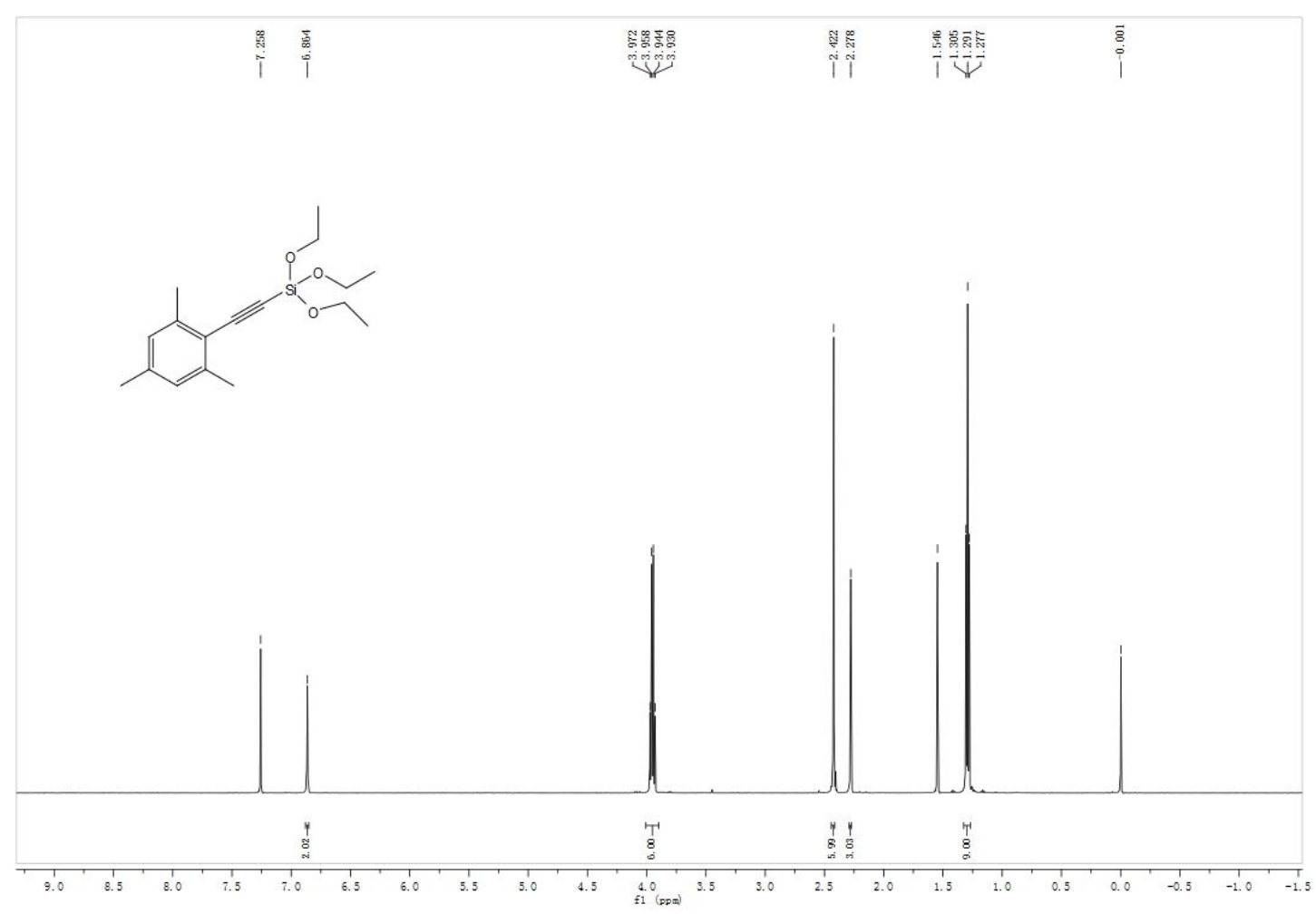

${ }^{1} \mathrm{H}$ NMR $\left(500 \mathrm{MHz}, \mathrm{CDCl}_{3}\right)$ spectrum of $4 \mathbf{e a}$
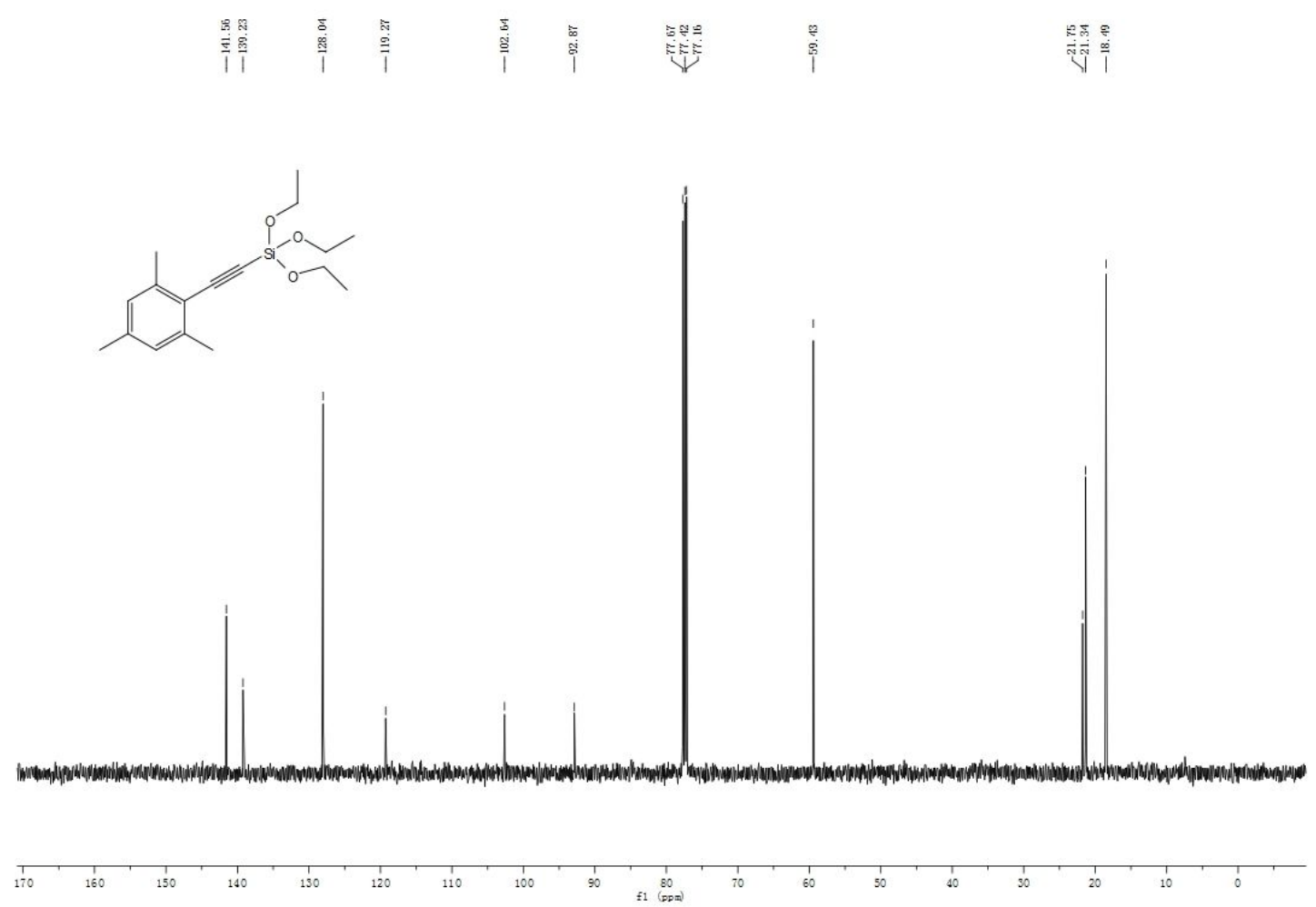

${ }^{13} \mathrm{C}$ NMR $\left(125 \mathrm{MHz}, \mathrm{CDCl}_{3}\right)$ spectrum of $4 \mathbf{e a}$ 


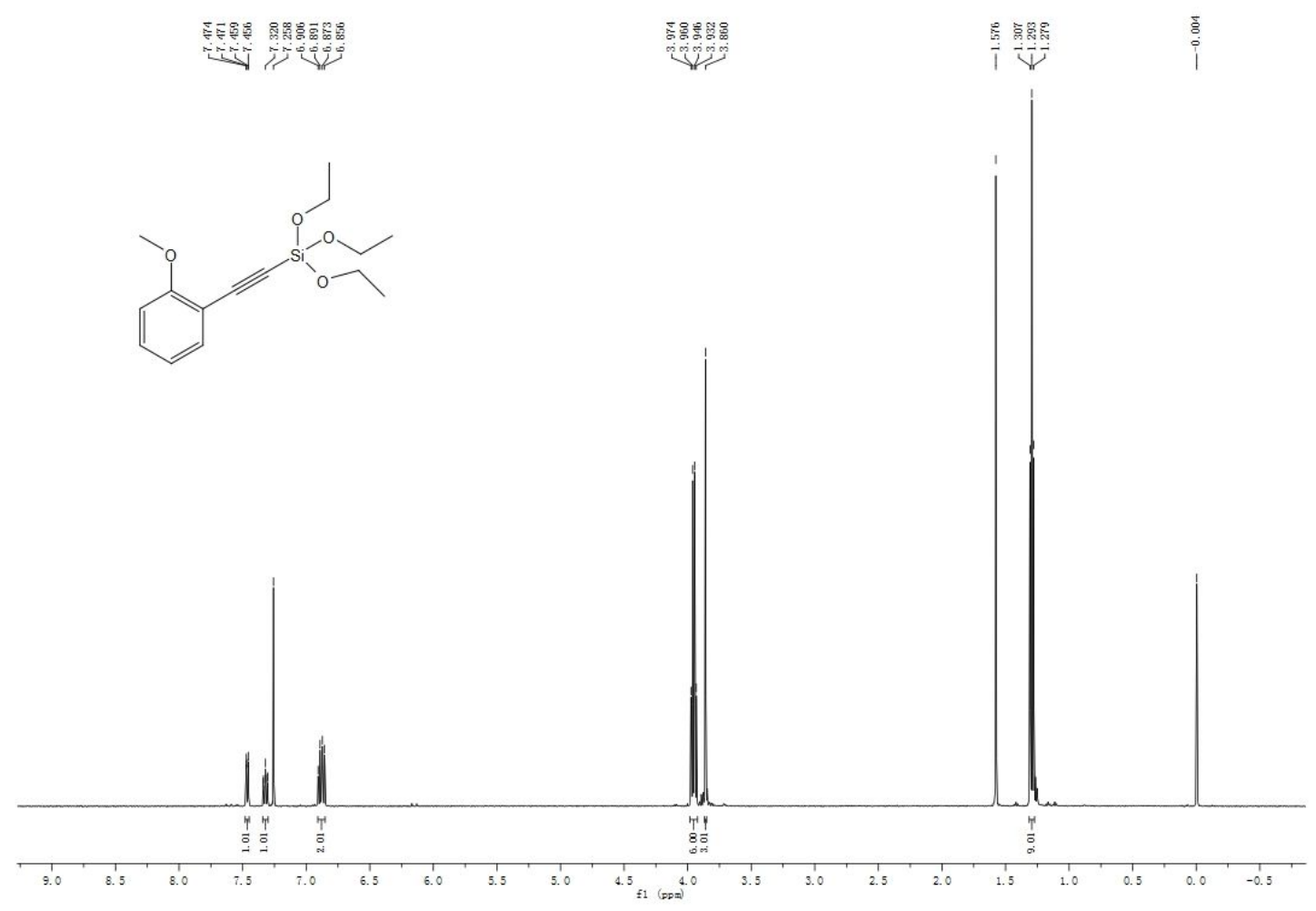

${ }^{1} \mathrm{H}$ NMR $\left(500 \mathrm{MHz}, \mathrm{CDCl}_{3}\right.$ ) spectrum of $\mathbf{4 f a}$
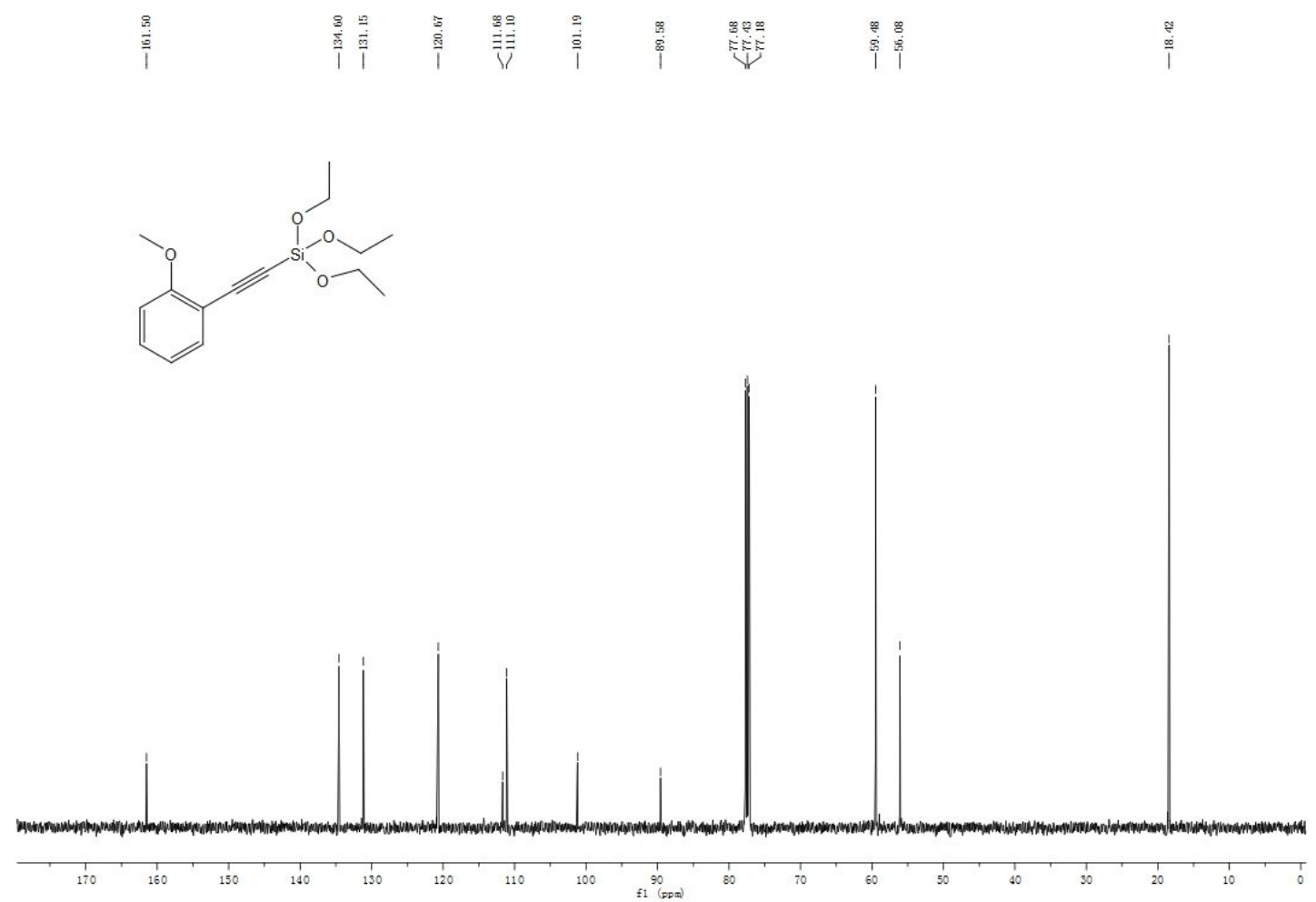

${ }^{13} \mathrm{C}$ NMR $\left(125 \mathrm{MHz}, \mathrm{CDCl}_{3}\right)$ spectrum of $\mathbf{4 f a}$ 


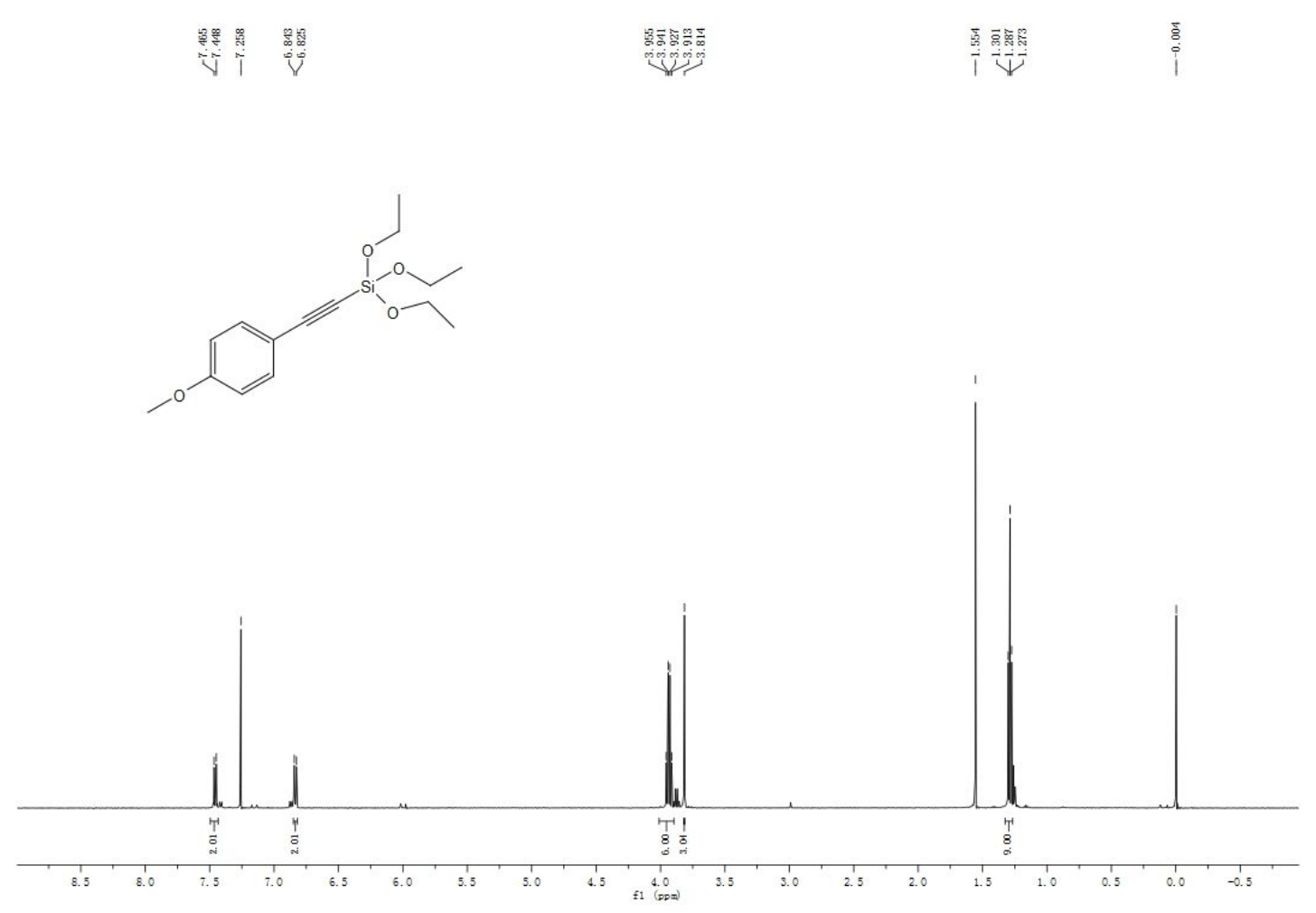

${ }^{1} \mathrm{H}$ NMR $\left(500 \mathrm{MHz}, \mathrm{CDCl}_{3}\right)$ spectrum of $\mathbf{4 g a}$

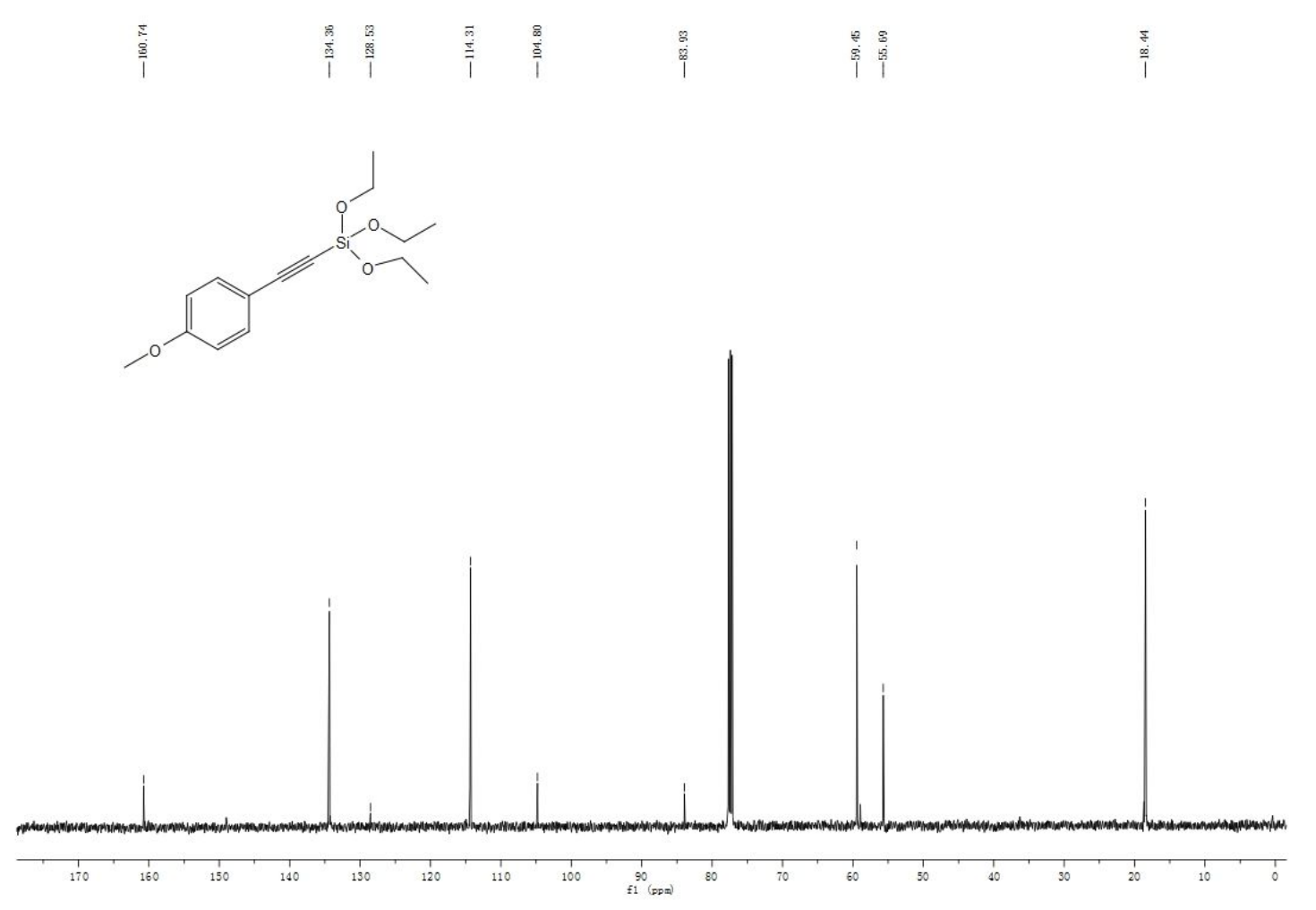

${ }^{13} \mathrm{C} \mathrm{NMR}\left(125 \mathrm{MHz}, \mathrm{CDCl}_{3}\right)$ spectrum of $4 \mathbf{g a}$ 


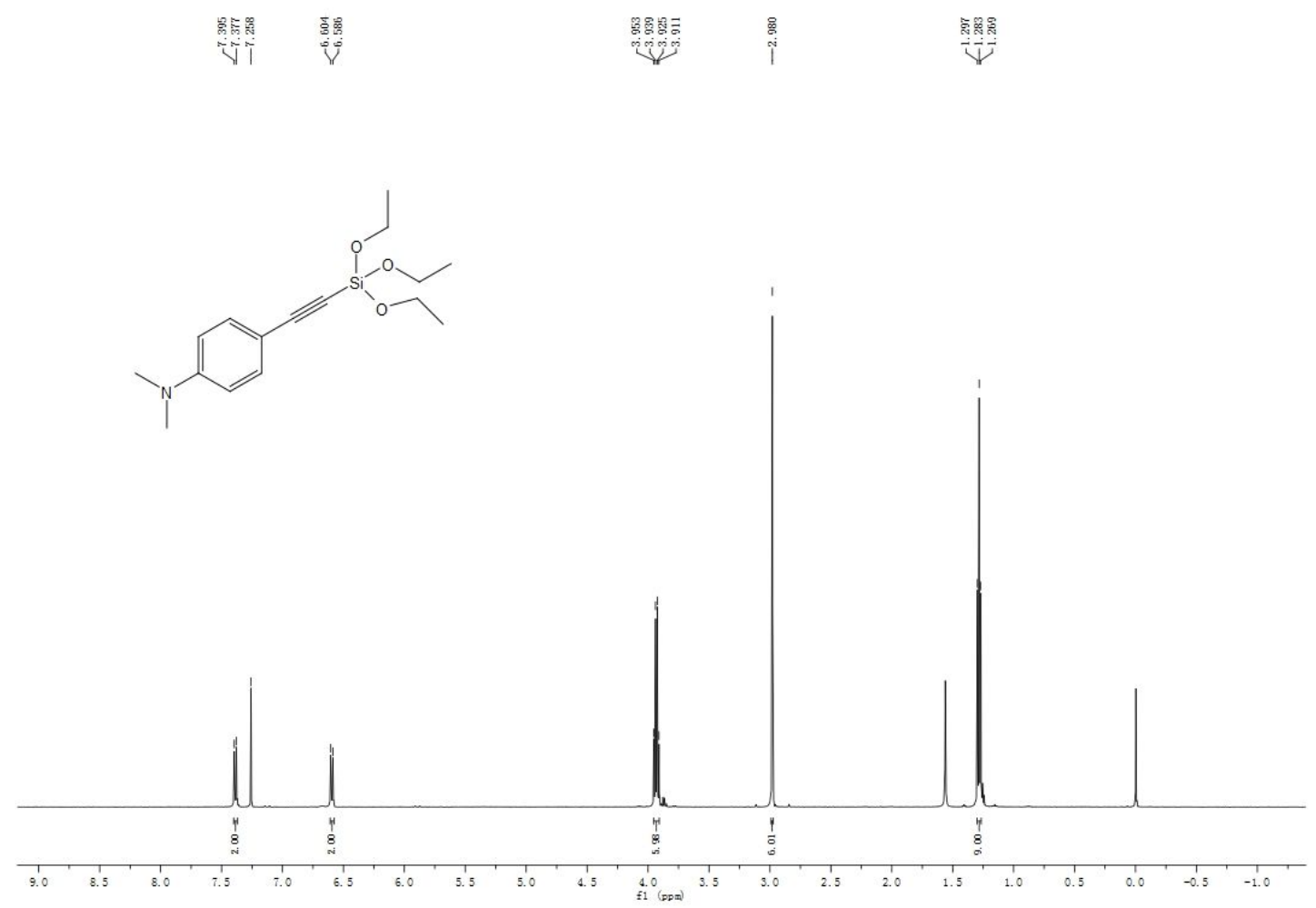

${ }^{1} \mathrm{H}$ NMR $\left(500 \mathrm{MHz}, \mathrm{CDCl}_{3}\right)$ spectrum of 4 ha
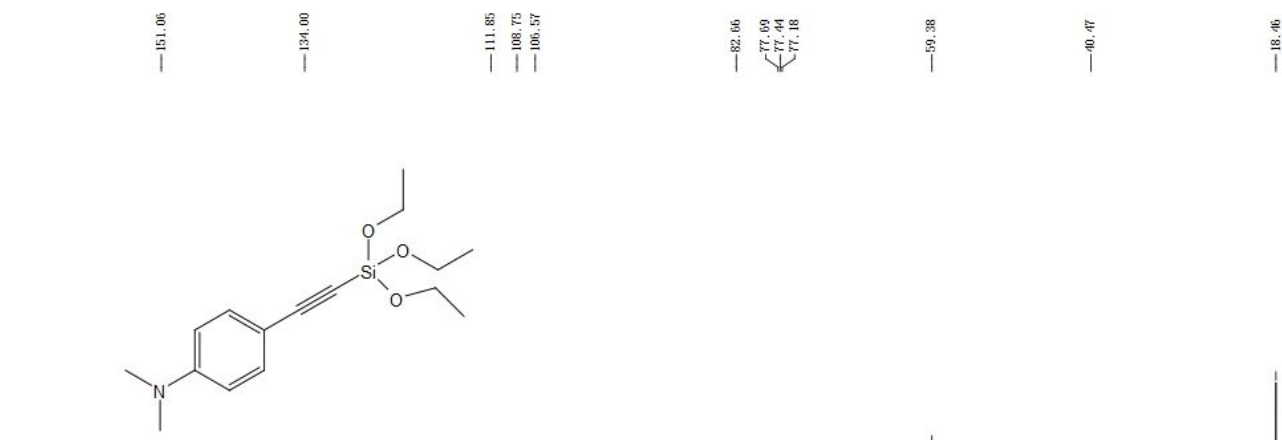

管

i (1)

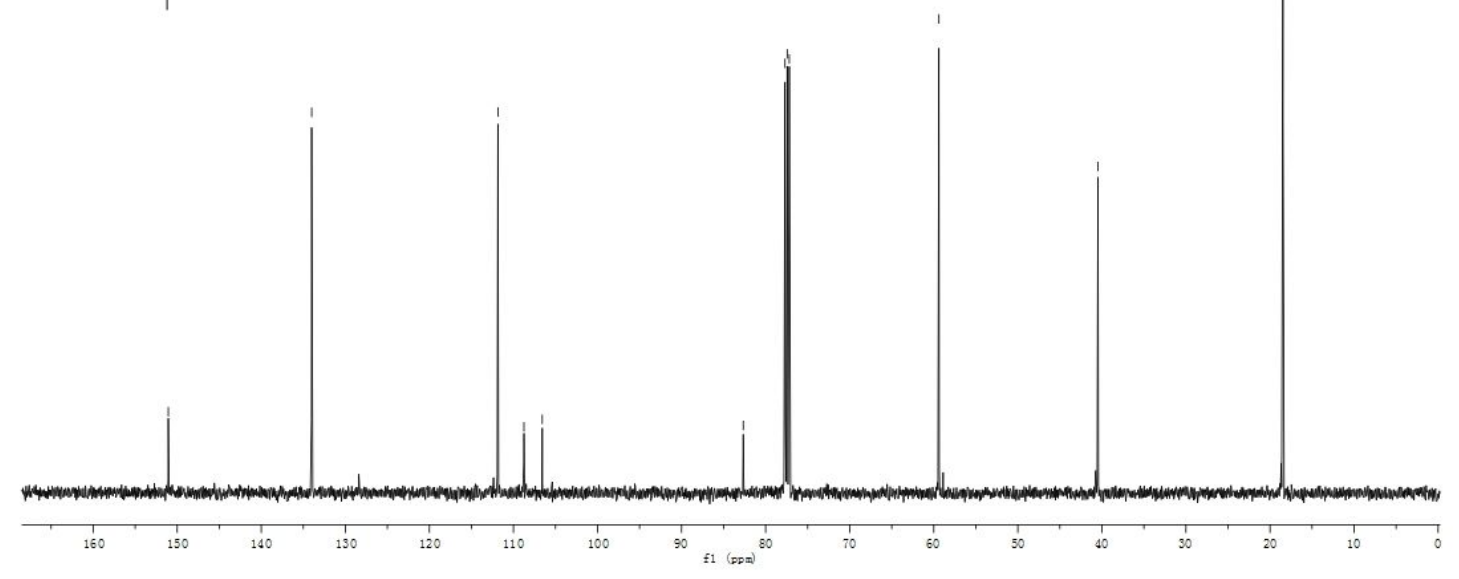

${ }^{13} \mathrm{C}$ NMR $\left(125 \mathrm{MHz}, \mathrm{CDCl}_{3}\right)$ spectrum of 4 ha 


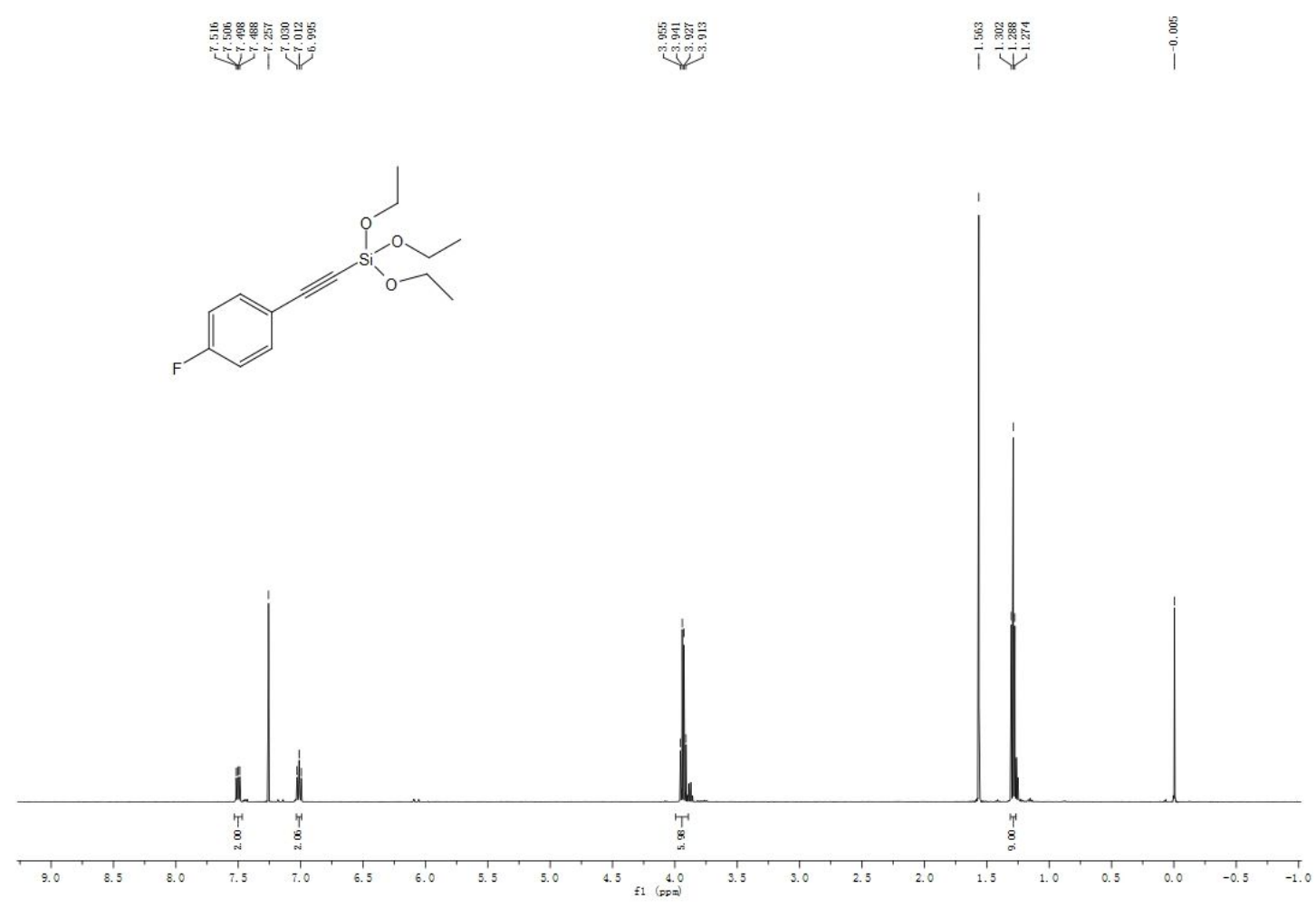

${ }^{1} \mathrm{H}$ NMR $\left(500 \mathrm{MHz}, \mathrm{CDCl}_{3}\right)$ spectrum of 4 ia

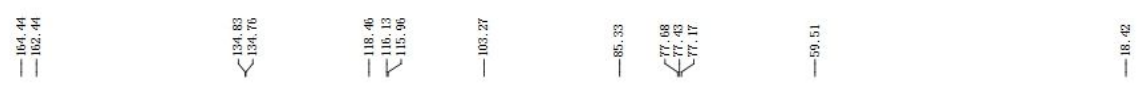
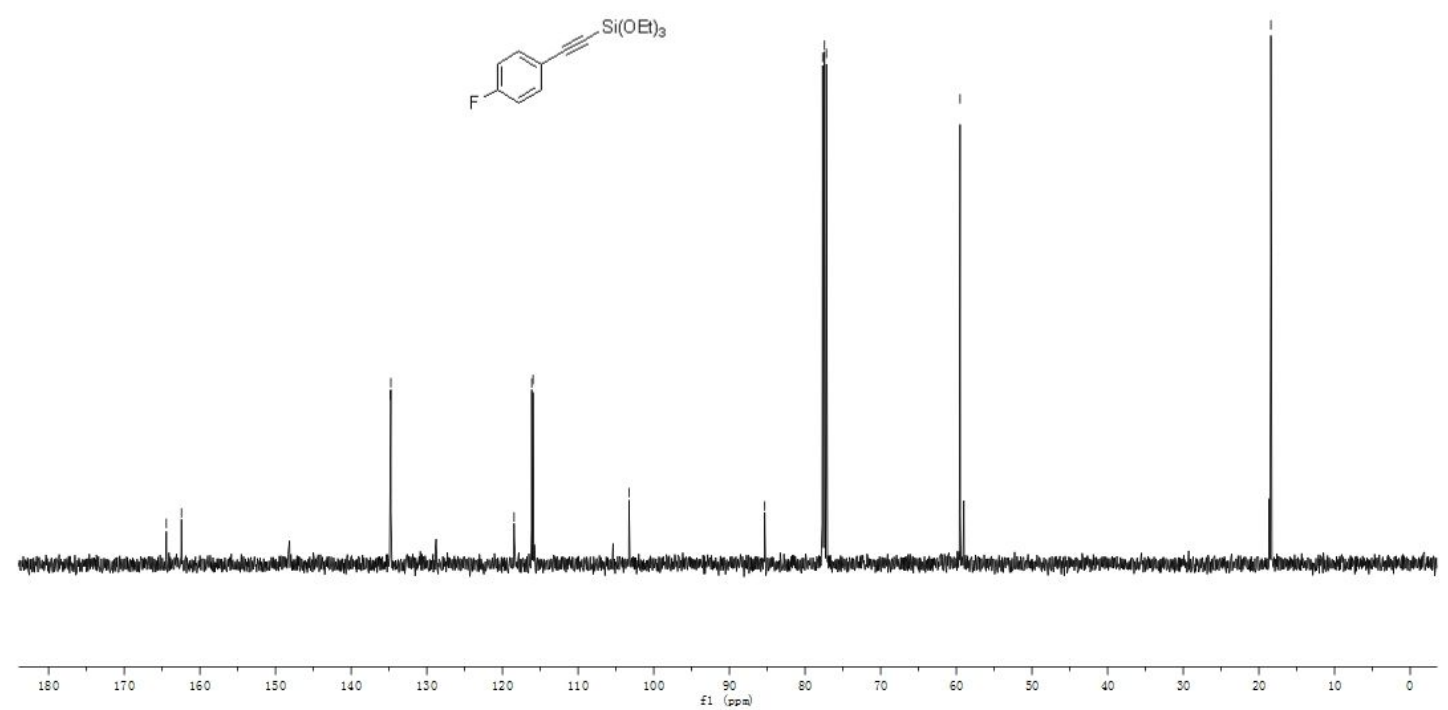

${ }^{13} \mathrm{C}$ NMR $\left(125 \mathrm{MHz}, \mathrm{CDCl}_{3}\right)$ spectrum of 4 ia 

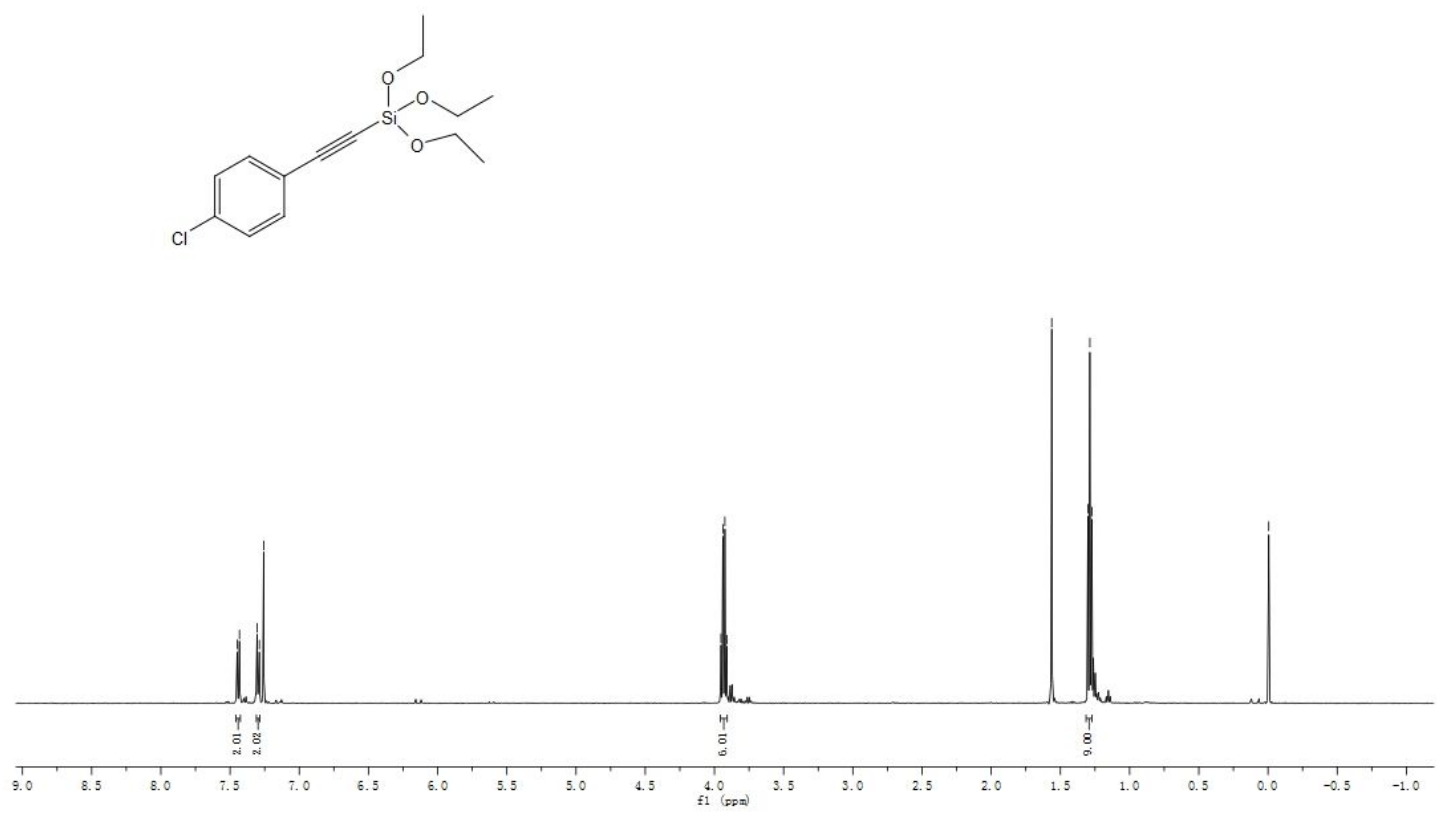

${ }^{1} \mathrm{H}$ NMR $\left(500 \mathrm{MHz}, \mathrm{CDCl}_{3}\right)$ spectrum of $\mathbf{4} \mathbf{j a}$
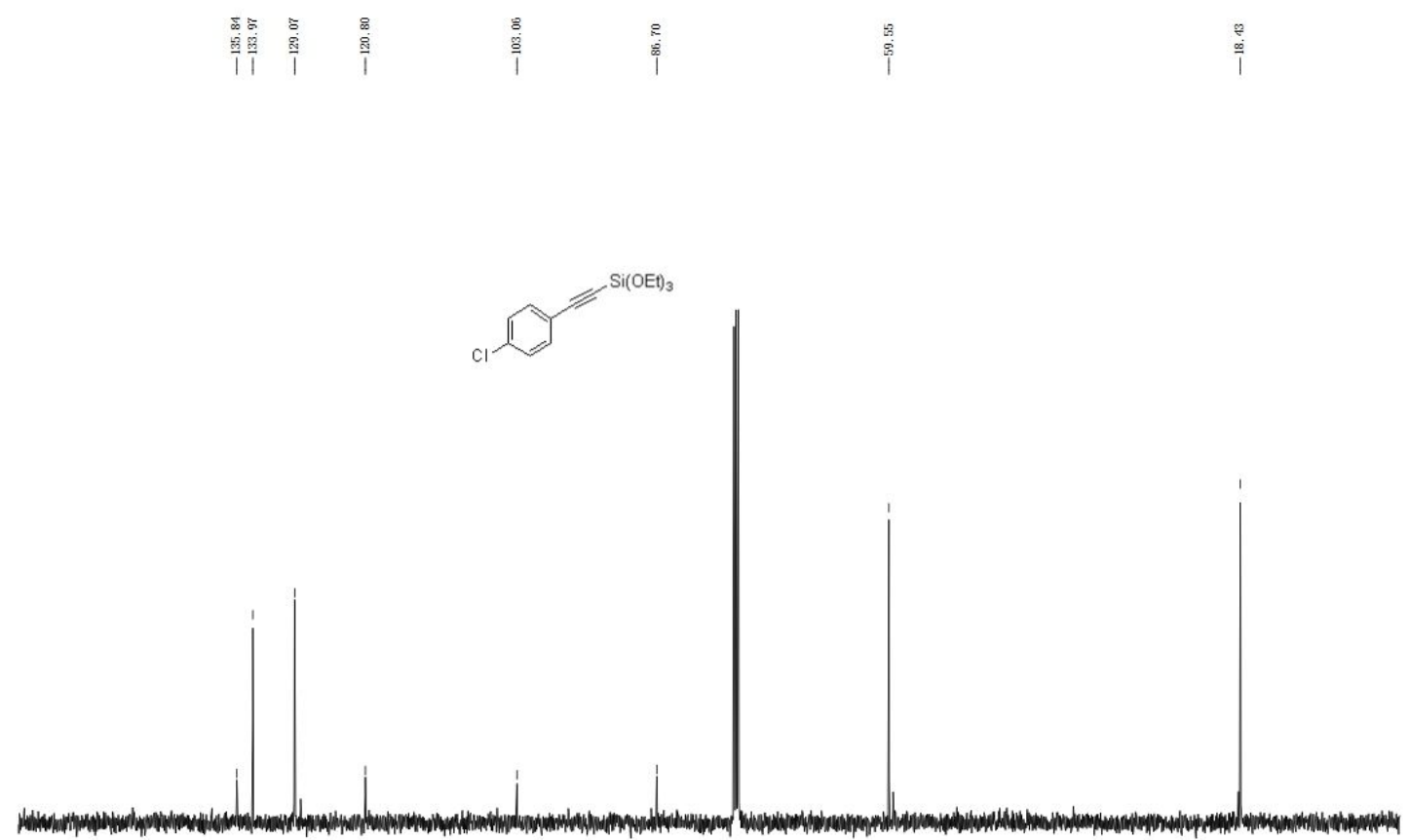

$\underset{160}{1}$

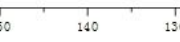

$130+120$

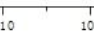

${ }^{13} \mathrm{C}$ NMR $\left(125 \mathrm{MHz}, \mathrm{CDCl}_{3}\right)$ spectrum of $\mathbf{4 j a}$ 

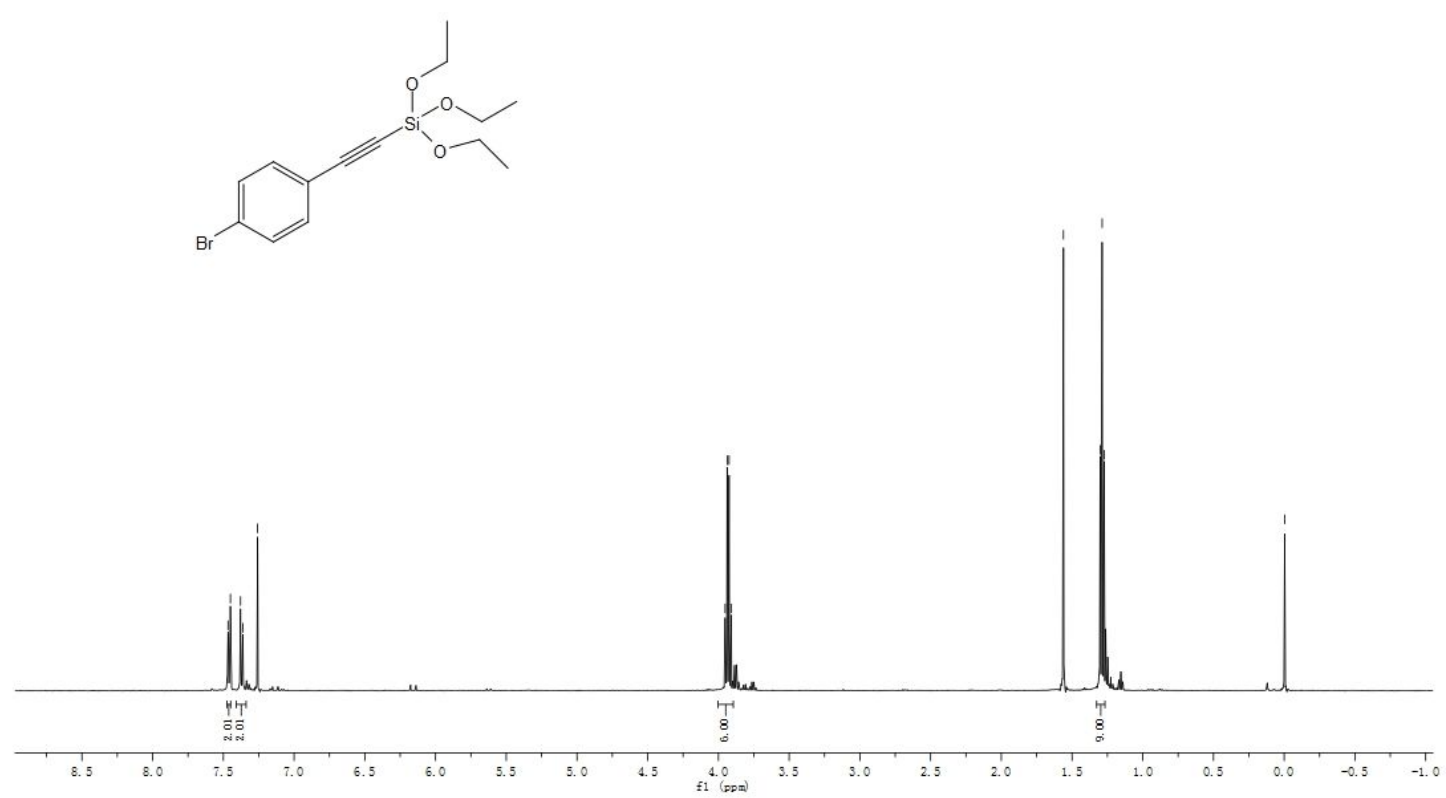

${ }^{1} \mathrm{H}$ NMR $\left(500 \mathrm{MHz}, \mathrm{CDCl}_{3}\right)$ spectrum of $\mathbf{4 k a}$

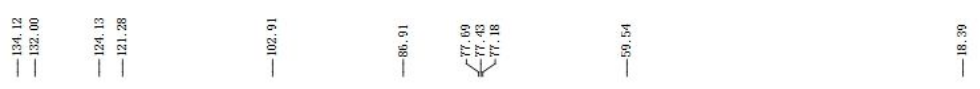
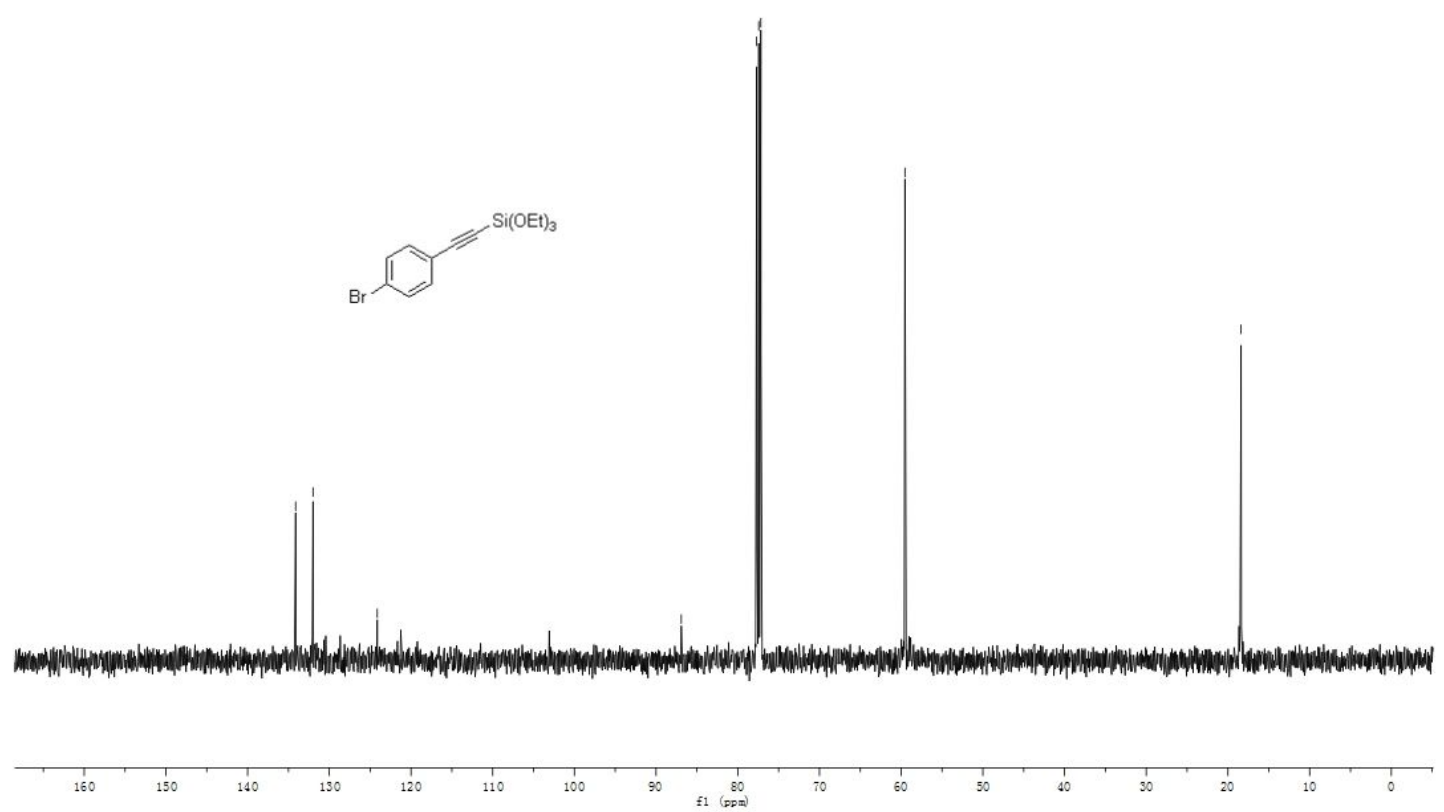

${ }^{13} \mathrm{C}$ NMR $\left(125 \mathrm{MHz}, \mathrm{CDCl}_{3}\right)$ spectrum of $4 \mathbf{k a}$ 


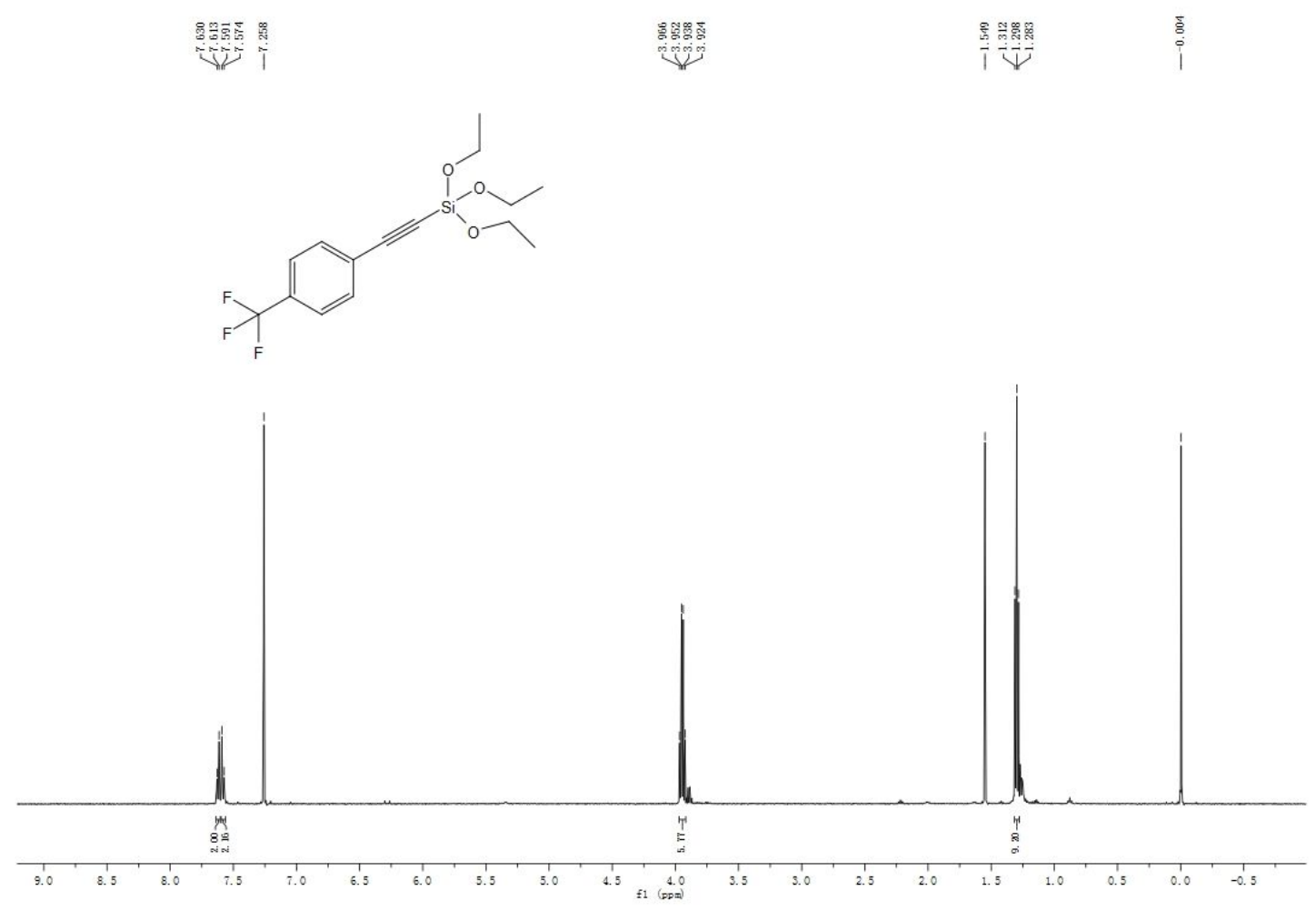

${ }^{1} \mathrm{H}$ NMR $\left(500 \mathrm{MHz}, \mathrm{CDCl}_{3}\right)$ spectrum of 4 la
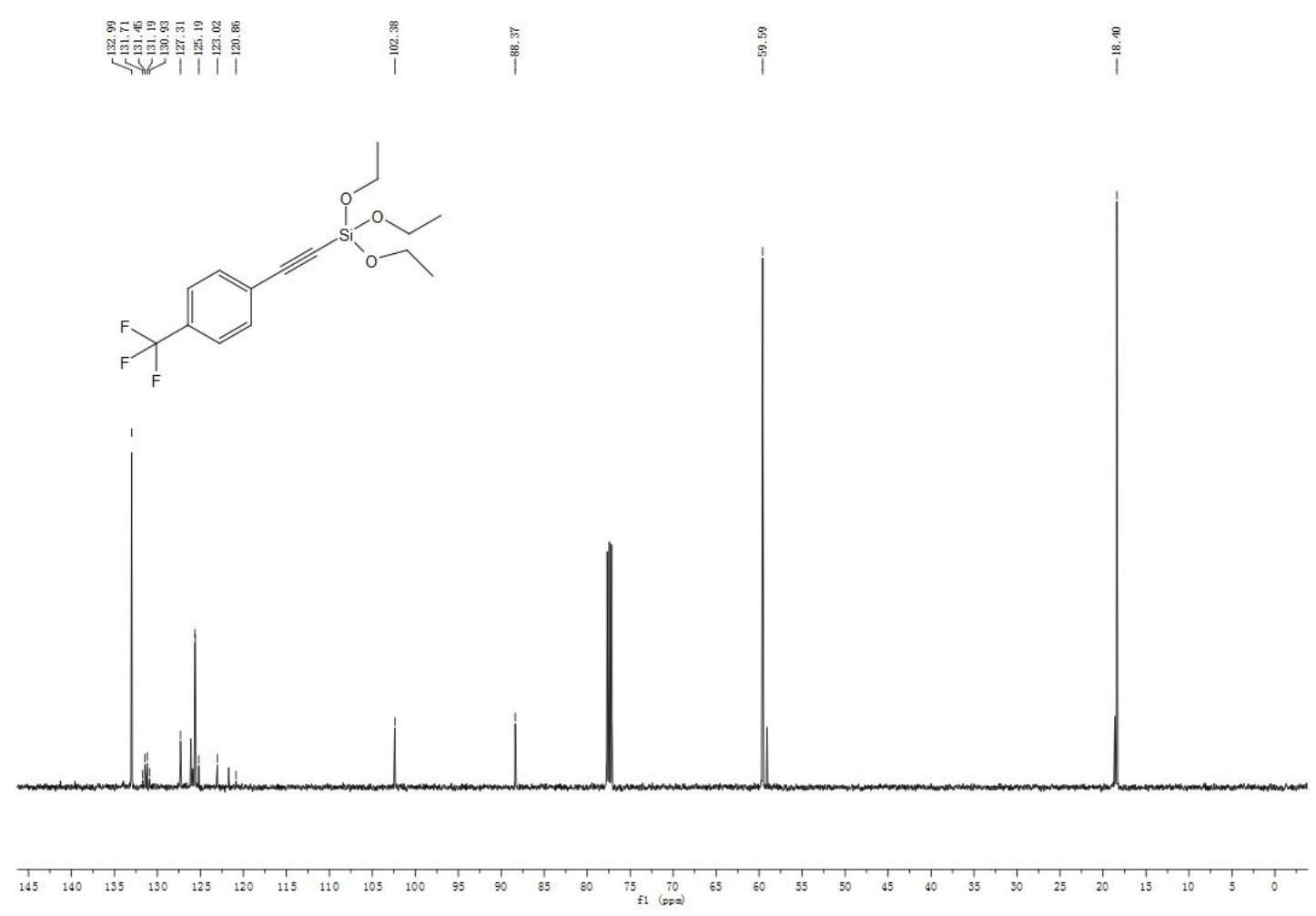

${ }^{13} \mathrm{C}$ NMR $\left(125 \mathrm{MHz}, \mathrm{CDCl}_{3}\right)$ spectrum of 4 la 


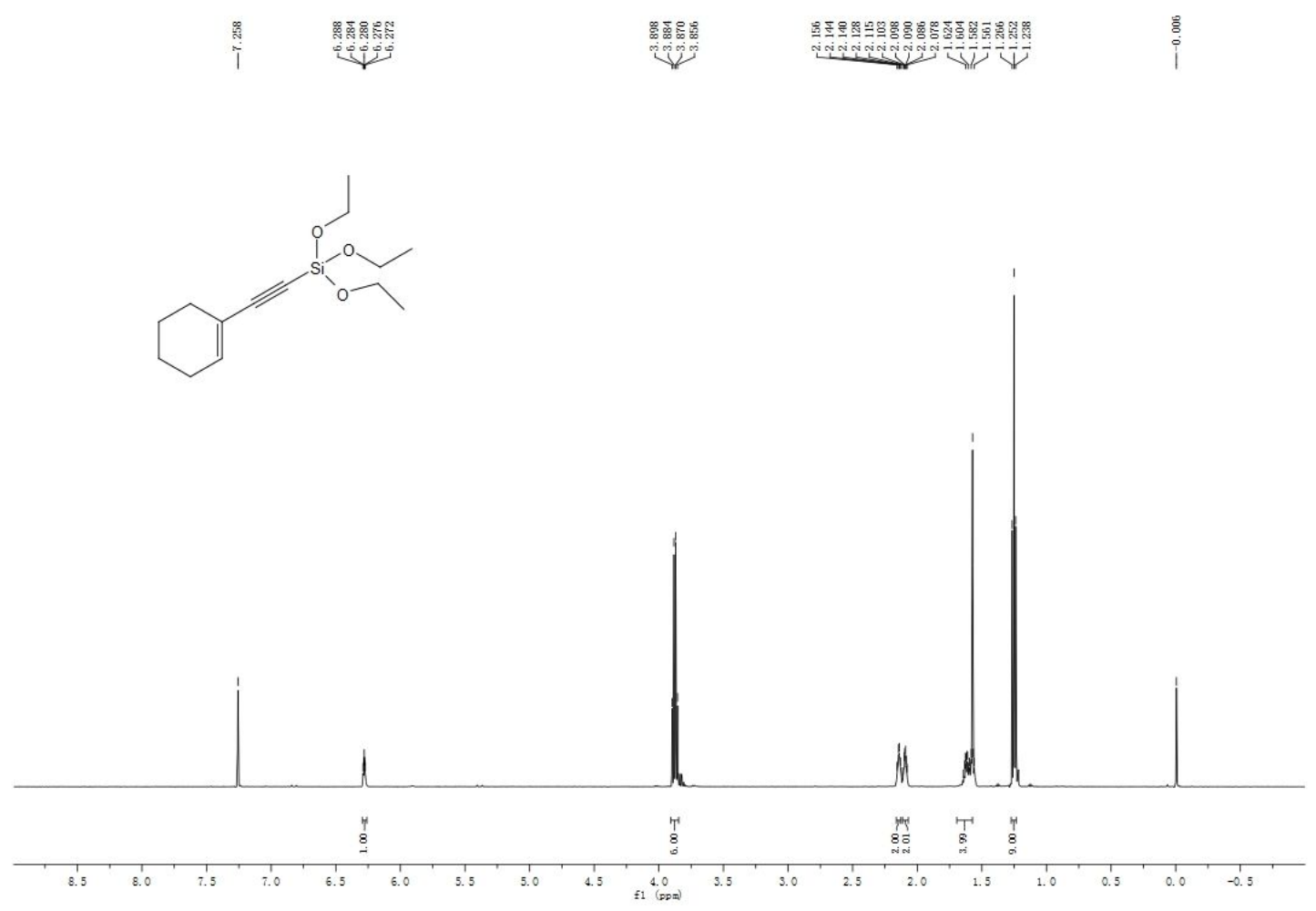

${ }^{1} \mathrm{H}$ NMR $\left(500 \mathrm{MHz}, \mathrm{CDCl}_{3}\right)$ spectrum of $\mathbf{4} \mathbf{m a}$

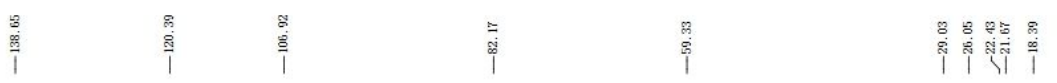
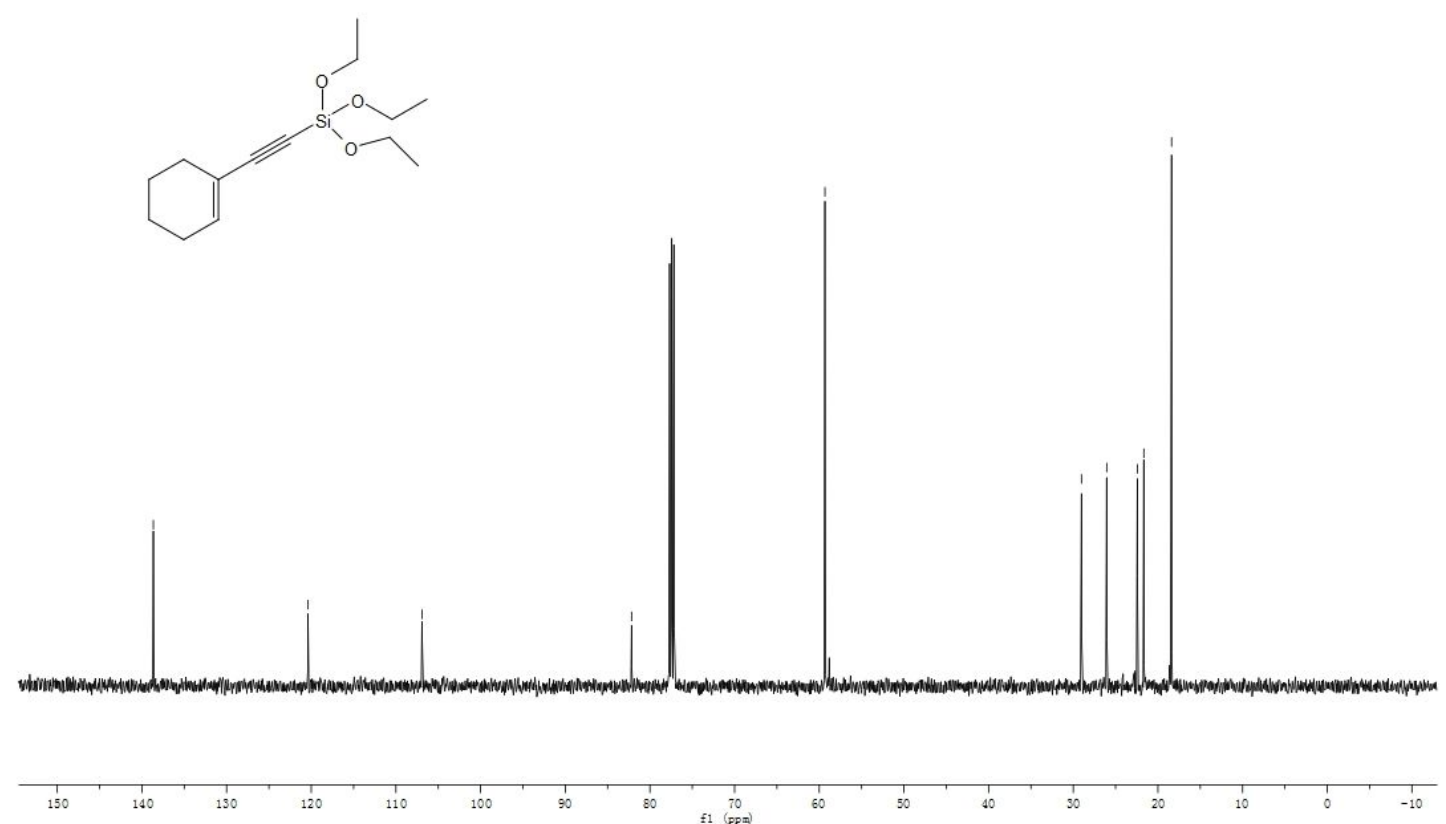

${ }^{13} \mathrm{C} \mathrm{NMR}\left(125 \mathrm{MHz}, \mathrm{CDCl}_{3}\right)$ spectrum of $4 \mathbf{m a}$ 


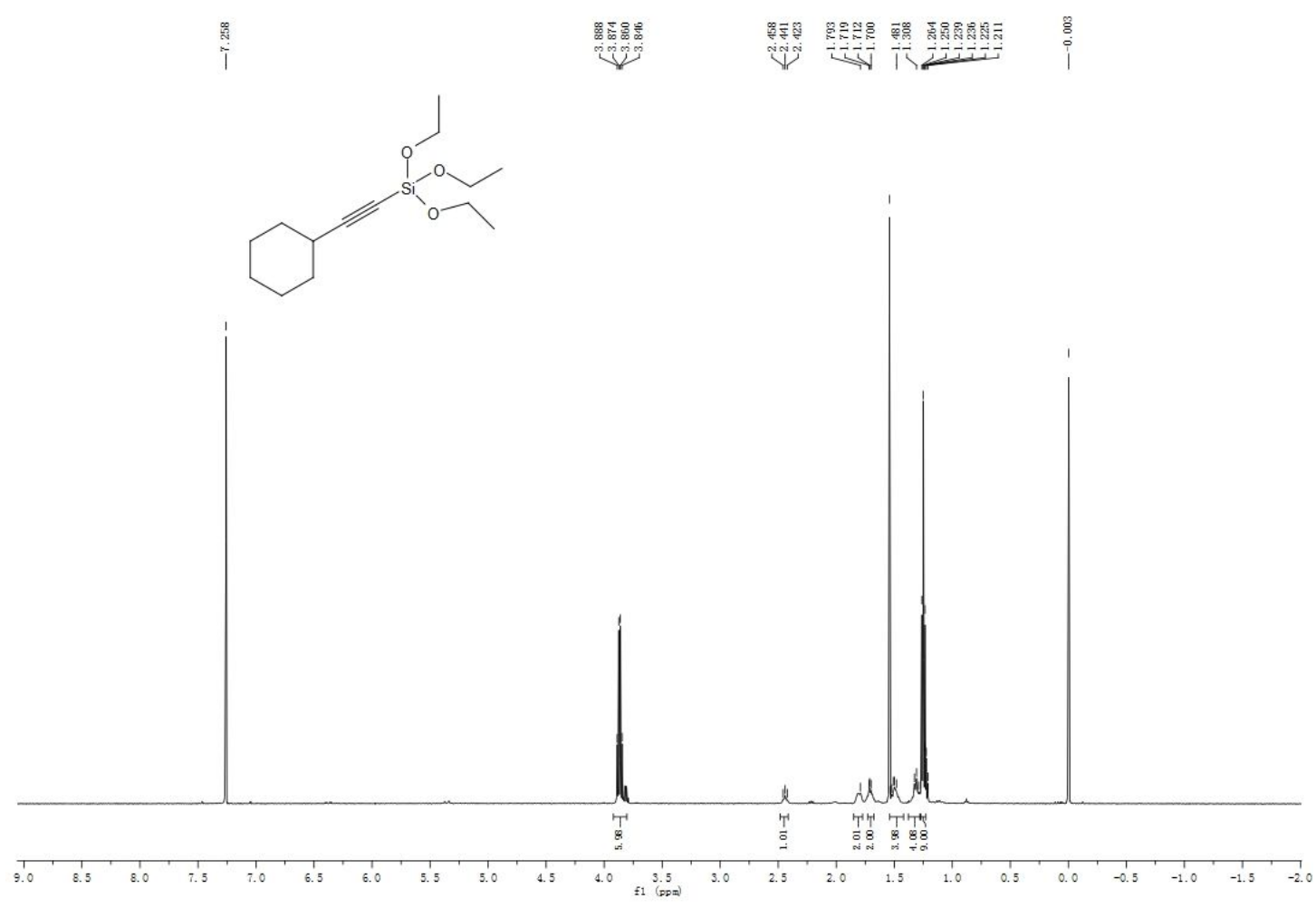

${ }^{1} \mathrm{H}$ NMR $\left(500 \mathrm{MHz}, \mathrm{CDCl}_{3}\right)$ spectrum of $4 \mathbf{n a}$
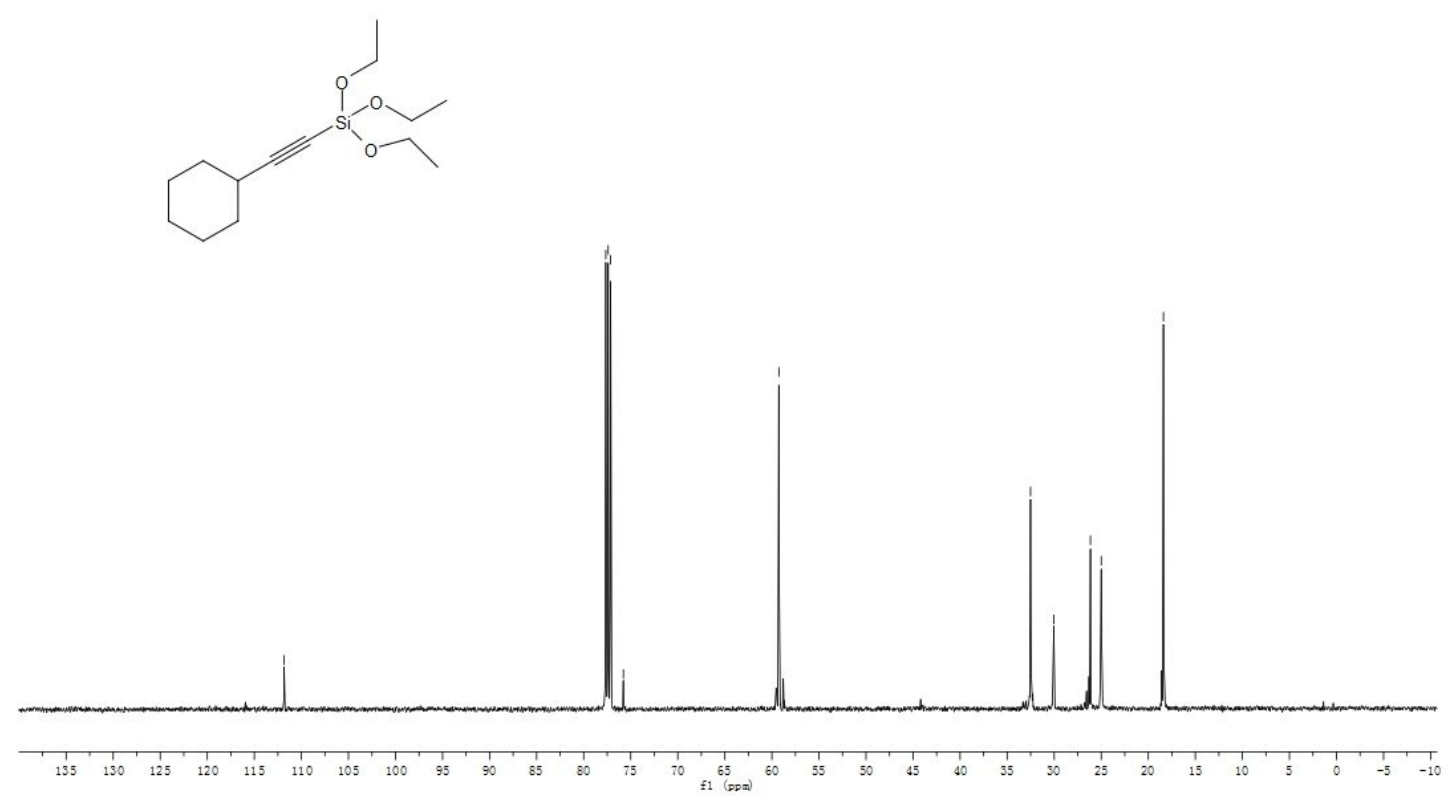

${ }^{13} \mathrm{C}$ NMR $\left(125 \mathrm{MHz}, \mathrm{CDCl}_{3}\right)$ spectrum of $4 \mathbf{n a}$ 


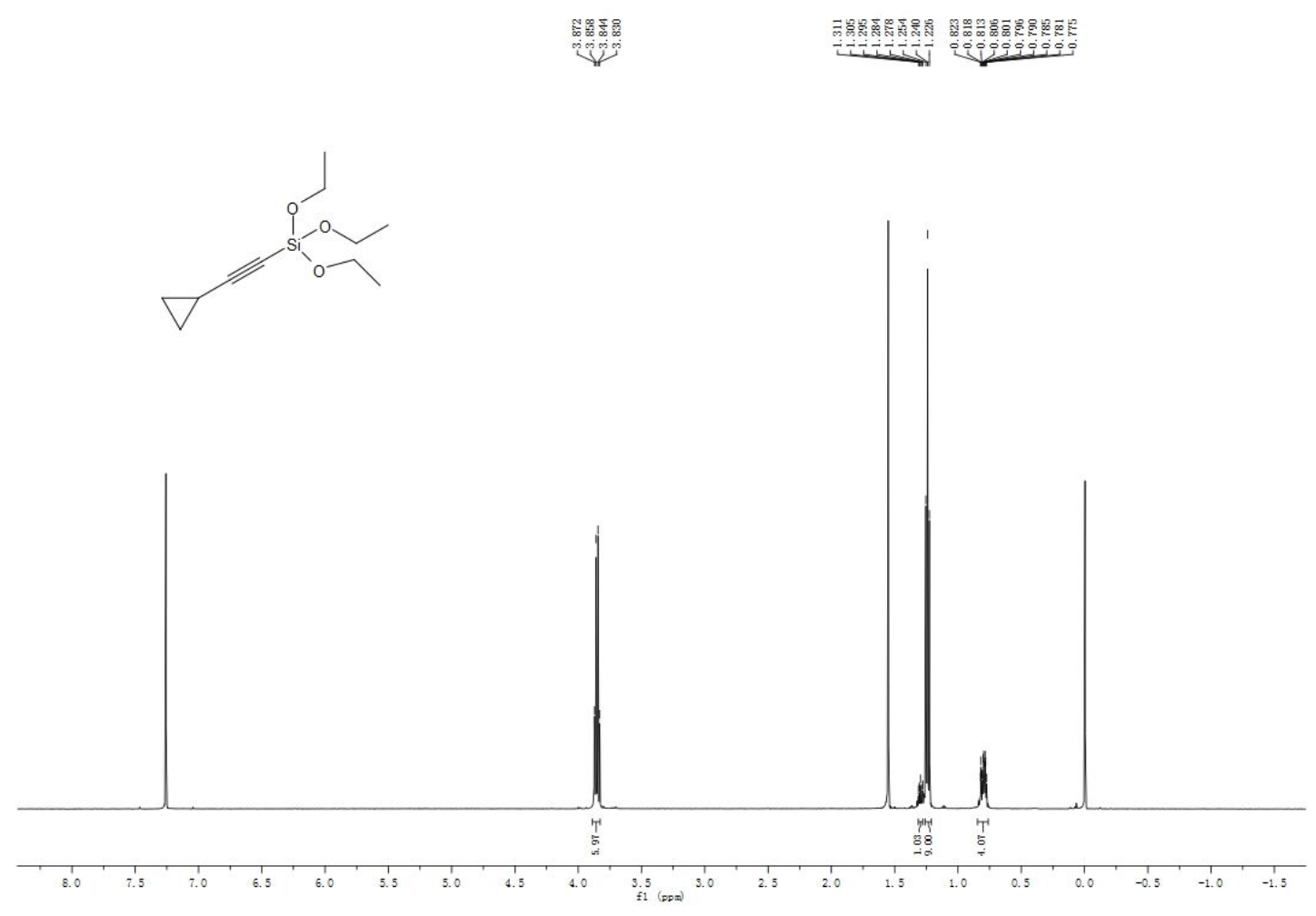

${ }^{1} \mathrm{H} \mathrm{NMR}\left(500 \mathrm{MHz}, \mathrm{CDCl}_{3}\right)$ spectrum of $4 \mathbf{o a}$

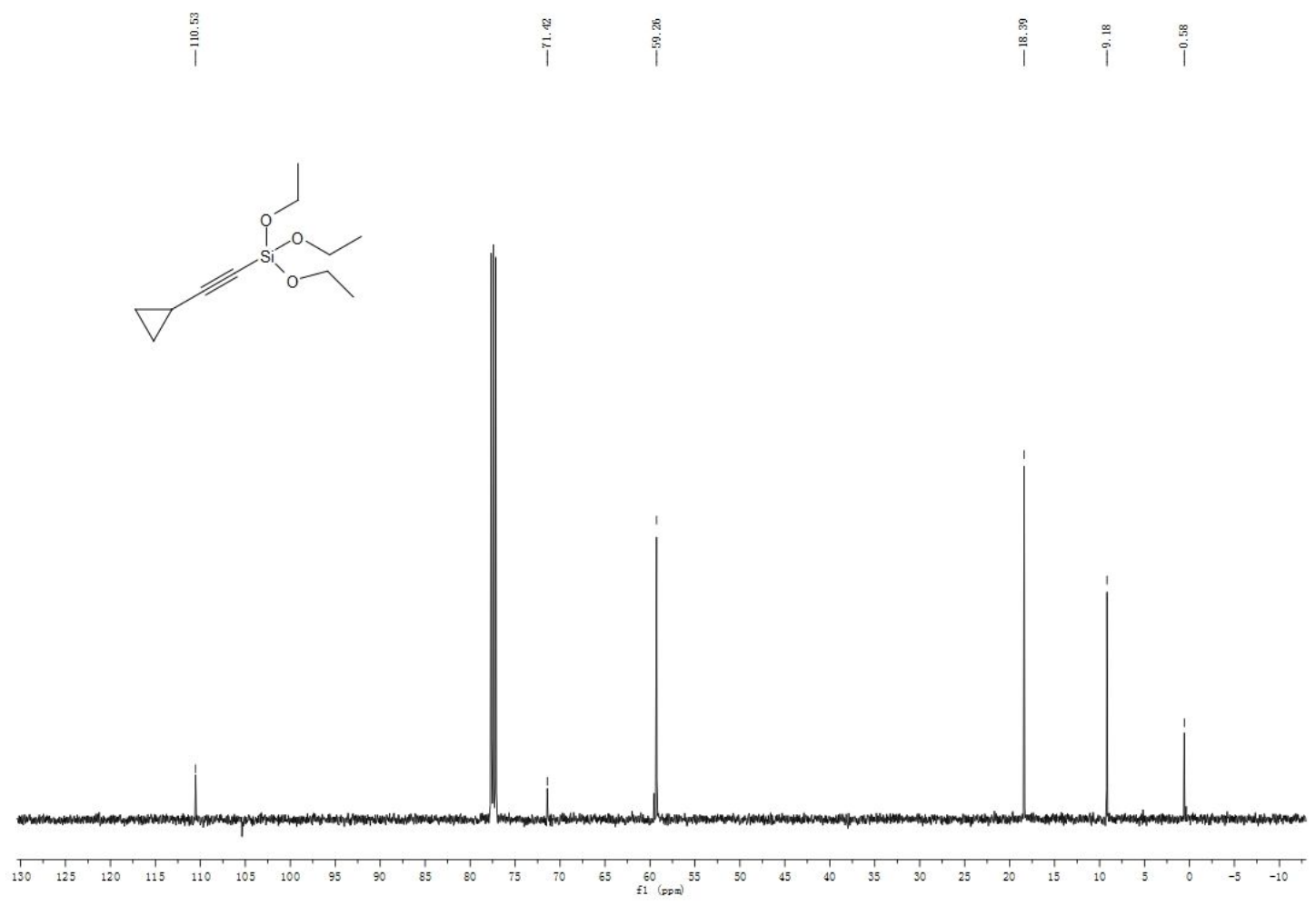

${ }^{13} \mathrm{C}$ NMR $\left(125 \mathrm{MHz}, \mathrm{CDCl}_{3}\right)$ spectrum of $4 \mathbf{o a}$ 


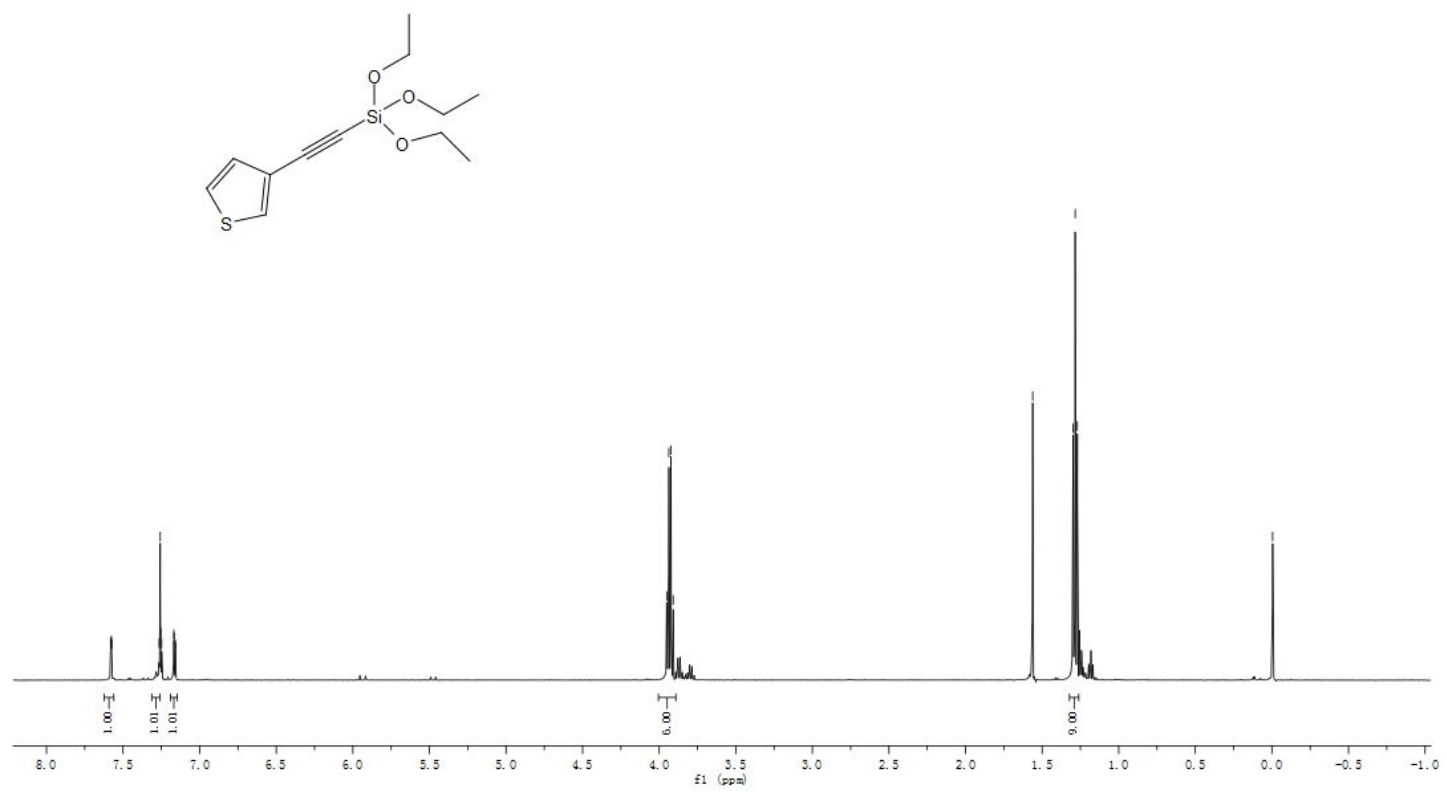

${ }^{1} \mathrm{H}$ NMR $\left(500 \mathrm{MHz}, \mathrm{CDCl}_{3}\right)$ spectrum of 4 pa

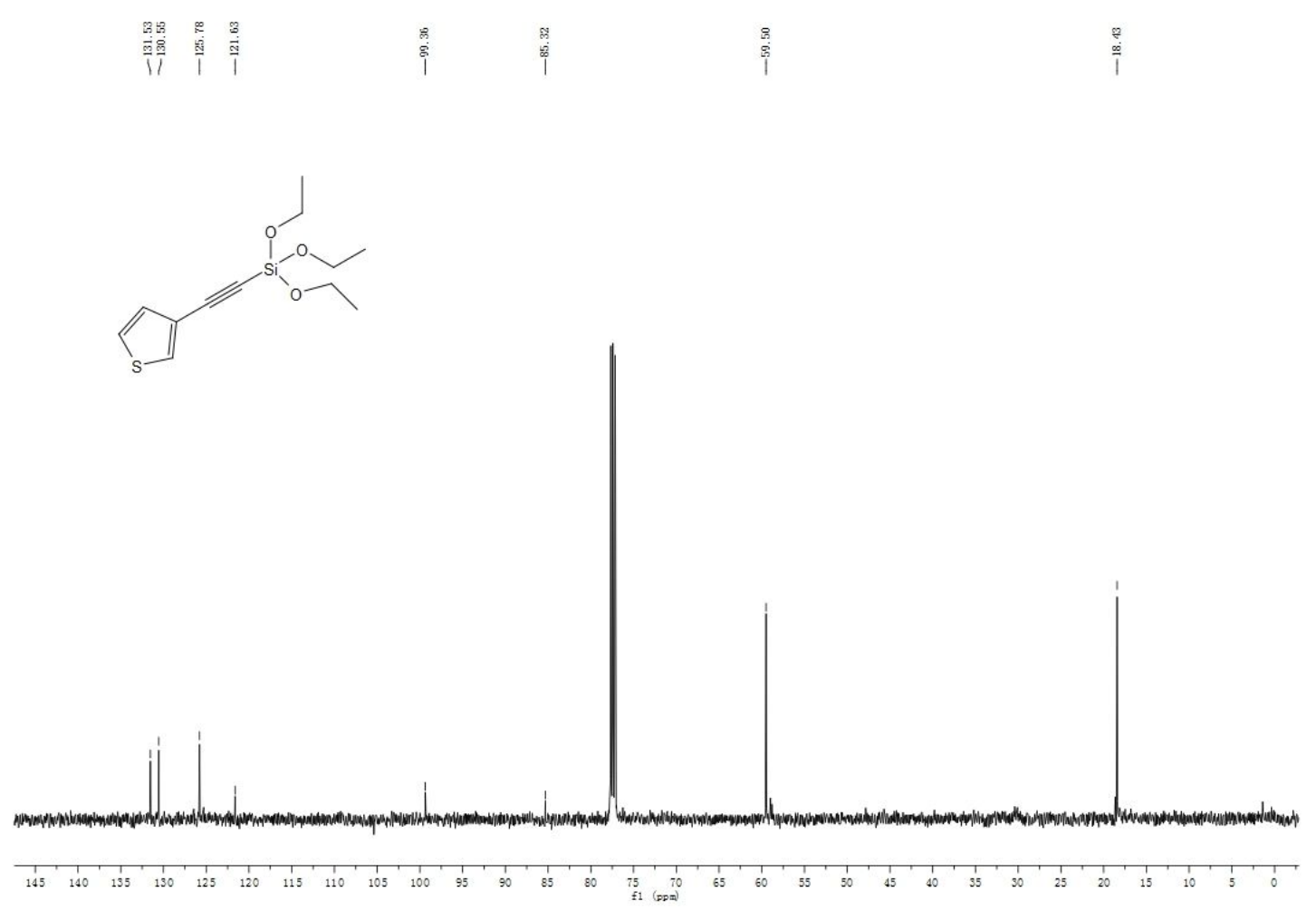

${ }^{13} \mathrm{C}$ NMR $\left(125 \mathrm{MHz}, \mathrm{CDCl}_{3}\right)$ spectrum of 4 pa 

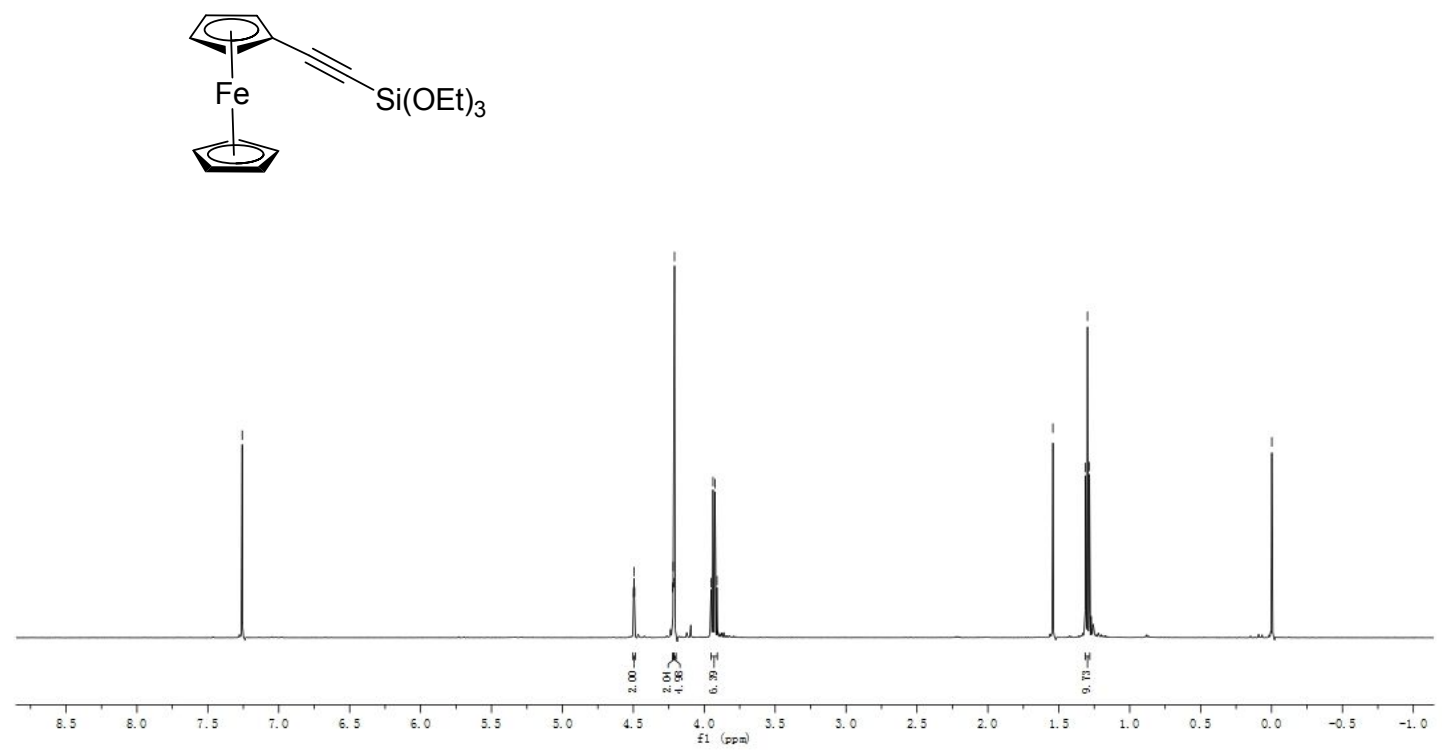

${ }^{1} \mathrm{H}$ NMR $\left(500 \mathrm{MHz}, \mathrm{CDCl}_{3}\right)$ spectrum of $\mathbf{4 q a}$

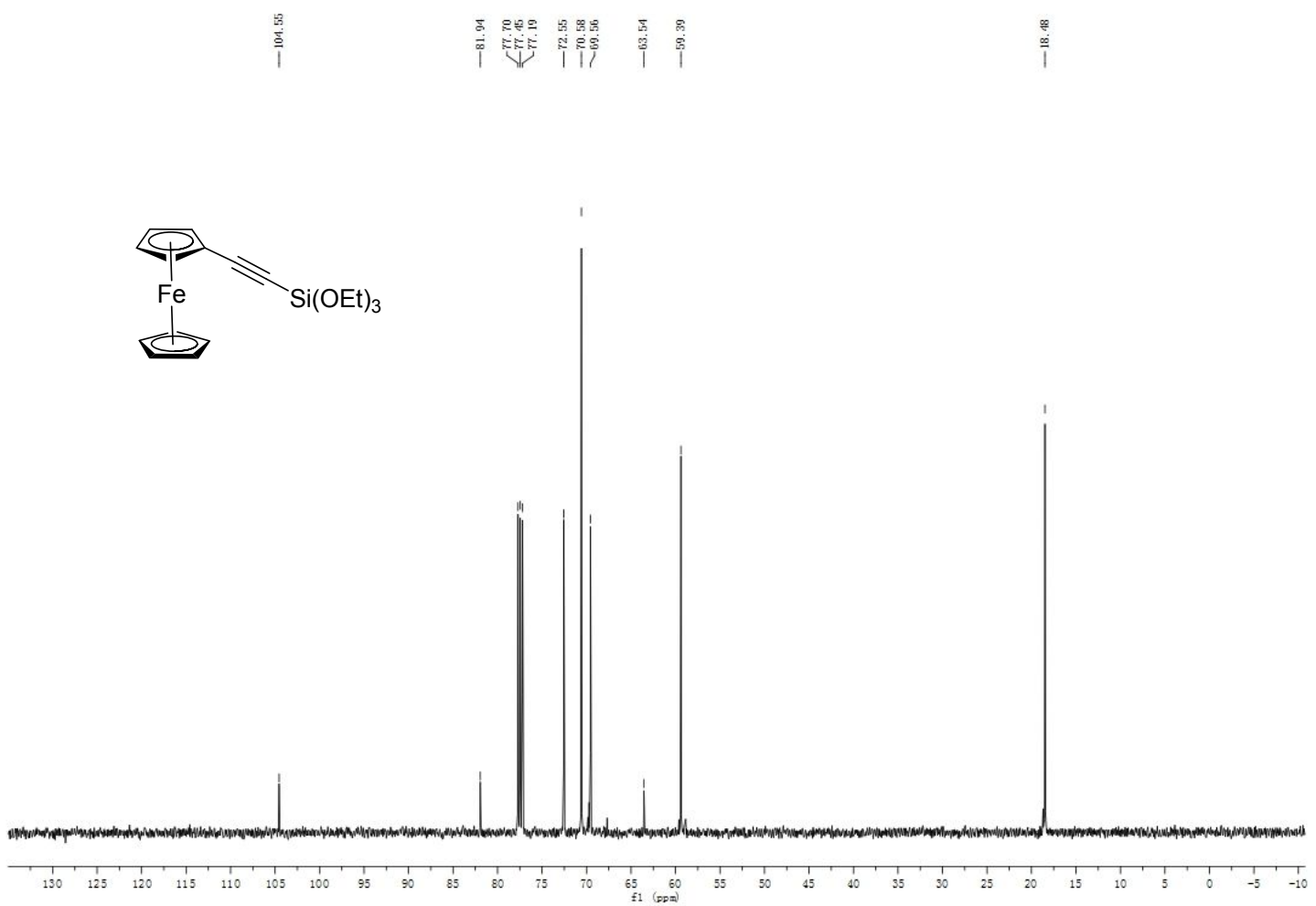

${ }^{13} \mathrm{C}$ NMR $\left(125 \mathrm{MHz}, \mathrm{CDCl}_{3}\right)$ spectrum of $\mathbf{4 q a}$ 


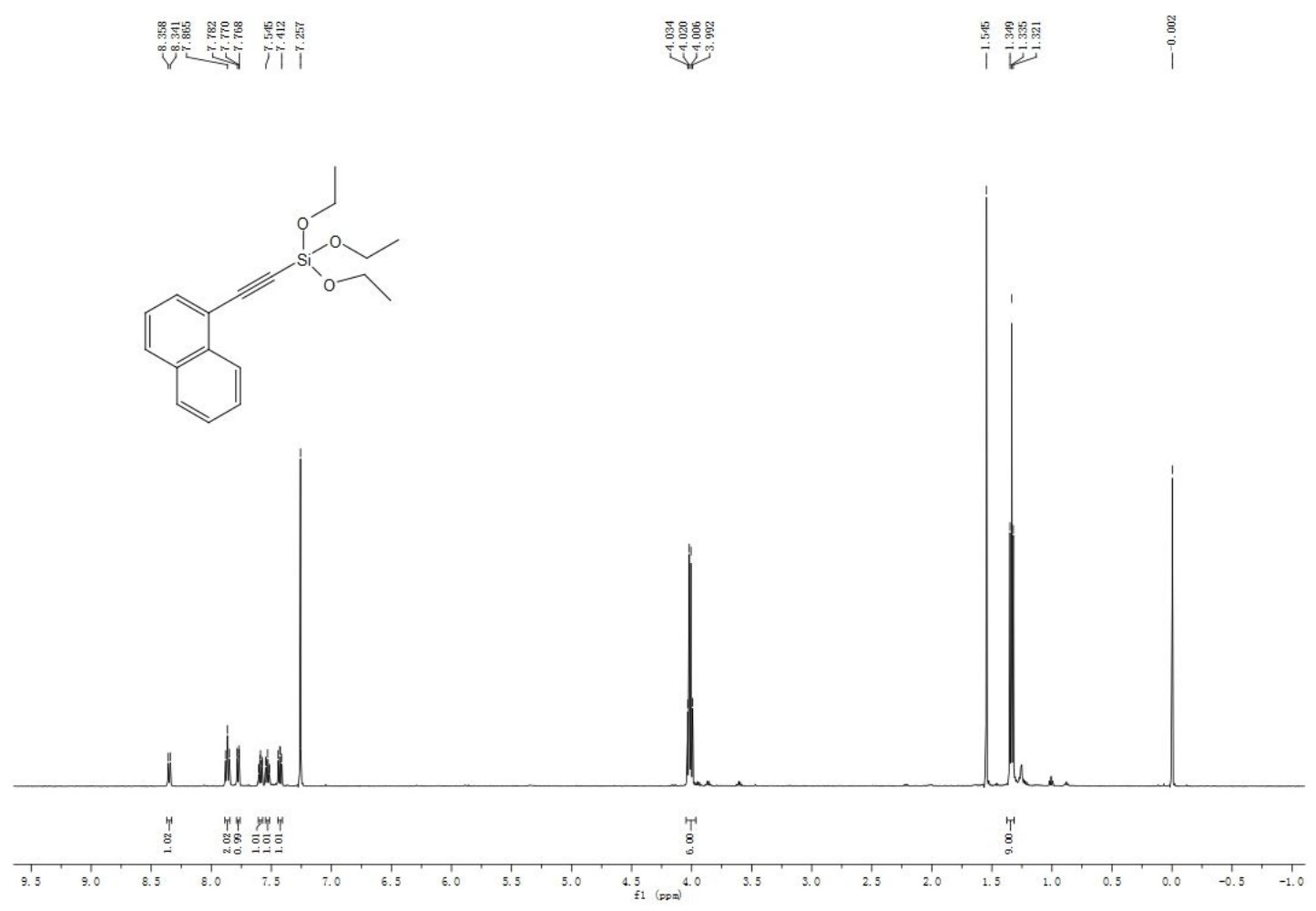

${ }^{1} \mathrm{H} \mathrm{NMR}\left(500 \mathrm{MHz}, \mathrm{CDCl}_{3}\right)$ spectrum of 4ra

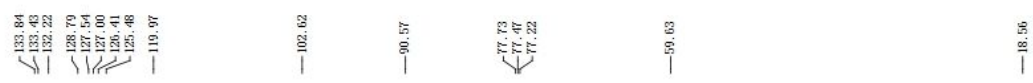
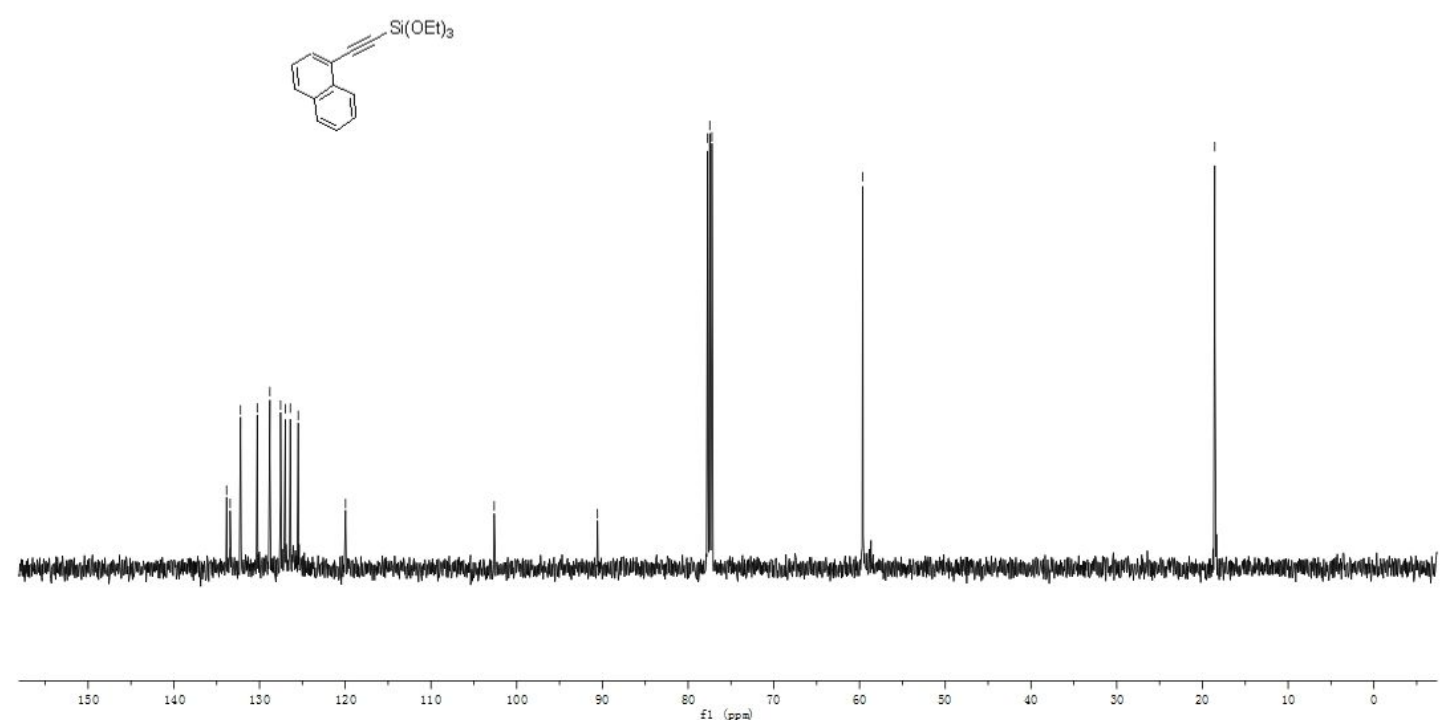

${ }^{13} \mathrm{C}$ NMR $\left(125 \mathrm{MHz}, \mathrm{CDCl}_{3}\right)$ spectrum of 4 ra 


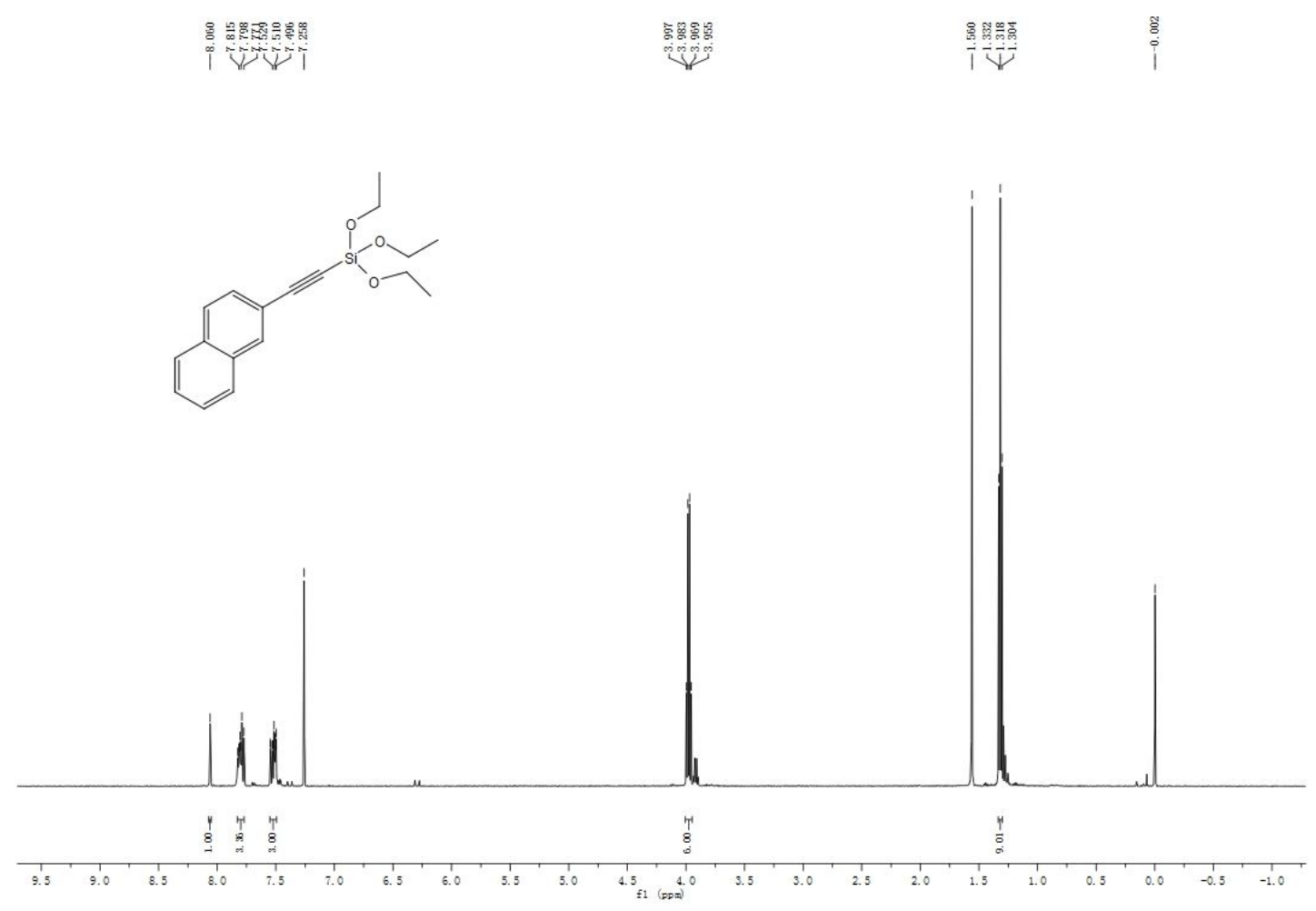

${ }^{1} \mathrm{H}$ NMR $\left(500 \mathrm{MHz}, \mathrm{CDCl}_{3}\right)$ spectrum of $4 \mathbf{s a}$

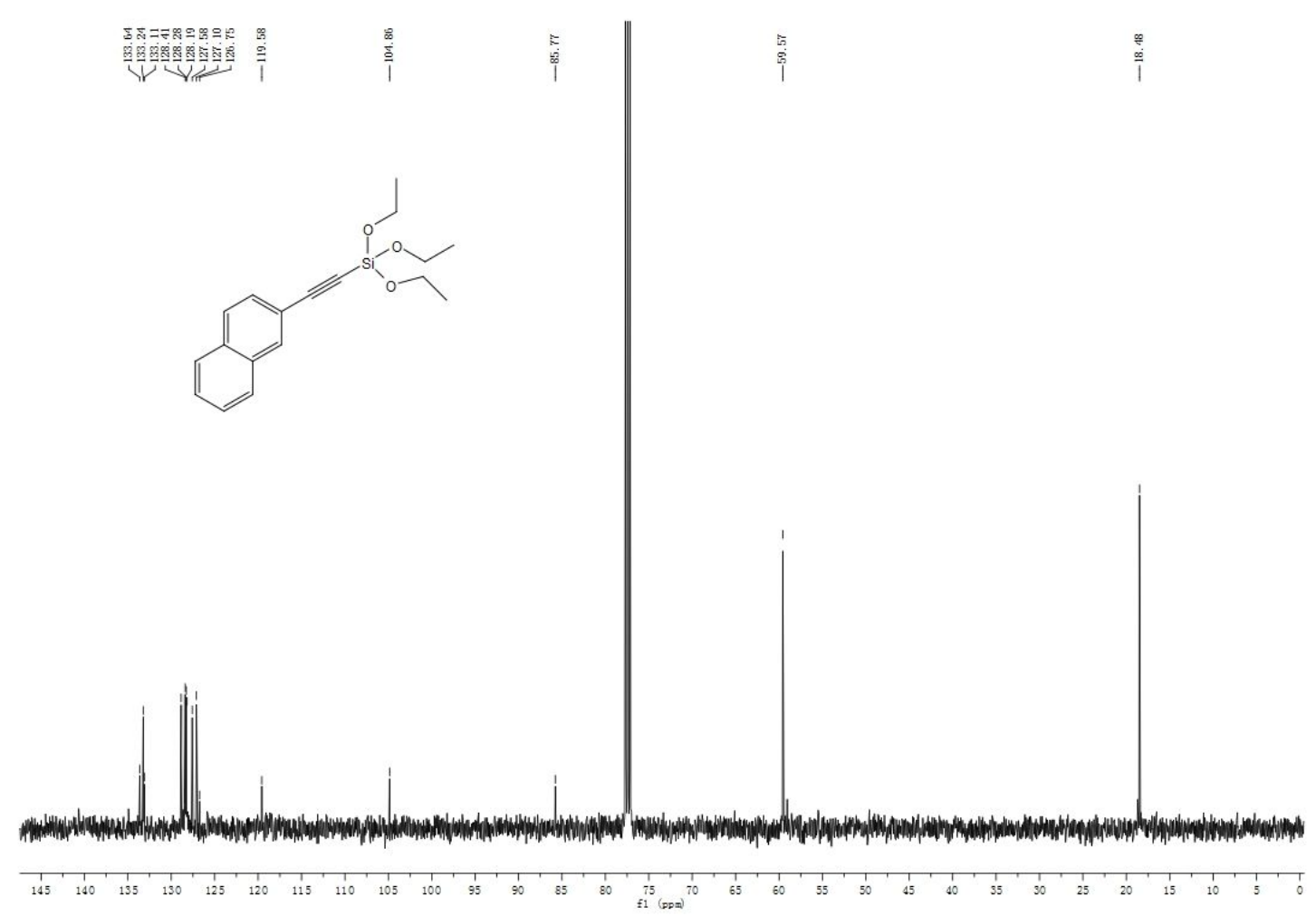

${ }^{13} \mathrm{C}$ NMR $\left(125 \mathrm{MHz}, \mathrm{CDCl}_{3}\right)$ spectrum of $4 \mathbf{s a}$ 


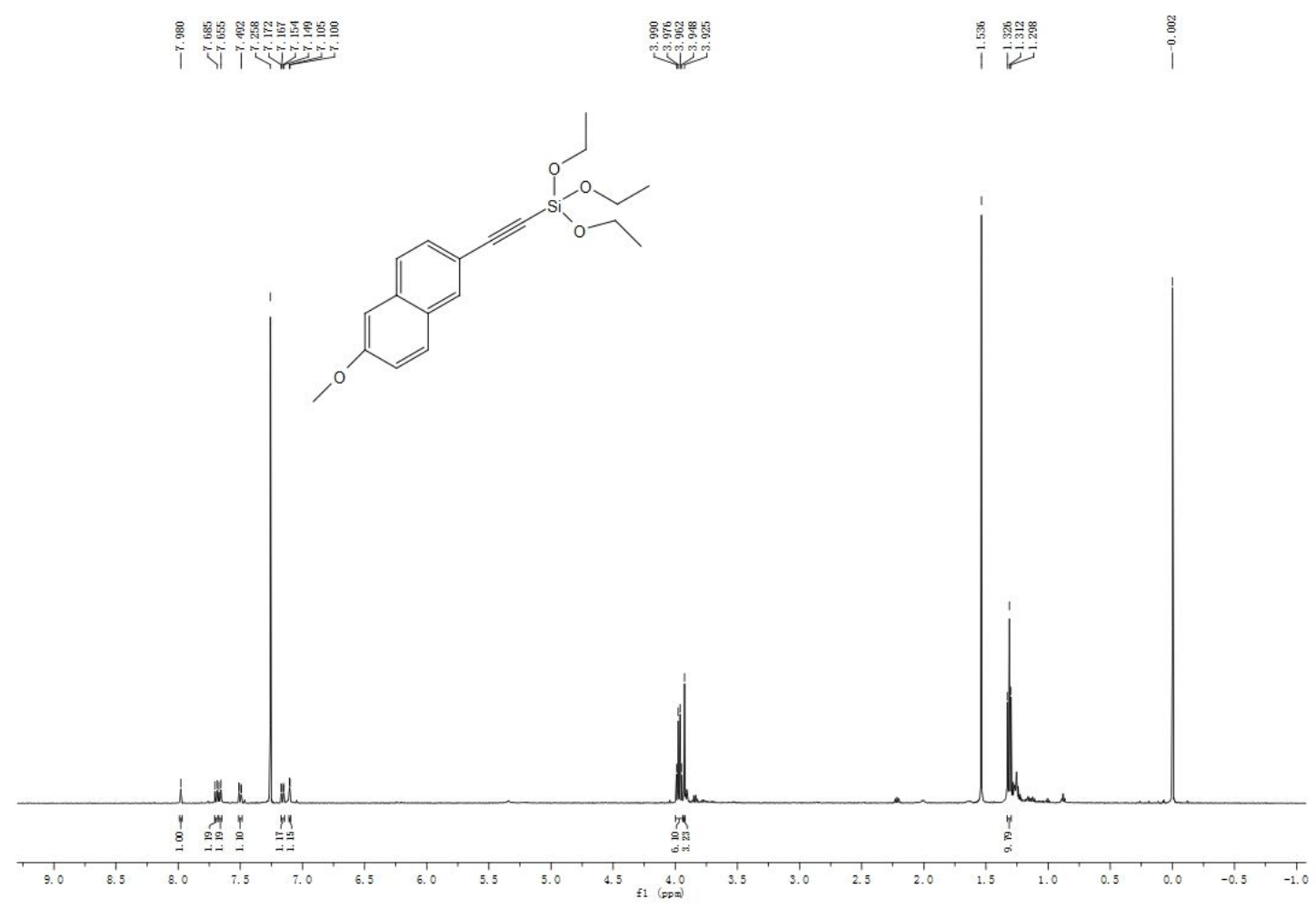

${ }^{1} \mathrm{H} \mathrm{NMR}\left(500 \mathrm{MHz}, \mathrm{CDCl}_{3}\right)$ spectrum of $\mathbf{4 t a}$

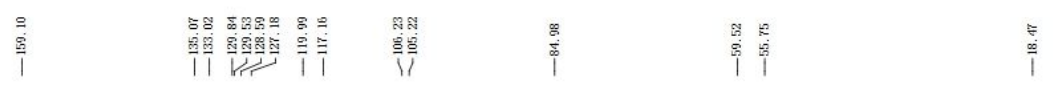

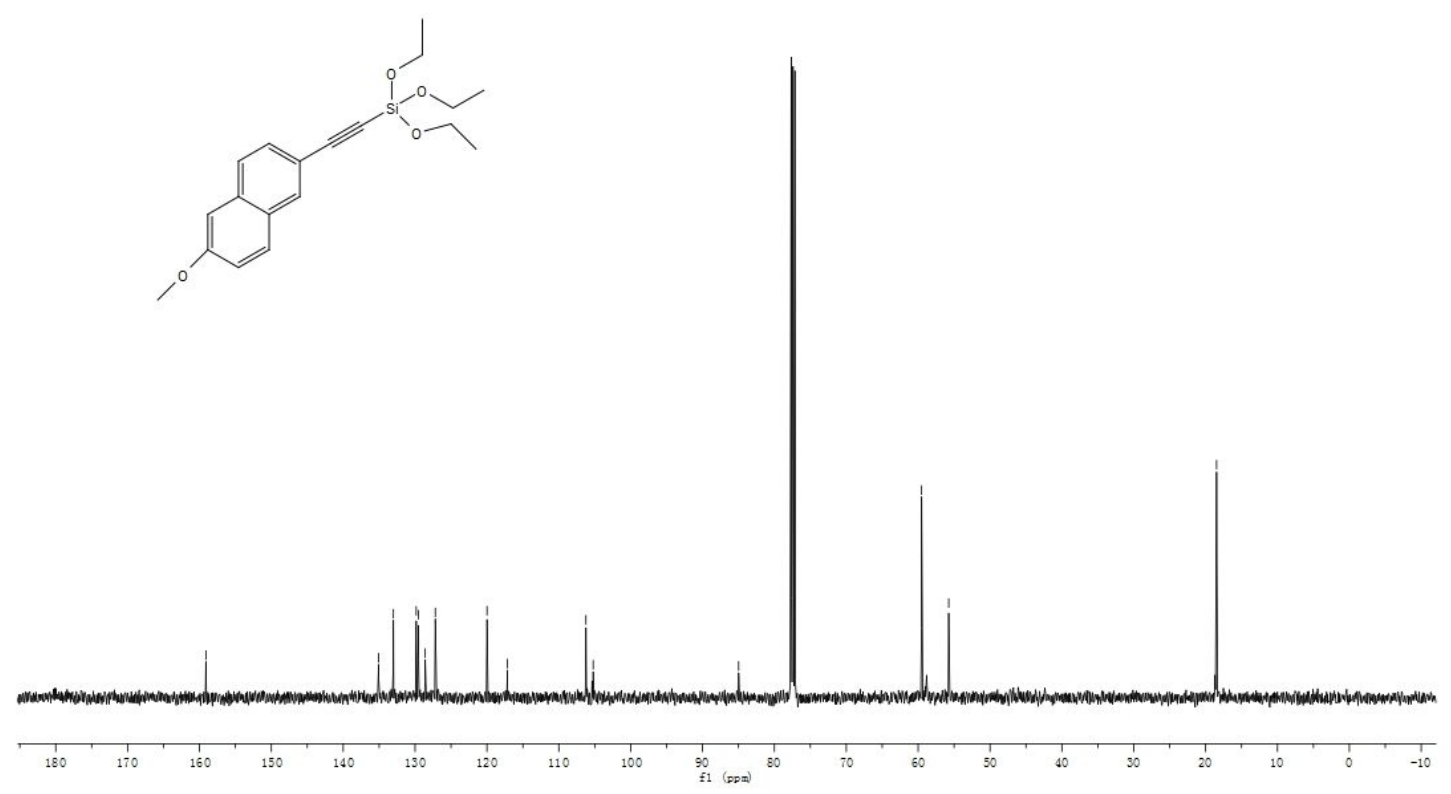

${ }^{13} \mathrm{C}$ NMR $\left(125 \mathrm{MHz}, \mathrm{CDCl}_{3}\right)$ spectrum of 4 ta 


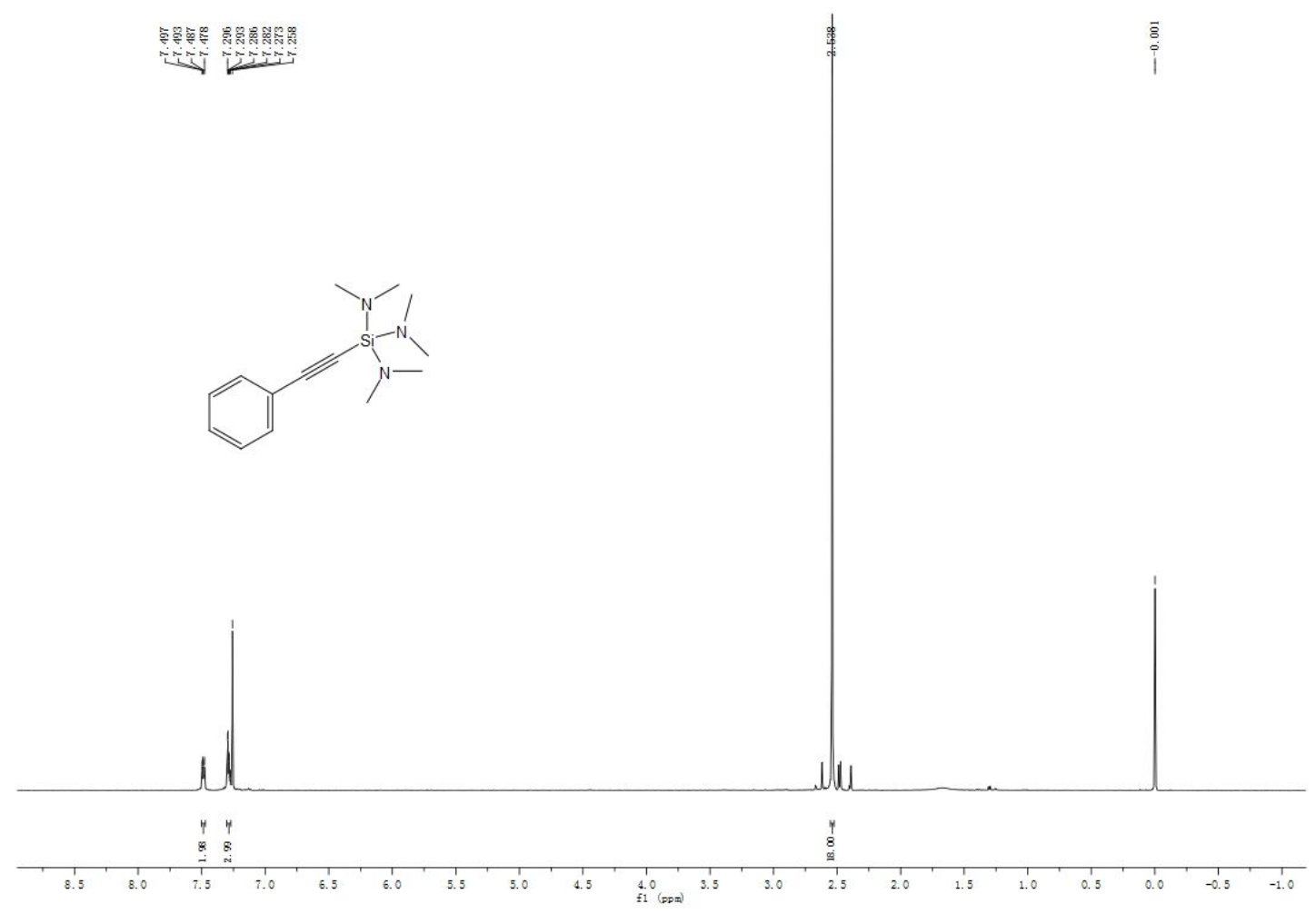

${ }^{1} \mathrm{H}$ NMR $\left(500 \mathrm{MHz}, \mathrm{CDCl}_{3}\right)$ spectrum of $\mathbf{4 a d}$

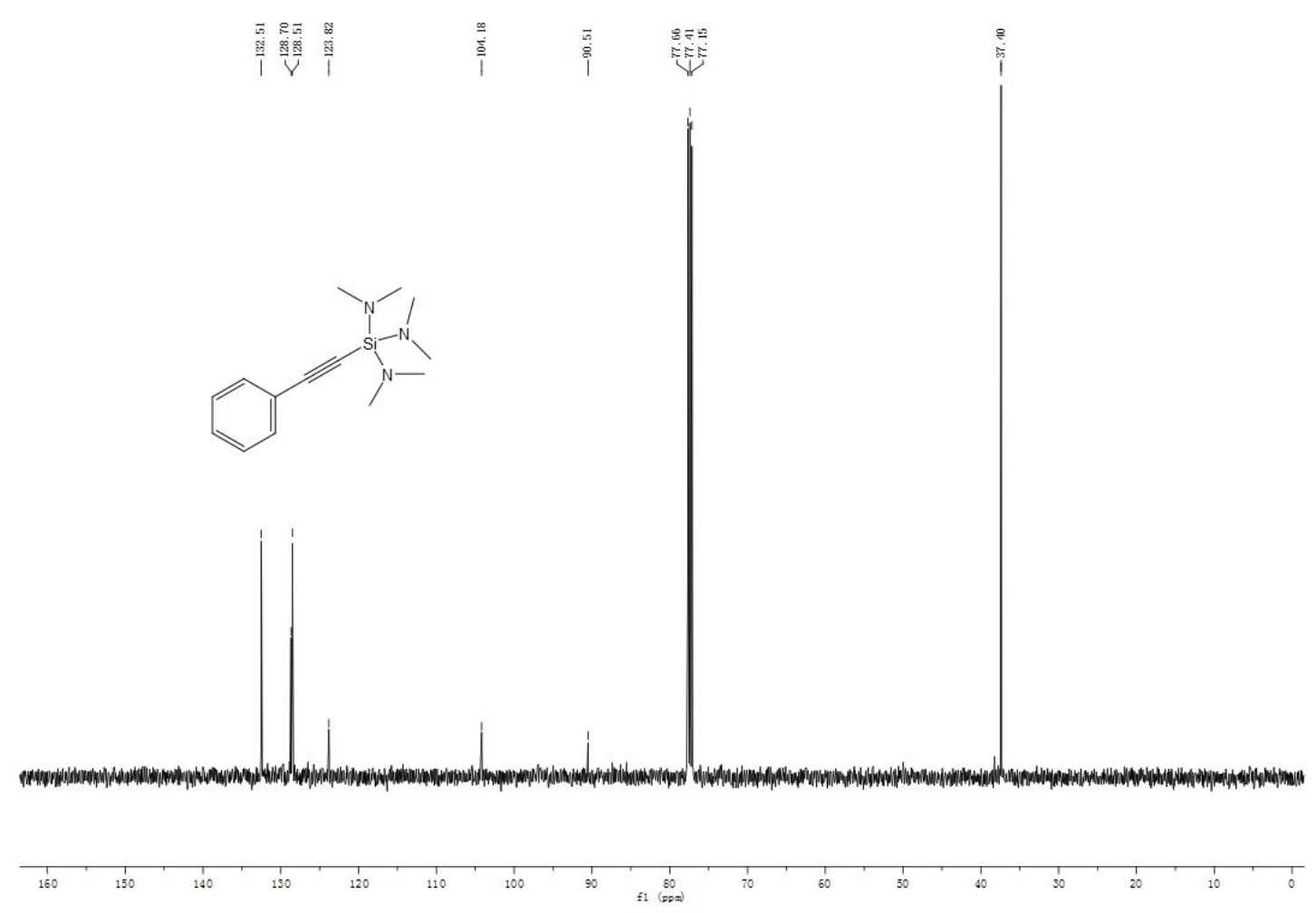

${ }^{13} \mathrm{C}$ NMR $\left(125 \mathrm{MHz}, \mathrm{CDCl}_{3}\right)$ spectrum of $\mathbf{4 a d}$ 


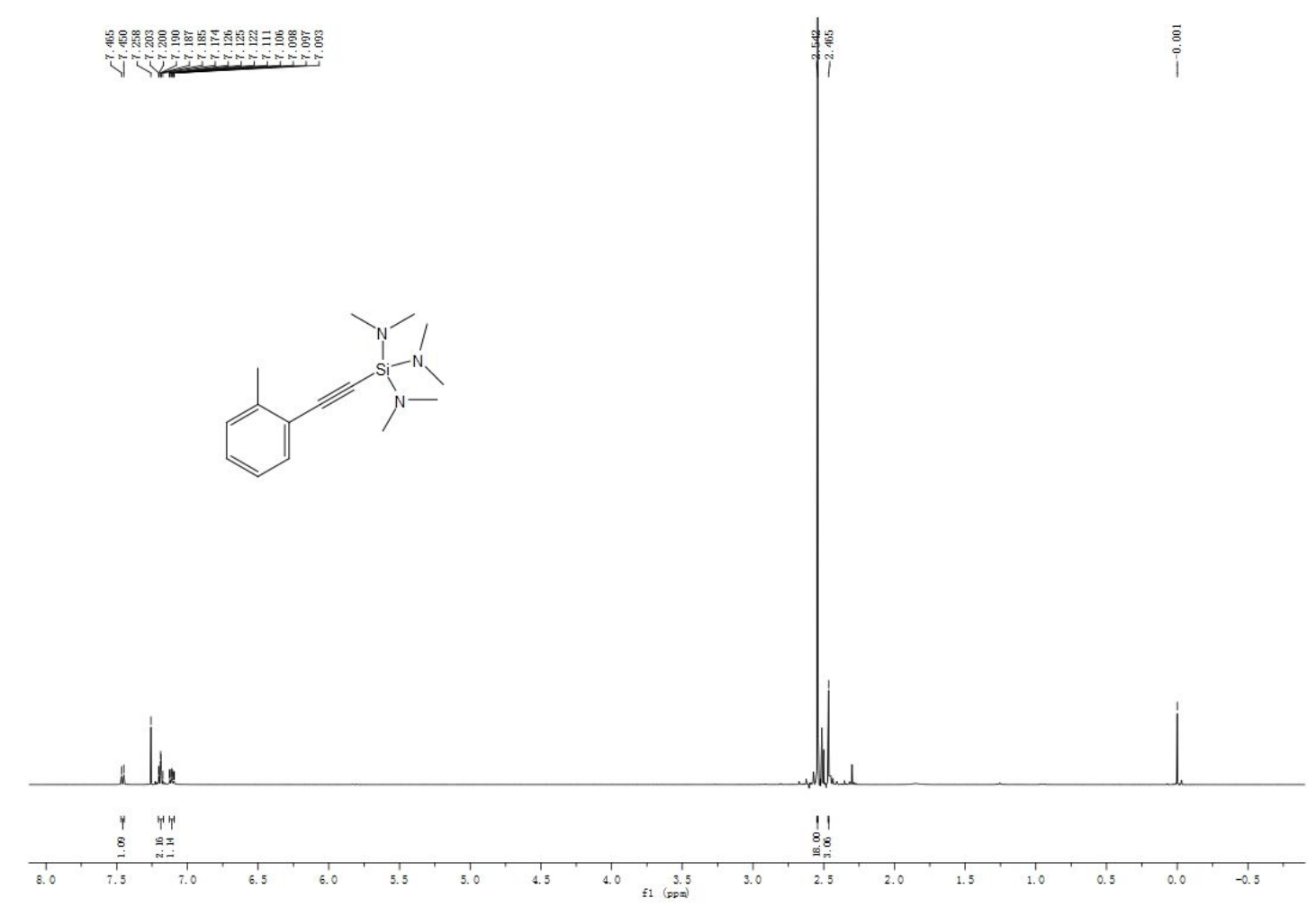

${ }^{1} \mathrm{H}$ NMR $\left(500 \mathrm{MHz}, \mathrm{CDCl}_{3}\right)$ spectrum of $\mathbf{4 b d}$ $\begin{array}{llll}1 & 1 & 1 & 1 \\ 5 & 0\end{array}$
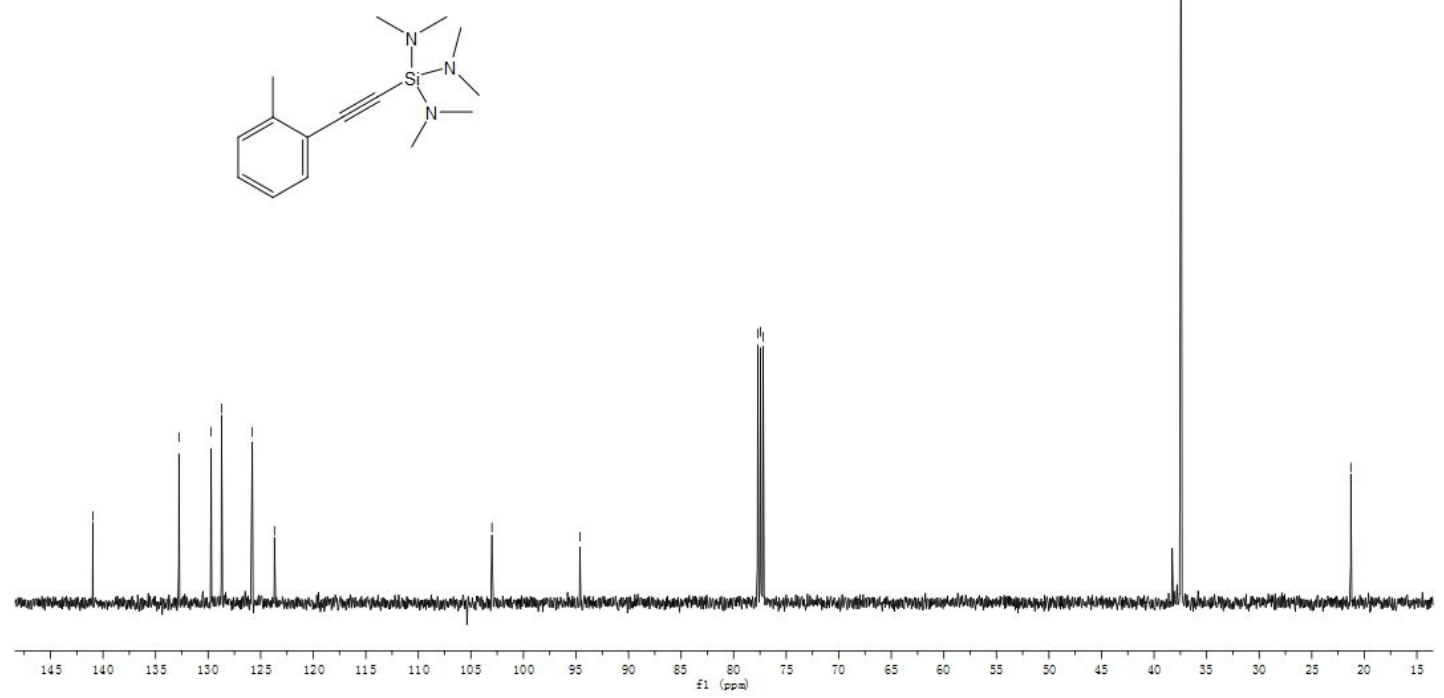

${ }^{13} \mathrm{C}$ NMR $\left(125 \mathrm{MHz}, \mathrm{CDCl}_{3}\right)$ spectrum of $\mathbf{4 b d}$ 


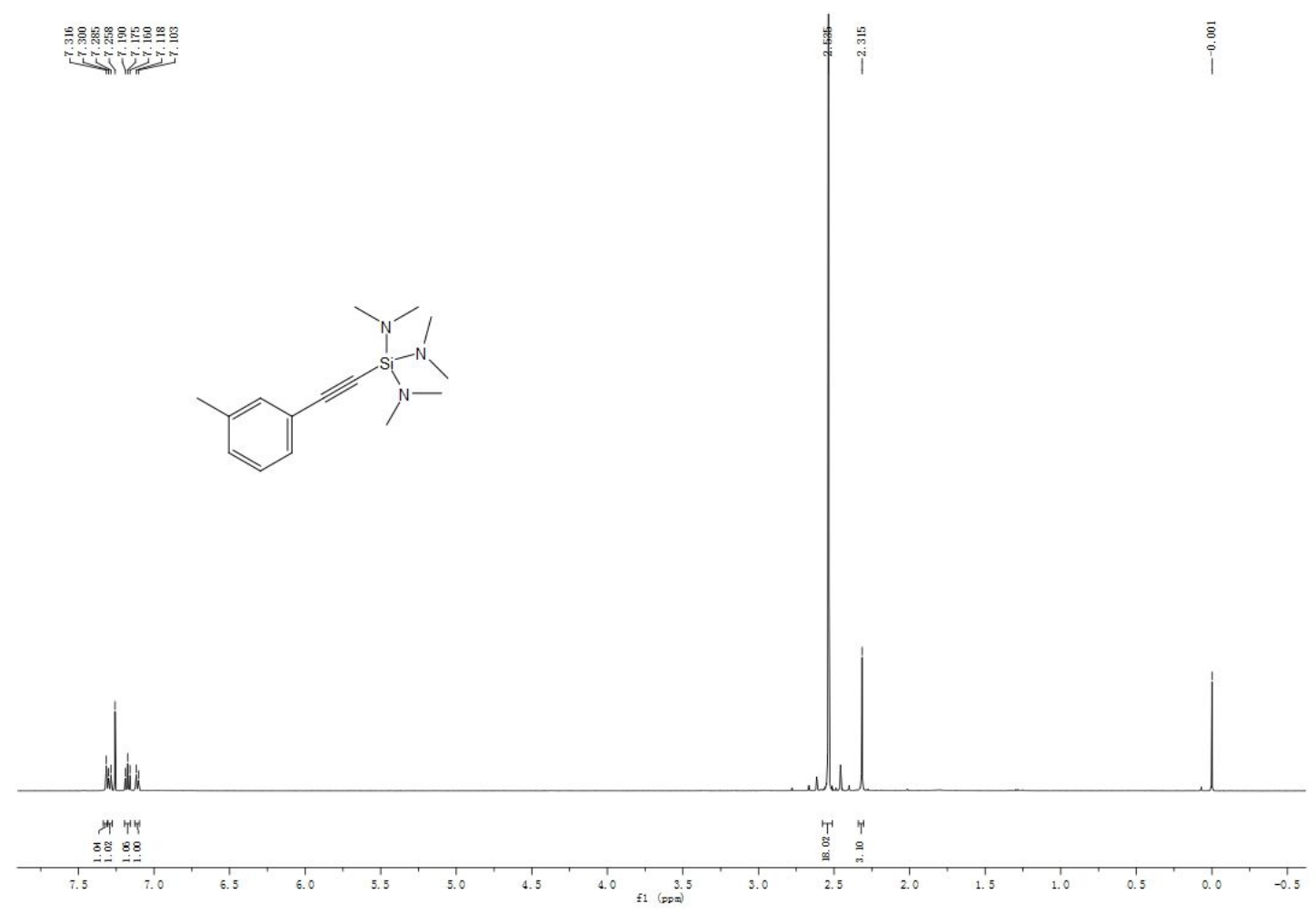

${ }^{1} \mathrm{H}$ NMR $\left(500 \mathrm{MHz}, \mathrm{CDCl}_{3}\right)$ spectrum of $4 \mathbf{c d}$

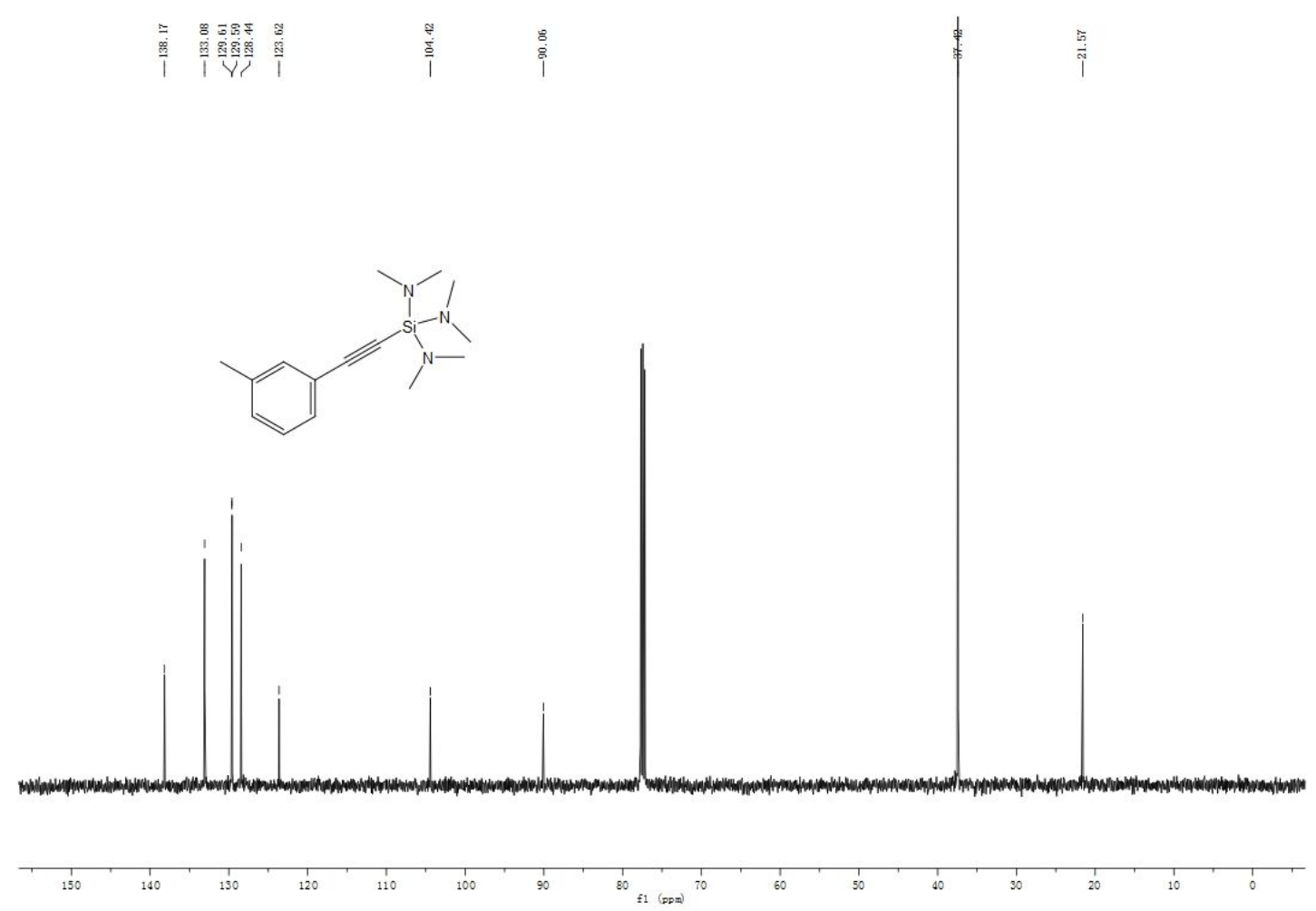

${ }^{13} \mathrm{C}$ NMR $\left(125 \mathrm{MHz}, \mathrm{CDCl}_{3}\right)$ spectrum of $\mathbf{4 c d}$ 


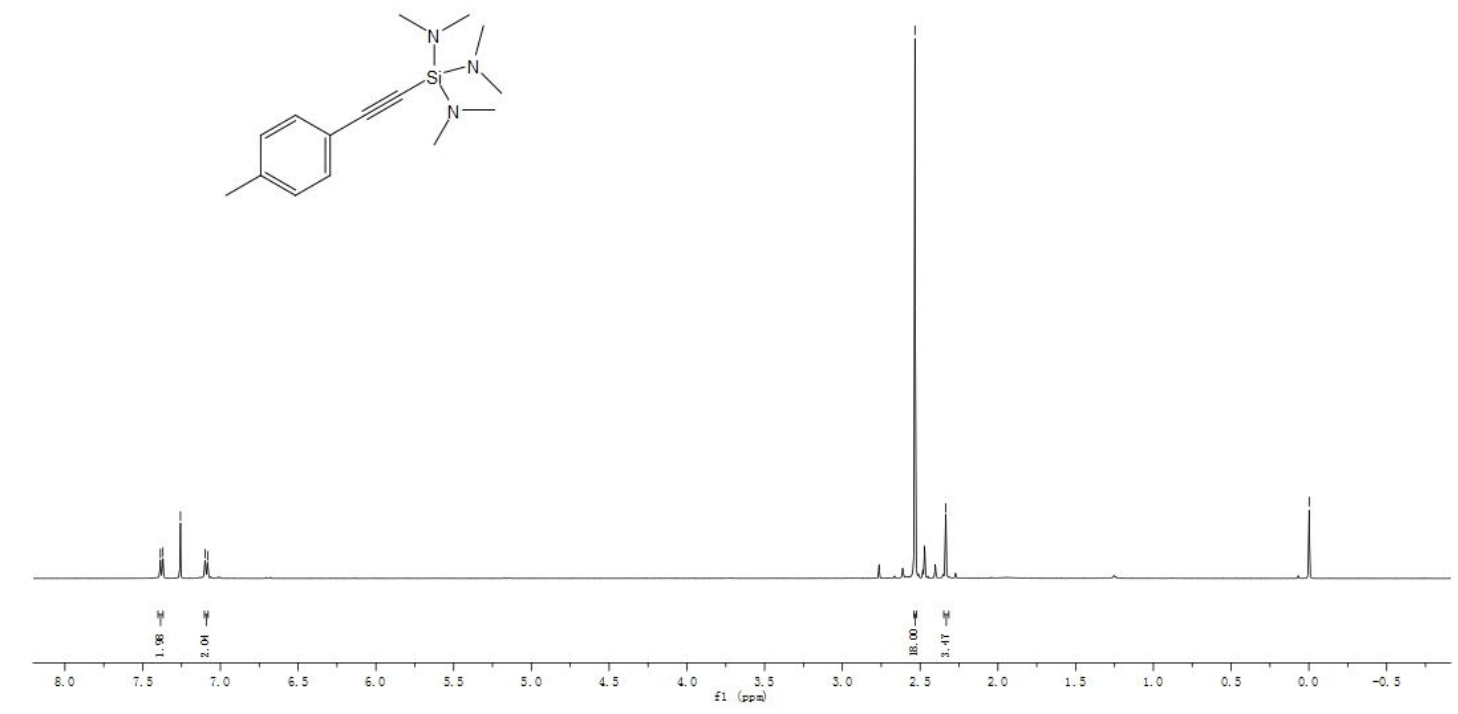

${ }^{1} \mathrm{H}$ NMR $\left(500 \mathrm{MHz}, \mathrm{CDCl}_{3}\right)$ spectrum of $\mathbf{4 d d}$

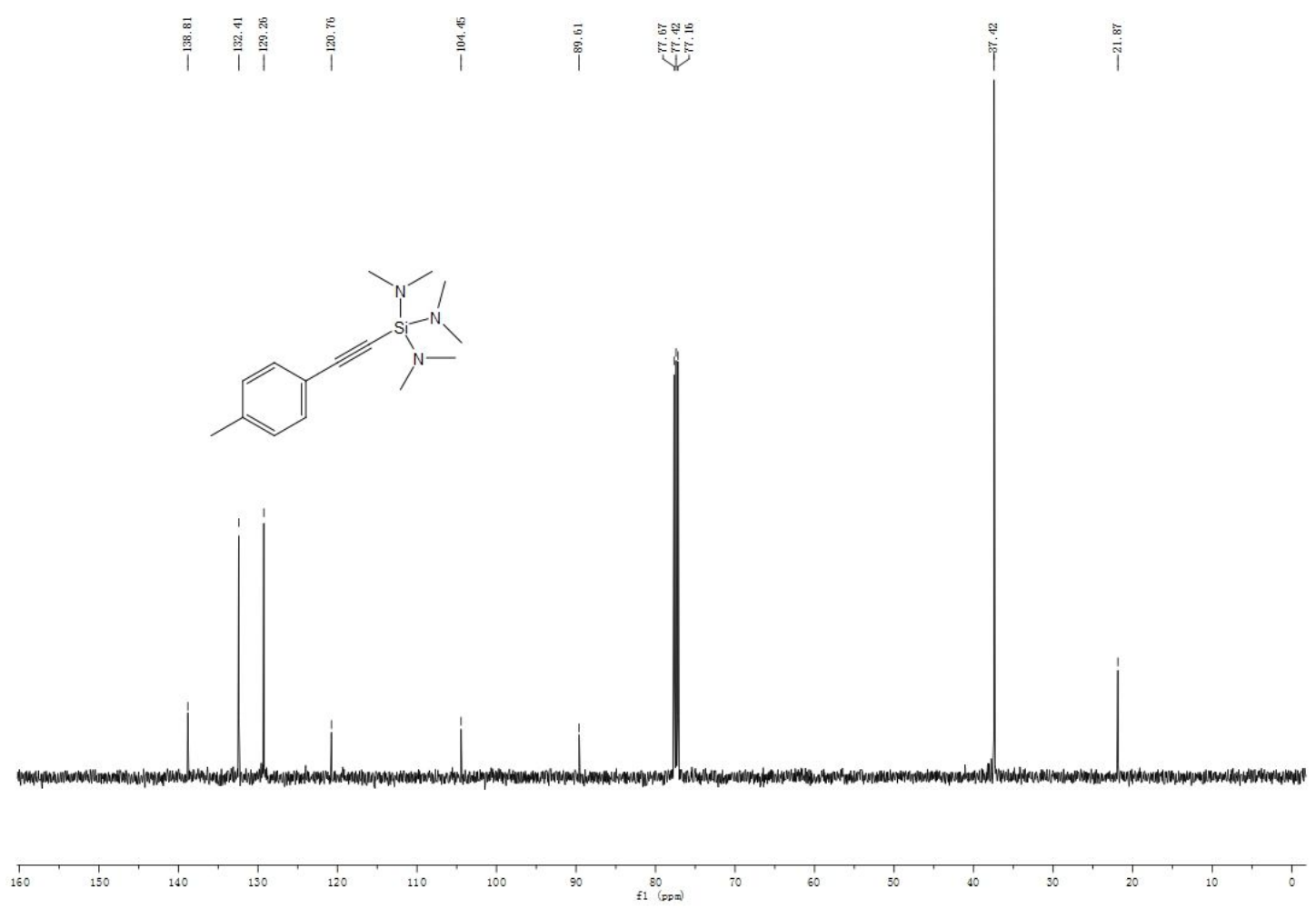

${ }^{13} \mathrm{C}$ NMR $\left(125 \mathrm{MHz}, \mathrm{CDCl}_{3}\right)$ spectrum of $\mathbf{4 d d}$ 


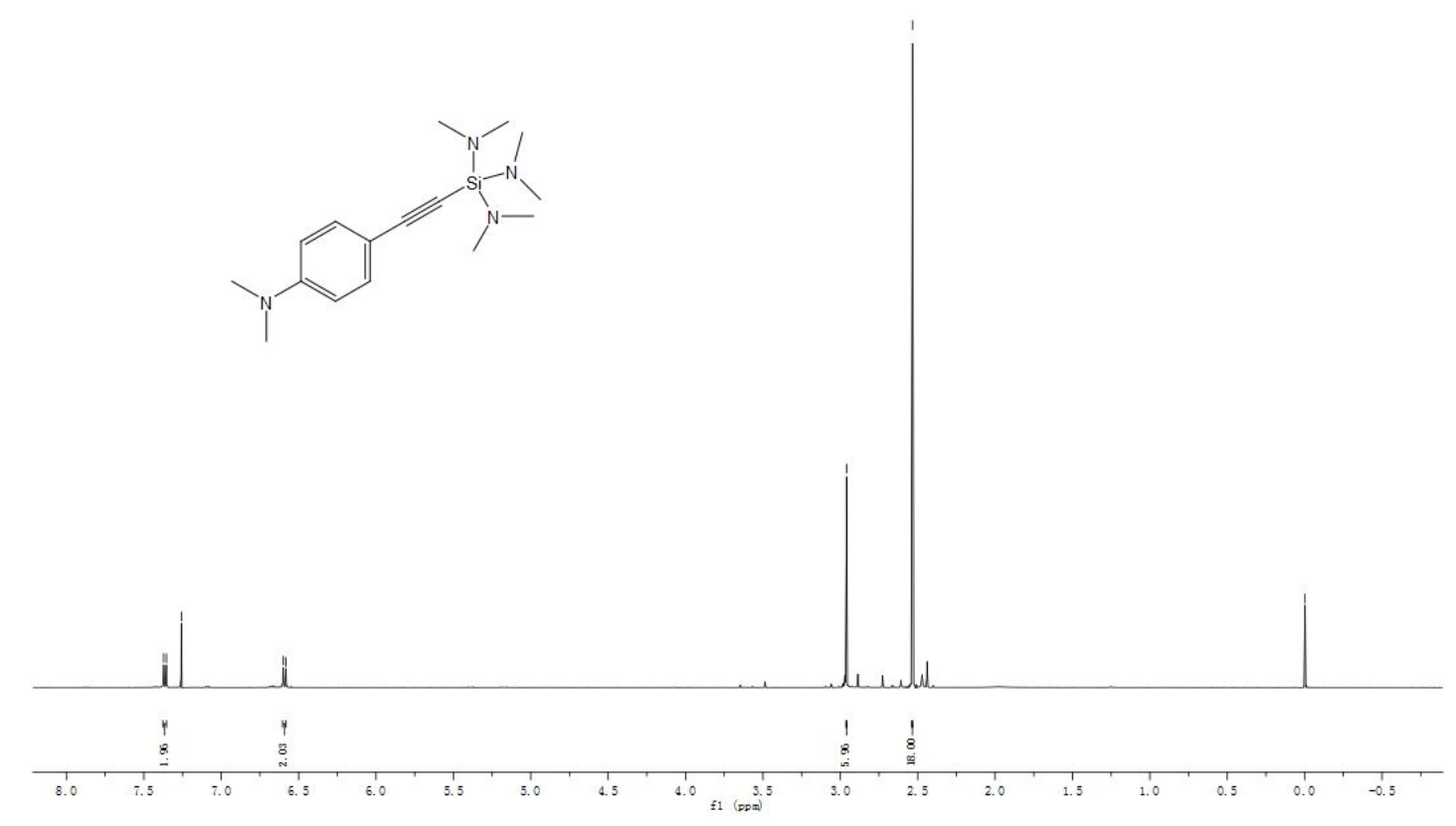

${ }^{1} \mathrm{H}$ NMR $\left(500 \mathrm{MHz}, \mathrm{CDCl}_{3}\right)$ spectrum of $\mathbf{4 h d}$

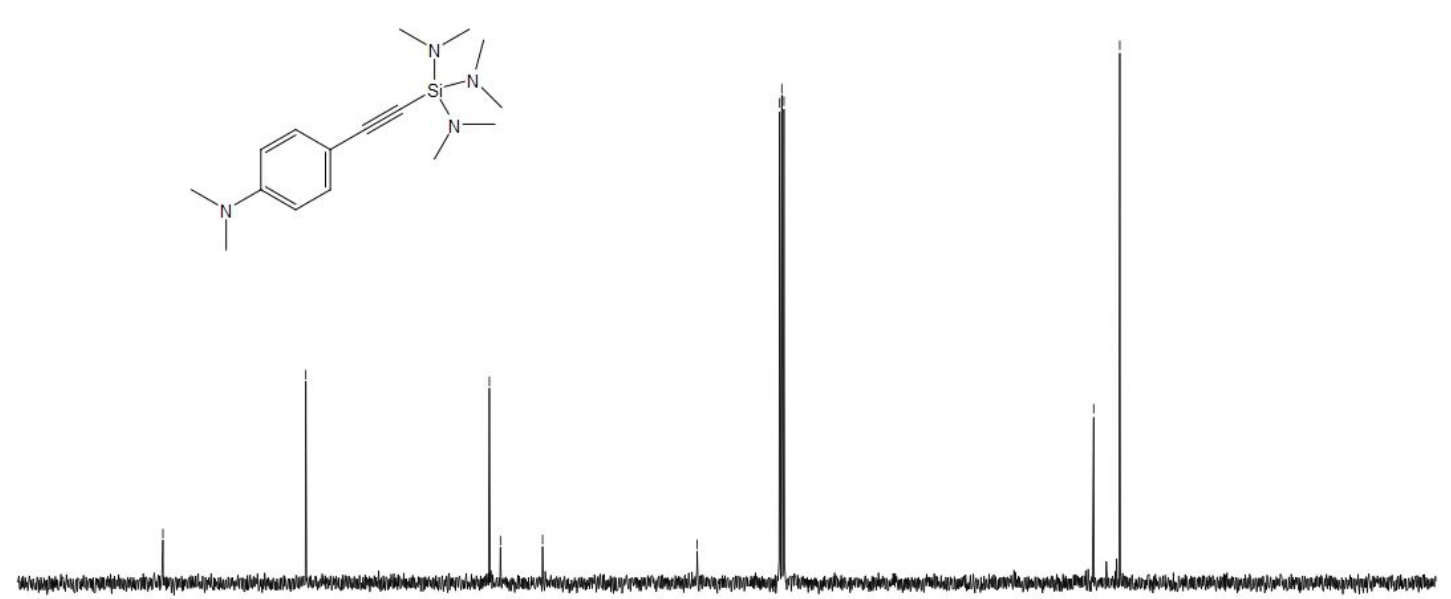

${ }^{13} \mathrm{C}$ NMR $\left(125 \mathrm{MHz}, \mathrm{CDCl}_{3}\right)$ spectrum of $\mathbf{4 h d}$ 


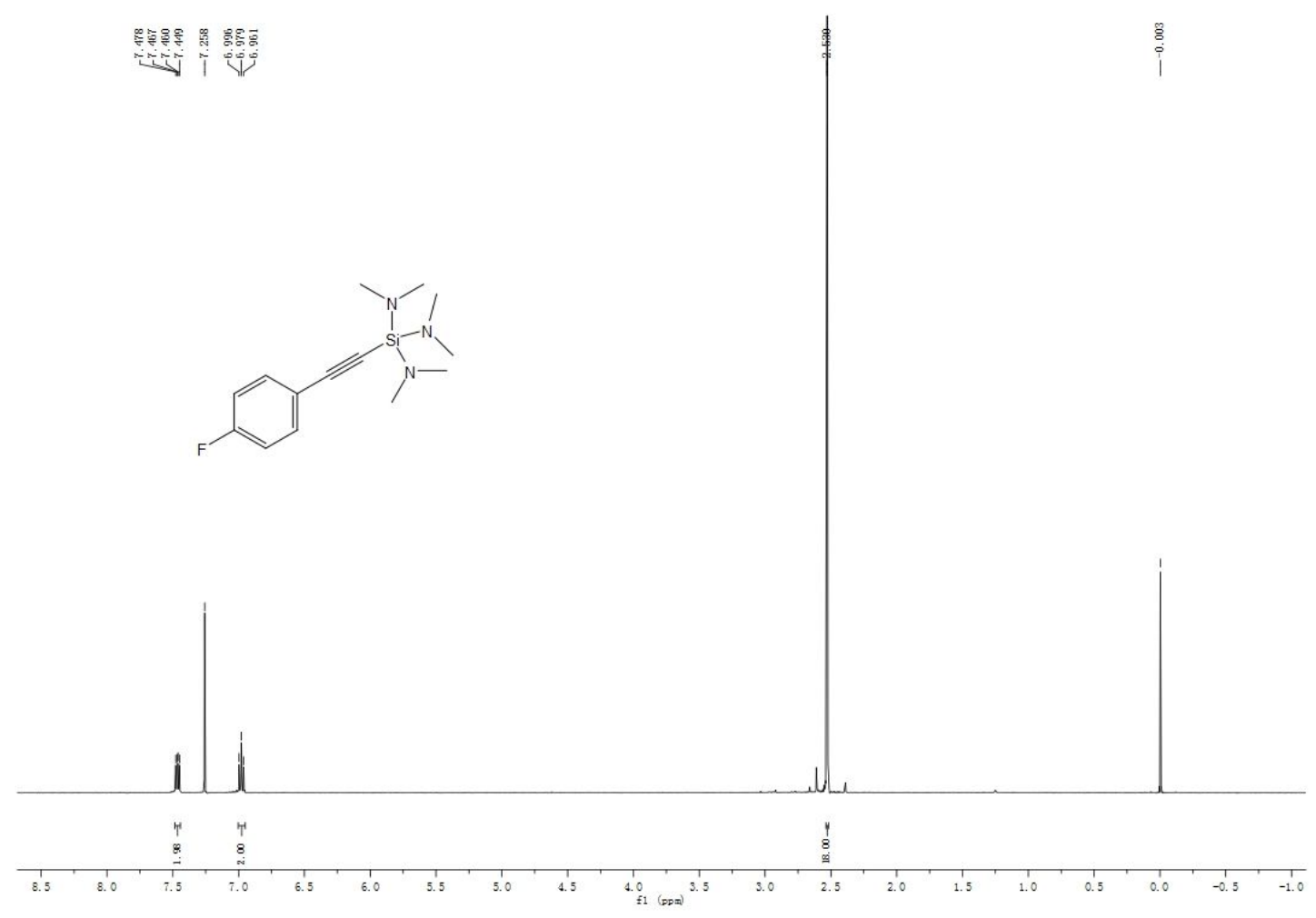

${ }^{1} \mathrm{H}$ NMR $\left(500 \mathrm{MHz}, \mathrm{CDCl}_{3}\right)$ spectrum of 4 id

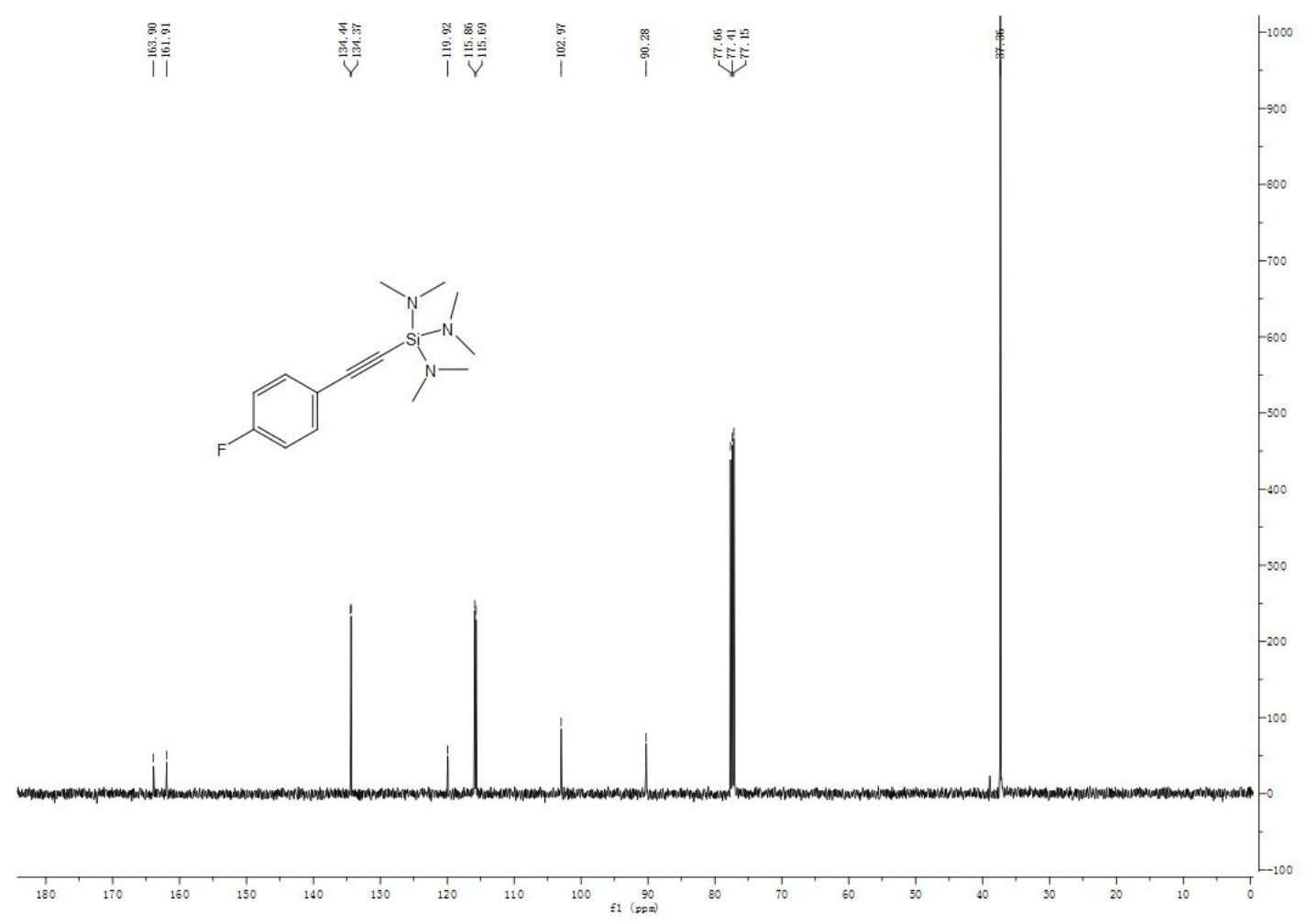

${ }^{13} \mathrm{C} \mathrm{NMR}\left(125 \mathrm{MHz}, \mathrm{CDCl}_{3}\right)$ spectrum of $4 \mathbf{i d}$ 


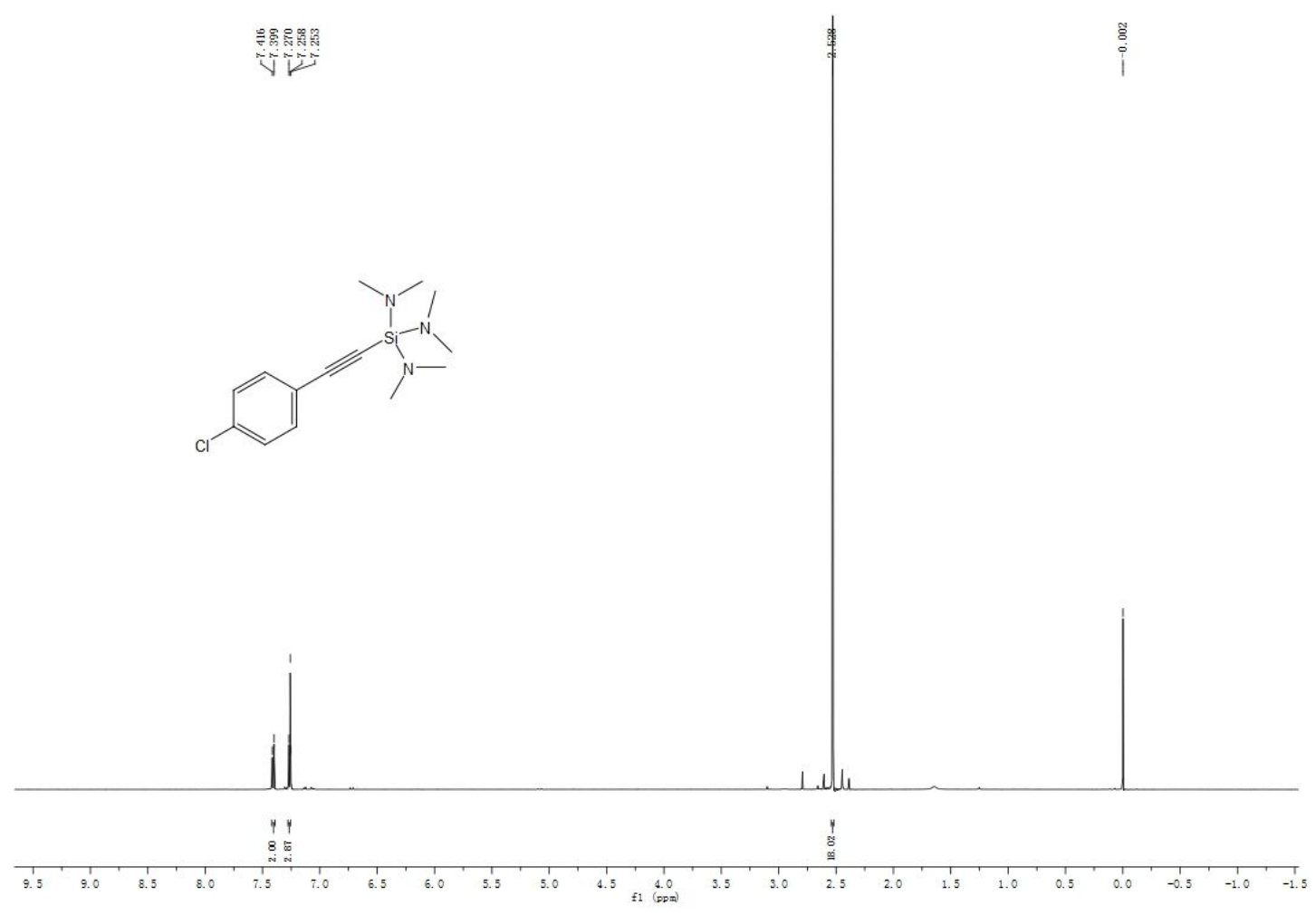

${ }^{1} \mathrm{H}$ NMR $\left(500 \mathrm{MHz}, \mathrm{CDCl}_{3}\right)$ spectrum of $\mathbf{4 j d}$
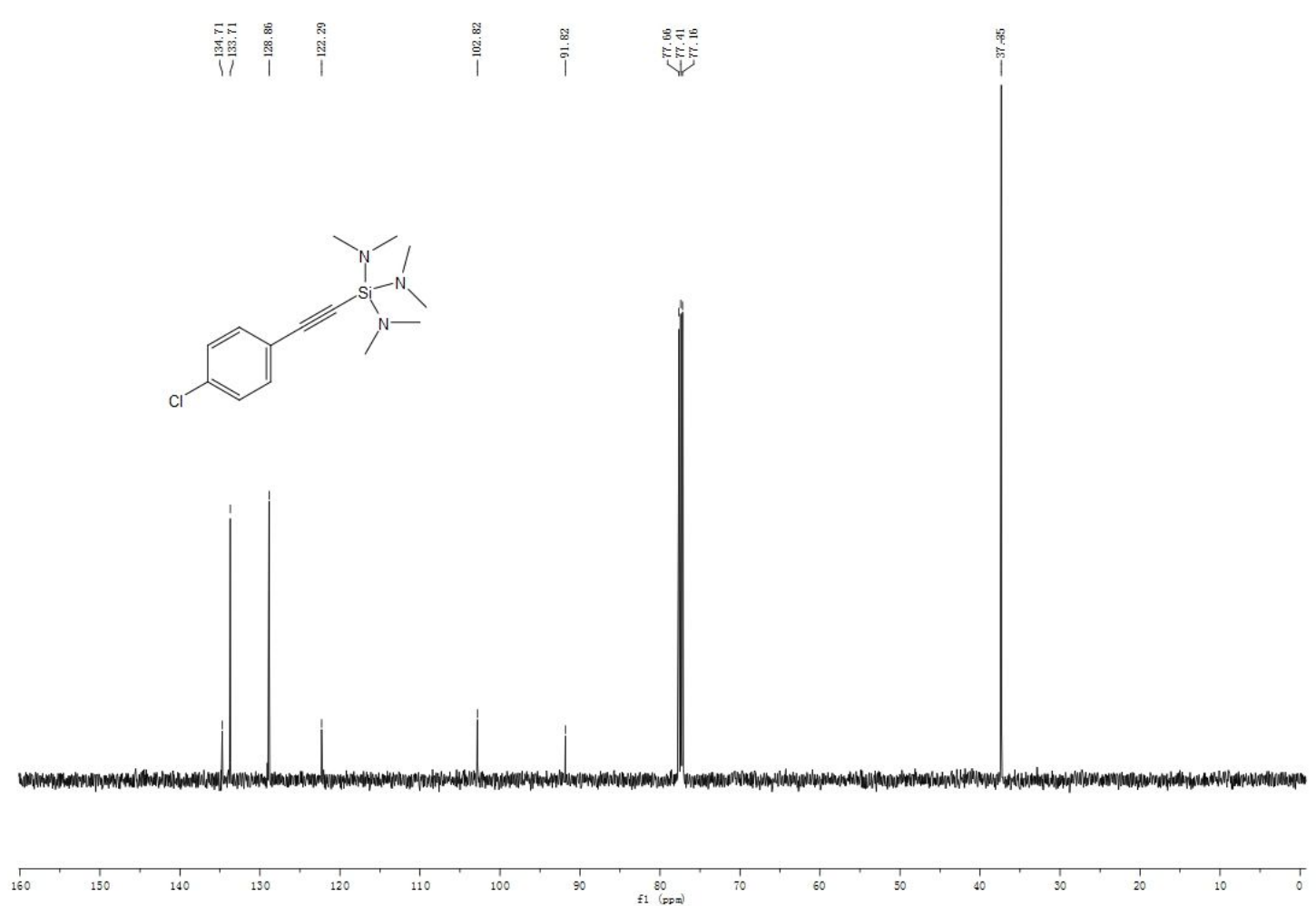

${ }^{13} \mathrm{C} \mathrm{NMR}\left(125 \mathrm{MHz}, \mathrm{CDCl}_{3}\right)$ spectrum of $\mathbf{4 j d}$ 


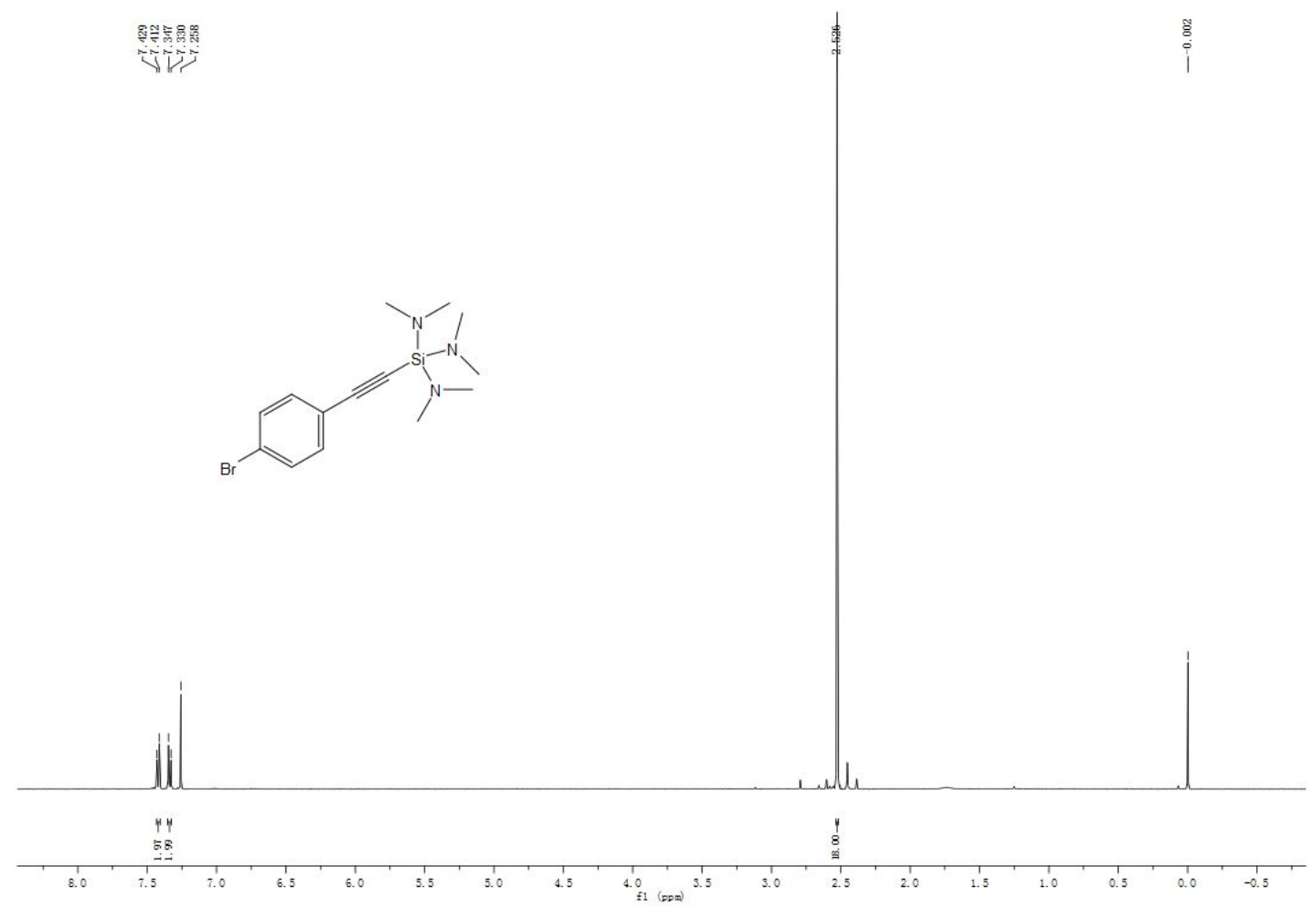

${ }^{1} \mathrm{H}$ NMR $\left(500 \mathrm{MHz}, \mathrm{CDCl}_{3}\right)$ spectrum of $\mathbf{4 k d}$
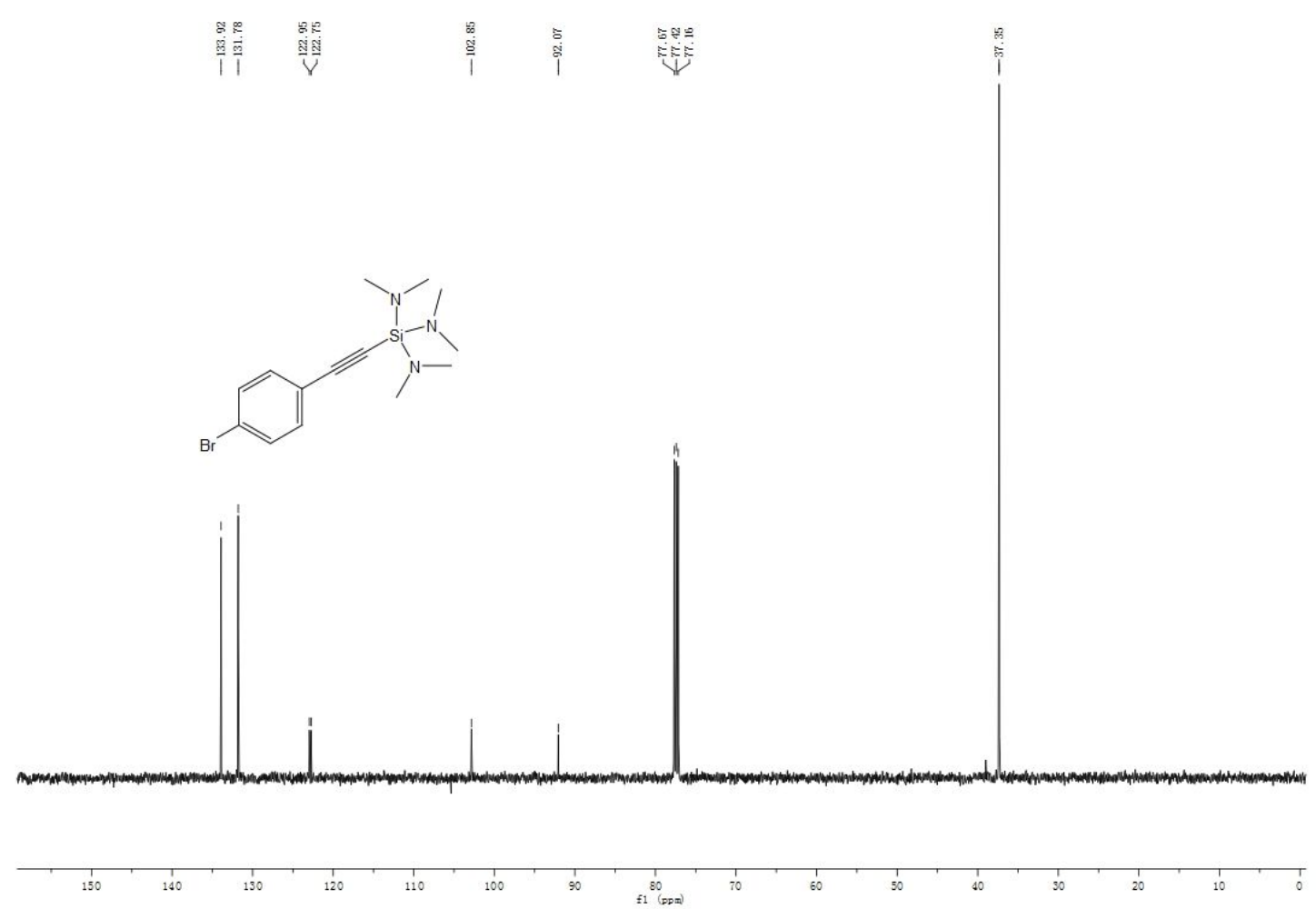

${ }^{13} \mathrm{C}$ NMR $\left(125 \mathrm{MHz}, \mathrm{CDCl}_{3}\right)$ spectrum of $\mathbf{4 k d}$ 


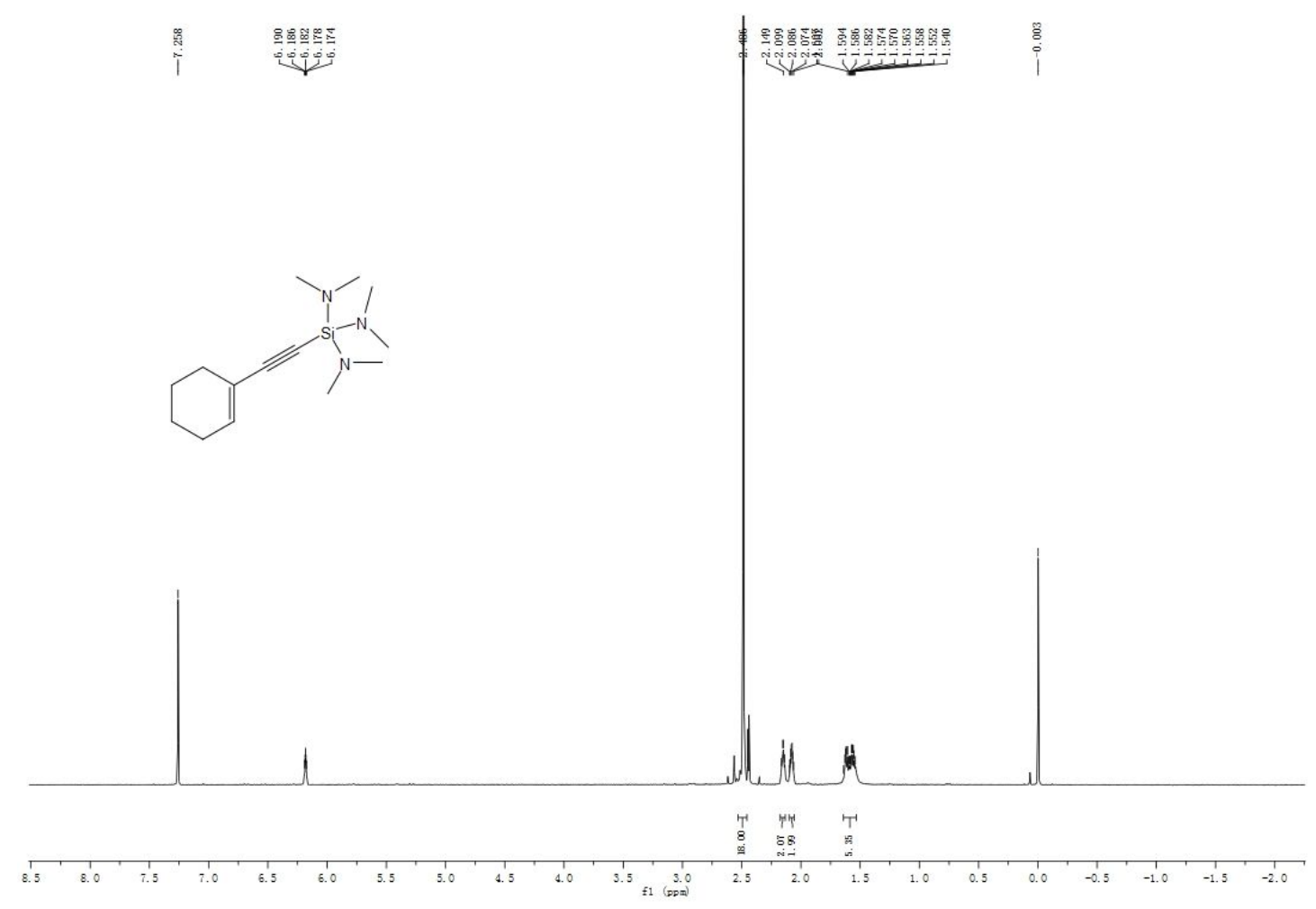

${ }^{1} \mathrm{H}$ NMR $\left(500 \mathrm{MHz}, \mathrm{CDCl}_{3}\right)$ spectrum of $\mathbf{4} \mathbf{m d}$
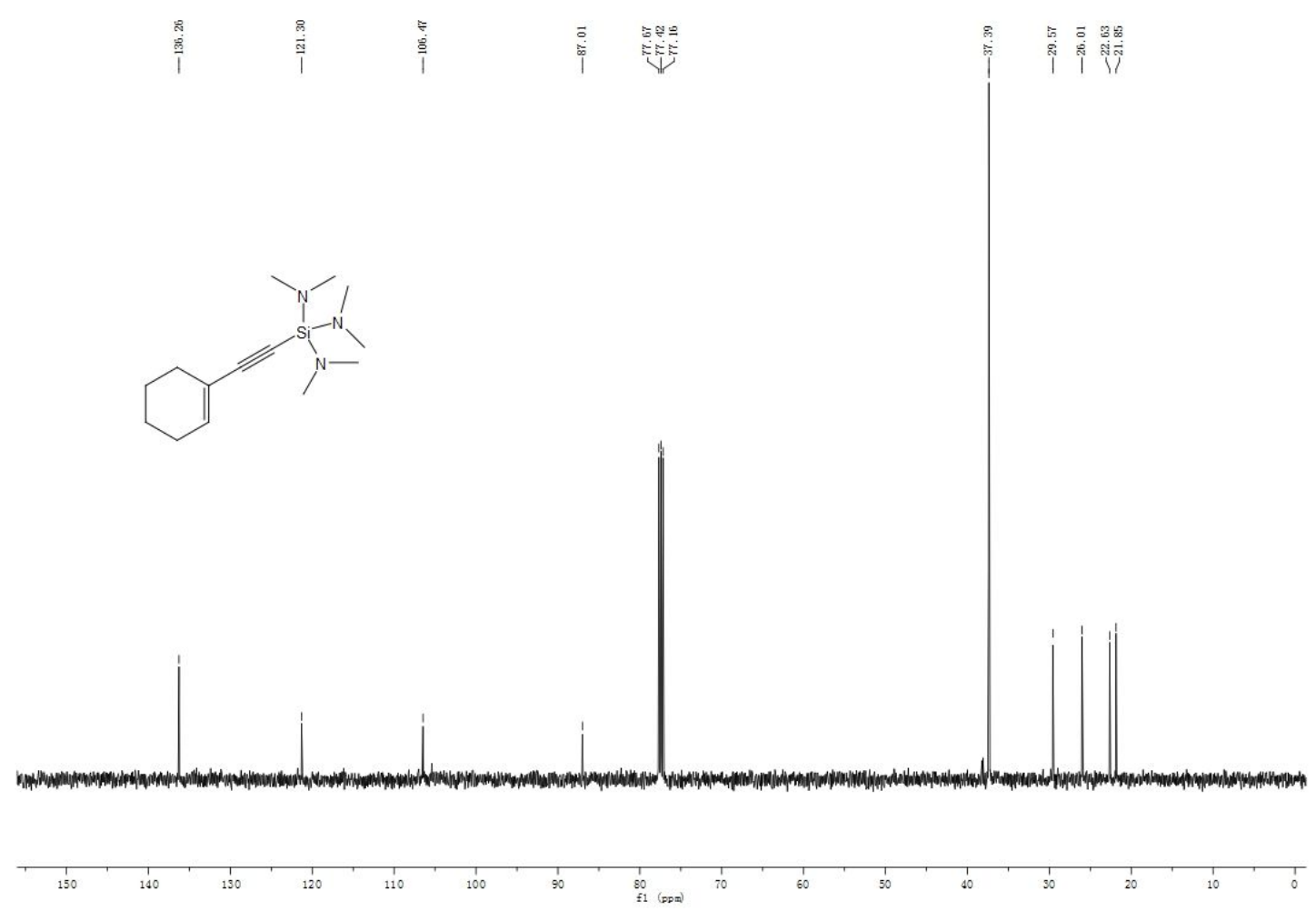

${ }^{13} \mathrm{C}$ NMR $\left(125 \mathrm{MHz}, \mathrm{CDCl}_{3}\right)$ spectrum of $\mathbf{4} \mathbf{m d}$ 


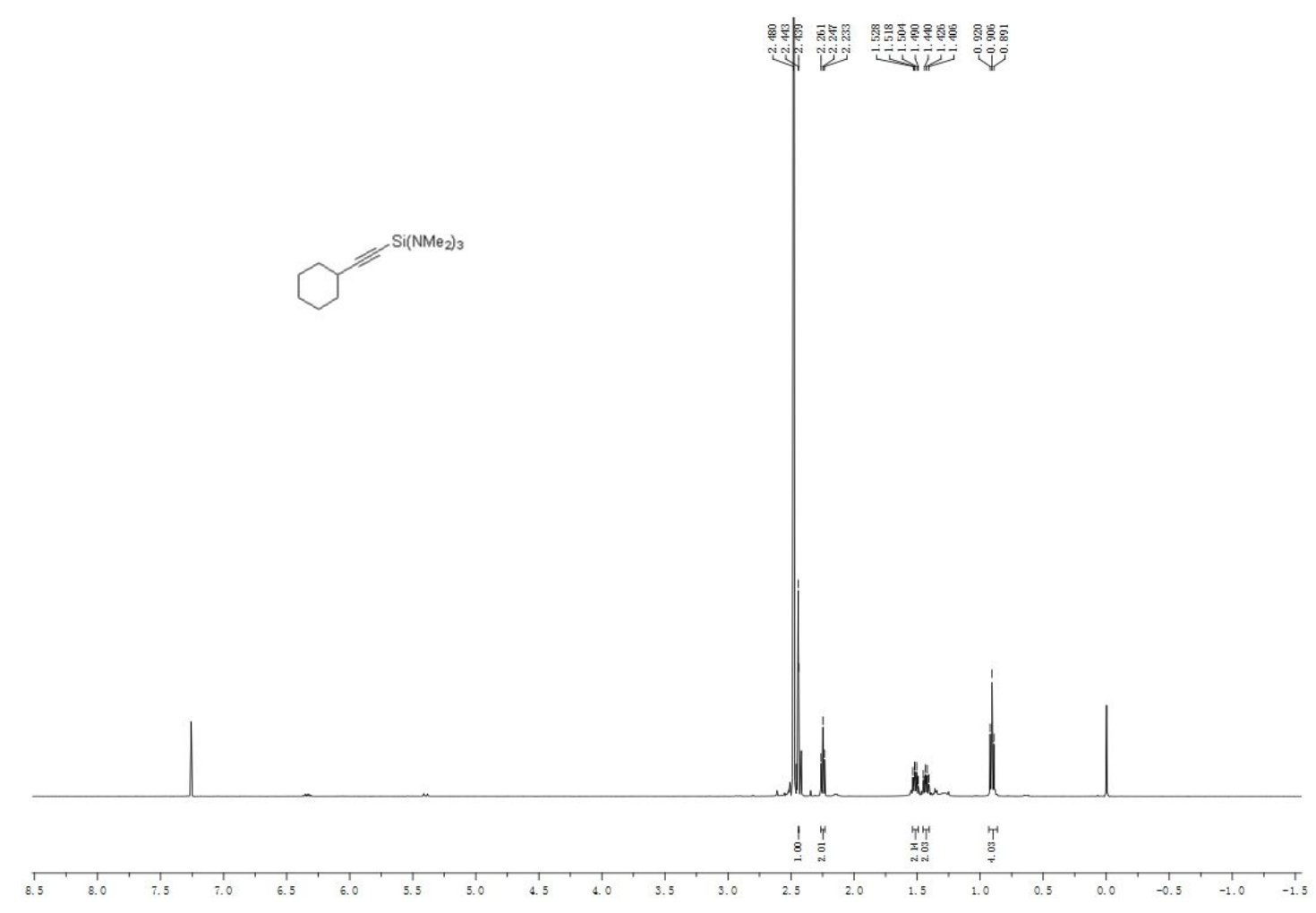

${ }^{1} \mathrm{H}$ NMR $\left(500 \mathrm{MHz}, \mathrm{CDCl}_{3}\right)$ spectrum of 4 nd
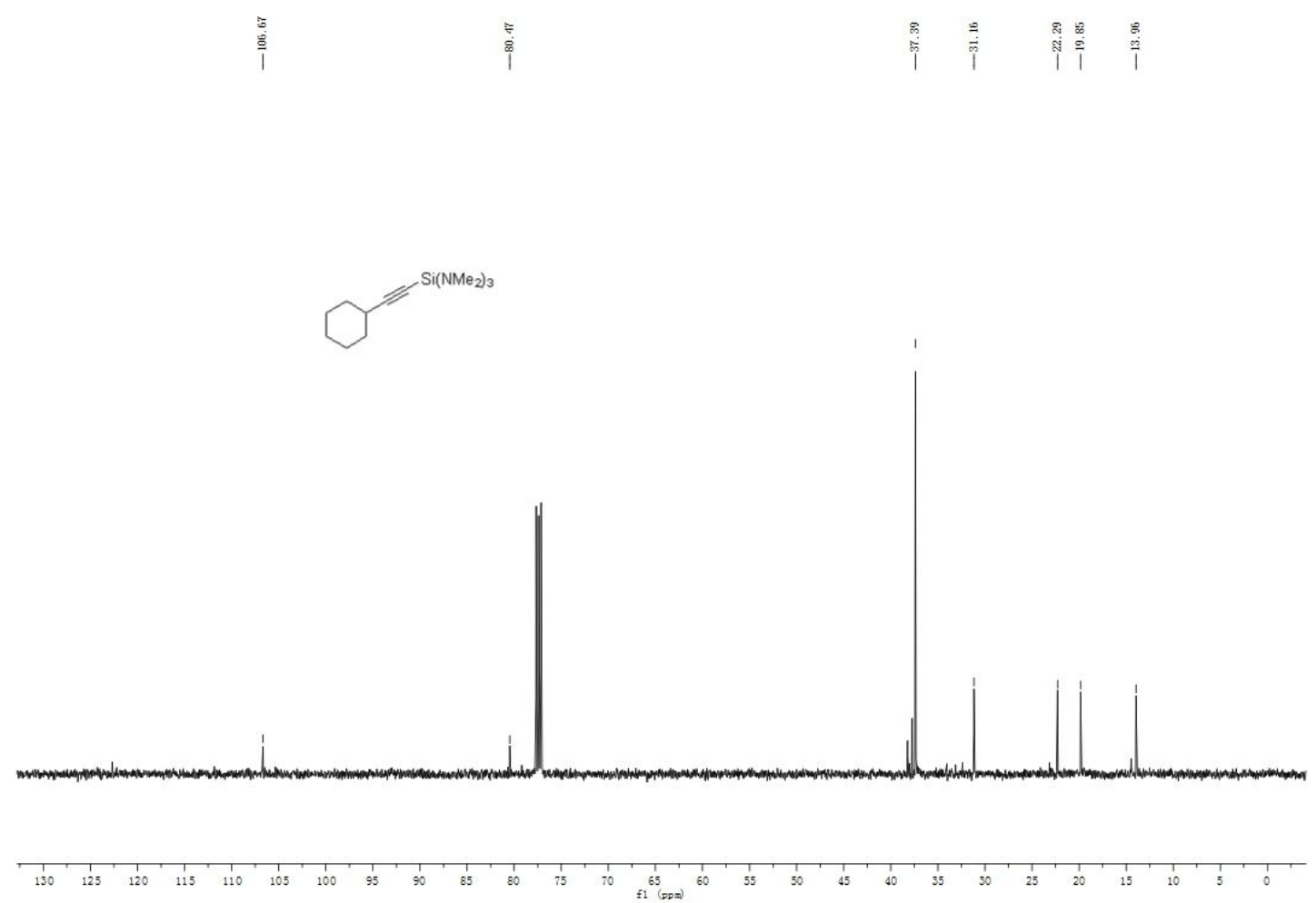

${ }^{13} \mathrm{C} \mathrm{NMR}\left(125 \mathrm{MHz}, \mathrm{CDCl}_{3}\right)$ spectrum of 4 nd 


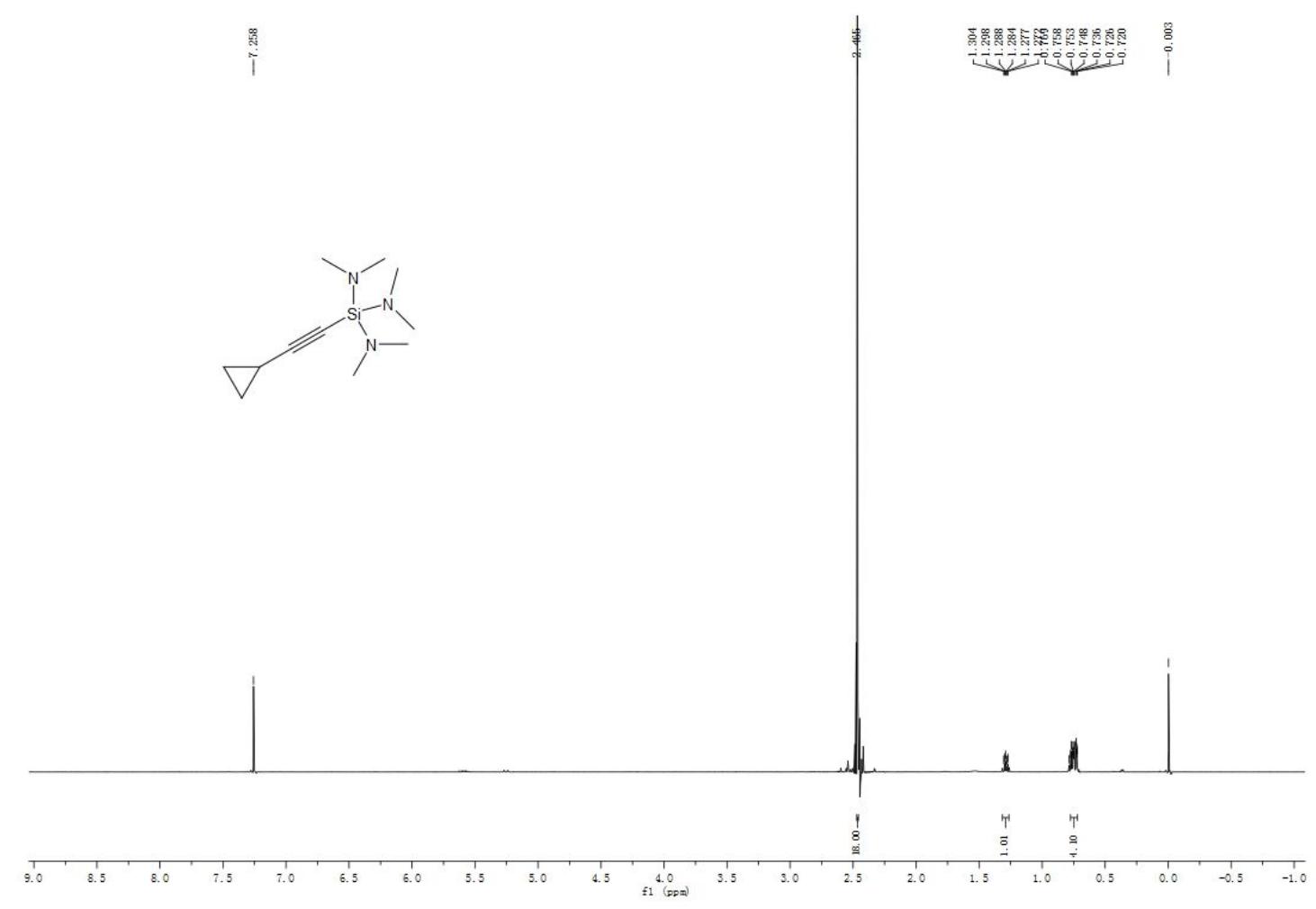

${ }^{1} \mathrm{H}$ NMR $\left(500 \mathrm{MHz}, \mathrm{CDCl}_{3}\right)$ spectrum of $\mathbf{4 o d}$
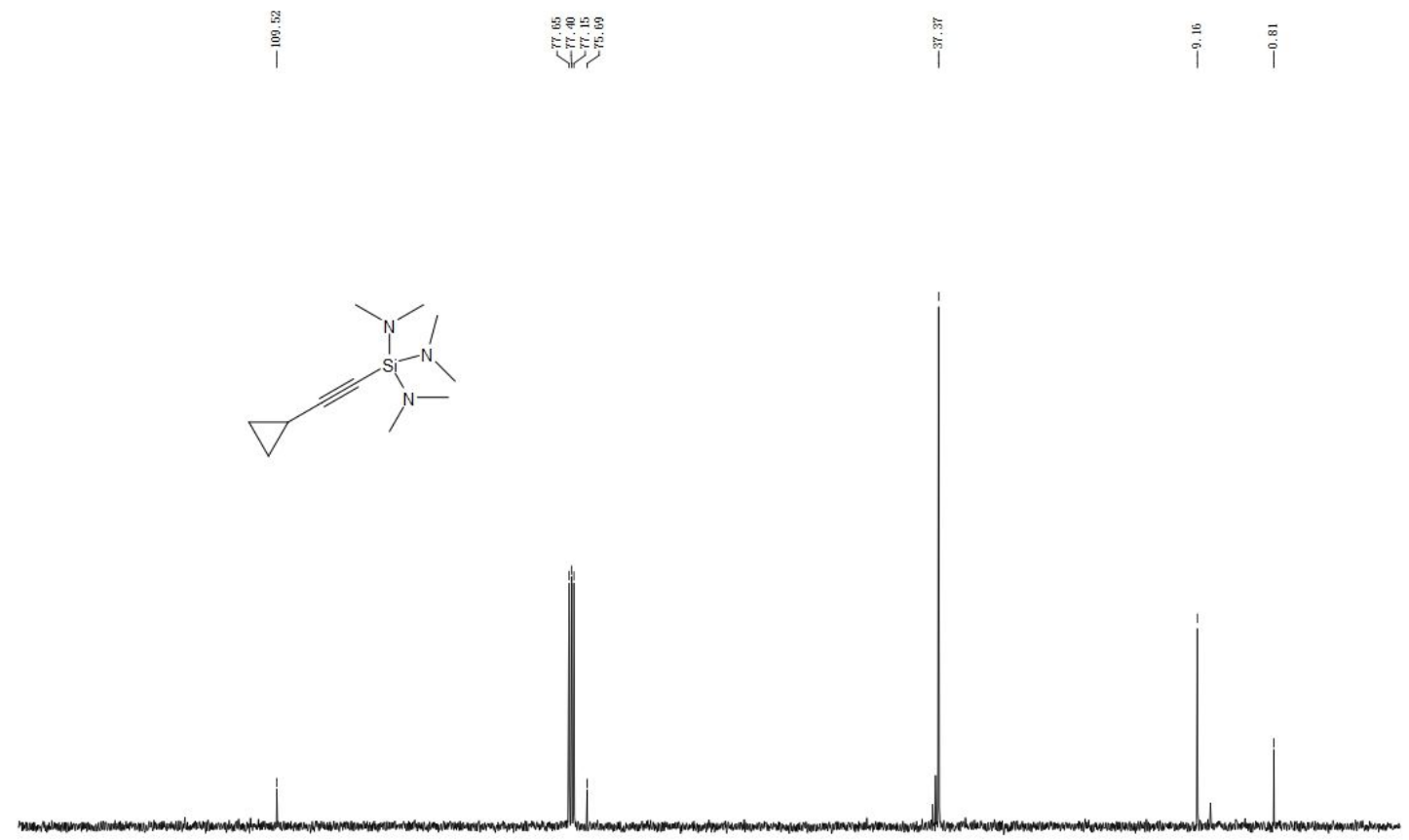

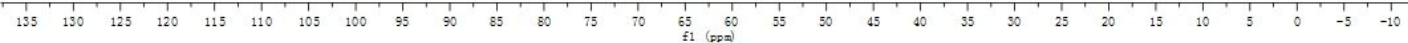

${ }^{13} \mathrm{C}$ NMR $\left(125 \mathrm{MHz}, \mathrm{CDCl}_{3}\right)$ spectrum of $4 \mathbf{o d}$ 


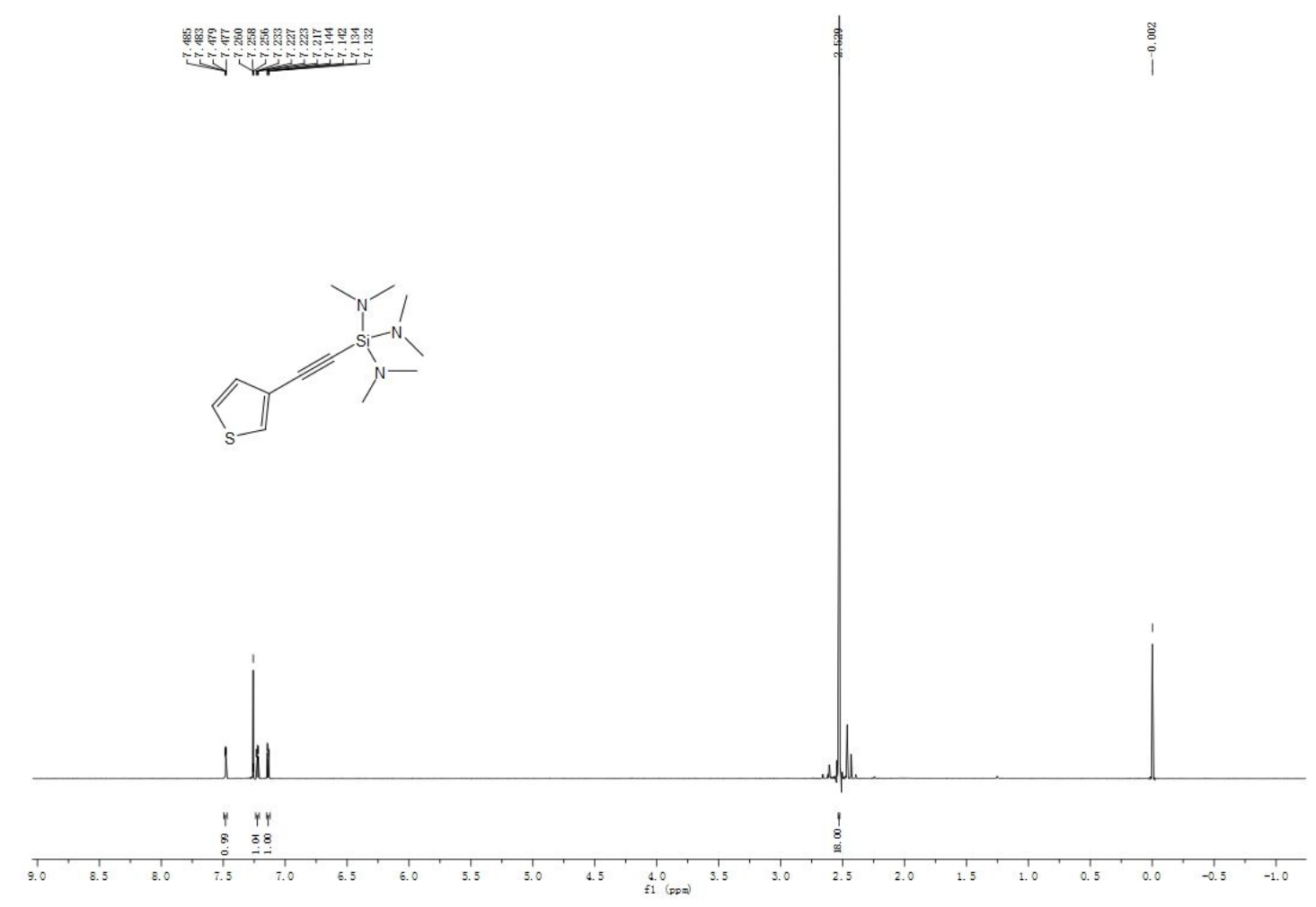

${ }^{1} \mathrm{H}$ NMR $\left(500 \mathrm{MHz}, \mathrm{CDCl}_{3}\right)$ spectrum of $\mathbf{4 p d}$

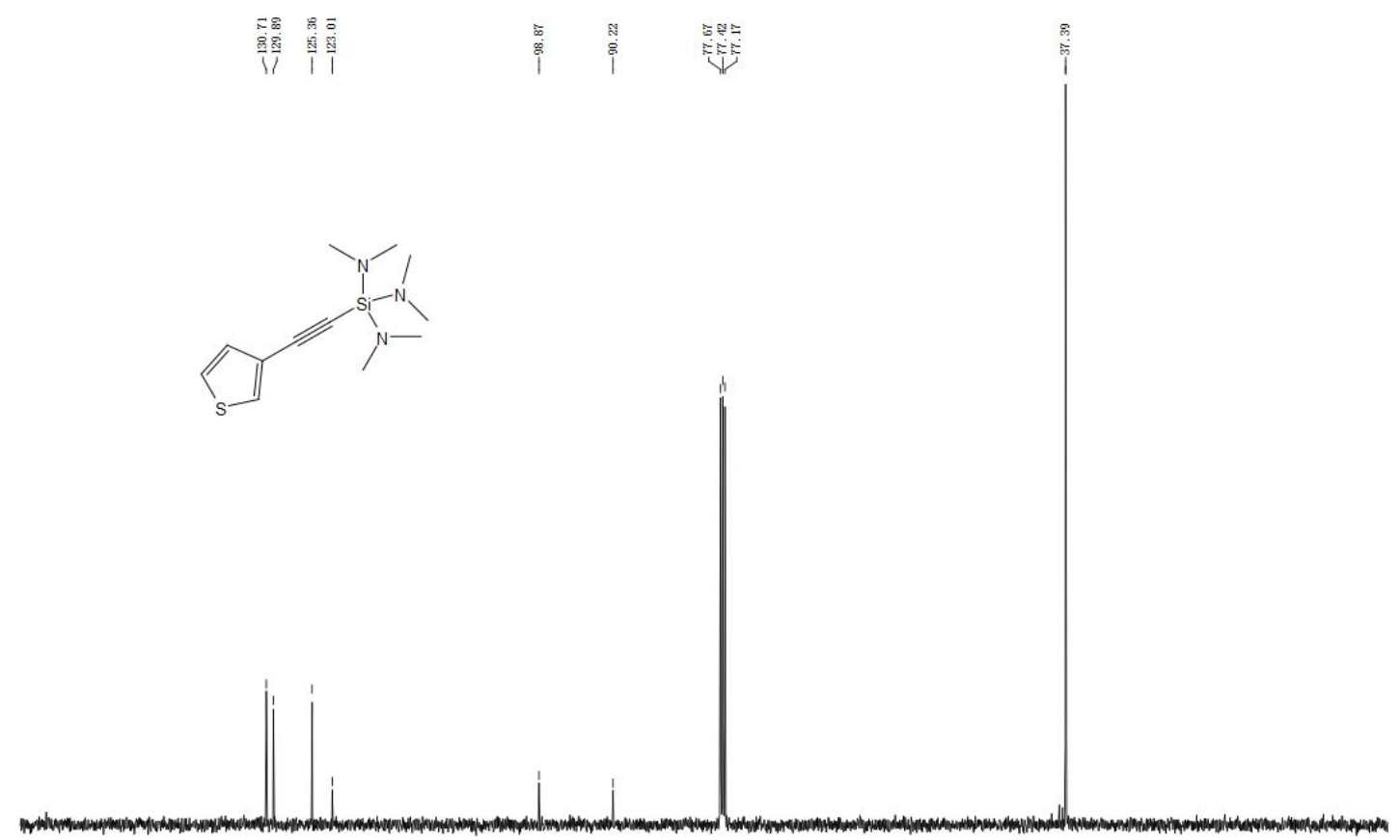

${ }^{13} \mathrm{C}$ NMR $\left(125 \mathrm{MHz}, \mathrm{CDCl}_{3}\right)$ spectrum of 4 pd 


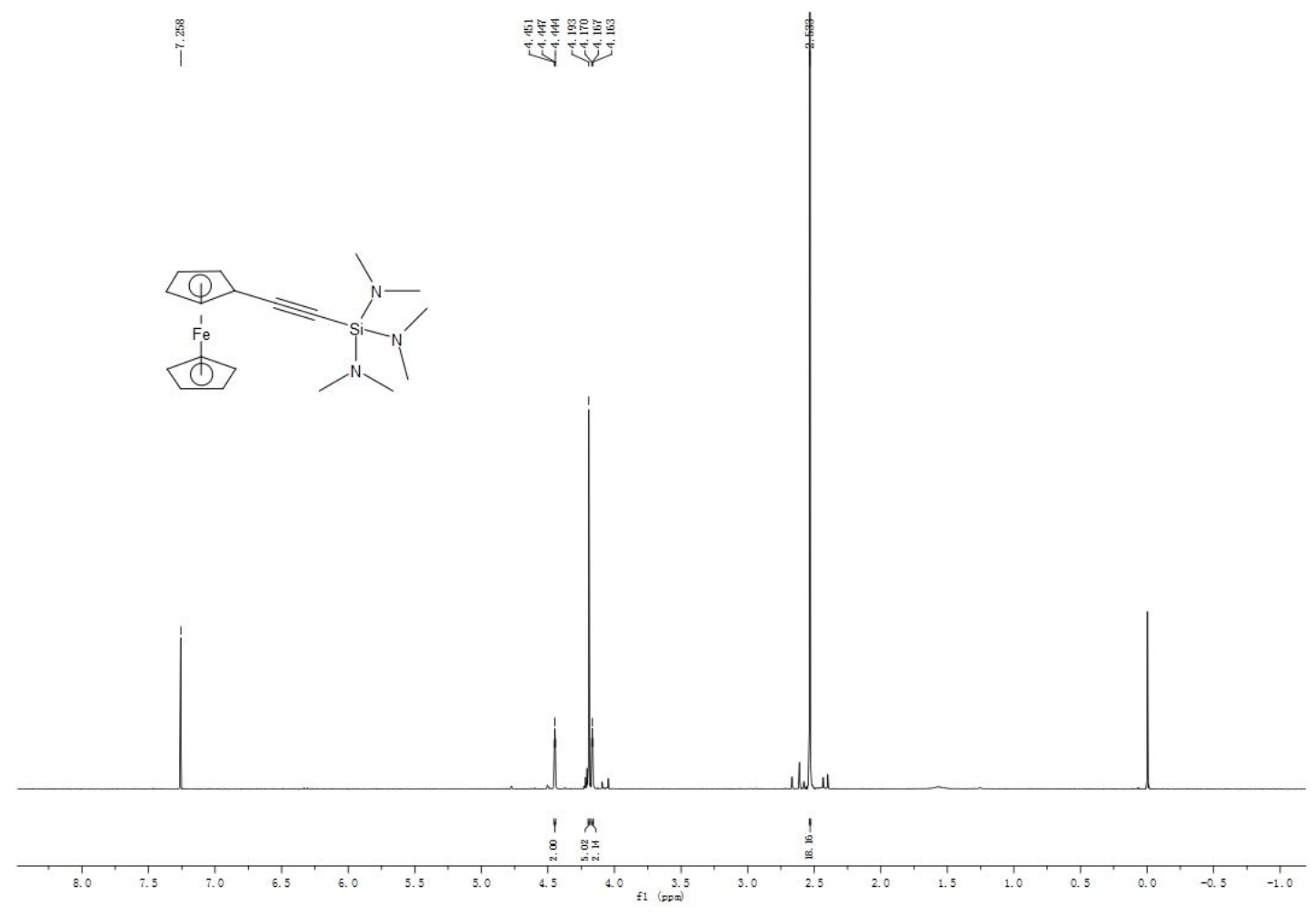

${ }^{1} \mathrm{H}$ NMR $\left(500 \mathrm{MHz}, \mathrm{CDCl}_{3}\right)$ spectrum of $\mathbf{4 q d}$
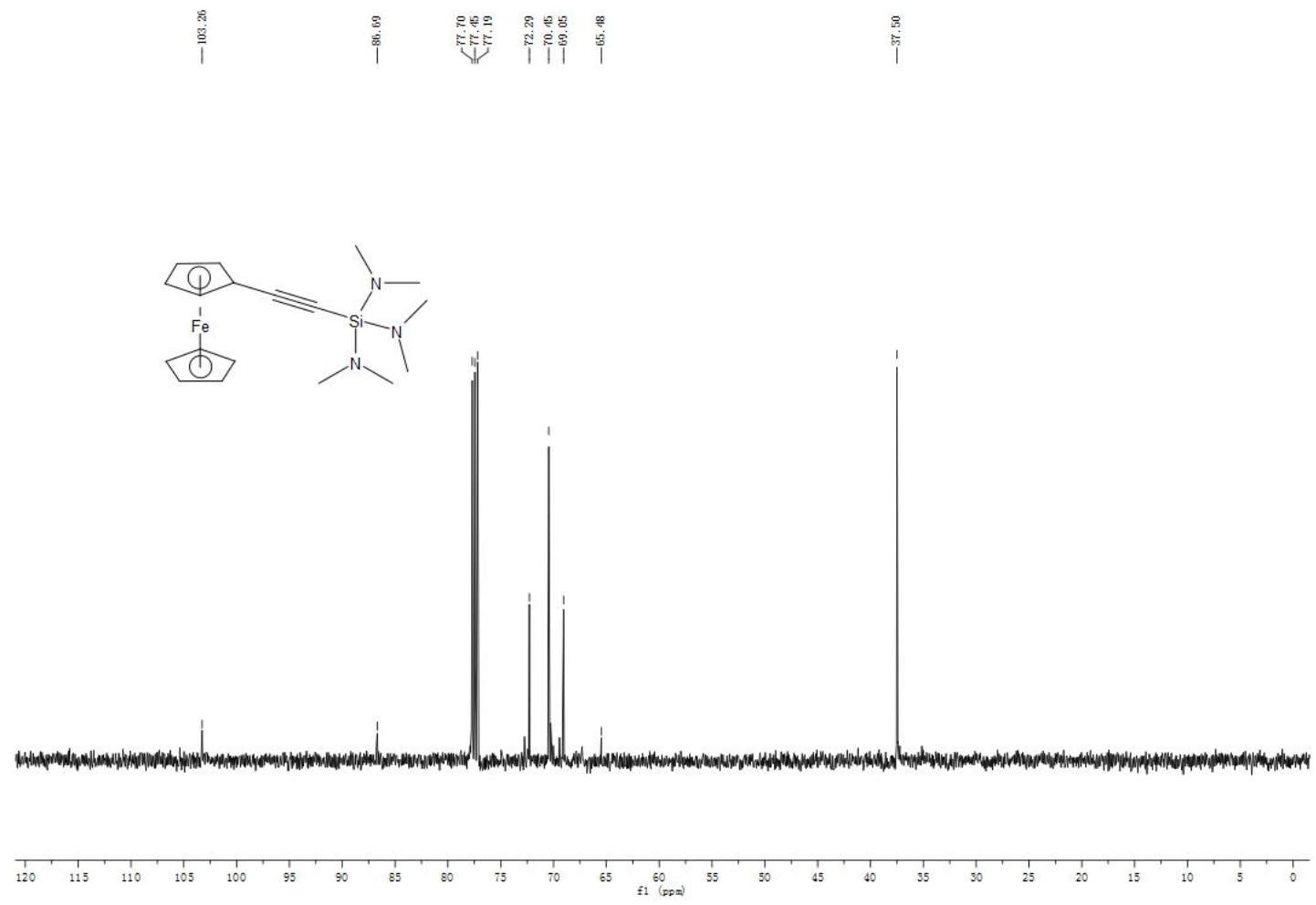

${ }^{13} \mathrm{C}$ NMR $\left(125 \mathrm{MHz}, \mathrm{CDCl}_{3}\right)$ spectrum of $\mathbf{4 q d}$ 


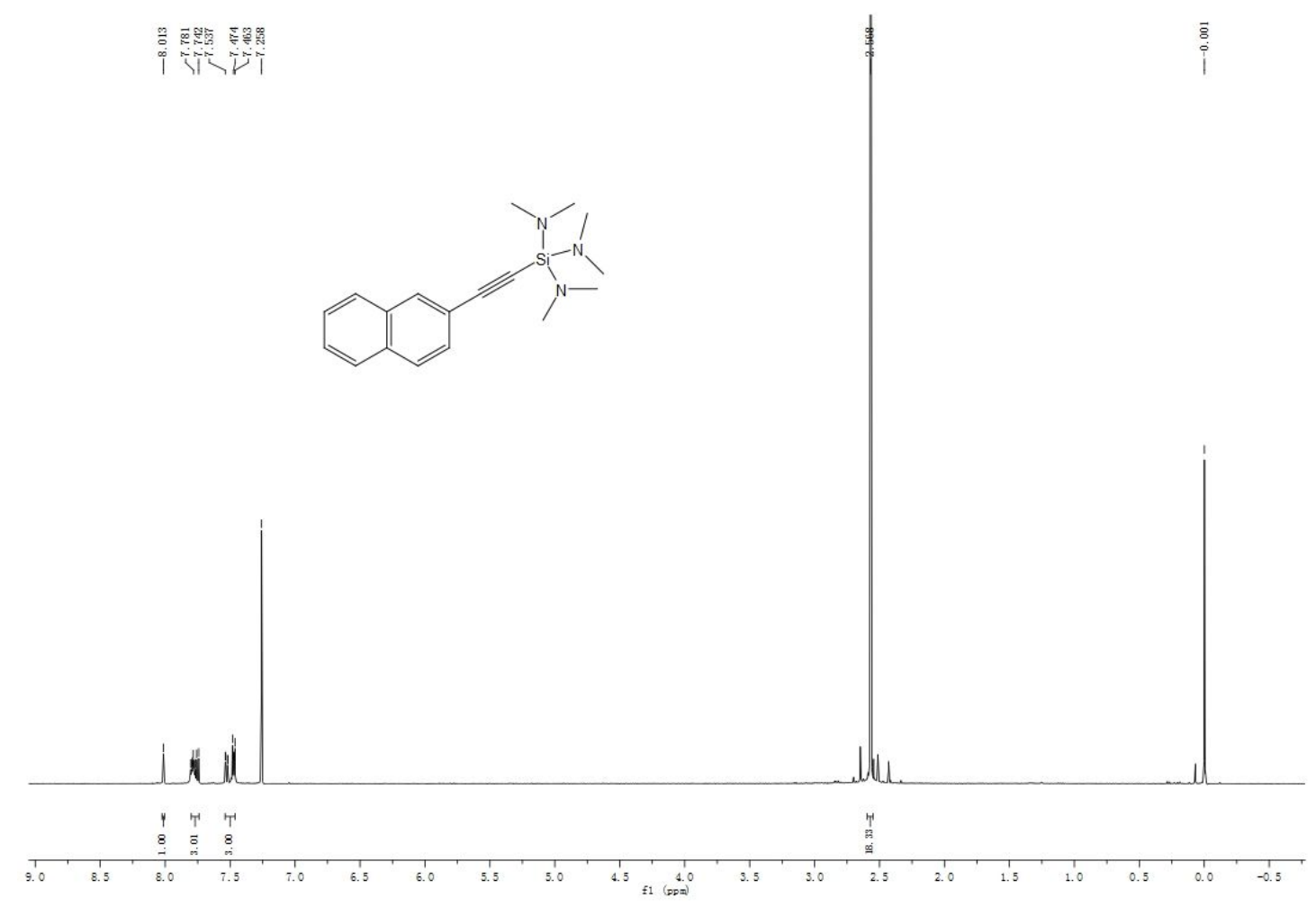

${ }^{1} \mathrm{H}$ NMR (500 MHz, $\mathrm{CDCl}_{3}$ ) spectrum of $4 \mathbf{s d}$

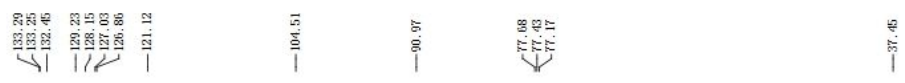
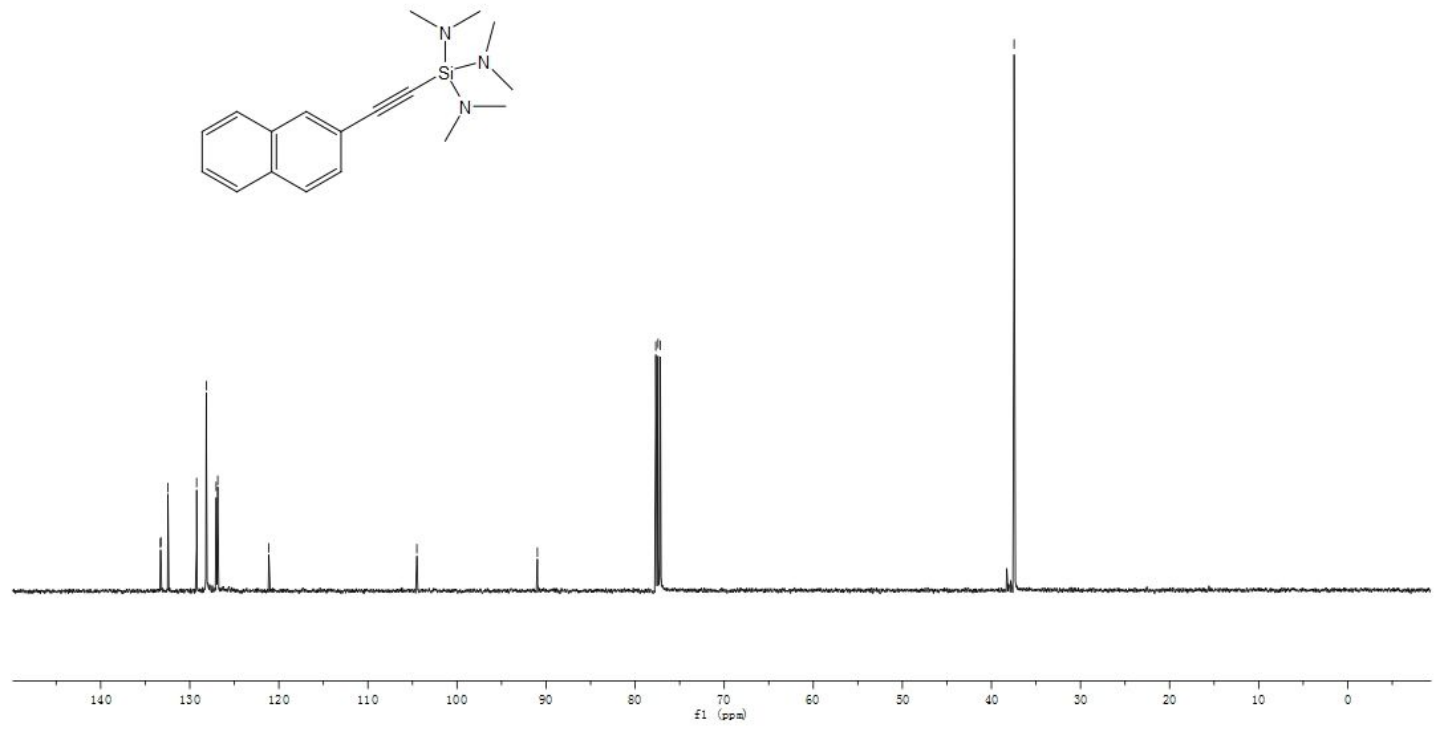

${ }^{13} \mathrm{C}$ NMR $\left(125 \mathrm{MHz}, \mathrm{CDCl}_{3}\right)$ spectrum of $4 \mathbf{s d}$ 


\section{DFT calculations}

All the electronic structure calculations were performed using the SIESTA package with Troullier-Martins norm conserving pseudopotentials. ${ }^{4-6}$ The exchangecorrelation functional utilized was at the generalized gradient approximation (GGA) level with Perdew-Burke-Ernzerhof (PBE). ${ }^{7}$ A double- $\xi$ plus polarization (DZP) basis set was employed and the orbital-confirming cutoff was determined from an energy shift of $0.01 \mathrm{eV}$. The van der Waals interactions were considered by vdw-DF functional proposed by Dion et al. ${ }^{8}$ All atoms were allowed to move during optimizations. The cut-off energy for the real space grid was $300 \mathrm{Ry}$. The molecules were placed in a cubic box with the length of $35 \AA$. gamma Monkhorst-Pack $k$-point mesh was used. The transition states were located by a constrained optimization approach with the force converge criteria below $0.05 \mathrm{eV} / \AA .^{9}$

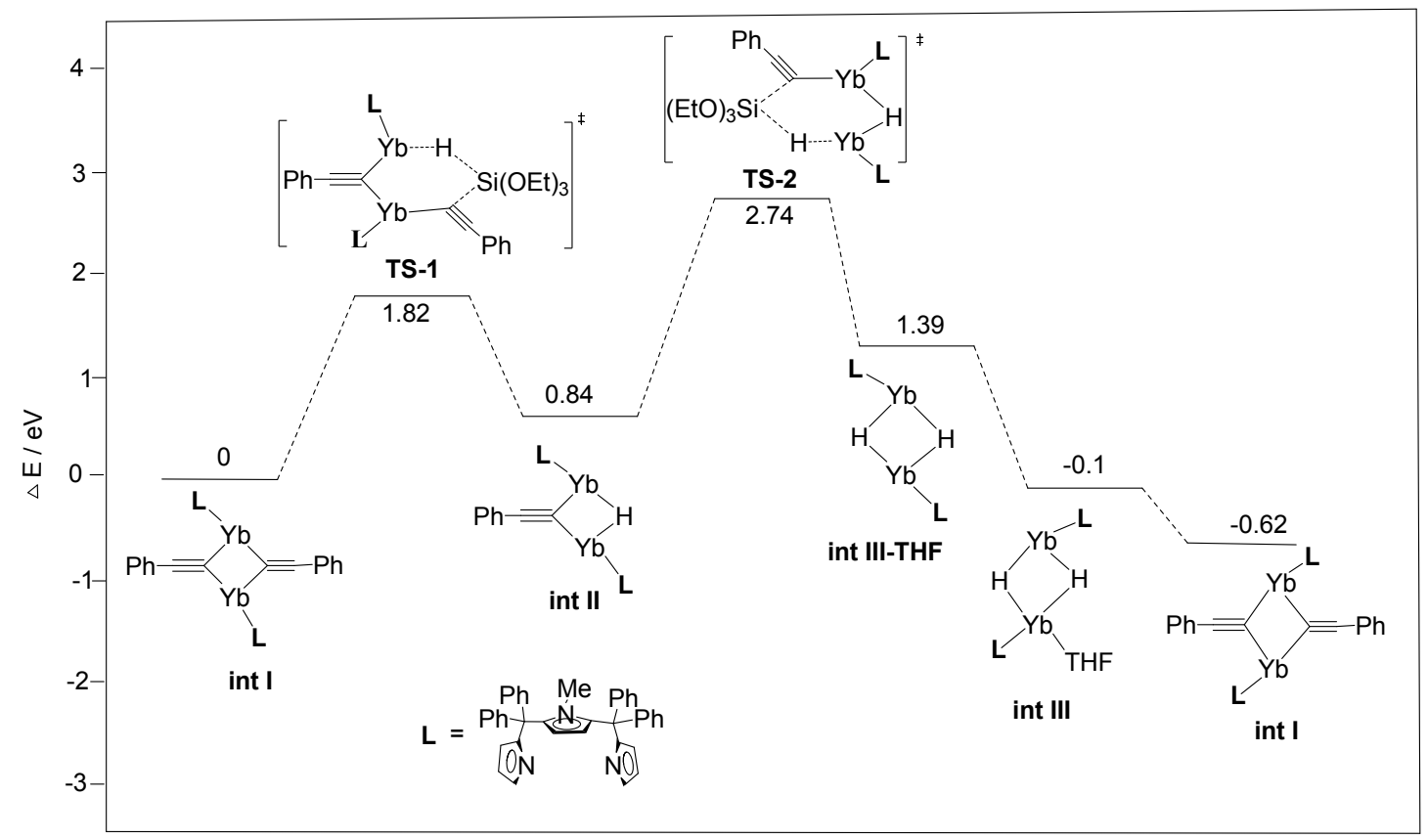

Figure S11. Energy profile of the reaction of phenylacetylene and $\mathrm{HSi}(\mathrm{OEt})_{3}$ catalyzed by $\mathbf{5 c}$ 


\section{Atomic positions}

(int I)

\begin{tabular}{|c|c|c|c|}
\hline & & & \\
\hline & 39231 & 55207 & \\
\hline & & & \\
\hline & & & \\
\hline & 40338 & & \\
\hline & 38279 & & \\
\hline & & & \\
\hline & & & \\
\hline & & & \\
\hline & & & \\
\hline & & & \\
\hline & & & \\
\hline & & & \\
\hline & & & \\
\hline & & & \\
\hline & & & \\
\hline & & & \\
\hline & & & \\
\hline & & & \\
\hline & & & \\
\hline & & & \\
\hline & & & \\
\hline & & & \\
\hline & & & \\
\hline & & & \\
\hline & & & \\
\hline & & & \\
\hline & & & \\
\hline & & & \\
\hline & & & \\
\hline & & & \\
\hline & & & \\
\hline & & & \\
\hline & & & \\
\hline & & & \\
\hline & & & \\
\hline & & & \\
\hline & & & \\
\hline & & & \\
\hline & & & \\
\hline & & & \\
\hline & & & \\
\hline & & & \\
\hline & & & \\
\hline & 0.53080 & & 0.4526 \\
\hline & 0.49366 & 0.68135 & 0.4604 \\
\hline
\end{tabular}

$\begin{array}{llll}\mathrm{C} & 0.48674 & 0.64310 & 0.46903 \\ \mathrm{C} & 0.58127 & 0.44729 & 0.57155 \\ \mathrm{C} & 0.61318 & 0.43982 & 0.59522 \\ \mathrm{C} & 0.62615 & 0.47532 & 0.60805 \\ \mathrm{C} & 0.60167 & 0.50311 & 0.59126 \\ \mathrm{C} & 0.60235 & 0.54666 & 0.59279 \\ \mathrm{C} & 0.62303 & 0.56283 & 0.55677 \\ \mathrm{C} & 0.62513 & 0.54195 & 0.52270 \\ \mathrm{C} & 0.64202 & 0.55768 & 0.49014 \\ \mathrm{C} & 0.65703 & 0.59461 & 0.49109 \\ \mathrm{C} & 0.65496 & 0.61570 & 0.52490 \\ \mathrm{C} & 0.63816 & 0.59992 & 0.55744 \\ \mathrm{C} & 0.62309 & 0.56143 & 0.62875 \\ \mathrm{C} & 0.66190 & 0.55287 & 0.63433 \\ \mathrm{C} & 0.68127 & 0.56575 & 0.66666 \\ \mathrm{C} & 0.66243 & 0.58808 & 0.69389 \\ \mathrm{C} & 0.62426 & 0.59784 & 0.68805 \\ \mathrm{C} & 0.60488 & 0.58471 & 0.65569 \\ \mathrm{C} & 0.56112 & 0.56068 & 0.59058 \\ \mathrm{C} & 0.54522 & 0.58579 & 0.56432 \\ \mathrm{C} & 0.50551 & 0.58769 & 0.57122 \\ \mathrm{C} & 0.49698 & 0.56378 & 0.60177 \\ \mathrm{C} & 0.53660 & 0.52259 & 0.64709 \\ \mathrm{C} & 0.45754 & 0.55388 & 0.61781 \\ \mathrm{C} & 0.42799 & 0.56717 & 0.58703 \\ \mathrm{C} & 0.41384 & 0.54230 & 0.55900 \\ \mathrm{C} & 0.38959 & 0.55573 & 0.53028 \\ \mathrm{C} & 0.37903 & 0.59412 & 0.52900 \\ \mathrm{C} & 0.39269 & 0.61905 & 0.55701 \\ \mathrm{C} & 0.51173 & 0.31061 & 0.53656 \\ \mathrm{C} & 0.41686 & 0.60566 & 0.58576 \\ \mathrm{C} & 0.44894 & 0.57471 & 0.65551 \\ \mathrm{C} & 0.47577 & 0.59822 & 0.67348 \\ \mathrm{C} & 0.46689 & 0.61716 & 0.70753 \\ \mathrm{C} & 0.43086 & 0.61297 & 0.72400 \\ \mathrm{C} & 0.40360 & 0.58996 & 0.70597 \\ \mathrm{C} & 0.41255 & 0.57117 & 0.67202 \\ \mathrm{C} & 0.45586 & 0.51088 & 0.62313 \\ \mathrm{C} & 0.44185 & 0.48837 & 0.65391 \\ \mathrm{C} & 0.44933 & 0.45045 & 0.64412 \\ \mathrm{C} & 0.46756 & 0.45141 & 0.60791 \\ \mathrm{C} & 0.50093 & 0.44926 & 0.51372 \\ \mathrm{C} & 0.49501 & 0.41488 & 0.52002 \\ \mathrm{C} & 0.37551 & 0.52782 \\ \mathrm{C} & 0.36228 & 0.53545 \\ \mathrm{C} & 0.32385 & 0.54354 \\ \mathrm{C} & 0.29788 & 0.54420 \\ \mathrm{C} & & \end{array}$

$\begin{array}{llll}\text { C } & 0.48674 & 0.64310 & 0.46903\end{array}$

$\begin{array}{llll}\text { C } & 0.58127 & 0.44729 & 0.57155\end{array}$

$\begin{array}{llll}\text { C } & 0.61318 & 0.43982 & 0.59522\end{array}$

$\begin{array}{llll}\text { C } & 0.62615 & 0.47532 & 0.60805\end{array}$

$\begin{array}{llll}0.60167 & 0.50311 & 0.59126\end{array}$

$\begin{array}{lll}0.60235 & 0.54666 & 0.59279\end{array}$

$\begin{array}{lll}0.62303 & 0.56283 & 0.55677\end{array}$

$\begin{array}{llll}\mathrm{C} & 0.62513 & 0.54195 & 0.52270\end{array}$

$\begin{array}{llll}\mathrm{C} & 0.64202 & 0.55768 & 0.49014\end{array}$

$\begin{array}{llll}\mathrm{C} & 0.65703 & 0.59461 & 0.49109\end{array}$

$\begin{array}{llll}\mathrm{C} & 0.65496 & 0.61570 & 0.52490\end{array}$

$\begin{array}{llll}\text { C } & 0.63816 & 0.59992 & 0.55744\end{array}$

$\begin{array}{llll}\text { C } & 0.62309 & 0.56143 & 0.62875\end{array}$

$\begin{array}{llll}\text { C } & 0.66190 & 0.55287 & 0.63433\end{array}$

$\begin{array}{llll}\mathrm{C} & 0.68127 & 0.56575 & 0.66666\end{array}$

$\begin{array}{llll}\text { C } & 0.66243 & 0.58808 & 0.69389\end{array}$

$\begin{array}{lllll}\text { C } & 0.62426 & 0.59784 & 0.68805\end{array}$

$\begin{array}{llll}\mathrm{C} & 0.60488 & 0.58471 & 0.65569\end{array}$

$\begin{array}{llll}\mathrm{C} & 0.56112 & 0.56068 & 0.59058\end{array}$

$\begin{array}{llll}\text { C } & 0.54522 & 0.58579 & 0.56432\end{array}$

$\begin{array}{llll}\mathrm{C} & 0.50551 & 0.58769 & 0.57122\end{array}$

$\begin{array}{llll}\text { C } & 0.49698 & 0.56378 & 0.60177\end{array}$

$\begin{array}{llll}\mathrm{C} & 0.53660 & 0.52259 & 0.64709\end{array}$

$\begin{array}{llll}\mathrm{C} & 0.45754 & 0.55388 & 0.61781\end{array}$

$\begin{array}{llll}\mathrm{C} & 0.42799 & 0.56717 & 0.58703\end{array}$

$\begin{array}{llll}\mathrm{C} & 0.41384 & 0.54230 & 0.55900\end{array}$

$\begin{array}{llll}\text { C } & 0.38959 & 0.55573 & 0.53028\end{array}$

$\begin{array}{llll}\mathrm{C} & 0.37903 & 0.59412 & 0.52900\end{array}$

$\begin{array}{llll}\text { C } & 0.39269 & 0.61905 & 0.55701\end{array}$

$\begin{array}{llll}\mathrm{C} & 0.41686 & 0.60566 & 0.58576\end{array}$

$\begin{array}{llll}\mathrm{C} & 0.44894 & 0.57471 & 0.65551\end{array}$

$\begin{array}{llll}\text { C } & 0.47577 & 0.59822 & 0.67348\end{array}$

$\begin{array}{llll}\mathrm{C} & 0.46689 & 0.61716 & 0.70753\end{array}$

$\begin{array}{llll}\mathrm{C} & 0.43086 & 0.61297 & 0.72400\end{array}$

$\begin{array}{llll}\mathrm{C} & 0.40360 & 0.58996 & 0.70597\end{array}$

$\begin{array}{llll}\mathrm{C} & 0.41255 & 0.57117 & 0.67202\end{array}$

$\begin{array}{llll}\text { C } & 0.45586 & 0.51088 & 0.62313\end{array}$

$\begin{array}{llll}\mathrm{C} & 0.44185 & 0.48837 & 0.65391\end{array}$

$\begin{array}{llll}\mathrm{C} & 0.44933 & 0.45045 & 0.64412\end{array}$

$\begin{array}{lllll}\mathrm{C} & 0.46756 & 0.45141 & 0.60791\end{array}$

$\begin{array}{llll}\mathrm{C} & 0.50093 & 0.44926 & 0.51372\end{array}$

$\begin{array}{llll}\mathrm{C} & 0.49501 & 0.41488 & 0.52002\end{array}$

$\begin{array}{llll}\text { C } & 0.48817 & 0.37551 & 0.52782\end{array}$

$\begin{array}{llll}\text { C } & 0.45079 & 0.36228 & 0.53545\end{array}$

$\begin{array}{llll}\text { C } & 0.44426 & 0.32385 & 0.54354\end{array}$

C $\quad 0.51173 \quad 0.310610 .53656$ 


\begin{tabular}{|c|c|c|c|}
\hline & .51857 & & \\
\hline & 0.44186 & 0.56485 & \\
\hline & 38046 & & \\
\hline & & & \\
\hline & 39192 & 0.47890 & \\
\hline & & & \\
\hline & & & \\
\hline & .34032 & & \\
\hline & .36992 & & \\
\hline & & & \\
\hline & & & \\
\hline & & & \\
\hline & & & \\
\hline & & & \\
\hline & & & \\
\hline & & & \\
\hline & & & \\
\hline & & & \\
\hline & & & \\
\hline & & & \\
\hline & & & \\
\hline & & & \\
\hline & & & \\
\hline & & & \\
\hline & & & \\
\hline & & & \\
\hline & & & \\
\hline & & & \\
\hline & & & \\
\hline & & & \\
\hline & & & \\
\hline & & & \\
\hline & & & \\
\hline & & & \\
\hline & & & \\
\hline & & & \\
\hline & & & \\
\hline & & & \\
\hline & & & \\
\hline & 0.64 & & \\
\hline & & & \\
\hline & & & \\
\hline & & & \\
\hline $\mathrm{H}$ & & & \\
\hline & 0.63674 & & 0.5837 \\
\hline & & & \\
\hline & & & \\
\hline $\mathrm{H}$ & 0.67757 & 0.59813 & \\
\hline & 0.60929 & 0.61588 & 0.7086 \\
\hline
\end{tabular}

$\begin{array}{llll}\mathrm{H} & 0.57523 & 0.59324 & 0.65121 \\ \mathrm{H} & 0.56060 & 0.60021 & 0.54166 \\ \mathrm{H} & 0.48487 & 0.60367 & 0.55460 \\ \mathrm{H} & 0.53157 & 0.53891 & 0.67342 \\ \mathrm{H} & 0.51705 & 0.49822 & 0.64610 \\ \mathrm{H} & 0.56595 & 0.51163 & 0.64710 \\ \mathrm{H} & 0.42205 & 0.51223 & 0.55937 \\ \mathrm{H} & 0.37904 & 0.53599 & 0.50854 \\ \mathrm{H} & 0.36020 & 0.60450 & 0.50640 \\ \mathrm{H} & 0.38463 & 0.64919 & 0.55656 \\ \mathrm{H} & 0.42745 & 0.62544 & 0.60743 \\ \mathrm{H} & 0.50375 & 0.60248 & 0.66038 \\ \mathrm{H} & 0.48826 & 0.63547 & 0.72097 \\ \mathrm{H} & 0.42385 & 0.62779 & 0.75054 \\ \mathrm{H} & 0.37510 & 0.58685 & 0.71828 \\ \mathrm{H} & 0.39087 & 0.55390 & 0.65767 \\ \mathrm{H} & 0.42878 & 0.49885 & 0.68000 \\ \mathrm{H} & 0.44276 & 0.42506 & 0.66073 \\ \mathrm{H} & 0.47759 & 0.42765 & 0.59060 \\ \mathrm{H} & 0.42717 & 0.38265 & 0.53497 \\ \mathrm{H} & 0.41520 & 0.31410 & 0.54941 \\ \mathrm{H} & 0.46942 & 0.26780 & 0.55063 \\ \mathrm{H} & 0.53556 & 0.29047 & 0.53706 \\ \mathrm{H} & 0.54749 & 0.35900 & 0.52221 \\ \mathrm{~N} & 0.43109 & 0.50655 & 0.42865 \\ \mathrm{~N} & 0.47425 & 0.44501 & 0.38388 \\ \mathrm{~N} & 0.53398 & 0.50431 & 0.40214 \\ \mathrm{~N} & 0.57429 & 0.48556 & 0.56919 \\ \mathrm{~N} & 0.53142 & 0.54713 & 0.61365 \\ \mathrm{~N} & 0.47159 & 0.48796 & 0.59528 \\ \mathrm{Yb} & 0.49041 & 0.48126 & 0.45065 \\ \mathrm{Yb} & 0.51519 & 0.51090 & 0.54685\end{array}$

\section{TS1:}

$\begin{array}{llll}\mathrm{C} & 0.36522 & 0.53123 & 0.42185 \\ \mathrm{C} & 0.33363 & 0.53851 & 0.39802 \\ \mathrm{C} & 0.32907 & 0.50578 & 0.37548 \\ \mathrm{C} & 0.35785 & 0.47962 & 0.38734 \\ \mathrm{C} & 0.36088 & 0.43656 & 0.38131 \\ \mathrm{C} & 0.34029 & 0.41623 & 0.41571 \\ \mathrm{C} & 0.33515 & 0.43432 & 0.45106 \\ \mathrm{C} & 0.31727 & 0.41549 & 0.48138 \\ \mathrm{C} & 0.30435 & 0.37803 & 0.47710 \\ \mathrm{C} & 0.30954 & 0.35958 & 0.44209 \\ \mathrm{C} & 0.32720 & 0.37852 & 0.41172 \\ \mathrm{C} & 0.34046 & 0.42342 & 0.34453 \\ \mathrm{C} & 0.30107 & 0.43008 & 0.34035 \\ \mathrm{C} & 0.28150 & 0.41810 & 0.30781 \\ \mathrm{C} & 0.30068 & 0.39828 & 0.27891\end{array}$




\begin{tabular}{|c|c|c|c|}
\hline $\mathrm{C}$ & 0.33945 & 0.39021 & 0.28332 \\
\hline $\mathrm{C}$ & 0.35904 & 0.40262 & 0.31589 \\
\hline $\mathrm{C}$ & 0.40282 & 0.42472 & 0.38313 \\
\hline $\mathrm{C}$ & 0.41904 & 0.39945 & 0.40884 \\
\hline $\mathrm{C}$ & 0.45879 & 0.39844 & 0.40242 \\
\hline $\mathrm{C}$ & 0.46741 & 0.42282 & 0.37235 \\
\hline $\mathrm{C}$ & 0.42755 & 0.46369 & 0.32700 \\
\hline $\mathrm{C}$ & 0.50694 & 0.42980 & 0.35498 \\
\hline $\mathrm{C}$ & 0.53626 & 0.41475 & 0.38531 \\
\hline $\mathrm{C}$ & 0.55250 & 0.43872 & 0.41295 \\
\hline $\mathrm{C}$ & 0.57681 & 0.42404 & 0.44103 \\
\hline $\mathrm{C}$ & 0.58519 & 0.38508 & 0.44211 \\
\hline $\mathrm{C}$ & 0.56922 & 0.36092 & 0.41462 \\
\hline $\mathrm{C}$ & 0.54515 & 0.37567 & 0.38643 \\
\hline $\mathrm{C}$ & 0.51373 & 0.40803 & 0.31737 \\
\hline $\mathrm{C}$ & 0.48517 & 0.38731 & 0.29870 \\
\hline $\mathrm{C}$ & 0.49269 & 0.36853 & 0.26419 \\
\hline $\mathrm{C}$ & 0.52902 & 0.37014 & 0.24792 \\
\hline $\mathrm{C}$ & 0.55806 & 0.39005 & 0.26681 \\
\hline $\mathrm{C}$ & 0.55051 & 0.40844 & 0.30127 \\
\hline $\mathrm{C}$ & 0.51318 & 0.47221 & 0.34862 \\
\hline $\mathrm{C}$ & 0.53377 & 0.49202 & 0.31951 \\
\hline $\mathrm{C}$ & 0.53418 & 0.53044 & 0.33038 \\
\hline $\mathrm{C}$ & 0.51337 & 0.53250 & 0.36478 \\
\hline $\mathrm{C}$ & 0.45462 & 0.58723 & 0.48135 \\
\hline $\mathrm{C}$ & 0.47073 & 0.61473 & 0.49723 \\
\hline $\mathrm{C}$ & 0.48712 & 0.64900 & 0.51122 \\
\hline $\mathrm{C}$ & 0.52671 & 0.65601 & 0.50794 \\
\hline $\mathrm{C}$ & 0.54205 & 0.69025 & 0.52105 \\
\hline $\mathrm{C}$ & 0.51834 & 0.71783 & 0.53760 \\
\hline $\mathrm{C}$ & 0.47906 & 0.71120 & 0.54085 \\
\hline $\mathrm{C}$ & 0.46341 & 0.67711 & 0.52771 \\
\hline $\mathrm{C}$ & 0.54184 & 0.45346 & 0.53787 \\
\hline $\mathrm{C}$ & 0.57516 & 0.44040 & 0.55633 \\
\hline $\mathrm{C}$ & 0.58809 & 0.47078 & 0.57899 \\
\hline $\mathrm{C}$ & 0.56214 & 0.50116 & 0.57305 \\
\hline $\mathrm{C}$ & 0.56393 & 0.54253 & 0.58624 \\
\hline $\mathrm{C}$ & 0.58480 & 0.56669 & 0.55484 \\
\hline $\mathrm{C}$ & 0.58543 & 0.55477 & 0.51658 \\
\hline $\mathrm{C}$ & 0.60403 & 0.57636 & 0.48863 \\
\hline $\mathrm{C}$ & 0.62250 & 0.61037 & 0.49835 \\
\hline $\mathrm{C}$ & 0.62177 & 0.62272 & 0.53630 \\
\hline $\mathrm{C}$ & 0.60301 & 0.60118 & 0.56420 \\
\hline $\mathrm{C}$ & 0.58577 & 0.54772 & 0.62415 \\
\hline $\mathrm{C}$ & 0.62506 & 0.53968 & 0.62610 \\
\hline $\mathrm{C}$ & 0.64577 & 0.54662 & 0.65942 \\
\hline $\mathrm{C}$ & 0.62777 & 0.56208 & 0.69156 \\
\hline $\mathrm{C}$ & 0.58890 & 0.57059 & 0.68985 \\
\hline $\mathrm{C}$ & 0.56824 & 0.56357 & 0.65640 \\
\hline
\end{tabular}

\begin{tabular}{|c|c|c|c|}
\hline C & 0.52333 & 0.55778 & 0.58912 \\
\hline $\mathrm{C}$ & 0.51005 & 0.59174 & 0.57409 \\
\hline $\mathrm{C}$ & 0.47034 & 0.59385 & 0.58078 \\
\hline $\mathrm{C}$ & 0.45929 & 0.56127 & 0.60024 \\
\hline $\mathrm{C}$ & 0.49575 & 0.50600 & 0.63127 \\
\hline $\mathrm{C}$ & 0.41887 & 0.55136 & 0.61300 \\
\hline $\mathrm{C}$ & 0.39081 & 0.57414 & 0.58656 \\
\hline $\mathrm{C}$ & 0.37386 & 0.55725 & 0.55442 \\
\hline $\mathrm{C}$ & 0.34930 & 0.57804 & 0.53081 \\
\hline $\mathrm{C}$ & 0.34115 & 0.61621 & 0.53886 \\
\hline $\mathrm{C}$ & 0.35789 & 0.63340 & 0.57074 \\
\hline $\mathrm{C}$ & 0.38234 & 0.61256 & 0.59441 \\
\hline $\mathrm{C}$ & 0.41172 & 0.56394 & 0.65456 \\
\hline $\mathrm{C}$ & 0.44069 & 0.57967 & 0.67715 \\
\hline $\mathrm{C}$ & 0.43303 & 0.59325 & 0.71395 \\
\hline $\mathrm{C}$ & 0.39607 & 0.59140 & 0.72871 \\
\hline $\mathrm{C}$ & 0.36685 & 0.57577 & 0.70639 \\
\hline $\mathrm{C}$ & 0.37460 & 0.56230 & 0.66972 \\
\hline $\mathrm{C}$ & 0.41271 & 0.50897 & 0.60727 \\
\hline $\mathrm{C}$ & 0.39238 & 0.48221 & 0.62961 \\
\hline $\mathrm{C}$ & 0.39477 & 0.44741 & 0.61002 \\
\hline $\mathrm{C}$ & 0.41656 & 0.45457 & 0.57680 \\
\hline $\mathrm{C}$ & 0.45744 & 0.46496 & 0.48791 \\
\hline $\mathrm{C}$ & 0.45525 & 0.42969 & 0.49181 \\
\hline $\mathrm{C}$ & 0.45169 & 0.38953 & 0.49892 \\
\hline $\mathrm{C}$ & 0.41539 & 0.37313 & 0.50573 \\
\hline $\mathrm{C}$ & 0.41171 & 0.33413 & 0.51290 \\
\hline $\mathrm{C}$ & 0.44397 & 0.31057 & 0.51369 \\
\hline $\mathrm{C}$ & 0.48004 & 0.32637 & 0.50702 \\
\hline $\mathrm{C}$ & 0.48399 & 0.36532 & 0.49958 \\
\hline $\mathrm{C}$ & 0.40079 & 0.68133 & 0.44048 \\
\hline $\mathrm{C}$ & 0.38914 & 0.64104 & 0.45168 \\
\hline $\mathrm{C}$ & 0.52720 & 0.66899 & 0.40714 \\
\hline $\mathrm{C}$ & 0.51767 & 0.62679 & 0.41052 \\
\hline $\mathrm{C}$ & 0.43802 & 0.60210 & 0.34146 \\
\hline $\mathrm{C}$ & 0.42411 & 0.56527 & 0.35989 \\
\hline $\mathrm{H}$ & 0.37756 & 0.55018 & 0.44313 \\
\hline $\mathrm{H}$ & 0.31697 & 0.56472 & 0.39694 \\
\hline $\mathrm{H}$ & 0.30776 & 0.50137 & 0.35342 \\
\hline $\mathrm{H}$ & 0.34570 & 0.46326 & 0.45527 \\
\hline $\mathrm{H}$ & 0.31368 & 0.43043 & 0.50856 \\
\hline $\mathrm{H}$ & 0.29029 & 0.36339 & 0.50080 \\
\hline $\mathrm{H}$ & 0.29957 & 0.33026 & 0.43821 \\
\hline $\mathrm{H}$ & 0.33076 & 0.36369 & 0.38453 \\
\hline $\mathrm{H}$ & 0.28541 & 0.44410 & 0.36335 \\
\hline $\mathrm{H}$ & 0.25094 & 0.42388 & 0.30524 \\
\hline $\mathrm{H}$ & 0.28532 & 0.38874 & 0.25349 \\
\hline $\mathrm{H}$ & 0.35466 & 0.37392 & 0.26149 \\
\hline $\mathrm{H}$ & 0.38909 & 0.39510 & 0.31934 \\
\hline
\end{tabular}




\begin{tabular}{|c|c|c|c|}
\hline & .40370 & & \\
\hline & 0.47940 & 0.38210 & \\
\hline & .42771 & 0.44647 & 0.30074 \\
\hline & 0.45079 & 0.48462 & \\
\hline & 0.40007 & 0.47865 & \\
\hline & .54582 & 0.46914 & \\
\hline & .58889 & & \\
\hline & .60410 & & \\
\hline & .57543 & 0.33034 & \\
\hline & & & \\
\hline & & & \\
\hline & 47001 & & \\
\hline & 53492 & & \\
\hline & .58682 & & \\
\hline & & & \\
\hline & 0.54579 & & \\
\hline & .54732 & & \\
\hline & & & \\
\hline & & & \\
\hline & .57276 & & \\
\hline & 0.53050 & & \\
\hline & & & \\
\hline & & & \\
\hline & 0.52339 & & \\
\hline & 0.58797 & & \\
\hline & 92 & & \\
\hline & & & \\
\hline & 60421 & & \\
\hline & & & \\
\hline & 605 & & \\
\hline & & & \\
\hline & 0.63982 & & \\
\hline & 0.67 & & \\
\hline & 0.6 & & \\
\hline & & & \\
\hline & 0.53817 & & \\
\hline & & & \\
\hline & 0.45108 & & \\
\hline & 0.49708 & 0.5 & \\
\hline & & & \\
\hline & 0.52207 & 0.4 & 0.6 \\
\hline & 0.37972 & & \\
\hline & 0.33647 & & 0.5 \\
\hline & & & \\
\hline & 0.35166 & & \\
\hline & 0.39477 & 0.62630 & \\
\hline & 0.46952 & & \\
\hline & & & \\
\hline & 0.38992 & 0.60239 & $0.75^{\prime}$ \\
\hline
\end{tabular}

$\begin{array}{cccc}\mathrm{H} & 0.33768 & 0.57456 & 0.71741 \\ \mathrm{H} & 0.35139 & 0.55124 & 0.65207 \\ \mathrm{H} & 0.37830 & 0.48767 & 0.65671 \\ \mathrm{H} & 0.38251 & 0.42014 & 0.61848 \\ \mathrm{H} & 0.42460 & 0.43467 & 0.55444 \\ \mathrm{H} & 0.39007 & 0.39129 & 0.50485 \\ \mathrm{H} & 0.38334 & 0.32205 & 0.51774 \\ \mathrm{H} & 0.44100 & 0.28003 & 0.51945 \\ \mathrm{H} & 0.50537 & 0.30815 & 0.50754 \\ \mathrm{H} & 0.51221 & 0.37730 & 0.49391 \\ \mathrm{H} & 0.48780 & 0.54242 & 0.44612 \\ \mathrm{H} & 0.39009 & 0.68841 & 0.41180 \\ \mathrm{H} & 0.43207 & 0.68414 & 0.44029 \\ \mathrm{H} & 0.38900 & 0.70212 & 0.46093 \\ \mathrm{H} & 0.39798 & 0.63468 & 0.48128 \\ \mathrm{H} & 0.35779 & 0.63783 & 0.45072 \\ \mathrm{H} & 0.51717 & 0.68476 & 0.43238 \\ \mathrm{H} & 0.51321 & 0.68112 & 0.38175 \\ \mathrm{H} & 0.55824 & 0.67322 & 0.40443 \\ \mathrm{H} & 0.52847 & 0.61135 & 0.38519 \\ \mathrm{H} & 0.53130 & 0.61369 & 0.43580 \\ \mathrm{H} & 0.46922 & 0.60261 & 0.33804 \\ \mathrm{H} & 0.42928 & 0.62751 & 0.35760 \\ \mathrm{H} & 0.42511 & 0.60391 & 0.31280 \\ \mathrm{H} & 0.39284 & 0.56549 & 0.36269 \\ \mathrm{H} & 0.43258 & 0.54092 & 0.34206 \\ \mathrm{~N} & 0.38078 & 0.49615 & 0.41470 \\ \mathrm{~N} & 0.43276 & 0.43987 & 0.36086 \\ \mathrm{~N} & 0.50010 & 0.49726 & 0.37549 \\ \mathrm{~N} & 0.53362 & 0.49032 & 0.54795 \\ \mathrm{~N} & 0.49221 & 0.53895 & 0.60576 \\ \mathrm{~N} & 0.42767 & 0.49191 & 0.57490 \\ \mathrm{Yb} & 0.44942 & 0.49021 & 0.42126 \\ \mathrm{Yb} & 0.47416 & 0.51988 & 0.53130 \\ \mathrm{O} & 0.47732 & 0.62297 & 0.41258 \\ \mathrm{O} & 0.44016 & 0.55638 & 0.39788 \\ \mathrm{O} & 0.40444 & 0.61300 & 0.42638 \\ \mathrm{Si} & 0.44671 & 0.59166 & 0.42949\end{array}$

\section{Int II}

$\begin{array}{llll}\mathrm{C} & 0.38294 & 0.56827 & 0.39669 \\ \mathrm{C} & 0.35157 & 0.57658 & 0.37254 \\ \mathrm{C} & 0.33984 & 0.54170 & 0.35705 \\ \mathrm{C} & 0.36442 & 0.51343 & 0.37269 \\ \mathrm{C} & 0.36476 & 0.47003 & 0.36858 \\ \mathrm{C} & 0.34394 & 0.45088 & 0.40304 \\ \mathrm{C} & 0.33972 & 0.46950 & 0.43819 \\ \mathrm{C} & 0.32331 & 0.45068 & 0.46936 \\ \mathrm{C} & 0.31079 & 0.41296 & 0.46590\end{array}$ 


\begin{tabular}{|c|c|c|c|}
\hline & 31478 & & \\
\hline & 33116 & 0.41301 & \\
\hline & 34488 & 0.45672 & \\
\hline & 30583 & 0.46427 & \\
\hline & 28680 & 0.45180 & \\
\hline & 30627 & 0.43090 & \\
\hline & 34475 & 0.42224 & \\
\hline & & & \\
\hline & 40629 & 0.45694 & \\
\hline & & & \\
\hline & & & \\
\hline & & & \\
\hline & & 0.4 & \\
\hline & 8 & & \\
\hline & & & \\
\hline & & & \\
\hline & 76 & & \\
\hline & & 0.4 & \\
\hline & & & \\
\hline & & 0.4 & \\
\hline & 624 & 0.4 & \\
\hline & & 0.4 & \\
\hline & & & \\
\hline & & & \\
\hline & 35 & 0.4 & \\
\hline & 22 & 0.4 & \\
\hline & & & \\
\hline & & & \\
\hline & 5 & & \\
\hline & & & \\
\hline & & & \\
\hline & 27 & & \\
\hline & 1 & & \\
\hline & 0.56 & 0.5 & \\
\hline & 0.56 & & \\
\hline & 0.58718 & & \\
\hline & 0.5 & 0.5 & \\
\hline & 0.60904 & 0.5 & \\
\hline & 0.62379 & 0.63486 & \\
\hline & & & \\
\hline 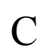 & 0.60 & 0.6 & \\
\hline $\mathrm{C}$ & 0.58502 & 0.57828 & \\
\hline $\mathrm{C}$ & 0.62406 & 0.56969 & \\
\hline $\mathrm{C}$ & & & \\
\hline $\mathrm{C}$ & 0.62363 & 0.59146 & \\
\hline $\mathrm{C}$ & 0.58531 & 0.60161 & \\
\hline $\mathrm{C}$ & 0.56635 & & \\
\hline $\mathrm{C}$ & & & \\
\hline $\mathrm{C}$ & 0.50841 & 0.61064 & 0.53 \\
\hline
\end{tabular}

\begin{tabular}{|c|c|c|c|}
\hline $\mathrm{C}$ & 0.46870 & 0.61290 & 0.54279 \\
\hline $\mathrm{C}$ & 0.45979 & 0.58723 & 0.57181 \\
\hline $\mathrm{C}$ & 0.49755 & 0.53866 & 0.61085 \\
\hline $\mathrm{C}$ & 0.42052 & 0.57869 & 0.58877 \\
\hline $\mathrm{C}$ & 0.39108 & 0.60043 & 0.56324 \\
\hline $\mathrm{C}$ & 0.37455 & 0.58336 & 0.53102 \\
\hline $\mathrm{C}$ & 0.35024 & 0.60417 & 0.50713 \\
\hline $\mathrm{C}$ & 0.34190 & 0.64233 & 0.51514 \\
\hline $\mathrm{C}$ & 0.35813 & 0.65956 & 0.54729 \\
\hline $\mathrm{C}$ & 0.38255 & 0.63881 & 0.57106 \\
\hline $\mathrm{C}$ & 0.41613 & 0.59270 & 0.63031 \\
\hline $\mathrm{C}$ & 0.44604 & 0.60925 & 0.65101 \\
\hline $\mathrm{C}$ & 0.44085 & 0.62103 & 0.68887 \\
\hline $\mathrm{C}$ & 0.40539 & 0.61683 & 0.70653 \\
\hline $\mathrm{C}$ & 0.37494 & 0.60141 & 0.68576 \\
\hline $\mathrm{C}$ & 0.38026 & 0.58974 & 0.64807 \\
\hline $\mathrm{C}$ & 0.41408 & 0.53579 & 0.58656 \\
\hline $\mathrm{C}$ & 0.39562 & 0.51042 & 0.61241 \\
\hline $\mathrm{C}$ & 0.39766 & 0.47425 & 0.59581 \\
\hline $\mathrm{C}$ & 0.41754 & 0.47892 & 0.56087 \\
\hline $\mathrm{C}$ & 0.46555 & 0.47509 & 0.47823 \\
\hline $\mathrm{C}$ & 0.46098 & 0.44022 & 0.48247 \\
\hline $\mathrm{C}$ & 0.45606 & 0.40057 & 0.48994 \\
\hline $\mathrm{C}$ & 0.41939 & 0.38551 & 0.49743 \\
\hline $\mathrm{C}$ & 0.41497 & 0.34706 & 0.50673 \\
\hline $\mathrm{C}$ & 0.44679 & 0.32296 & 0.50862 \\
\hline $\mathrm{C}$ & 0.48319 & 0.33747 & 0.50073 \\
\hline $\mathrm{C}$ & 0.48791 & 0.37586 & 0.49135 \\
\hline $\mathrm{H}$ & 0.39996 & 0.58764 & 0.41422 \\
\hline $\mathrm{H}$ & 0.33920 & 0.60458 & 0.36724 \\
\hline $\mathrm{H}$ & 0.31676 & 0.53718 & 0.33687 \\
\hline $\mathrm{H}$ & 0.34977 & 0.49882 & 0.44143 \\
\hline $\mathrm{H}$ & 0.32055 & 0.46569 & 0.49658 \\
\hline $\mathrm{H}$ & 0.29800 & 0.39824 & 0.49025 \\
\hline $\mathrm{H}$ & 0.30520 & 0.36467 & 0.42768 \\
\hline $\mathrm{H}$ & 0.33427 & 0.39793 & 0.37268 \\
\hline $\mathrm{H}$ & 0.28995 & 0.47939 & 0.34840 \\
\hline $\mathrm{H}$ & 0.25643 & 0.45806 & 0.29002 \\
\hline $\mathrm{H}$ & 0.29137 & 0.42103 & 0.24002 \\
\hline $\mathrm{H}$ & 0.36022 & 0.40524 & 0.24998 \\
\hline $\mathrm{H}$ & 0.39350 & 0.42683 & 0.30819 \\
\hline $\mathrm{H}$ & 0.40668 & 0.41636 & 0.41908 \\
\hline $\mathrm{H}$ & 0.48250 & 0.41352 & 0.40665 \\
\hline $\mathrm{H}$ & 0.42841 & 0.48084 & 0.28945 \\
\hline $\mathrm{H}$ & 0.45627 & 0.51602 & 0.31388 \\
\hline $\mathrm{H}$ & 0.40573 & 0.51453 & 0.32066 \\
\hline $\mathrm{H}$ & 0.54913 & 0.49988 & 0.40330 \\
\hline $\mathrm{H}$ & 0.59164 & 0.47125 & 0.45182 \\
\hline $\mathrm{H}$ & 0.60655 & 0.40161 & 0.44993 \\
\hline
\end{tabular}




\begin{tabular}{|c|c|c|c|}
\hline $\mathrm{H}$ & 0.57857 & 0.36113 & 0.39815 \\
\hline$\theta$ & 0.53623 & 0.38990 & 0.34952 \\
\hline H & 0.46020 & 0.41882 & 0.30120 \\
\hline $\mathrm{H}$ & 0.47241 & 0.38596 & 0.24000 \\
\hline H & 0.53639 & 0.39074 & 0.20886 \\
\hline H & 0.58766 & 0.42934 & 0.23996 \\
\hline $\mathrm{H}$ & 0.57519 & 0.46232 & 0.30122 \\
\hline H & 0.54476 & 0.52220 & 0.28809 \\
\hline $\mathrm{H}$ & 0.53536 & 0.59461 & 0.31401 \\
\hline $\mathrm{H}$ & 0.49611 & 0.58807 & 0.38172 \\
\hline $\mathrm{H}$ & 0.52884 & 0.45611 & 0.50828 \\
\hline H & 0.59063 & 0.43572 & 0.55217 \\
\hline $\mathrm{H}$ & 0.61350 & 0.50054 & 0.58742 \\
\hline $\mathrm{H}$ & 0.57912 & 0.54935 & 0.48658 \\
\hline $\mathrm{H}$ & 0.61145 & 0.58690 & 0.43608 \\
\hline $\mathrm{H}$ & 0.63800 & 0.65147 & 0.45075 \\
\hline $\mathrm{H}$ & 0.63154 & 0.67786 & 0.51672 \\
\hline $\mathrm{H}$ & 0.59928 & 0.64026 & 0.56700 \\
\hline $\mathrm{H}$ & 0.63980 & 0.55846 & 0.58027 \\
\hline $\mathrm{H}$ & 0.67331 & 0.56855 & 0.64153 \\
\hline $\mathrm{H}$ & 0.63843 & 0.59602 & 0.69743 \\
\hline $\mathrm{H}$ & 0.56992 & 0.61458 & 0.69015 \\
\hline $\mathrm{H}$ & 0.53666 & 0.60422 & 0.62889 \\
\hline $\mathrm{H}$ & 0.52439 & 0.62683 & 0.51469 \\
\hline $\mathrm{H}$ & 0.44831 & 0.63110 & 0.52823 \\
\hline $\mathrm{H}$ & 0.49809 & 0.55097 & 0.63970 \\
\hline $\mathrm{H}$ & 0.47349 & 0.51876 & 0.60867 \\
\hline $\mathrm{H}$ & 0.52419 & 0.52295 & 0.60573 \\
\hline $\mathrm{H}$ & 0.38103 & 0.55363 & 0.52430 \\
\hline $\mathrm{H}$ & 0.33802 & 0.59037 & 0.48195 \\
\hline $\mathrm{H}$ & 0.32295 & 0.65861 & 0.49645 \\
\hline $\mathrm{H}$ & 0.35194 & 0.68942 & 0.55393 \\
\hline $\mathrm{H}$ & 0.39520 & 0.65273 & 0.59594 \\
\hline $\mathrm{H}$ & 0.47372 & 0.61366 & 0.63737 \\
\hline $\mathrm{H}$ & 0.46473 & 0.63370 & 0.70444 \\
\hline $\mathrm{H}$ & 0.40131 & 0.62584 & 0.73611 \\
\hline $\mathrm{H}$ & 0.34678 & 0.59861 & 0.69891 \\
\hline $\mathrm{H}$ & 0.35608 & 0.57852 & 0.63185 \\
\hline $\mathrm{H}$ & 0.38304 & 0.51767 & 0.63980 \\
\hline $\mathrm{H}$ & 0.38657 & 0.44756 & 0.60726 \\
\hline $\mathrm{H}$ & 0.42509 & 0.45745 & 0.53978 \\
\hline $\mathrm{H}$ & 0.39462 & 0.40443 & 0.49604 \\
\hline $\mathrm{H}$ & 0.38647 & 0.33587 & 0.51268 \\
\hline $\mathrm{H}$ & 0.44326 & 0.29294 & 0.51629 \\
\hline $\mathrm{H}$ & 0.50812 & 0.31876 & 0.50212 \\
\hline $\mathrm{H}$ & 0.51624 & 0.38734 & 0.48514 \\
\hline $\mathrm{H}$ & 0.46282 & 0.55852 & 0.45332 \\
\hline $\mathrm{N}$ & 0.39084 & 0.53015 & 0.39666 \\
\hline $\mathrm{N}$ & 0.43618 & 0.47135 & 0.34867 \\
\hline
\end{tabular}

$\begin{array}{cccc}\mathrm{N} & 0.49786 & 0.52824 & 0.37112 \\ \mathrm{~N} & 0.53840 & 0.51220 & 0.52947 \\ \mathrm{~N} & 0.49375 & 0.56856 & 0.58188 \\ \mathrm{~N} & 0.42770 & 0.51607 & 0.55526 \\ \mathrm{Yb} & 0.45143 & 0.50669 & 0.41668 \\ \mathrm{Yb} & 0.47697 & 0.53765 & 0.51145\end{array}$

\section{TS2:}

$\begin{array}{llll}\text { C } & 0.38100 & 0.59168 & 0.41559\end{array}$

$\begin{array}{llll}\text { C } & 0.34937 & 0.60122 & 0.39261\end{array}$

$\begin{array}{llll}\text { C } & 0.33609 & 0.56678 & 0.37688\end{array}$

$\begin{array}{llll}\text { C } & 0.36016 & 0.53778 & 0.39117\end{array}$

$\begin{array}{llll}\text { C } & 0.35898 & 0.49455 & 0.38604\end{array}$

$\begin{array}{llll}\text { C } & 0.33827 & 0.47531 & 0.42056\end{array}$

$\begin{array}{llll}\text { C } & 0.33432 & 0.49396 & 0.45577\end{array}$

$\begin{array}{llll}\text { C } & 0.31802 & 0.47524 & 0.48707\end{array}$

$\begin{array}{llll}\text { C } & 0.30529 & 0.43758 & 0.48371\end{array}$

$\begin{array}{llll}\text { C } & 0.30875 & 0.41886 & 0.44861\end{array}$

$\begin{array}{llll}\text { C } & 0.32507 & 0.43757 & 0.41738\end{array}$

$\begin{array}{llll}\text { C } & 0.33862 & 0.48178 & 0.34916\end{array}$

$\begin{array}{llll}\text { C } & 0.29958 & 0.48958 & 0.34401\end{array}$

$\begin{array}{llll}\text { C } & 0.28022 & 0.47686 & 0.31158\end{array}$

$\begin{array}{llll}\text { C } & 0.29932 & 0.45562 & 0.28366\end{array}$

$\begin{array}{llll}\text { C } & 0.33782 & 0.44684 & 0.28887\end{array}$

$\begin{array}{llll}\text { C } & 0.35711 & 0.45965 & 0.32142\end{array}$

$\begin{array}{llll}\text { C } & 0.40022 & 0.48079 & 0.38739\end{array}$

$\begin{array}{llll}\text { C } & 0.41561 & 0.45445 & 0.41251\end{array}$

$\begin{array}{llll}\text { C } & 0.45548 & 0.45286 & 0.40626\end{array}$

$\begin{array}{llll}\text { C } & 0.46435 & 0.47825 & 0.37720\end{array}$

$\begin{array}{llll}\text { C } & 0.42566 & 0.52217 & 0.33373\end{array}$

$\begin{array}{llll}\text { C } & 0.50389 & 0.48807 & 0.36197\end{array}$

$\begin{array}{llll}\text { C } & 0.53316 & 0.47085 & 0.39093\end{array}$

$\begin{array}{llll}\text { C } & 0.54932 & 0.49311 & 0.42005\end{array}$

$\begin{array}{llll}\text { C } & 0.57318 & 0.47671 & 0.44755\end{array}$

$\begin{array}{llll}\text { C } & 0.58139 & 0.43773 & 0.44644\end{array}$

$\begin{array}{llll}\text { C } & 0.56592 & 0.41537 & 0.41719\end{array}$

$\begin{array}{llll}\text { C } & 0.54215 & 0.43179 & 0.38966\end{array}$

$\begin{array}{llll}\text { C } & 0.51057 & 0.46955 & 0.32261\end{array}$

$\begin{array}{llll}\text { C } & 0.48246 & 0.44722 & 0.30489\end{array}$

$\begin{array}{llll}\text { C } & 0.48972 & 0.42827 & 0.27050\end{array}$

$\begin{array}{llll}\text { C } & 0.52550 & 0.43121 & 0.25323\end{array}$

$\begin{array}{llll}\text { C } & 0.55389 & 0.45323 & 0.27072\end{array}$

$\begin{array}{llll}\text { C } & 0.54655 & 0.47207 & 0.30506\end{array}$

$\begin{array}{llll}\text { C } & 0.50676 & 0.53139 & 0.36160\end{array}$

$\begin{array}{llll}\text { C } & 0.52402 & 0.55659 & 0.33537\end{array}$

$\begin{array}{llll}\text { C } & 0.51758 & 0.59361 & 0.34952\end{array}$

$\begin{array}{llll}\text { C } & 0.49671 & 0.58917 & 0.38369\end{array}$

$\begin{array}{llll}\text { C } & 0.55287 & 0.50980 & 0.55007\end{array}$

$\begin{array}{llll}\text { C } & 0.57951 & 0.49891 & 0.57846\end{array}$ 


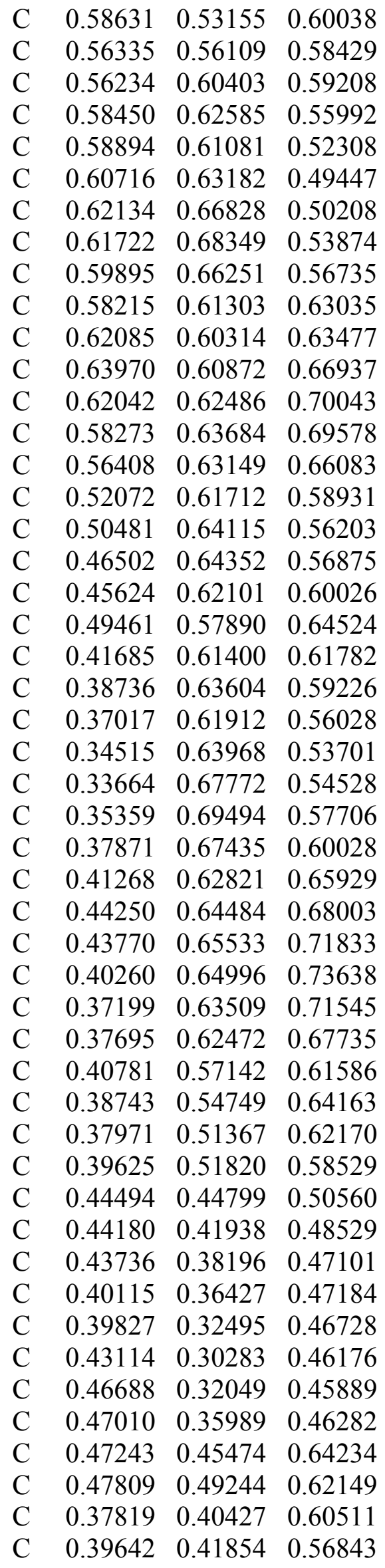

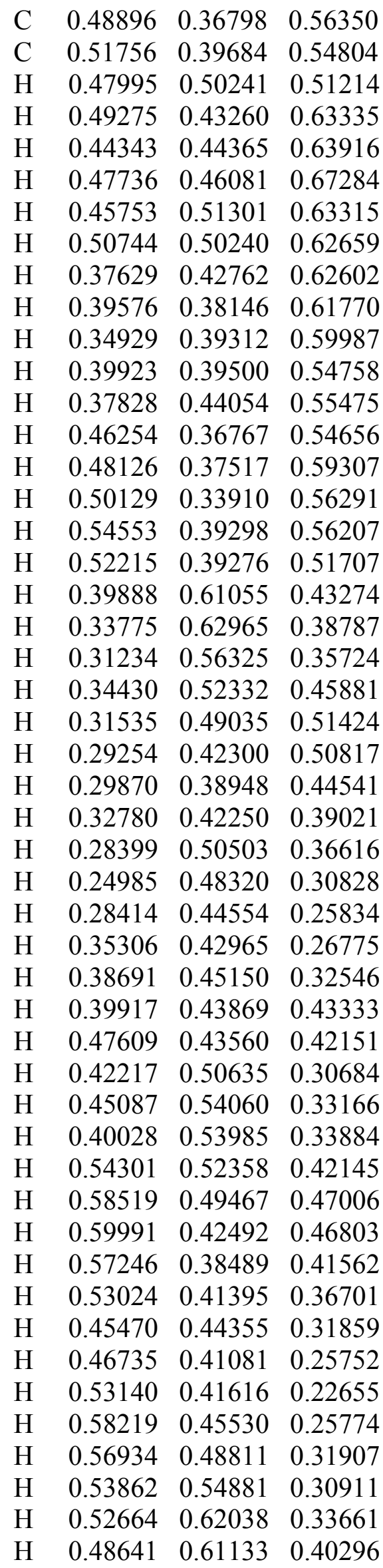




\begin{tabular}{|c|c|c|c|c|c|c|c|}
\hline $\mathrm{H}$ & 0.54102 & 0.49265 & 0.52704 & \multirow{2}{*}{\multicolumn{2}{|c|}{ Int III-THF }} & & \\
\hline $\mathrm{H}$ & 0.59204 & 0.47072 & 0.58207 & & & & \\
\hline $\mathrm{H}$ & 0.60534 & 0.53393 & 0.62473 & $\mathrm{C}$ & 0.38029 & 0.56007 & 0.42033 \\
\hline $\mathrm{H}$ & 0.57735 & 0.58268 & 0.51657 & $\mathrm{C}$ & 0.34955 & 0.56799 & 0.39539 \\
\hline $\mathrm{H}$ & 0.60996 & 0.61948 & 0.46593 & $\mathrm{C}$ & 0.33823 & 0.53282 & 0.38004 \\
\hline $\mathrm{H}$ & 0.63552 & 0.68470 & 0.47966 & $\mathrm{C}$ & 0.36249 & 0.50485 & 0.39650 \\
\hline $\mathrm{H}$ & 0.62826 & 0.71194 & 0.54525 & $\mathrm{C}$ & 0.36321 & 0.46132 & 0.39298 \\
\hline $\mathrm{H}$ & 0.59598 & 0.67485 & 0.59582 & $\mathrm{C}$ & 0.34131 & 0.44242 & 0.42684 \\
\hline $\mathrm{H}$ & 0.63634 & 0.59108 & 0.61054 & $\mathrm{C}$ & 0.33523 & 0.46155 & 0.46145 \\
\hline $\mathrm{H}$ & 0.66965 & 0.60041 & 0.67209 & $\mathrm{C}$ & 0.31745 & 0.44302 & 0.49206 \\
\hline $\mathrm{H}$ & 0.63493 & 0.62854 & 0.72780 & $\mathrm{C}$ & 0.30544 & 0.40516 & 0.48852 \\
\hline $\mathrm{H}$ & 0.56751 & 0.65035 & 0.71944 & $\mathrm{C}$ & 0.31127 & 0.38589 & 0.45404 \\
\hline $\mathrm{H}$ & 0.53503 & 0.64216 & 0.65735 & $\mathrm{C}$ & 0.32900 & 0.40440 & 0.42352 \\
\hline $\mathrm{H}$ & 0.52066 & 0.65548 & 0.53960 & $\mathrm{C}$ & 0.34432 & 0.44747 & 0.35562 \\
\hline $\mathrm{H}$ & 0.44447 & 0.66018 & 0.55262 & $\mathrm{C}$ & 0.30561 & 0.45567 & 0.34885 \\
\hline $\mathrm{H}$ & 0.50057 & 0.59486 & 0.67151 & $\mathrm{C}$ & 0.28740 & 0.44301 & 0.31578 \\
\hline $\mathrm{H}$ & 0.46788 & 0.56312 & 0.64909 & $\mathrm{C}$ & 0.30730 & 0.42124 & 0.28883 \\
\hline $\mathrm{H}$ & 0.51815 & 0.55891 & 0.63996 & $\mathrm{C}$ & 0.34538 & 0.41185 & 0.29576 \\
\hline $\mathrm{H}$ & 0.37687 & 0.58962 & 0.55316 & $\mathrm{C}$ & 0.36352 & 0.42466 & 0.32899 \\
\hline $\mathrm{H}$ & 0.33249 & 0.62559 & 0.51220 & $\mathrm{C}$ & 0.40486 & 0.44868 & 0.39669 \\
\hline $\mathrm{H}$ & 0.31702 & 0.69378 & 0.52712 & $\mathrm{C}$ & 0.42100 & 0.42525 & 0.42438 \\
\hline $\mathrm{H}$ & 0.34721 & 0.72468 & 0.58395 & $\mathrm{C}$ & 0.46085 & 0.42340 & 0.41773 \\
\hline $\mathrm{H}$ & 0.39158 & 0.68831 & 0.62501 & $\mathrm{C}$ & 0.46922 & 0.44573 & 0.38594 \\
\hline $\mathrm{H}$ & 0.46990 & 0.65013 & 0.66616 & $\mathrm{C}$ & 0.42993 & 0.48665 & 0.34029 \\
\hline $\mathrm{H}$ & 0.46165 & 0.66770 & 0.73406 & $\mathrm{C}$ & 0.50836 & 0.45424 & 0.36851 \\
\hline $\mathrm{H}$ & 0.39885 & 0.65773 & 0.76636 & $\mathrm{C}$ & 0.53854 & 0.43762 & 0.39677 \\
\hline $\mathrm{H}$ & 0.34400 & 0.63161 & 0.72880 & $\mathrm{C}$ & 0.55576 & 0.46038 & 0.42483 \\
\hline $\mathrm{H}$ & 0.35273 & 0.61374 & 0.66108 & $\mathrm{C}$ & 0.58112 & 0.44448 & 0.45125 \\
\hline $\mathrm{H}$ & 0.37957 & 0.55390 & 0.67091 & $\mathrm{C}$ & 0.58960 & 0.40554 & 0.45012 \\
\hline $\mathrm{H}$ & 0.36433 & 0.48885 & 0.63225 & $\mathrm{C}$ & 0.57274 & 0.38262 & 0.42212 \\
\hline $\mathrm{H}$ & 0.39594 & 0.49830 & 0.56143 & $\mathrm{C}$ & 0.54750 & 0.39854 & 0.39564 \\
\hline $\mathrm{H}$ & 0.37594 & 0.38174 & 0.47755 & $\mathrm{C}$ & 0.51390 & 0.43477 & 0.32942 \\
\hline $\mathrm{H}$ & 0.37029 & 0.31130 & 0.46914 & $\mathrm{C}$ & 0.48517 & 0.41289 & 0.31231 \\
\hline $\mathrm{H}$ & 0.42892 & 0.27180 & 0.45997 & $\mathrm{C}$ & 0.49116 & 0.39459 & 0.27729 \\
\hline $\mathrm{H}$ & 0.49255 & 0.30336 & 0.45443 & $\mathrm{C}$ & 0.52633 & 0.39767 & 0.25883 \\
\hline $\mathrm{H}$ & 0.49789 & 0.37398 & 0.46144 & $\mathrm{C}$ & 0.55556 & 0.41885 & 0.27598 \\
\hline $\mathrm{H}$ & 0.45571 & 0.56323 & 0.48710 & $\mathrm{C}$ & 0.54944 & 0.43700 & 0.31091 \\
\hline $\mathrm{N}$ & 0.38786 & 0.55318 & 0.41477 & $\mathrm{C}$ & 0.51232 & 0.49747 & 0.36574 \\
\hline $\mathrm{N}$ & 0.43022 & 0.49554 & 0.36563 & $\mathrm{C}$ & 0.53004 & 0.52114 & 0.33803 \\
\hline $\mathrm{N}$ & 0.48991 & 0.55146 & 0.39123 & $\mathrm{C}$ & 0.52494 & 0.55877 & 0.35064 \\
\hline $\mathrm{N}$ & 0.54214 & 0.54701 & 0.55434 & $\mathrm{C}$ & 0.50416 & 0.55644 & 0.38521 \\
\hline $\mathrm{N}$ & 0.49053 & 0.60391 & 0.61222 & $\mathrm{C}$ & 0.54063 & 0.46771 & 0.54293 \\
\hline $\mathrm{N}$ & 0.41439 & 0.55256 & 0.58205 & $\mathrm{C}$ & 0.57019 & 0.45462 & 0.56720 \\
\hline $\mathrm{Yb}$ & 0.44590 & 0.52551 & 0.43679 & $\mathrm{C}$ & 0.58376 & 0.48673 & 0.58675 \\
\hline $\mathrm{Yb}$ & 0.47458 & 0.55700 & 0.54764 & $\mathrm{C}$ & 0.56188 & 0.51822 & 0.57349 \\
\hline $\mathrm{O}$ & 0.43279 & 0.43368 & 0.57785 & $\mathrm{C}$ & 0.56351 & 0.56074 & 0.58310 \\
\hline $\mathrm{O}$ & 0.50729 & 0.43556 & 0.55515 & $\mathrm{C}$ & 0.58584 & 0.58330 & 0.55187 \\
\hline $\mathrm{O}$ & 0.47325 & 0.49515 & 0.57981 & $\mathrm{C}$ & 0.58959 & 0.56949 & 0.51451 \\
\hline $\mathrm{Si}$ & 0.46552 & 0.45698 & 0.55233 & $\mathrm{C}$ & 0.60792 & 0.59128 & 0.48651 \\
\hline
\end{tabular}




\begin{tabular}{|c|c|c|c|}
\hline & 0.62284 & & \\
\hline & 0.61924 & & \\
\hline & & & \\
\hline & & & \\
\hline & 62204 & & \\
\hline & 64139 & & \\
\hline & 62289 & & \\
\hline & .58514 & & \\
\hline & .56585 & & \\
\hline & 52256 & & \\
\hline & & & \\
\hline & 766 & & \\
\hline & & & \\
\hline & & & \\
\hline & & & \\
\hline & & & \\
\hline & & & \\
\hline & & & \\
\hline & 036 & & \\
\hline & 701 & & \\
\hline & & & \\
\hline & & & \\
\hline & & & \\
\hline & & & \\
\hline & & & \\
\hline & & & \\
\hline & & & \\
\hline & & & \\
\hline & & & \\
\hline & & & \\
\hline & & & \\
\hline & & & \\
\hline 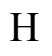 & & & \\
\hline & & & \\
\hline & 0.34489 & & \\
\hline & & & \\
\hline $\mathbf{I}$ & 0.29152 & & \\
\hline & 0.30200 & & \\
\hline & & & \\
\hline & 0.28933 & & \\
\hline $\mathrm{H}$ & 0.25733 & & \\
\hline & 0.29304 & & \\
\hline $\mathrm{r}$ & & & \\
\hline $\mathrm{H}$ & 0.39297 & & \\
\hline $\mathrm{H}$ & 0.40542 & & 473 \\
\hline & & & \\
\hline & & & \\
\hline $\mathrm{H}$ & 0.45510 & 0.50505 & \\
\hline $\mathrm{H}$ & 0.40453 & 0.50455 & 0.3446 \\
\hline
\end{tabular}

$\begin{array}{llll}\mathrm{H} & 0.54898 & 0.49076 & 0.42632\end{array}$

$\begin{array}{llll}\mathrm{H} & 0.59392 & 0.46271 & 0.47310\end{array}$

$\begin{array}{llll}\mathrm{H} & 0.60929 & 0.39317 & 0.47091\end{array}$

$\begin{array}{llll}\mathrm{H} & 0.57921 & 0.35212 & 0.42080\end{array}$

$\begin{array}{llll}\mathrm{H} & 0.53453 & 0.38031 & 0.37391\end{array}$

$\begin{array}{llll}\mathrm{H} & 0.45782 & 0.40924 & 0.32680\end{array}$

$\begin{array}{lllll}\mathrm{H} & 0.46821 & 0.37762 & 0.26468\end{array}$

$\mathrm{H} \quad 0.53115 \quad 0.38326 \quad 0.23159$

$\begin{array}{llll}\mathrm{H} & 0.58350 & 0.42080 & 0.26224\end{array}$

$\begin{array}{lllll}\mathrm{H} & 0.57287 & 0.45244 & 0.32453\end{array}$

$\begin{array}{llll}\mathrm{H} & 0.54416 & 0.51197 & 0.31199\end{array}$

$\begin{array}{llll}\mathrm{H} & 0.53472 & 0.58473 & 0.33664\end{array}$

$\begin{array}{llll}\mathrm{H} & 0.49436 & 0.57941 & 0.40381\end{array}$

$\mathrm{H} \quad 0.52332 \quad 0.45175 \quad 0.52256$

$\begin{array}{llll}\mathrm{H} & 0.58037 & 0.42538 & 0.56977\end{array}$

$\begin{array}{llll}\mathrm{H} & 0.60615 & 0.48729 & 0.60820\end{array}$

$\begin{array}{llll}\mathrm{H} & 0.57773 & 0.54163 & 0.50717\end{array}$

$\begin{array}{llll}\mathrm{H} & 0.61036 & 0.58002 & 0.45751\end{array}$

$\mathrm{H} \quad 0.63715 \quad 0.64418 \quad 0.47356$

$\begin{array}{llll}\mathrm{H} & 0.63079 & 0.66919 & 0.54008\end{array}$

$\begin{array}{llll}\mathrm{H} & 0.59828 & 0.63070 & 0.58953\end{array}$

$\mathrm{H} \quad 0.63712 \quad 0.54438 \quad 0.60156$

$\begin{array}{llll}\mathrm{H} & 0.67122 & 0.55400 & 0.66268\end{array}$

$\begin{array}{llll}\mathrm{H} & 0.63798 & 0.58457 & 0.71792\end{array}$

$\mathrm{H} \quad 0.57050 \quad 0.60637 \quad 0.71036$

$\begin{array}{llll}\mathrm{H} & 0.53669 & 0.59667 & 0.64926\end{array}$

$\begin{array}{llll}\mathrm{H} & 0.52364 & 0.62090 & 0.53823\end{array}$

$\begin{array}{llll}\mathrm{H} & 0.44742 & 0.62532 & 0.55178\end{array}$

$\begin{array}{llll}\mathrm{H} & 0.49978 & 0.54255 & 0.66040\end{array}$

$\begin{array}{llll}\mathrm{H} & 0.46994 & 0.51266 & 0.63168\end{array}$

$\begin{array}{llll}\mathrm{H} & 0.52053 & 0.51234 & 0.62463\end{array}$

$\begin{array}{llll}\mathrm{H} & 0.37885 & 0.54657 & 0.54460\end{array}$

$\begin{array}{llll}\mathrm{H} & 0.33569 & 0.58464 & 0.50368\end{array}$

$\begin{array}{llll}\mathrm{H} & 0.32131 & 0.65263 & 0.52005\end{array}$

$\begin{array}{llll}\mathrm{H} & 0.35102 & 0.68173 & 0.57804\end{array}$

$\begin{array}{lllll}\mathrm{H} & 0.39428 & 0.64359 & 0.61878\end{array}$

$\begin{array}{llll}\mathrm{H} & 0.47089 & 0.60458 & 0.65871\end{array}$

$\begin{array}{lllll}\mathrm{H} & 0.46118 & 0.62367 & 0.72592\end{array}$

$\begin{array}{llll}\mathrm{H} & 0.39793 & 0.61357 & 0.75731\end{array}$

$\mathrm{H} \quad 0.34455 \quad 0.58452 \quad 0.71986$

$\begin{array}{llll}\mathrm{H} & 0.35461 & 0.56537 & 0.65260\end{array}$

$\begin{array}{llll}\mathrm{H} & 0.38122 & 0.50412 & 0.65514\end{array}$

$\begin{array}{llll}\mathrm{H} & 0.38830 & 0.43643 & 0.61832\end{array}$

$\begin{array}{llll}\mathrm{H} & 0.42892 & 0.45205 & 0.55278\end{array}$

$\begin{array}{llll}\mathrm{H} & 0.46429 & 0.48140 & 0.49715\end{array}$

$\begin{array}{llll}\mathrm{H} & 0.46125 & 0.55309 & 0.47297\end{array}$

$\begin{array}{llll}\mathrm{N} & 0.38830 & 0.52193 & 0.42085\end{array}$

$\begin{array}{llll}\mathrm{N} & 0.43470 & 0.46176 & 0.37350\end{array}$

$\begin{array}{llll}\mathrm{N} & 0.49646 & 0.51934 & 0.39435\end{array}$ 
$\begin{array}{cccc}\mathrm{N} & 0.53544 & 0.50610 & 0.54695 \\ \mathrm{~N} & 0.49224 & 0.56045 & 0.60308 \\ \mathrm{~N} & 0.42848 & 0.50952 & 0.57238 \\ \mathrm{Yb} & 0.44928 & 0.49957 & 0.43922 \\ \mathrm{Yb} & 0.47635 & 0.53433 & 0.53128\end{array}$

\section{Int III:}

$\begin{array}{llll}\mathrm{C} & 0.44687 & 0.51318 & 0.62349 \\ \mathrm{C} & 0.44588 & 0.48898 & 0.65551 \\ \mathrm{C} & 0.41858 & 0.49388 & 0.68410 \\ \mathrm{C} & 0.39122 & 0.52286 & 0.68113 \\ \mathrm{C} & 0.39137 & 0.54654 & 0.64910 \\ \mathrm{C} & 0.41890 & 0.54158 & 0.62053 \\ \mathrm{C} & 0.47200 & 0.46426 & 0.57835 \\ \mathrm{C} & 0.50317 & 0.43967 & 0.57334 \\ \mathrm{C} & 0.49755 & 0.40192 & 0.56156 \\ \mathrm{C} & 0.46081 & 0.38831 & 0.55412 \\ \mathrm{C} & 0.42956 & 0.41278 & 0.55854 \\ \mathrm{C} & 0.43516 & 0.45029 & 0.57081 \\ \mathrm{C} & 0.45239 & 0.65368 & 0.53043 \\ \mathrm{C} & 0.42140 & 0.63853 & 0.55083 \\ \mathrm{C} & 0.39504 & 0.66210 & 0.56936 \\ \mathrm{C} & 0.39898 & 0.70170 & 0.56767 \\ \mathrm{C} & 0.42884 & 0.71730 & 0.54622 \\ \mathrm{C} & 0.45486 & 0.69359 & 0.52746 \\ \mathrm{C} & 0.47367 & 0.63100 & 0.46572 \\ \mathrm{C} & 0.43668 & 0.63784 & 0.45188 \\ \mathrm{C} & 0.42962 & 0.64057 & 0.41271 \\ \mathrm{C} & 0.45953 & 0.63663 & 0.38659 \\ \mathrm{C} & 0.49639 & 0.62951 & 0.40008 \\ \mathrm{C} & 0.50343 & 0.62662 & 0.43927 \\ \mathrm{C} & 0.39757 & 0.43158 & 0.43597 \\ \mathrm{C} & 0.39452 & 0.46426 & 0.45909 \\ \mathrm{C} & 0.36878 & 0.46571 & 0.48948 \\ \mathrm{C} & 0.34535 & 0.43439 & 0.49751 \\ \mathrm{C} & 0.34832 & 0.40165 & 0.47496 \\ \mathrm{C} & 0.37414 & 0.40026 & 0.44449 \\ \mathrm{C} & 0.41512 & 0.40160 & 0.37195 \\ \mathrm{C} & 0.39672 & 0.41093 & 0.33769 \\ \mathrm{C} & 0.38756 & 0.38264 & 0.31099 \\ \mathrm{C} & 0.39666 & 0.34443 & 0.31786 \\ \mathrm{C} & 0.41505 & 0.33468 & 0.35192 \\ \mathrm{C} & 0.42407 & 0.36296 & 0.37855 \\ \mathrm{C} & 0.59526 & 0.38404 & 0.43362 \\ \mathrm{C} & 0.58888 & 0.35099 & 0.45551 \\ \mathrm{C} & 0.61395 & 0.34055 & 0.48465 \\ \mathrm{C} & 0.64654 & 0.36250 & 0.49192 \\ \mathrm{C} & 0.65343 & 0.39497 & 0.46990 \\ \mathrm{C} & 0.62779 & 0.40594 & 0.44124\end{array}$

$\begin{array}{llll}\text { C } & 0.56824 & 0.35834 & 0.37398\end{array}$

$\begin{array}{llll}\text { C } & 0.53556 & 0.33653 & 0.36653\end{array}$

$\begin{array}{llll}\text { C } & 0.53739 & 0.30394 & 0.34346\end{array}$

$\begin{array}{llll}\text { C } & 0.57209 & 0.29237 & 0.32766\end{array}$

$\begin{array}{llll}\text { C } & 0.60513 & 0.31338 & 0.33567\end{array}$

$\begin{array}{llll}\text { C } & 0.60322 & 0.34573 & 0.35880\end{array}$

$\begin{array}{llll}\text { C } & 0.58385 & 0.62252 & 0.52400\end{array}$

$\begin{array}{llll}\text { C } & 0.58091 & 0.66235 & 0.52943\end{array}$

$\begin{array}{llll}\text { C } & 0.54201 & 0.67131 & 0.52582\end{array}$

$\begin{array}{llll}\text { C } & 0.52280 & 0.63656 & 0.51815\end{array}$

$\begin{array}{llll}\text { C } & 0.48099 & 0.62737 & 0.50946\end{array}$

$\begin{array}{llll}\text { C } & 0.49221 & 0.59421 & 0.59010\end{array}$

$\begin{array}{llll}\text { C } & 0.47392 & 0.58590 & 0.52055\end{array}$

$\begin{array}{llll}\text { C } & 0.46101 & 0.55586 & 0.49792\end{array}$

$\begin{array}{llll}\text { C } & 0.45957 & 0.52263 & 0.52094\end{array}$

$\begin{array}{llll}\text { C } & 0.47141 & 0.53228 & 0.55772\end{array}$

$\begin{array}{llll}\text { C } & 0.47707 & 0.50619 & 0.59214\end{array}$

$\begin{array}{llll}\text { C } & 0.51727 & 0.51280 & 0.60733\end{array}$

$\begin{array}{llll}\text { C } & 0.53122 & 0.51469 & 0.64513\end{array}$

$\begin{array}{llll}\text { C } & 0.57041 & 0.52276 & 0.64228\end{array}$

$\begin{array}{llll}\text { C } & 0.57851 & 0.52554 & 0.60287\end{array}$

$\begin{array}{llll}\text { C } & 0.61724 & 0.54030 & 0.46592\end{array}$

$\begin{array}{llll}\text { C } & 0.65770 & 0.52305 & 0.46459\end{array}$

$\begin{array}{llll}\text { C } & 0.66020 & 0.49851 & 0.50110\end{array}$

$\begin{array}{llll}\text { C } & 0.61848 & 0.49114 & 0.51049\end{array}$

$\begin{array}{llll}\text { C } & 0.58663 & 0.49171 & 0.37024\end{array}$

$\begin{array}{llll}\text { C } & 0.60726 & 0.47427 & 0.34057\end{array}$

$\begin{array}{llll}\text { C } & 0.60366 & 0.43460 & 0.34618\end{array}$

$\begin{array}{llll}\text { C } & 0.58109 & 0.42974 & 0.37921\end{array}$

$\begin{array}{llll}\text { C } & 0.56791 & 0.39383 & 0.40017\end{array}$

$\begin{array}{llll}\text { C } & 0.49773 & 0.41932 & 0.35268\end{array}$

$\begin{array}{llll}\text { C } & 0.52836 & 0.40193 & 0.41647\end{array}$

$\begin{array}{llll}\text { C } & 0.51598 & 0.40173 & 0.45424\end{array}$

$\begin{array}{llll}\text { C } & 0.47694 & 0.41273 & 0.45496\end{array}$

$\begin{array}{llll}\text { C } & 0.46559 & 0.41995 & 0.41768\end{array}$

$\begin{array}{llll}\text { C } & 0.42664 & 0.43162 & 0.40224\end{array}$

$\begin{array}{llll}\text { C } & 0.42913 & 0.47261 & 0.38772\end{array}$

$\begin{array}{llll}\text { C } & 0.40006 & 0.49639 & 0.37227\end{array}$

$\begin{array}{llll}\text { C } & 0.41549 & 0.53329 & 0.37007\end{array}$

$\begin{array}{llll}\mathrm{C} & 0.45305 & 0.53061 & 0.38421\end{array}$

$\begin{array}{llll}\mathrm{H} & 0.46644 & 0.46555 & 0.65761\end{array}$

$\begin{array}{llll}\mathrm{H} & 0.41844 & 0.47465 & 0.70873\end{array}$

$\begin{array}{llll}\mathrm{H} & 0.36971 & 0.52662 & 0.70348\end{array}$

$\begin{array}{llll}\mathrm{H} & 0.36985 & 0.56901 & 0.64600\end{array}$

$\begin{array}{llll}\mathrm{H} & 0.41799 & 0.55991 & 0.59529\end{array}$

$\begin{array}{llll}\mathrm{H} & 0.53206 & 0.45010 & 0.57869\end{array}$

$\begin{array}{llll}\mathrm{H} & 0.52226 & 0.38307 & 0.55818\end{array}$

$\begin{array}{llll}\mathrm{H} & 0.45648 & 0.35882 & 0.54474\end{array}$

$\begin{array}{llll}\mathrm{H} & 0.40066 & 0.40277 & 0.55219\end{array}$ 


$\begin{array}{llll}\mathrm{H} & 0.41044 & 0.46903 & 0.57462 \\ \mathrm{H} & 0.41722 & 0.60760 & 0.55176 \\ \mathrm{H} & 0.37127 & 0.64922 & 0.58506 \\ \mathrm{H} & 0.37871 & 0.72031 & 0.58245 \\ \mathrm{H} & 0.43185 & 0.74832 & 0.54384 \\ \mathrm{H} & 0.47688 & 0.70646 & 0.50954 \\ \mathrm{H} & 0.41296 & 0.64124 & 0.47192 \\ \mathrm{H} & 0.40051 & 0.64587 & 0.40264 \\ \mathrm{H} & 0.45409 & 0.63888 & 0.35590 \\ \mathrm{H} & 0.52007 & 0.62611 & 0.37995 \\ \mathrm{H} & 0.53234 & 0.62073 & 0.44939 \\ \mathrm{H} & 0.41215 & 0.48926 & 0.45276 \\ \mathrm{H} & 0.36697 & 0.49166 & 0.50681 \\ \mathrm{H} & 0.32488 & 0.43554 & 0.52110 \\ \mathrm{H} & 0.33023 & 0.37684 & 0.48082 \\ \mathrm{H} & 0.37545 & 0.37445 & 0.42703 \\ \mathrm{H} & 0.39032 & 0.44073 & 0.33117 \\ \mathrm{H} & 0.37341 & 0.39077 & 0.28433 \\ \mathrm{H} & 0.38969 & 0.32243 & 0.29680 \\ \mathrm{H} & 0.42264 & 0.30494 & 0.35785 \\ \mathrm{H} & 0.43882 & 0.35495 & 0.40495 \\ \mathrm{H} & 0.56408 & 0.33300 & 0.44930 \\ \mathrm{H} & 0.60829 & 0.31480 & 0.50145 \\ \mathrm{H} & 0.66659 & 0.35404 & 0.51435 \\ \mathrm{H} & 0.67926 & 0.41187 & 0.47477 \\ \mathrm{H} & 0.63342 & 0.43144 & 0.42410 \\ \mathrm{H} & 0.50812 & 0.34456 & 0.37905 \\ \mathrm{H} & 0.51132 & 0.28756 & 0.33800 \\ \mathrm{H} & 0.57354 & 0.26691 & 0.30961 \\ \mathrm{H} & 0.63273 & 0.30425 & 0.32418 \\ \mathrm{H} & 0.62945 & 0.36111 & 0.36594 \\ \mathrm{H} & 0.60939 & 0.60464 & 0.52511 \\ \mathrm{H} & 0.60429 & 0.68217 & 0.53484 \\ \mathrm{H} & 0.52974 & 0.69964 & 0.52857 \\ \mathrm{H} & 0.46699 & 0.60656 & 0.60424 \\ \mathrm{H} & 0.50782 & 0.57613 & 0.61045\end{array}$

$\begin{array}{lccc}\mathrm{H} & 0.51104 & 0.61739 & 0.58035 \\ \mathrm{H} & 0.45413 & 0.55791 & 0.46773 \\ \mathrm{H} & 0.45154 & 0.49409 & 0.51189 \\ \mathrm{H} & 0.51475 & 0.51149 & 0.67127 \\ \mathrm{H} & 0.59062 & 0.52613 & 0.66569 \\ \mathrm{H} & 0.60585 & 0.53185 & 0.58925 \\ \mathrm{H} & 0.59925 & 0.52992 & 0.44217 \\ \mathrm{H} & 0.61641 & 0.57172 & 0.46638 \\ \mathrm{H} & 0.67995 & 0.54524 & 0.46390 \\ \mathrm{H} & 0.66098 & 0.50538 & 0.43876 \\ \mathrm{H} & 0.67642 & 0.47195 & 0.49687 \\ \mathrm{H} & 0.67370 & 0.51460 & 0.52458 \\ \mathrm{H} & 0.61230 & 0.48468 & 0.54063 \\ \mathrm{H} & 0.60603 & 0.46847 & 0.49225 \\ \mathrm{H} & 0.58202 & 0.52214 & 0.37540 \\ \mathrm{H} & 0.62262 & 0.48864 & 0.31764 \\ \mathrm{H} & 0.61551 & 0.41226 & 0.32816 \\ \mathrm{H} & 0.48036 & 0.44444 & 0.34551 \\ \mathrm{H} & 0.52727 & 0.42432 & 0.34334 \\ \mathrm{H} & 0.48621 & 0.39398 & 0.33812 \\ \mathrm{H} & 0.53346 & 0.39501 & 0.47896 \\ \mathrm{H} & 0.45901 & 0.41527 & 0.48017 \\ \mathrm{H} & 0.37090 & 0.48794 & 0.36546 \\ \mathrm{H} & 0.40153 & 0.55903 & 0.35954 \\ \mathrm{H} & 0.47442 & 0.55317 & 0.38688 \\ \mathrm{H} & 0.52679 & 0.48256 & 0.49211 \\ \mathrm{H} & 0.52875 & 0.53398 & 0.45322 \\ \mathrm{~N} & 0.54897 & 0.60680 & 0.51682 \\ \mathrm{~N} & 0.48069 & 0.57126 & 0.55714 \\ \mathrm{~N} & 0.54655 & 0.51940 & 0.58144 \\ \mathrm{~N} & 0.57062 & 0.46486 & 0.39389 \\ \mathrm{~N} & 0.49737 & 0.41398 & 0.39416 \\ \mathrm{~N} & 0.46128 & 0.49385 & 0.39536 \\ \mathrm{Yb} & 0.53695 & 0.54011 & 0.51705 \\ \mathrm{Yb} & 0.51602 & 0.47645 & 0.43111 \\ \mathrm{O} & 0.60042 & 0.52736 & 0.50169\end{array}$




\section{References:}

(1) Yamaguchi, K.; Wang, Y.; Oishi, T.; Kuroda, Y.; Mizuno, N. Heterogeneously Catalyzed Aerobic Cross-Dehydrogenative Coupling of Terminal Alkynes and Monohydrosilanes by Gold Supported on OMS-2. Angew. Chem. Int. Ed. 2013, 52, 5627-5630.

(2) Yu, B.; Yang, P.; Gao, X.; Yang, Z.; Zhao, Y.; Zhang, H.; Liu, Z. Sequential protocol for $\mathrm{C}(\mathrm{sp})-\mathrm{H}$ carboxylation with $\mathrm{CO}_{2}: \mathrm{KO}^{t} \mathrm{Bu}$-catalyzed $\mathrm{C}(\mathrm{sp})-\mathrm{H}$ silylation and $\mathrm{KO}^{t} \mathrm{Bu}-$ mediated carboxylation. Sci. China Chem. 2018, $61,449-456$.

(3) Singh, C.; Prakasham, A. P.; Kumar Gangwar, M.; J.Butcher, R.; Ghosh, P. One-Pot Tandem Hiyama Alkynylation/Cyclizations by Palladium(II) Acyclic Diaminocarbene (ADC) Complexes Yielding Biologically Relevant Benzofuran Scaffolds. ACS Omega. 2018, 3, 1740-1756.

(4) Soler, J. M.; Artacho, E.; Gale, J. D.; Garcia, A.; Junquera, J.; Ordejon, P.; Sanchez-Portal, D. The SIESTA method for ab initio order- $N$ materials simulation. J. Phys.: Condens. Matter. 2002, 14, 2745-2779.

(5) Junquera, J.; Paz, O.; Sanchez-Portal, D.; Artacho, E. Numerical atomic orbitals for linearscaling calculations. Phys. Rev. B: Condens. Matter. 2001, 64, 235111.

(6) Troullier, N.; Martins, J. L. Efficient Pseudopotentials for Plane-wave Calculations. Phys. Rev. B: Condens. Matter. 1991, 43, 1993-1996.

(7) Perdew, J. P.; Burke, K.; Ernzerhof, M. Generalized Gradient Approximation Made Simple. Phys. Rev. Lett. 1996, 77, 3865-3868 .

(8) Dion, M.; Rydberg, H.; Schroder, E.; Langreth, D. C.; Lundqvist, B. I. Van der Waals Density Functional for General Geometries. Phys. Rev. Lett. 2004, 92, 246401.

(9) Alavi, A.; Hu, P. J.; Deutsch, T.; Silvestrelli, P. L.; Hutter, J. CO Oxidation on Pt(111): An Ab Initio Density Functional Theory Study. Phys. Rev. Lett. 1998, 80, 3650-3653. 